\title{
THE PALOMAR/KECK ADAPTIVE OPTICS SURVEY OF YOUNG SOLAR ANALOGS: EVIDENCE FOR A UNIVERSAL COMPANION MASS FUNCTION
}

\author{
Stanimir A. Metchev ${ }^{1,3}$ and Lynne A. Hillenbrand ${ }^{2}$ \\ ${ }^{1}$ Department of Physics and Astronomy, 430 Portola Plaza, University of California, Los Angeles, CA 90095-1547, USA; metchev@astro.ucla.edu \\ 2 Department of Physics, Mathematics and Astronomy, MC 105-24, California Institute of Technology, Pasadena, CA 91125, USA \\ Received 2008 January 25; accepted 2008 September 25; published 2009 February 20
}

\begin{abstract}
We present results from an adaptive optics survey for substellar and stellar companions to Sun-like stars. The survey targeted 266 F5-K5 stars in the 3 Myr-3 Gyr age range with distances of 10-190 pc. Results from the survey include the discovery of two brown dwarf companions (HD 49197B and HD 203030B), 24 new stellar binaries, and a triple system. We infer that the frequency of $0.012-0.072 M_{\odot}$ brown dwarfs in $28-1590 \mathrm{AU}$ orbits around young solar analogs is $3.2_{-2.7}^{+3.1} \%$ ( $2 \sigma$ limits). The result demonstrates that the deficiency of substellar companions at wide orbital separations from Sun-like stars is less pronounced than in the radial velocity "brown dwarf desert." We infer that the mass distribution of companions in 28-1590 AU orbits around solar-mass stars follows a continuous $d N / d M_{2} \propto M_{2}^{-0.4}$ relation over the $0.01-1.0 M_{\odot}$ secondary mass range. While this functional form is similar to that for isolated objects less than $0.1 M_{\odot}$, over the entire $0.01-1.0 M_{\odot}$ range, the mass functions of companions and of isolated objects differ significantly. Based on this conclusion and on similar results from other direct imaging and radial velocity companion surveys in the literature, we argue that the companion mass function follows the same universal form over the entire range between 0 and $1590 \mathrm{AU}$ in orbital semimajor axis and $\approx 0.01-20 M_{\odot}$ in companion mass. In this context, the relative dearth of substellar versus stellar secondaries at all orbital separations arises naturally from the inferred form of the companion mass function.
\end{abstract}

Key words: binaries: visual - stars: imaging - stars: low-mass, brown dwarfs - stars: luminosity function, mass function

Online-only material: machine-readable tables

\section{INTRODUCTION}

The properties of brown dwarf companions to stars are important for understanding the substellar companion mass function (CMF), the formation of brown dwarfs, and the formation and evolution of low mass ratio binary systems. Widely separated brown dwarf companions, in particular, are an important benchmark for studying the properties of substellar objects because of their accessibility to direct spectroscopic characterization and their relative ease of age-dating-from assumed coevality with their host stars.

However, brown dwarf companions have been an elusive target for direct imaging. The main challenge has been the need to attain sufficient imaging contrast to detect secondaries that are $>10^{3}$ fainter than their host stars at angular separations spanning solar-system-like scales ( $<40 \mathrm{AU}=00^{\prime} 44$ at $\left.100 \mathrm{pc}\right)$.

The problem is alleviated at young ages when brown dwarfs are brighter. In addition, nearby stars offer an additional advantage because the relevant angular scales are correspondingly wider and more accessible to direct imaging. Young nearby stars are thus the preferred targets for substellar companion searches through direct imaging.

Nevertheless, early surveys for substellar companions, performed with seeing-limited or first-generation high-contrast imaging technology (Oppenheimer et al. 2001; Hinz et al. 2002; McCarthy \& Zuckerman 2004), had very low detection rates, $\lesssim 0.5 \%$. This low brown dwarf companion detection rate was similar to that inferred from precision

\footnotetext{
3 Author's Current address: Department of Physics and Astronomy, State University of New York, Stony Brook, NY 11794-3800, USA; metchev@astro.sunysb.edu.
}

radial velocity surveys $(<0.5 \%$ over $0-3 \mathrm{AU}$; Marcy \& Butler (2000), and prompted McCarthy \& Zuckerman (2004) to conclude that the so-called "brown dwarf desert" extends far beyond the orbital separations probed by radial velocity surveys, out to at least $\approx 1200 \mathrm{AU}$.

Over the past few years, advances in adaptive optics (AO) technology and high-contrast imaging methods have improved the chances for the direct imaging of substellar secondaries. Modern AO systems, with $>200$ corrective elements spread across the beam of a 5-10 m telescope, are able to deliver high order rectification $(<250 \mathrm{~nm}$ root mean square $(\mathrm{rms})$ residual error) of wavefronts perturbed by Earth's turbulent atmosphere at up to kilohertz rates. In addition, our empirical appreciation of the local young stellar population has improved over the past decade, as demonstrated by the recent discoveries of a large number of young $(<500 \mathrm{Myr})$ stellar associations within $200 \mathrm{pc}$ from the Sun (e.g., Kastner et al. 1997; Mamajek et al. 1999; Zuckerman \& Webb 2000; Zuckerman et al. 2001; Montes et al. 2001; Zuckerman \& Song 2004, and references therein). These have allowed us to select more suitable targets for direct imaging searches for substellar companions.

Several recent direct imaging surveys of nearby young stellar associations conducted on high-order AO-equipped telescopes (Neuhäuser \& Guenther 2004, 25 A-M stars; Chauvin et al. 2005a, 2005b, 50 A-M stars) or with the Hubble Space Telescope (HST; Lowrance et al. 2005, 45 A-M stars) have enjoyed higher detection rates $(2 \%-4 \%)$ than the first generation of surveys. In addition, at very wide (>1000 AU) separations, where the detection of brown dwarf companions to solar-neighborhood stars is not hindered by contrast, Gizis et al. (2001) found that the frequency of substellar companions to $\mathrm{F}-\mathrm{K}$ dwarfs is fully consistent with that of 
stellar companions to G dwarfs (Duquennoy \& Mayor 1991). Thus, while the radial velocity "brown dwarf desert" remains nearly void within $3 \mathrm{AU}$ even after the discovery of numerous extrasolar planets over the past decade, brown dwarf secondaries at $>100-1000 \mathrm{AU}$ separations seem to not be as rare.

The precise frequency of substellar companions in direct imaging surveys remains controversial. Several highly sensitive surveys performed with the $H S T$ (Schroeder et al. 2000, $23 \mathrm{~A}-$ M stars; Brandner et al. 2000, 28 G-M stars; Luhman et al. 2005, 150 B-M stars) and with high-order AO (Masciadri et al. 2005, 28 G-M stars; Biller et al. 2007, 54 A-M stars) have reported null detections of substellar companions. Given the low (few percent) detection rate of substellar companions in direct imaging surveys, it is now clear that, with $<50$ targets per sample, some of these surveys were too small to expect to detect even a single brown dwarf companion. However, the nondetection of substellar secondaries in two of the largest surveys (Luhman et al. 2005; Biller et al. 2007) is potentially significant.

Given the current understanding of the importance of stellar mass for (1) stellar multiplicity rates (see review in Sterzik \& Durisen 2004) and (2) binary mass ratio distributions (see review in Burgasser et al. 2007), it is imperative that any study of the substellar companion frequency is considered in the context of the mass distribution of primary stars in the sample. A large survey sample comprising primaries with identical masses would be ideal.

The problem of the brown dwarf companion frequency is perhaps most comprehensively dealt with in the context of solar mass primaries. For these, a uniquely large body of stellar and substellar multiplicity data exists on all orbital scales. On one hand, the exhaustive spectroscopic and imaging study of G dwarf multiples by Duquennoy \& Mayor (1991) provides an important anchor to the properties of $0.1-1.0 M_{\odot}$ stellar companions to Sun-like stars. On the other hand, the results from more than a decade of precision radial velocity surveys for planets around $G$ and $\mathrm{K}$ stars allow a comparison with the planetary-mass end of the substellar companion mass range.

A large uniform sample of young Sun-like stars has been compiled by the Formation and Evolution of Planetary Systems (FEPS) Spitzer Legacy team. The purpose of the FEPS Legacy campaign with Spitzer was to study circumstellar disk evolution in the mid-infrared (mid-IR). However, the sample is also well suited for a high-contrast imaging survey for substellar companions. Seventy percent of the FEPS stars are younger than $\sim 500 \mathrm{Myr}$, and all are within $200 \mathrm{pc}$.

As an auxiliary component to the FEPS program, we imaged most of the northern FEPS sample with the high-order AO systems on the Palomar $5 \mathrm{~m}$ and the Keck $10 \mathrm{~m}$ telescopes. We further expanded our AO survey by observing several dozen additional nearby and mostly young solar analogs. Preliminary results from the project were published in Metchev \& Hillenbrand $(2004,2006)$, including the discoveries of two brown dwarf companions: HD 49197B and HD 203030B. The survey has now been completed, and no further brown dwarf companions have been found. The results were analyzed in Metchev (2006). Here, we present the AO survey in its entirety and focus on the statistical interpretation of the data.

The paper is organized as follows. A full description of the survey sample is given in Section 2. The Palomar and Keck AO observing campaigns and the data reduction and calibration techniques are described in Section 3. The candidate companion detection approach and the survey detection limits are discussed in Section 4. The various methods used for bona fide companion confirmation are presented in Section 5. Section 6 summarizes the results from our survey, including all of the newly-discovered and confirmed substellar and stellar secondaries. Section 7 contains a brief discussion of the various sources of incompleteness and a full discussion of the biases in the survey (a full-fledged incompleteness analysis is presented in the Appendix). In Section 8, we estimate the frequency of wide substellar companions to young solar analogs, and present evidence for trends in the companion mass and companion frequency with semimajor axis and primary mass. In Section 9, we consider the results of the current investigation in the broader context of stellar multiplicity, and suggest the existence of a universal CMF. Section 10 summarizes the findings from our study.

\section{TARGET SAMPLE}

The main criteria used for selecting stars for the survey were Sun-like mass, youth, proximity, and visibility from the northern hemisphere. In this section, we describe how they were applied to generate our AO survey sample.

\subsection{Selection}

The selection of the AO survey sample was largely based on the target selection criteria employed in the construction of the FEPS program sample (Meyer et al. 2006). The FEPS selection criteria will not be reproduced here. The final FEPS target list comprises 328 F5-K5 stars within 200 pc distributed uniformly in logarithmic age intervals between $3 \mathrm{Myr}$ and $3 \mathrm{Gyr}$. Approximately a third of these are members of open clusters and stellar associations, and the remainder are field stars. We observed 228 of the 240 FEPS stars north of $\delta=-30^{\circ}$ with AO at Palomar or Keck.

Further 38 solar analogs were added to the AO survey toward the end of the first epoch of observations to bolster the sample size, mirroring FEPS target selection policy. The additional stars were selected from three sources: (1) the broader compilation of FEPS candidate targets, including stars that had been eliminated from the final FEPS sample based on IR background or age redundancy considerations; (2) the compilations of nearby young stars by Montes et al. (2001) and Wichmann et al. (2003); and (3) our own Palomar echelle survey of nearby stars (White et al. 2007). The final set of 266 targets in our AO sample has spectral type and age distributions similar to those of the FEPS sample.

\subsubsection{Deep and Shallow Samples}

To optimize sensitivity to substellar companions, we chose to observe a portion of the youngest and nearest $\mathrm{AO}$ sample stars with deep coronagraphic exposures. We applied the following additional guidelines to select stars for the deep coronagraphic subsample:

1. stellar activity and lithium levels indicating ages less than $500 \mathrm{Myr}$;

2. no $\Delta K_{S}<4$ objects between 0.8 and $13^{\prime \prime} .0$, as determined from real-time flux ratio measurements during survey observations;

3. nearby stars were given priority over more distant stars;

4. isolated stars, not belonging to one of the young open clusters or stellar associations, were given priority for deep observations. 
The first criterion was motivated by the fact that substellar companions should be intrinsically brightest at the youngest ages. The second constraint was aimed at avoiding the loss of sensitivity to faint objects over a large portion of the detector field of view (FOV) because of the presence of a bright neighboring star. ${ }^{4}$ Binaries with separations $\leqslant 00^{\prime} .8$ had both their components sufficiently well covered by the $1^{\prime \prime}$ coronagraphs in the Palomar and Keck AO cameras that they were allowed in the deep sample. The motivation for the third constraint was to optimize sensitivity to substellar companions at the smallest physical separations. The last criterion was applied to avoid duplication with previous sensitive high angular resolution studies of open clusters: Bouvier et al. (1997, the Pleiades, using AO imaging), Köhler et al. (2000, Upper Scorpius, using speckle interferometry), and Patience et al. (2002, $\alpha$ Persei, using speckle interferometry).

Based on the additional criteria outlined above, 84 of the 228 stars selected from the final FEPS sample and 16 of the 38 additional targets were included in our deep sample. The deep subsample thus consists of 100 F5-K5 stars with ages less than 500 Myr.

All remaining stars were primarily observed in short sequences of noncoronagraphic images to establish stellar multiplicity. These will be referred to as the "shallow" sample. The shallow sample includes 11 stars older than 500 Myr that were also observed with long coronagraphic exposures: 2 Hyades ( 200 Myr) members and nine other stars whose subsequent age dating showed that they were older than originally estimated. Although these 11 stars were observed coronagraphically, for the purpose of limiting our deep sample only to the observations with the highest sensitivity to substellar mass, they are not considered as part of the deep sample.

The deep and shallow sample stars and their characteristics are listed in Tables 1 and 2, respectively. Median age, distance, and spectral-type statistics for the deep, shallow, and complete (deep+shallow) samples are given in Table 3.

\subsection{Stellar Properties}

Our sample stars are near-solar (G2 V) analogs, ranging in spectral type between F5 and $\mathrm{K} 5\left(6300 \mathrm{~K}>T_{\text {eff }}>4400 \mathrm{~K}\right)$ and, depending on stellar age, between IV and $\mathrm{V}$ in luminosity class $(3.4<\log g \leqslant 4.5$ in cgs units). The corresponding mass range, based on dynamical mass estimates in binary systems and on stellar thermodynamic models (Baraffe et al. 1998; D'Antona \& Mazzitelli 1994), is approximately $0.7-1.3 M_{\odot}$, following the design of the FEPS sample (Meyer et al. 2006). For greater detail in the assignment of spectral types, effective temperatures, and surface gravities to FEPS sample stars, we refer the reader to Carpenter et al. (2008). Histograms of the distribution of stellar effective temperatures and masses of all stars in our AO survey sample are shown in Figure 1.

Seventy-nine of our sample stars are members of known young stellar associations: Upper Scorpius, $\alpha$ Persei, the Pleiades, and the Hyades. For these, we have adopted ensemble ages from the literature: 5 Myr for the Upper Scorpius OB association (Preibisch et al. 2002), 80 Myr for $\alpha$ Persei (Ventura et al. 1998), 120 Myr for the Pleiades (Ventura et al. 1998), and 600 Myr for the Hyades (Perryman et al. 1998). Sample stars

\footnotetext{
4 Following more accurate postreduction photometry, a 3". 1 companion to one of the stars in our deep sample, HD 31950, was found to be only

$\Delta K_{S}=3.70$ mag fainter. Although this companion violates criterion 2, we have chosen to keep HD 31950 as a member of the deep sample.
}

that do not belong to any known associations were age-dated following one of two approaches: (1) based on the strength of the chromospheric Ca II H and K (3968 $\AA$ and $3933 \AA$ ) line emission for $>30$ Myr old stars, and using the recent activityage relation of Mamajek \& Hillenbrand (2008); or (2) isochrone fitting for $<30$ Myr old premain-sequence stars using the tracks of Baraffe et al. (1998). Where data from high-dispersion optical spectra were previously available (Strassmeier et al. 2000; White et al. 2007), these were also reviewed for the strength of the lithium $6708 \AA$ absorption line to put additional constraints on the ages. All chromospheric and isochronal ages were assumed uncertain to within a factor of 2 . Ages for a remaining set of 20 stars not present in the extended FEPS sample were taken from the literature (Barrado y Navascues et al. 1997; Gutiérrez et al. 1999; Montes et al. 2001; Wichmann et al. 2003; Nordström et al. 2004). Histograms of the age distribution of the complete survey sample and of the deep subsample are shown in Figure 2.

Distances to 166 sample stars with individual Hipparcos parallaxes were taken from the Hipparcos catalog (Perryman et al. 1997). For further 55 known members of young open clusters and $\mathrm{OB}$ associations, we adopted the corresponding mean cluster distance, calculated from a combination of trigonometric, orbital, secular, and cluster parallaxes in the literature, as inferred from Hipparcos and Tycho-2 (Høg et al. 2000) astrometry, longbaseline interferometry, or high-resolution spectroscopy. The adopted distances for open cluster members were $133 \pm 6$ pc for stars in the Pleiades (a weighted mean of the distances to seven members presented in Pan et al. 2004; Munari et al. 2004; Zwahlen et al. 2004; Southworth et al. 2005; and assuming $\sim 1^{\circ}$ cluster angular extent from Adams et al. 2001) and $190 \pm 11$ pc for stars in $\alpha$ Persei (van Leeuwen 1999, assuming $1^{\circ}$ cluster radius). For stars belonging to the Upper Scorpius association, we adopted $145 \pm 40$ pc (de Zeeuw et al. 1999; Mamajek et al. 2002). All of these distances agree with estimates from mainsequence fitting for the corresponding clusters. For 18 more stars, we adopted secular parallaxes from Mamajek et al. (2002) and Mamajek (2004). Finally, for 27 remaining >30 Myr old stars, we obtained approximate distances based on a combination of moving group association, secular parallax (E. E. Mamajek 2007, private communication), and spectroscopic parallax, with care to avoid redundancy in distance and age derivation. The errors on the distances in these cases were conservatively assumed to be $25 \%-50 \%$. More refined distance and age estimates for these stars will be included in a future publication from the FEPS program.

Accurate proper motions for the sample stars are essential in identifying bona fide companions through multiepoch astrometry. Proper motions for the 166 stars with individual Hipparcos parallaxes were taken from the Hipparcos database. For the remaining 100 stars, proper motions were adopted from The Second U.S. Naval Observatory CCD Astrograph Catalog (UCAC2; Zacharias et al. 2004) and from the Tycho-2 Catalog (Høg et al. 2000). The three catalogs provided similar astrometric accuracy

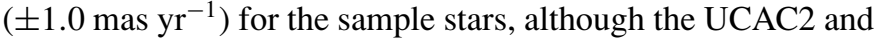
Tycho-2 catalogs went deeper.

Figure 3 presents histograms of the heliocentric distances (Figure (3a)) and total proper motions $\left(\sqrt{\left(\mu_{\alpha} \cos \delta\right)^{2}+\mu_{\delta}^{2}}\right.$; Figure (3b)) of the stars in the complete sample and in the deep subsample. The bimodal distribution of the distances is a combined effect of the large heliocentric distances (130-190 pc) of the youngest (3-120 Myr) stars in the sample and of the preference given to closer systems at older ages. 
Table 1

Deep Sample

\begin{tabular}{|c|c|c|c|c|c|c|c|c|c|c|}
\hline Star & $\begin{array}{c}\alpha \\
(\mathrm{J} 2000.0) \\
\end{array}$ & $\begin{array}{c}\delta \\
(\mathrm{J} 2000.0) \\
\end{array}$ & $\begin{array}{c}\mu_{\alpha} \cos \delta \\
\left(\mathrm{mas}^{-1} \mathrm{yr}^{-1}\right)\end{array}$ & $\begin{array}{c}\mu_{\delta} \\
\left.\left(\operatorname{mas}^{-1}\right)^{-1}\right) \\
\end{array}$ & $\begin{array}{c}d \\
(\mathrm{pc})\end{array}$ & $\begin{array}{c}K_{S} \\
(\mathrm{mag})\end{array}$ & Sp.T. & Association & $\log {\mathrm{Age} \mathrm{yr}^{-1}}^{-1}$ & $\begin{array}{l}\text { Mass } \\
\left(M_{\odot}\right)\end{array}$ \\
\hline HD 377 & $00: 08: 25.74$ & $+06: 37: 00.50$ & $85.2 \pm 1.5$ & $-2.6 \pm 1.4$ & $40.0 \pm 2.0$ & 6.1 & $\mathrm{G} 2 \mathrm{~V}$ & $\ldots$ & 7.6 & 1.1 \\
\hline HD 691 & $00: 11: 22.44$ & $+30: 26: 58.52$ & $209.7 \pm 1.0$ & $35.5 \pm 1.0$ & $34.0 \pm 1.0$ & 6.2 & $\mathrm{~K} 0 \mathrm{~V}$ & $\ldots$ & 8.5 & 1.0 \\
\hline HD 984 & $00: 14: 10.25$ & $-07: 11: 56.92$ & $104.9 \pm 1.3$ & $-67.6 \pm 1.2$ & $46.0 \pm 2.0$ & 6.1 & F7V & $\ldots$ & 7.6 & 1.2 \\
\hline HD 1405 & $00: 18: 20.78$ & $+30: 57: 23.76$ & $141.5 \pm 2.2$ & $-177.0 \pm 2.1$ & $29.0 \pm 10.0$ & 6.4 & $\mathrm{~K} 2 \mathrm{~V}$ & $\ldots$ & 8.0 & 0.8 \\
\hline QT And & $00: 41: 17.32$ & $+34: 25: 16.77$ & $44.8 \pm 0.7$ & $-36.2 \pm 0.8$ & $50.0 \pm 25.0$ & 7.4 & K4 & $\ldots$ & 7.8 & 0.8 \\
\hline HD 7661 & $01: 16: 24.19$ & $-12: 05: 49.33$ & $134.8 \pm 1.1$ & $-5.7 \pm 1.1$ & $27.0 \pm 1.0$ & 5.7 & K0V & $\ldots$ & 8.6 & 1.0 \\
\hline HIP 6276 & $01: 20: 32.27$ & $-11: 28: 03.74$ & $116.0 \pm 1.1$ & $-140.2 \pm 1.1$ & $35.0 \pm 1.0$ & 6.5 & G0 & $\ldots$ & 8.5 & 0.9 \\
\hline HD 8907 & $01: 28: 34.35$ & $+42: 16: 03.70$ & $51.7 \pm 1.0$ & $-99.2 \pm 1.1$ & $34.0 \pm 1.0$ & 5.4 & F8 & $\ldots$ & 8.3 & 1.2 \\
\hline HD 12039 & $01: 57: 48.98$ & $-21: 54: 05.32$ & $102.4 \pm 1.2$ & $-48.0 \pm 1.1$ & $42.0 \pm 2.0$ & 6.5 & $\mathrm{G} 3 / 5 \mathrm{~V}$ & $\ldots$ & 7.5 & 1.0 \\
\hline HD 15526 & $02: 29: 35.03$ & $-12: 24: 08.56$ & $42.1 \pm 1.3$ & $-12.2 \pm 1.1$ & $106.0 \pm 26.0$ & 8.0 & $\mathrm{G} 5 / 6 \mathrm{~V}$ & $\ldots$ & 7.6 & 0.9 \\
\hline 1RXS J025216.9+361658 & $02: 52: 17.59$ & $+36: 16: 48.14$ & $53.4 \pm 1.3$ & $-40.1 \pm 0.7$ & $100.0 \pm 50.0$ & 7.6 & K2IV & $\ldots$ & 7.8 & 1.1 \\
\hline HD 17925 & $02: 52: 32.14$ & $-12: 46: 11.18$ & $397.3 \pm 1.2$ & $-189.9 \pm 1.3$ & $10.0 \pm 0.1$ & 4.1 & K1V & $\ldots$ & 7.9 & 0.9 \\
\hline 1RXS J025751.8+115759 & $02: 57: 51.68$ & $+11: 58: 05.83$ & $31.4 \pm 1.2$ & $-28.4 \pm 1.2$ & $118.0 \pm 16.0$ & 8.5 & G7V & $\ldots$ & 7.8 & 1.1 \\
\hline RX J0258.4+2947 & $02: 58: 28.77$ & $+29: 47: 53.80$ & $17.4 \pm 1.2$ & $-40.0 \pm 0.6$ & $100.0 \pm 50.0$ & 9.1 & KOIV & $\ldots$ & 8.0 & 0.8 \\
\hline 1RXS J030759.1+302032 & $03: 07: 59.20$ & $+30: 20: 26.05$ & $31.2 \pm 0.6$ & $-66.6 \pm 0.7$ & $75.0 \pm 37.5$ & 7.4 & G5IV & $\ldots$ & 8.3 & 1.1 \\
\hline HD 19668 & $03: 09: 42.28$ & $-09: 34: 46.46$ & $88.0 \pm 1.2$ & $-113.3 \pm 1.1$ & $40.0 \pm 2.0$ & 6.7 & G8/K0V & $\ldots$ & 8.4 & 0.9 \\
\hline 1E $0307.4+1424$ & $03: 10: 12.55$ & $+14: 36: 02.90$ & $-4.0 \pm 1.2$ & $-25.3 \pm 1.2$ & $160.0 \pm 80.0$ & 8.8 & G6V & $\ldots$ & 7.8 & 1.2 \\
\hline V525 Per & $03: 19: 02.76$ & $+48: 10: 59.61$ & $16.4 \pm 4.0$ & $-23.6 \pm 1.4$ & $190.0 \pm 11.0$ & 9.4 & $\mathrm{~K} 2$ & $\alpha \operatorname{Per}$ & 7.9 & 1.0 \\
\hline 1RXS J031907.4+393418 & $03: 19: 07.61$ & $+39: 34: 10.50$ & $27.3 \pm 0.9$ & $-25.3 \pm 1.7$ & $100.0 \pm 50.0$ & 9.5 & K0V & $\ldots$ & 7.8 & 0.8 \\
\hline HE 622 & $03: 24: 49.71$ & $+48: 52: 18.33$ & $22.3 \pm 0.9$ & $-26.3 \pm 0.7$ & $190.0 \pm 11.0$ & 9.6 & G7 & $\alpha \operatorname{Per}$ & 7.9 & 1.1 \\
\hline 1E 0324.1-2012 & $03: 26: 22.05$ & $-20: 01: 48.81$ & $25.0 \pm 1.6$ & $7.4 \pm 1.6$ & $160.0 \pm 80.0$ & 8.9 & $\mathrm{G} 4 \mathrm{~V}$ & $\ldots$ & 7.8 & 1.2 \\
\hline RX J0329.1+0118 & $03: 29: 08.06$ & $+01: 18: 05.66$ & $4.4 \pm 1.3$ & $-4.5 \pm 1.3$ & $100.0 \pm 50.0$ & 9.2 & G0(IV) & $\ldots$ & 7.8 & 0.9 \\
\hline HE 1101 & $03: 35: 08.75$ & $+49: 44: 39.59$ & $20.9 \pm 1.3$ & $-28.5 \pm 0.9$ & $190.0 \pm 11.0$ & 9.3 & G5 & $\alpha$ Per & 7.9 & 1.2 \\
\hline HD 22179 & $03: 35: 29.91$ & $+31: 13: 37.45$ & $42.6 \pm 0.6$ & $-46.0 \pm 0.7$ & $140.0 \pm 70.0$ & 7.4 & G5IV & $\ldots$ & 7.8 & 1.3 \\
\hline HD 23208 & $03: 42: 39.80$ & $-20: 32: 43.80$ & $3.8 \pm 1.5$ & $24.1 \pm 1.0$ & $57.5 \pm 4.7$ & 7.2 & G8V & $\ldots$ & 6.7 & 0.6 \\
\hline Н II 120 & $03: 43: 31.95$ & $+23: 40: 26.61$ & $18.0 \pm 0.7$ & $-46.8 \pm 0.6$ & $133.0 \pm 6.0$ & 9.1 & G5 & Pleiades & 8.1 & 1.1 \\
\hline Н ІІ 2147 & $03: 49: 06.11$ & $+23: 46: 52.49$ & $15.9 \pm 0.9$ & $-43.8 \pm 0.8$ & $133.0 \pm 6.0$ & 8.6 & G7IV & Pleiades & 8.1 & 1.1 \\
\hline 1RXS J035028.0+163121 & $03: 50: 28.40$ & $+16: 31: 15.19$ & $26.2 \pm 1.3$ & $-23.4 \pm 2.1$ & $138.0 \pm 21.0$ & 8.6 & G5IV & $\ldots$ & 7.8 & 1.1 \\
\hline RX J0354.4+0535 & $03: 54: 21.31$ & $+05: 35: 40.77$ & $-1.4 \pm 1.3$ & $-7.6 \pm 1.3$ & $100.0 \pm 50.0$ & 8.7 & G2(V) & $\ldots$ & 8.3 & 1.0 \\
\hline Pels 191 & $03: 54: 25.23$ & $+24: 21: 36.38$ & $17.1 \pm 0.7$ & $-46.8 \pm 0.8$ & $133.0 \pm 6.0$ & 9.1 & G5IV & Pleiades & 8.1 & 1.0 \\
\hline RX J0357.3+1258 & $03: 57: 21.39$ & $+12: 58: 16.83$ & $22.7 \pm 1.8$ & $-21.9 \pm 1.5$ & $149.0 \pm 23.0$ & 9.0 & G0 & $\ldots$ & 7.8 & 1.1 \\
\hline HD 285751 & $04: 23: 41.33$ & $+15: 37: 54.87$ & $8.2 \pm 1.7$ & $-15.8 \pm 1.4$ & $150.0 \pm 75.0$ & 8.8 & $\mathrm{~K} 2(\mathrm{~V})$ & $\ldots$ & 6.8 & 0.9 \\
\hline RX J0442.5+0906 & $04: 42: 32.09$ & $+09: 06: 00.86$ & $28.9 \pm 2.4$ & $-22.3 \pm 2.0$ & $119.0 \pm 21.0$ & 9.1 & G5(V) & $\ldots$ & 7.8 & 1.0 \\
\hline HD 286179 & $04: 57: 00.65$ & $+15: 17: 53.09$ & $-1.8 \pm 1.5$ & $-17.3 \pm 1.4$ & $140.0 \pm 70.0$ & 8.5 & G3(V) & $\ldots$ & 7.3 & 1.2 \\
\hline HD 31950 & $05: 00: 24.31$ & $+15: 05: 25.28$ & $0.3 \pm 1.1$ & $-15.2 \pm 1.1$ & $100.0 \pm 50.0$ & 8.4 & F8 & $\ldots$ & 7.8 & 1.1 \\
\hline HD 35850 & $05: 27: 04.77$ & $-11: 54: 03.38$ & $17.5 \pm 0.7$ & $-49.8 \pm 0.8$ & $27.0 \pm 1.0$ & 4.9 & $\mathrm{~F} 7 / 8 \mathrm{~V}$ & $\ldots$ & 7.5 & 1.2 \\
\hline 1RXS J053650.0+133756 & $05: 36: 50.06$ & $+13: 37: 56.22$ & $4.9 \pm 1.3$ & $-108.8 \pm 1.2$ & $56.0 \pm 28.0$ & 8.1 & K0V & $\ldots$ & 8.3 & 1.1 \\
\hline HD 245567 & $05: 37: 18.44$ & $+13: 34: 52.52$ & $7.5 \pm 0.9$ & $-33.2 \pm 0.9$ & $119.0 \pm 21.0$ & 7.6 & G0V & $\ldots$ & 6.6 & 1.1 \\
\hline SAO 150676 & $05: 40: 20.74$ & $-19: 40: 10.85$ & $19.2 \pm 1.2$ & $-12.9 \pm 1.2$ & $78.0 \pm 30.0$ & 7.5 & $\mathrm{G} 2 \mathrm{~V}$ & $\ldots$ & 7.8 & 1.1 \\
\hline HD 38949 & $05: 48: 20.06$ & $-24: 27: 50.04$ & $-29.8 \pm 1.1$ & $-37.8 \pm 1.2$ & $43.0 \pm 2.0$ & 6.4 & G1V & $\ldots$ & 8.4 & 1.1 \\
\hline HD 43989 & $06: 19: 08.05$ & $-03: 26: 20.39$ & $10.6 \pm 0.9$ & $-43.7 \pm 1.0$ & $50.0 \pm 2.0$ & 6.6 & G0V & $\ldots$ & 7.8 & 1.1 \\
\hline HD 49197 & $06: 49: 21.34$ & $+43: 45: 32.87$ & $-37.6 \pm 0.6$ & $-50.9 \pm 0.6$ & $45.0 \pm 2.0$ & 6.1 & F5 & $\ldots$ & 8.7 & 1.2 \\
\hline RE J0723+20 & $07: 23: 43.58$ & $+20: 24: 58.64$ & $-66.2 \pm 1.8$ & $-230.2 \pm 2.6$ & $24.0 \pm 12.0$ & 6.9 & $\mathrm{~K} 3(\mathrm{~V})$ & $\ldots$ & 8.1 & 0.6 \\
\hline HD 60737 & $07: 38: 16.44$ & $+47: 44: 55.34$ & $-14.2 \pm 1.0$ & $-165.0 \pm 1.0$ & $38.0 \pm 2.0$ & 6.3 & G0 & $\ldots$ & 8.2 & 1.1 \\
\hline HD 70573 & $08: 22: 49.95$ & $+01: 51: 33.58$ & $-49.1 \pm 1.1$ & $-49.7 \pm 1.1$ & $46.0 \pm 23.0$ & 7.2 & $\mathrm{G} 1 / 2 \mathrm{~V}$ & $\ldots$ & 8.0 & 1.0 \\
\hline HD 70516 & $08: 24: 15.66$ & $+44: 56: 58.92$ & $-63.1 \pm 0.9$ & $-178.4 \pm 1.0$ & $37.0 \pm 3.0$ & 6.1 & G0 & $\ldots$ & 7.9 & 1.1 \\
\hline HD 72905 & $08: 39: 11.62$ & $+65: 01: 15.14$ & $-28.9 \pm 1.0$ & $88.5 \pm 1.0$ & $14.0 \pm 0.1$ & 4.2 & G1.5VB & $\ldots$ & 8.3 & 1.0 \\
\hline HD 75393 & $08: 49: 15.35$ & $-15: 33: 53.12$ & $35.8 \pm 1.4$ & $-33.6 \pm 1.2$ & $42.0 \pm 1.0$ & 5.9 & F7V & $\ldots$ & 8.4 & 1.2 \\
\hline HD 82558 & $09: 32: 25.72$ & $-11: 11: 05.00$ & $-248.3 \pm 1.2$ & $35.1 \pm 0.6$ & $18.3 \pm 0.3$ & 5.4 & $\mathrm{~K} 3 \mathrm{~V}$ & $\ldots$ & 8.0 & 0.8 \\
\hline HD 82443 & $09: 32: 43.92$ & $+26: 59: 20.76$ & $-147.5 \pm 0.9$ & $-246.3 \pm 0.5$ & $17.7 \pm 0.3$ & 5.1 & K0V & $\ldots$ & 8.0 & 0.9 \\
\hline SAO 178272 & $09: 59: 08.42$ & $-22: 39: 34.57$ & $-62.8 \pm 1.4$ & $-15.6 \pm 1.7$ & $58.0 \pm 29.0$ & 7.4 & $\mathrm{~K} 2 \mathrm{~V}$ & $\ldots$ & 8.0 & 0.9 \\
\hline HD 90905 & $10: 29: 42.23$ & $+01: 29: 27.82$ & $-150.4 \pm 0.8$ & $-124.1 \pm 0.8$ & $32.0 \pm 1.0$ & 5.5 & G1V & $\ldots$ & 8.3 & 1.2 \\
\hline HD 91782 & $10: 36: 47.84$ & $+47: 43: 12.42$ & $-71.4 \pm 0.6$ & $-81.7 \pm 0.7$ & $56.0 \pm 3.0$ & 6.8 & G0 & $\ldots$ & 8.2 & 1.1 \\
\hline HD 92855 & $10: 44: 00.62$ & $+46: 12: 23.86$ & $-268.8 \pm 1.1$ & $-61.9 \pm 1.2$ & $36.0 \pm 1.0$ & 5.9 & F9V & $\ldots$ & 8.2 & 1.1 \\
\hline HD 93528 & $10: 47: 31.20$ & $-22: 20: 52.80$ & $-122.7 \pm 1.1$ & $-29.4 \pm 0.8$ & $34.9 \pm 1.2$ & 6.5 & KOV & $\ldots$ & 8.0 & 0.9 \\
\hline HD 95188 & $10: 59: 48.28$ & $+25: 17: 23.65$ & $-126.3 \pm 1.4$ & $1.7 \pm 1.3$ & $36.0 \pm 1.0$ & 6.6 & G8V & $\ldots$ & 8.4 & 0.9 \\
\hline HD 101472 & $11: 40: 36.59$ & $-08: 24: 20.32$ & $-20.0 \pm 0.8$ & $-13.8 \pm 0.8$ & $39.0 \pm 2.0$ & 6.1 & F7V & $\ldots$ & 8.4 & 1.1 \\
\hline BPM 87617 & $11: 47: 45.73$ & $+12: 54: 03.31$ & $-71.5 \pm 1.9$ & $-0.4 \pm 1.8$ & $50.0 \pm 25.0$ & 7.8 & K5Ve & $\ldots$ & 8.1 & 0.6 \\
\hline HD 104576 & $12: 02: 39.46$ & $-10: 42: 49.16$ & $32.7 \pm 1.0$ & $-18.4 \pm 0.9$ & $49.0 \pm 3.0$ & 6.7 & G3V & $\ldots$ & 8.2 & 1.0 \\
\hline HD 104860 & $12: 04: 33.71$ & $+66: 20: 11.58$ & $-56.1 \pm 1.4$ & $49.7 \pm 1.4$ & $48.0 \pm 2.0$ & 6.5 & F8 & $\ldots$ & 7.6 & 1.1 \\
\hline HD 107146 & $12: 19: 06.49$ & $+16: 32: 53.91$ & $-175.6 \pm 0.9$ & $-149.5 \pm 1.0$ & $29.0 \pm 1.0$ & 5.5 & $\mathrm{G} 2 \mathrm{~V}$ & $\ldots$ & 8.0 & 1.1 \\
\hline SAO 15880 & $12: 43: 33.36$ & $+60: 00: 53.28$ & $-125.2 \pm 1.4$ & $-66.4 \pm 1.5$ & $60.0 \pm 20.0$ & 7.3 & K0 & $\ldots$ & 8.0 & 1.0 \\
\hline SAO 2085 & $12: 44: 02.88$ & $+85: 26: 56.40$ & $-129.6 \pm 0.8$ & $43.2 \pm 0.9$ & $66.0 \pm 20.0$ & 7.3 & G5 & $\ldots$ & 8.2 & 1.1 \\
\hline HD 111456 & $12: 48: 39.46$ & $+60: 19: 11.40$ & $107.8 \pm 3.1$ & $-30.6 \pm 2.7$ & $24.2 \pm 1.9$ & 4.6 & F5V & $\ldots$ & 8.5 & 1.3 \\
\hline HD 132173 & $14: 58: 30.51$ & $-28: 42: 34.15$ & $-99.9 \pm 1.5$ & $-93.0 \pm 1.7$ & $49.0 \pm 2.0$ & 6.2 & G0V & $\ldots$ & 8.2 & 1.2 \\
\hline HD 139813 & $15: 29: 23.61$ & $+80: 27: 01.08$ & $-218.0 \pm 1.2$ & $105.8 \pm 1.2$ & $22.0 \pm 0.3$ & 5.5 & G5 & $\ldots$ & 8.3 & 0.9 \\
\hline HD 139498 & $15: 39: 24.40$ & $-27: 10: 21.87$ & $-21.8 \pm 1.5$ & $-28.1 \pm 1.5$ & $127.0 \pm 10.0$ & 7.5 & G8(V) & ScoCen & 7.2 & 1.2 \\
\hline HD 142361 & $15: 54: 59.86$ & $-23: 47: 18.26$ & $-29.3 \pm 1.1$ & $-38.8 \pm 1.1$ & $101.0 \pm 14.0$ & 7.0 & G3V & USco & 6.7 & 1.7 \\
\hline HD 143006 & $15: 58: 36.92$ & $-22: 57: 15.35$ & $-10.6 \pm 1.7$ & $-19.5 \pm 1.3$ & $145.0 \pm 40.0$ & 7.1 & G6/8 & USco & 6.7 & 1.8 \\
\hline PZ99 J155847.8-175800 & $15: 58: 47.73$ & $-17: 57: 59.58$ & $-14.8 \pm 3.5$ & $-18.4 \pm 2.8$ & $145.0 \pm 40.0$ & 8.3 & K3 & USco & 6.7 & 1.2 \\
\hline ScoPMS 21 & $16: 01: 25.63$ & $-22: 40: 40.38$ & $-9.4 \pm 2.8$ & $-23.8 \pm 1.7$ & $145.0 \pm 40.0$ & 8.5 & K1IV & USco & 6.7 & 1.0 \\
\hline PZ99 J160158.2-200811 & $16: 01: 58.22$ & $-20: 08: 12.0$ & $-6.8 \pm 2.1$ & $-21.7 \pm 2.3$ & $145.0 \pm 40.0$ & 7.7 & G5 & USco & 6.7 & 1.5 \\
\hline PZ99 J160302.7-180605 & $16: 03: 02.69$ & $-18: 06: 05.06$ & $-11.3 \pm 2.9$ & $-22.7 \pm 1.7$ & $145.0 \pm 40.0$ & 8.7 & K4 & USco & 6.7 & 0.9 \\
\hline
\end{tabular}


Table 1

(Continued)

\begin{tabular}{|c|c|c|c|c|c|c|c|c|c|c|}
\hline Star & $\begin{array}{c}\alpha \\
(\mathrm{J} 2000.0)\end{array}$ & $\begin{array}{c}\delta \\
(\mathrm{J} 2000.0) \\
\end{array}$ & $\begin{array}{c}\mu_{\alpha} \cos \delta \\
\left(\mathrm{mas} \mathrm{yr}^{-1}\right) \\
\end{array}$ & $\begin{array}{c}\mu_{\delta} \\
\left(\text { mas yr }^{-1}\right) \\
\end{array}$ & $\begin{array}{c}d \\
(\mathrm{pc})\end{array}$ & $\begin{array}{c}K_{S} \\
(\mathrm{mag}) \\
\end{array}$ & Sp.T. & Association & $\log$ Age $\mathrm{yr}^{-1}$ & $\begin{array}{l}\text { Mass } \\
\left(M_{\odot}\right) \\
\end{array}$ \\
\hline ScoPMS 27 & $16: 04: 47.76$ & $-19: 30: 23.12$ & $-14.0 \pm 2.3$ & $-20.1 \pm 3.1$ & $145.0 \pm 40.0$ & 8.0 & K2IV & USco & 6.7 & 1.0 \\
\hline ScoPMS 52 & $16: 12: 40.51$ & $-18: 59: 28.31$ & $-8.4 \pm 2.4$ & $-28.5 \pm 4.1$ & $145.0 \pm 40.0$ & 7.5 & K0IV & USco & 6.7 & 1.5 \\
\hline PZ99 J161318.6-221248 & $16: 13: 18.59$ & $-22: 12: 48.96$ & $-9.1 \pm 1.2$ & $-21.0 \pm 1.4$ & $145.0 \pm 40.0$ & 7.4 & G9 & USco & 6.7 & 1.7 \\
\hline PZ99 J161402.1-230101 & $16: 14: 02.12$ & $-23: 01: 02.18$ & $-8.8 \pm 1.7$ & $-22.8 \pm 1.7$ & $145.0 \pm 40.0$ & 8.6 & G4 & USco & 6.7 & 1.0 \\
\hline PZ99 J161411.0-230536 & $16: 14: 11.08$ & $-23: 05: 36.26$ & $-12.1 \pm 1.6$ & $-23.8 \pm 1.9$ & $145.0 \pm 40.0$ & 7.5 & K0 & USco & 6.7 & 1.3 \\
\hline PZ99 J161459.2-275023 & $16: 14: 59.18$ & $-27: 50: 23.06$ & $-12.2 \pm 1.6$ & $-30.5 \pm 5.0$ & $145.0 \pm 40.0$ & 8.7 & G5 & USco & 6.7 & 0.9 \\
\hline PZ99 J161618.0-233947 & $16: 16: 17.95$ & $-23: 39: 47.70$ & $-8.7 \pm 2.0$ & $-26.1 \pm 1.7$ & $145.0 \pm 40.0$ & 8.1 & G7 & USco & 6.7 & 1.3 \\
\hline HD 146516 & $16: 17: 31.39$ & $-23: 03: 36.02$ & $-13.2 \pm 1.2$ & $-17.3 \pm 1.4$ & $145.0 \pm 40.0$ & 8.0 & G0IV & USco & 6.7 & 1.6 \\
\hline ScoPMS 214 & $16: 29: 48.70$ & $-21: 52: 11.91$ & $-5.6 \pm 3.6$ & $-22.1 \pm 1.8$ & $145.0 \pm 40.0$ & 7.8 & K0IV & USco & 6.7 & 1.4 \\
\hline HD 151798 & $16: 50: 05.17$ & $-12: 23: 14.88$ & $-72.8 \pm 1.1$ & $-104.1 \pm 1.3$ & $41.0 \pm 2.0$ & 6.5 & G3V & $\ldots$ & 7.8 & 1.1 \\
\hline HD 165590 & $18: 05: 49.72$ & $+21: 26: 45.60$ & $-21.6 \pm 1.1$ & $-40.5 \pm 0.9$ & $37.7 \pm 1.9$ & 5.4 & G0 & $\ldots$ & 7.5 & 1.1 \\
\hline HD 166181 & $18: 08: 15.67$ & $+29: 41: 28.20$ & $138.1 \pm 1.9$ & $-18.6 \pm 1.7$ & $32.6 \pm 2.2$ & 5.6 & K0 & $\ldots$ & 8.0 & 1.0 \\
\hline HD 170778 & $18: 29: 03.94$ & $+43: 56: 21.54$ & $74.9 \pm 0.9$ & $155.1 \pm 0.9$ & $37.0 \pm 1.0$ & 6.1 & G5 & $\cdots$ & 8.6 & 1.1 \\
\hline HD 171488 & $18: 34: 20.10$ & $+18: 41: 24.20$ & $-20.7 \pm 0.8$ & $-50.9 \pm 0.6$ & $37.2 \pm 1.2$ & 5.8 & G0V & $\ldots$ & 7.5 & 1.1 \\
\hline HD 172649 & $18: 39: 42.11$ & $+37: 59: 35.22$ & $-26.6 \pm 0.6$ & $51.0 \pm 0.7$ & $47.0 \pm 2.0$ & 6.2 & F5 & $\cdots$ & 8.2 & 1.2 \\
\hline HD 187748 & $19: 48: 15.36$ & $+59: 25: 21.36$ & $15.8 \pm 0.6$ & $116.5 \pm 0.5$ & $28.4 \pm 0.4$ & 5.3 & G0 & $\ldots$ & 8.0 & 1.2 \\
\hline HD 191089 & $20: 09: 05.22$ & $-26: 13: 26.63$ & $39.3 \pm 1.1$ & $-68.2 \pm 1.2$ & $54.0 \pm 3.0$ & 6.1 & F5V & $\cdots$ & 8.3 & 1.4 \\
\hline HD 199019 & $20: 49: 29.30$ & $+71: 46: 29.29$ & $139.5 \pm 1.0$ & $100.3 \pm 1.1$ & $35.0 \pm 1.0$ & 6.5 & G5 & $\ldots$ & 8.4 & 0.9 \\
\hline HD 200746 & $21: 05: 07.95$ & $+07: 56: 43.59$ & $3.6 \pm 1.1$ & $-94.7 \pm 1.7$ & $44.0 \pm 6.0$ & 6.4 & G5 & $\ldots$ & 8.6 & 1.0 \\
\hline HD 203030 & $21: 18: 58.22$ & $+26: 13: 50.05$ & $131.3 \pm 1.6$ & $8.6 \pm 0.9$ & $41.0 \pm 2.0$ & 6.7 & G8V & $\ldots$ & 8.6 & 1.0 \\
\hline HD 209393 & $22: 02: 05.38$ & $+44: 20: 35.47$ & $38.7 \pm 1.2$ & $30.9 \pm 1.2$ & $34.0 \pm 1.0$ & 6.3 & G5 & $\ldots$ & 8.6 & 1.0 \\
\hline HD 209779 & $22: 06: 05.32$ & $-05: 21: 29.15$ & $160.4 \pm 0.9$ & $-59.3 \pm 0.9$ & $36.0 \pm 1.0$ & 5.9 & $\mathrm{G} 2 \mathrm{~V}$ & $\ldots$ & 8.6 & 1.1 \\
\hline V383 Lac & $22: 20: 07.03$ & $+49: 30: 11.67$ & $93.4 \pm 1.2$ & $5.0 \pm 1.2$ & $50.0 \pm 25.0$ & 6.5 & K0VIV & $\ldots$ & 7.8 & 1.0 \\
\hline HD 217343 & $23: 00: 19.29$ & $-26: 09: 13.48$ & $108.5 \pm 1.3$ & $-162.1 \pm 1.4$ & $32.0 \pm 1.0$ & 5.9 & G3V & $\ldots$ & 7.6 & 1.0 \\
\hline HD 218738 & $23: 09: 57.23$ & $+47: 57: 30.00$ & $147.1 \pm 6.8$ & $12.4 \pm 5.6$ & $25.3 \pm 4.9$ & 5.7 & $\mathrm{dK} 2+\mathrm{dK} 2$ & $\cdots$ & 8.5 & 0.9 \\
\hline HD 218739 & $23: 09: 58.87$ & $+47: 57: 33.90$ & $154.2 \pm 2.0$ & $-1.1 \pm 1.7$ & $29.4 \pm 2.0$ & 5.7 & G1V & $\cdots$ & 8.5 & 1.1 \\
\hline HD 219498 & $23: 16: 05.02$ & $+22: 10: 34.98$ & $82.0 \pm 0.9$ & $-30.5 \pm 1.0$ & $60.0 \pm 30.0$ & 7.4 & G5 & $\ldots$ & 8.4 & 1.5 \\
\hline
\end{tabular}

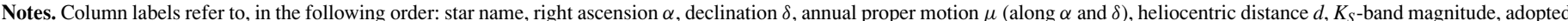

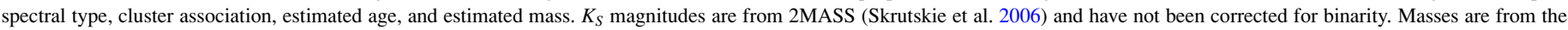

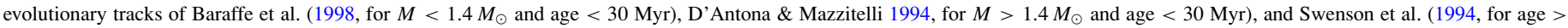
$30 \mathrm{Myr}$ ). The mass estimates have been corrected for binarity.

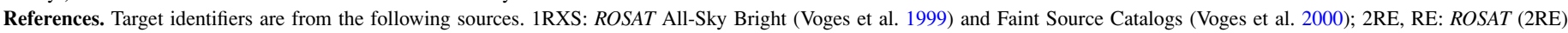

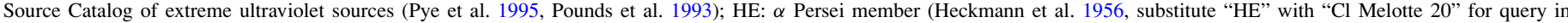

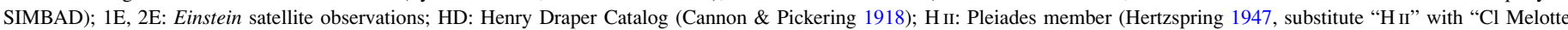

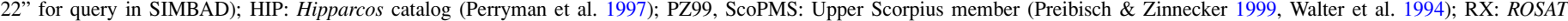

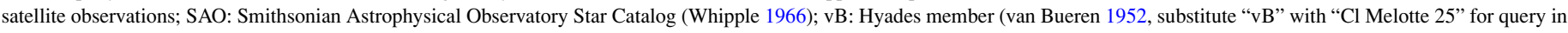
SIMBAD).

(This table is also available in a machine-readable form in the online journal.)
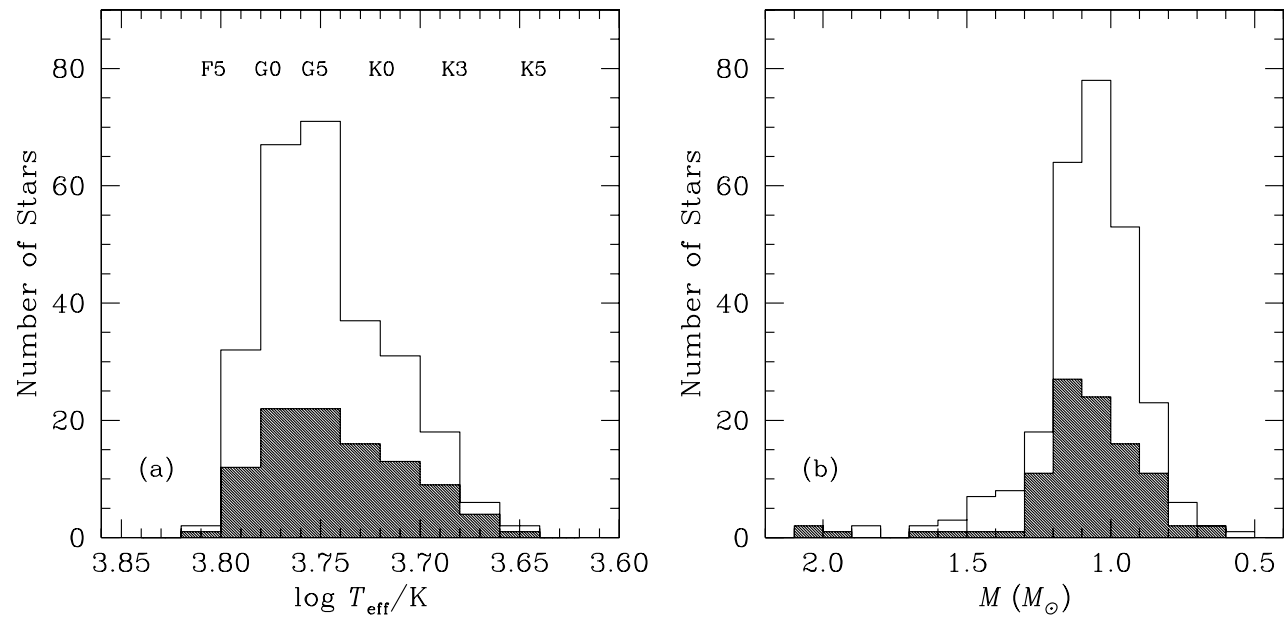

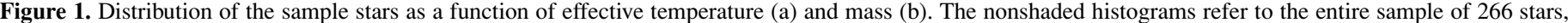

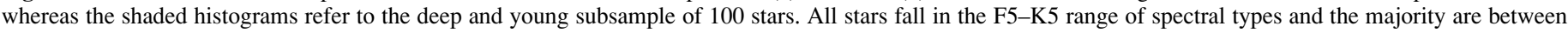
$0.7 M_{\odot}$ and $1.3 M_{\odot}$.

\section{OBSERVATIONS, DATA REDUCTION, AND CALIBRATION}

\subsection{Observing Strategy}

A complete description of the observing strategy of our AO survey was given in Metchev \& Hillenbrand (2004) and in Metchev (2006). Here, we briefly review the approach and summarize the survey observations.

The full sample of 266 stars was observed in the near-IR (NIR) with $\mathrm{AO}$ at the Palomar and Keck II telescopes on 47 clear nights over the course of three years: between 2002 January 31 and 2005 January 24. Additional astrometric follow- 
Table 2

Shallow Sample

\begin{tabular}{|c|c|c|c|c|c|c|c|c|c|c|}
\hline Star & $\begin{array}{c}\alpha \\
(\mathrm{J} 2000.0)\end{array}$ & $\begin{array}{c}\delta \\
(\mathrm{J} 2000.0) \\
\end{array}$ & $\begin{array}{c}\mu_{\alpha} \cos \delta \\
\left(\operatorname{mas~yr}^{-1}\right)\end{array}$ & $\begin{array}{c}\mu_{\delta} \\
\left(\operatorname{mas~yr}^{-1}\right)\end{array}$ & $\begin{array}{c}d \\
(\mathrm{pc})\end{array}$ & $\begin{array}{c}K_{S} \\
(\mathrm{mag})\end{array}$ & Sp.T. & Association & $\log$ Age $\mathrm{yr}^{-1}$ & $\begin{array}{l}\text { Mass } \\
\left(M_{\odot}\right) \\
\end{array}$ \\
\hline HD 224873 & 00:01:23.66 & $+39: 36: 38.12$ & $-28.7 \pm 0.6$ & $-43.3 \pm 0.7$ & $49.0 \pm 5.0$ & 6.7 & K0 & & 8.5 & 0.9 \\
\hline HD 6963 & 01:10:41.91 & $+42: 55: 54.50$ & $-154.6 \pm 0.9$ & $-198.5 \pm 0.9$ & $27.0 \pm 1.0$ & 5.9 & G7V & $\ldots$ & 9.0 & 0.9 \\
\hline HD 8467 & $01: 24: 28.00$ & $+39: 03: 43.55$ & $210.6 \pm 1.8$ & $-26.6 \pm 1.0$ & $31.0 \pm 1.0$ & 6.6 & G5 & $\ldots$ & 9.3 & 0.8 \\
\hline HD 8941 & $01: 28: 24.36$ & $+17: 04: 45.20$ & $118.3 \pm 0.7$ & $-34.8 \pm 0.7$ & $50.0 \pm 2.0$ & 5.4 & F8IV-V & $\ldots$ & 9.2 & 1.5 \\
\hline HD 9472 & 01:33:19.03 & $+23: 58: 32.19$ & $0.0 \pm 1.0$ & $28.4 \pm 0.9$ & $33.0 \pm 1.0$ & 6.0 & G0 & $\ldots$ & 8.9 & 1.0 \\
\hline RE J0137+18A & 01:37:39.41 & $+18: 35: 33.16$ & $65.8 \pm 1.9$ & $-46.0 \pm 2.5$ & $64.0 \pm 8.0$ & 6.7 & $\mathrm{~K} 3 \mathrm{Ve}$ & $\ldots$ & 6.8 & 0.6 \\
\hline HD 11850 & $01: 56: 47.27$ & $+23: 03: 04.09$ & $-83.8 \pm 1.0$ & $-18.1 \pm 1.0$ & $33.0 \pm 1.0$ & 6.2 & G5 & $\ldots$ & 8.8 & 1.0 \\
\hline HD 13382 & $02: 11: 23.15$ & $+21: 22: 38.39$ & $273.1 \pm 0.8$ & $-12.6 \pm 0.7$ & $33.0 \pm 1.0$ & 5.8 & G5V & $\ldots$ & 8.7 & 1.1 \\
\hline HD 13507 & $02: 12: 55.00$ & $+40: 40: 06.00$ & $56.9 \pm 1.3$ & $-99.2 \pm 1.3$ & $26.0 \pm 1.0$ & 5.6 & G5V & $\ldots$ & 8.9 & 1.0 \\
\hline HD 13531 & $02: 13: 13.35$ & $+40: 30: 27.34$ & $57.6 \pm 1.0$ & $-96.4 \pm 1.0$ & $26.0 \pm 1.0$ & 5.7 & G7V & $\ldots$ & 8.7 & 1.0 \\
\hline HD 13974 & $02: 17: 03.23$ & $+34: 13: 27.32$ & $1153.8 \pm 0.8$ & $-245.1 \pm 0.8$ & $11.0 \pm 0.1$ & 3.2 & G0V & $\ldots$ & 9.2 & 1.1 \\
\hline 1RXS J025223.5+372914 & $02: 52: 24.73$ & $+37: 28: 51.83$ & $22.5 \pm 0.7$ & $-24.5 \pm 1.0$ & $170.0 \pm 85.0$ & 9.1 & G5IV & $\ldots$ & 8.3 & 1.1 \\
\hline 2RE J0255+474 & $02: 55: 43.60$ & $+47: 46: 47.58$ & $79.8 \pm 0.6$ & $-76.1 \pm 0.7$ & $50.0 \pm 25.0$ & 7.2 & $\mathrm{~K} 5 \mathrm{Ve}$ & $\ldots$ & 7.9 & 0.8 \\
\hline HD 18940 & $03: 03: 28.65$ & $+23: 03: 41.19$ & $111.4 \pm 0.8$ & $-0.7 \pm 0.7$ & $34.0 \pm 1.0$ & 5.5 & G0 & $\ldots$ & 8.9 & 1.0 \\
\hline HD 19019 & 03:03:50.82 & $+06: 07: 59.82$ & $231.8 \pm 1.8$ & $50.7 \pm 1.7$ & $31.0 \pm 1.0$ & 5.6 & F8 & $\ldots$ & 9.2 & 1.1 \\
\hline HD 19632 & 03:08:52.45 & $-24: 53: 15.55$ & $226.7 \pm 1.3$ & $136.3 \pm 1.3$ & $30.0 \pm 1.0$ & 5.7 & $\mathrm{G} 3 / 5 \mathrm{~V}$ & $\ldots$ & 8.6 & 1.1 \\
\hline vB 1 & $03: 17: 26.39$ & $+07: 39: 20.90$ & $167.2 \pm 1.3$ & $-6.4 \pm 1.4$ & $43.1 \pm 0.6$ & 6.0 & F8 & Hyades & 8.8 & 1.2 \\
\hline HE 350 & $03: 17: 36.93$ & $+48: 50: 08.50$ & $23.2 \pm 0.8$ & $-23.0 \pm 0.9$ & $190.0 \pm 11.0$ & 9.3 & G2 & $\alpha$ Per & 7.9 & 1.2 \\
\hline HE 373 & $03: 18: 27.39$ & $+47: 21: 15.42$ & $29.0 \pm 0.7$ & $-26.8 \pm 2.0$ & $190.0 \pm 11.0$ & 9.4 & G8 & $\alpha$ Per & 7.9 & 1.2 \\
\hline HE 389 & $03: 18: 50.31$ & $+49: 43: 52.19$ & $22.5 \pm 0.9$ & $-23.9 \pm 0.7$ & $190.0 \pm 11.0$ & 9.5 & G0 & $\alpha$ Per & 7.9 & 1.1 \\
\hline HE 696 & $03: 26: 19.36$ & $+49: 13: 32.54$ & $19.8 \pm 0.7$ & $-25.0 \pm 0.7$ & $190.0 \pm 11.0$ & 9.7 & G3 & $\alpha$ Per & 7.9 & 1.0 \\
\hline HE 699 & $03: 26: 22.22$ & $+49: 25: 37.52$ & $22.4 \pm 0.8$ & $-24.5 \pm 0.7$ & $190.0 \pm 11.0$ & 9.4 & G3 & $\alpha$ Per & 7.9 & 1.1 \\
\hline HE 750 & $03: 27: 37.79$ & $+48: 59: 28.78$ & $22.0 \pm 0.7$ & $-25.6 \pm 0.7$ & $190.0 \pm 11.0$ & 9.1 & F5 & $\alpha$ Per & 7.9 & 1.2 \\
\hline HE 767 & $03: 27: 55.02$ & $+49: 45: 37.16$ & $21.1 \pm 0.6$ & $-26.0 \pm 0.6$ & $190.0 \pm 11.0$ & 9.2 & F6 & $\alpha$ Per & 7.9 & 1.2 \\
\hline HE 848 & $03: 29: 26.24$ & $+48: 12: 11.74$ & $22.2 \pm 0.6$ & $-26.4 \pm 0.6$ & $190.0 \pm 11.0$ & 8.5 & F9V & $\alpha$ Per & 7.9 & 1.3 \\
\hline HE 935 & 03:31:28.99 & $+48: 59: 28.37$ & $21.3 \pm 0.9$ & $-26.6 \pm 0.6$ & $190.0 \pm 11.0$ & 8.5 & $\mathrm{~F} 9.5 \mathrm{~V}$ & $\alpha$ Per & 7.9 & 1.2 \\
\hline HE 1234 & 03:39:02.91 & $+51: 36: 37.11$ & $21.4 \pm 0.8$ & $-33.7 \pm 0.7$ & $190.0 \pm 11.0$ & 8.9 & G4 & $\alpha$ Per & 7.9 & 1.3 \\
\hline HD 22879 & $03: 40: 22.08$ & $-03: 13: 00.86$ & $691.6 \pm 1.1$ & $-212.8 \pm 1.1$ & $24.0 \pm 1.0$ & 5.2 & $\mathrm{~F} 7 / 8 \mathrm{~V}$ & $\ldots$ & 9.3 & 0.8 \\
\hline H II 102 & $03: 43: 24.54$ & $+23: 13: 33.30$ & $17.1 \pm 0.6$ & $-43.7 \pm 0.6$ & $133.0 \pm 6.0$ & 8.7 & G6 & Pleiades & 8.1 & 1.1 \\
\hline Н II 152 & $03: 43: 37.73$ & $+23: 32: 09.59$ & $19.5 \pm 0.7$ & $-46.9 \pm 1.0$ & $133.0 \pm 6.0$ & 9.1 & G4 & Pleiades & 8.1 & 1.0 \\
\hline Н II 174 & $03: 43: 48.33$ & $+25: 00: 15.83$ & $18.8 \pm 1.1$ & $-47.0 \pm 0.9$ & $133.0 \pm 6.0$ & 9.4 & $\mathrm{~K} 1$ & Pleiades & 8.1 & 0.9 \\
\hline H II 173 & $03: 43: 48.41$ & $+25: 11: 24.19$ & $20.4 \pm 0.8$ & $-48.4 \pm 0.7$ & $133.0 \pm 6.0$ & 8.8 & K0 & Pleiades & 8.1 & 1.1 \\
\hline Н II 250 & 03:44:04.24 & $+24: 59: 23.40$ & $20.1 \pm 1.0$ & $-49.4 \pm 0.7$ & $133.0 \pm 6.0$ & 9.1 & G3 & Pleiades & 8.1 & 1.0 \\
\hline Н II 314 & 03:44:20.09 & $+24: 47: 46.16$ & $18.2 \pm 0.7$ & $-49.8 \pm 0.8$ & $133.0 \pm 6.0$ & 8.9 & G3 & Pleiades & 8.1 & 1.1 \\
\hline 1RXS J034423.3+281224 & $03: 44: 24.25$ & $+28: 12: 23.07$ & $46.4 \pm 0.7$ & $-50.6 \pm 0.6$ & $49.0 \pm 10.0$ & 7.2 & G7V & $\ldots$ & 7.8 & 0.8 \\
\hline Н II 514 & 03:45:04.01 & $+25: 15: 28.23$ & $17.3 \pm 0.7$ & $-46.3 \pm 0.6$ & $133.0 \pm 6.0$ & 9.0 & G4 & Pleiades & 8.1 & 1.1 \\
\hline H II 571 & $03: 45: 15.35$ & $+25: 17: 22.11$ & $15.1 \pm 0.9$ & $-48.5 \pm 0.9$ & $133.0 \pm 6.0$ & 9.2 & G9 & Pleiades & 8.1 & 1.0 \\
\hline H II 1015 & $03: 46: 27.35$ & $+25: 08: 07.97$ & $18.6 \pm 0.7$ & $-48.5 \pm 0.9$ & $133.0 \pm 6.0$ & 9.0 & G1 & Pleiades & 8.1 & 1.1 \\
\hline H II 1101 & $03: 46: 38.78$ & $+24: 57: 34.61$ & $18.4 \pm 0.8$ & $-48.1 \pm 0.7$ & $133.0 \pm 6.0$ & 8.8 & G4 & Pleiades & 8.1 & 1.1 \\
\hline H II 1182 & $03: 46: 47.06$ & $+22: 54: 52.48$ & $18.4 \pm 0.6$ & $-45.6 \pm 0.7$ & $133.0 \pm 6.0$ & 8.9 & G1 & Pleiades & 8.1 & 1.1 \\
\hline H II 1200 & $03: 46: 50.54$ & $+23: 14: 21.06$ & $17.3 \pm 0.6$ & $-40.2 \pm 0.7$ & $133.0 \pm 6.0$ & 8.5 & F6 & Pleiades & 8.1 & 1.3 \\
\hline Н II 1776 & $03: 48: 17.70$ & $+25: 02: 52.29$ & $19.0 \pm 1.0$ & $-47.1 \pm 1.0$ & $133.0 \pm 6.0$ & 9.2 & G5 & Pleiades & 8.1 & 1.0 \\
\hline H II 2106 & $03: 48: 58.49$ & $+23: 12: 04.33$ & $16.5 \pm 1.3$ & $-44.9 \pm 1.1$ & $133.0 \pm 6.0$ & 9.4 & K1 & Pleiades & 8.1 & 0.9 \\
\hline RX J0348.9+0110 & $03: 48: 58.76$ & $+01: 10: 53.99$ & $35.1 \pm 1.6$ & $-22.1 \pm 1.2$ & $100.0 \pm 50.0$ & 8.3 & $\mathrm{~K} 3(\mathrm{~V}) / \mathrm{E}$ & $\ldots$ & 8.2 & 0.9 \\
\hline Н II 2278 & $03: 49: 25.70$ & $+24: 56: 15.43$ & $18.4 \pm 0.9$ & $-47.0 \pm 0.8$ & $133.0 \pm 6.0$ & 8.8 & K0 & Pleiades & 8.1 & 0.9 \\
\hline Н II 2506 & 03:49:56.49 & $+23: 13: 07.01$ & $17.6 \pm 0.7$ & $-43.9 \pm 0.6$ & $133.0 \pm 6.0$ & 8.8 & F9 & Pleiades & 8.1 & 1.2 \\
\hline H II 2644 & 03:50:20.90 & $+24: 28: 00.22$ & $19.8 \pm 0.8$ & $-46.8 \pm 0.9$ & $133.0 \pm 6.0$ & 9.3 & G5 & Pleiades & 8.1 & 1.0 \\
\hline Н II 2786 & 03:50:40.08 & $+23: 55: 58.94$ & $17.6 \pm 0.7$ & $-45.2 \pm 1.0$ & $133.0 \pm 6.0$ & 8.9 & F9 & Pleiades & 8.1 & 1.1 \\
\hline H II 2881 & 03:50:54.32 & $+23: 50: 05.52$ & $17.7 \pm 0.7$ & $-46.9 \pm 1.1$ & $133.0 \pm 6.0$ & 9.1 & $\mathrm{~K} 2$ & Pleiades & 8.1 & 0.8 \\
\hline Н II 3097 & 03:51:40.44 & $+24: 58: 59.41$ & $17.5 \pm 0.7$ & $-46.1 \pm 1.0$ & $133.0 \pm 6.0$ & 9.1 & G6 & Pleiades & 8.1 & 1.1 \\
\hline Н II 3179 & $03: 51: 56.86$ & $+23: 54: 06.98$ & $19.2 \pm 0.6$ & $-46.5 \pm 0.8$ & $133.0 \pm 6.0$ & 8.6 & F8 & Pleiades & 8.1 & 1.2 \\
\hline HD 285281 & 04:00:31.07 & $+19: 35: 20.70$ & $2.7 \pm 1.1$ & $-12.9 \pm 1.2$ & $49.0 \pm 11.0$ & 7.6 & K1 & $\ldots$ & 7.0 & 0.4 \\
\hline HD 284135 & $04: 05: 40.58$ & $+22: 48: 12.14$ & $6.0 \pm 0.6$ & $-14.9 \pm 0.6$ & $140.0 \pm 70.0$ & 7.8 & $\mathrm{G} 3(\mathrm{~V})$ & $\ldots$ & 6.8 & 1.1 \\
\hline HD 281691 & 04:09:09.74 & $+29: 01: 30.55$ & $19.9 \pm 0.7$ & $-36.3 \pm 1.0$ & $140.0 \pm 70.0$ & 8.4 & $\mathrm{~K} 1(\mathrm{~V})$ & $\ldots$ & 7.8 & 1.1 \\
\hline HD 26182 & 04:10:04.69 & $+36: 39: 12.14$ & $23.8 \pm 0.7$ & $-36.7 \pm 0.7$ & $100.0 \pm 50.0$ & 7.8 & G0V & $\ldots$ & 7.8 & 1.1 \\
\hline HD 284266 & $04: 15: 22.92$ & $+20: 44: 16.93$ & $1.8 \pm 1.0$ & $-13.6 \pm 0.7$ & $140.0 \pm 70.0$ & 8.6 & $\mathrm{~K} 0(\mathrm{~V})$ & $\ldots$ & 7.3 & 1.1 \\
\hline HD 26990 & $04: 16: 16.50$ & $+07: 09: 34.15$ & $-85.6 \pm 1.5$ & $-52.1 \pm 1.5$ & $35.0 \pm 2.0$ & 5.9 & $\mathrm{G} 0(\mathrm{~V})$ & $\ldots$ & 8.9 & 0.9 \\
\hline HD 27466 & 04:19:57.08 & $-04: 26: 19.60$ & $-58.6 \pm 1.2$ & $-37.0 \pm 1.2$ & $36.0 \pm 1.0$ & 6.3 & G5V & $\ldots$ & 9.2 & 1.0 \\
\hline vB 39 & $04: 22: 44.74$ & $+16: 47: 27.56$ & $173.3 \pm 11.5$ & $4.7 \pm 10.2$ & $39.3 \pm 3.5$ & 6.2 & G4V & Hyades & 8.8 & 1.1 \\
\hline vB 49 & $04: 24: 12.78$ & $+16: 22: 44.22$ & $87.6 \pm 1.3$ & $-21.9 \pm 1.2$ & $57.5 \pm 1.0$ & 6.8 & G0V & Hyades & 8.8 & 1.1 \\
\hline vB 52 & $04: 24: 28.33$ & $+16: 53: 10.32$ & $113.1 \pm 1.4$ & $-23.3 \pm 1.2$ & $44.8 \pm 0.8$ & 6.3 & $\mathrm{G} 2 \mathrm{~V}$ & Hyades & 8.8 & 1.1 \\
\hline vB 176 & $04: 25: 47.56$ & $+18: 01: 02.20$ & $102.6 \pm 2.2$ & $-29.9 \pm 3.2$ & $48.0 \pm 1.0$ & 6.8 & $\mathrm{~K} 2 \mathrm{~V}$ & Hyades & 8.8 & 0.8 \\
\hline vB 63 & $04: 26: 24.61$ & $+16: 51: 11.84$ & $106.7 \pm 1.3$ & $-24.5 \pm 1.2$ & $46.9 \pm 1.0$ & 6.4 & G1V & Hyades & 8.8 & 1.1 \\
\hline vB 64 & $04: 26: 40.11$ & $+16: 44: 48.78$ & $107.0 \pm 1.1$ & $-26.8 \pm 1.1$ & $46.4 \pm 0.9$ & 6.5 & $\mathrm{G} 2+$ & Hyades & 8.8 & 1.1 \\
\hline vB 66 & $04: 27: 46.07$ & $+11: 44: 11.07$ & $110.1 \pm 1.3$ & $-13.2 \pm 1.2$ & $44.6 \pm 0.9$ & 6.2 & F8 & Hyades & 8.8 & 1.2 \\
\hline
\end{tabular}


Table 2

(Continued)

\begin{tabular}{|c|c|c|c|c|c|c|c|c|c|c|}
\hline Star & $\begin{array}{c}\alpha \\
(\mathrm{J} 2000.0)\end{array}$ & $\begin{array}{c}\delta \\
(\mathrm{J} 2000.0)\end{array}$ & 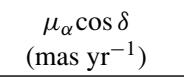 & $\begin{array}{c}\mu_{\delta} \\
\left(\operatorname{mas~yr}^{-1}\right)\end{array}$ & $\begin{array}{c}d \\
(\mathrm{pc})\end{array}$ & $\begin{array}{c}K_{S} \\
\text { (mag) }\end{array}$ & Sp.T. & Association & $\log$ Age $\mathrm{yr}^{-1}$ & $\begin{array}{l}\text { Mass } \\
\left(M_{\odot}\right) \\
\end{array}$ \\
\hline vB 73 & $04: 28: 48.29$ & $+17: 17: 07.84$ & $110.1 \pm 1.1$ & $-28.9 \pm 1.0$ & $44.5 \pm 0.8$ & 6.4 & G2V & Hyades & 8.8 & 1.1 \\
\hline vB 79 & $04: 29: 31.61$ & $+17: 53: 35.46$ & $106.7 \pm 1.1$ & $-31.4 \pm 1.1$ & $45.6 \pm 0.8$ & 7.1 & K0V & Hyades & 8.8 & 0.9 \\
\hline vB 180 & $04: 29: 57.73$ & $+16: 40: 22.23$ & $106.2 \pm 1.1$ & $-27.1 \pm 1.1$ & $46.0 \pm 0.8$ & 7.1 & K1V & Hyades & 8.8 & 0.9 \\
\hline vB 88 & $04: 31: 29.35$ & $+13: 54: 12.55$ & $90.0 \pm 1.2$ & $-16.0 \pm 1.2$ & $53.1 \pm 1.3$ & 6.5 & F9V & Hyades & 8.8 & 1.2 \\
\hline 1RXS J043243.2-152003 & $04: 32: 43.51$ & $-15: 20: 11.39$ & $2.3 \pm 1.1$ & $14.2 \pm 1.1$ & $140.0 \pm 70.0$ & 8.6 & G4V & & 6.6 & 0.8 \\
\hline vB 91 & $04: 32: 50.12$ & $+16: 00: 20.96$ & $103.2 \pm 1.0$ & $-25.9 \pm 1.0$ & $45.9 \pm 0.6$ & 6.8 & G7 & Hyades & 8.8 & 0.8 \\
\hline vB 92 & $04: 32: 59.45$ & $+15: 49: 08.37$ & $99.1 \pm 1.2$ & $-24.1 \pm 1.2$ & $47.8 \pm 0.8$ & 6.9 & G7 & Hyades & 8.8 & 1.0 \\
\hline vB 93 & $04: 33: 37.97$ & $+16: 45: 44.96$ & $99.0 \pm 1.1$ & $-22.9 \pm 1.2$ & $48.3 \pm 0.7$ & 7.4 & G7 & Hyades & 8.8 & 0.9 \\
\hline vB 96 & $04: 33: 58.54$ & $+15: 09: 49.04$ & $101.9 \pm 1.3$ & $-29.4 \pm 1.3$ & $45.4 \pm 0.8$ & 6.5 & G5 & Hyades & 8.8 & 0.9 \\
\hline RX J0434.3+0226 & $04: 34: 19.54$ & $+02: 26: 26.10$ & $18.0 \pm 2.0$ & $-16.4 \pm 1.9$ & $161.0 \pm 24.0$ & 9.5 & $\mathrm{~K} 4 \mathrm{e}$ & $\ldots$ & 7.8 & 1.0 \\
\hline vB 183 & $04: 34: 32.18$ & $+15: 49: 39.23$ & $91.0 \pm 1.0$ & $-20.0 \pm 1.0$ & $51.7 \pm 0.8$ & 7.6 & G7 & Hyades & 8.8 & 0.9 \\
\hline vB 97 & $04: 34: 35.31$ & $+15: 30: 16.56$ & $98.1 \pm 1.0$ & $-26.7 \pm 1.1$ & $47.2 \pm 0.9$ & 6.4 & F8:V: & Hyades & 8.8 & 1.1 \\
\hline vB 99 & $04: 36: 05.27$ & $+15: 41: 02.60$ & $95.0 \pm 1.0$ & $-23.1 \pm 1.2$ & $48.7 \pm 0.7$ & 7.4 & G7 & Hyades & 8.8 & 0.9 \\
\hline vB 106 & 04:38:57.31 & $+14: 06: 20.16$ & $99.5 \pm 0.9$ & $-24.4 \pm 1.1$ & $44.6 \pm 0.9$ & 6.4 & G5 & Hyades & 8.8 & 1.1 \\
\hline HD 282346 & 04:39:31.00 & $+34: 07: 44.43$ & $31.3 \pm 0.7$ & $-53.8 \pm 0.9$ & $71.0 \pm 14.0$ & 7.4 & G8V & $\ldots$ & 8.0 & 1.0 \\
\hline vB 142 & $04: 46: 30.38$ & $+15: 28: 19.38$ & $87.8 \pm 1.1$ & $-23.9 \pm 1.1$ & $48.2 \pm 1.1$ & 6.7 & G5 & Hyades & 8.8 & 1.1 \\
\hline vB 143 & $04: 51: 23.22$ & $+15: 26: 00.45$ & $66.7 \pm 1.2$ & $-17.2 \pm 1.2$ & $61.1 \pm 1.9$ & 6.7 & F8 & Hyades & 8.8 & 1.2 \\
\hline HD 286264 & 05:00:49.28 & $+15: 27: 00.68$ & $20.0 \pm 1.4$ & $-59.0 \pm 1.4$ & $71.0 \pm 11.0$ & 7.6 & $\mathrm{~K} 2 \mathrm{IV}$ & $\ldots$ & 7.3 & 1.0 \\
\hline HD 32850 & $05: 06: 42.21$ & $+14: 26: 46.42$ & $282.8 \pm 1.1$ & $-239.9 \pm 1.1$ & $24.0 \pm 1.0$ & 5.7 & G9V & $\ldots$ & 9.1 & 0.9 \\
\hline 1RXS J051111.1+281353 & $05: 11: 10.53$ & $+28: 13: 50.38$ & $6.0 \pm 0.8$ & $-24.0 \pm 0.7$ & $139.0 \pm 10.0$ & 7.8 & K0V & $\ldots$ & 6.7 & 1.1 \\
\hline HD 36869 & $05: 34: 09.16$ & $-15: 17: 03.20$ & $23.9 \pm 3.4$ & $-21.8 \pm 2.9$ & $72.0 \pm 21.0$ & 6.9 & G2V & $\cdots$ & 7.5 & 1.2 \\
\hline HD 37216 & $05: 39: 52.33$ & $+52: 53: 50.83$ & $-10.0 \pm 1.3$ & $-141.4 \pm 1.4$ & $28.0 \pm 1.0$ & 6.0 & G5 & $\ldots$ & 8.8 & 0.9 \\
\hline HD 37006 & $05: 46: 11.89$ & $+78: 15: 22.61$ & $-45.9 \pm 1.4$ & $70.7 \pm 1.4$ & $35.0 \pm 1.0$ & 6.5 & G0 & $\ldots$ & 8.9 & 0.9 \\
\hline HD 38529 & $05: 46: 34.92$ & $+01: 10: 05.31$ & $-79.3 \pm 0.9$ & $-140.6 \pm 1.0$ & $42.0 \pm 2.0$ & 4.2 & G8III/IV & $\ldots$ & 9.7 & 1.6 \\
\hline HD 61994 & $07: 47: 30.61$ & $+70: 12: 23.97$ & $-88.0 \pm 1.0$ & $-148.7 \pm 1.1$ & $28.0 \pm 2.0$ & 5.3 & G6V & $\ldots$ & 9.0 & 1.1 \\
\hline HD 64324 & $07: 54: 48.47$ & $+34: 37: 11.42$ & $-120.5 \pm 1.0$ & $-173.4 \pm 1.1$ & $35.0 \pm 1.0$ & 6.2 & G0 & $\ldots$ & 9.1 & 1.0 \\
\hline HD 66751 & 08:10:20.51 & $+69: 43: 30.21$ & $165.9 \pm 1.0$ & $116.1 \pm 1.1$ & $29.0 \pm 1.0$ & 5.1 & F8V & $\ldots$ & 9.2 & 1.2 \\
\hline HD 69076 & $08: 15: 07.73$ & $-06: 55: 08.23$ & $-11.6 \pm 0.9$ & $-159.3 \pm 0.9$ & $34.0 \pm 1.0$ & 6.4 & $\mathrm{~K} 0 \mathrm{~V}$ & $\ldots$ & 9.3 & 0.9 \\
\hline HD 71974 & $08: 31: 35.05$ & $+34: 57: 58.44$ & $-5.9 \pm 1.4$ & $16.9 \pm 1.5$ & $29.0 \pm 1.0$ & 5.5 & G5 & $\ldots$ & 8.9 & 0.9 \\
\hline HD 72687 & $08: 33: 15.39$ & $-29: 57: 23.66$ & $-40.5 \pm 1.3$ & $19.8 \pm 1.0$ & $46.0 \pm 2.0$ & 6.7 & G5V & $\cdots$ & 8.6 & 1.0 \\
\hline HD 72760 & $08: 34: 31.65$ & $-00: 43: 33.80$ & $-194.3 \pm 1.1$ & $23.4 \pm 0.8$ & $21.8 \pm 0.5$ & 5.4 & G5 & $\ldots$ & 8.8 & 0.9 \\
\hline HD 73668 & 08:39:43.81 & $+05: 45: 51.59$ & $177.6 \pm 1.5$ & $-298.4 \pm 1.6$ & $36.0 \pm 2.0$ & 5.8 & G1V & $\ldots$ & 9.4 & 1.1 \\
\hline HIP 42491 & 08:39:44.69 & $+05: 46: 14.00$ & $173.9 \pm 3.1$ & $-297.2 \pm 3.0$ & $37.0 \pm 8.0$ & 6.5 & G5 & $\ldots$ & 9.2 & 0.9 \\
\hline HD 75302 & $08: 49: 12.53$ & $+03: 29: 05.25$ & $-147.8 \pm 1.1$ & $60.2 \pm 1.1$ & $30.0 \pm 1.0$ & 5.8 & G5V & $\ldots$ & 9.1 & 1.0 \\
\hline HD 76218 & $08: 55: 55.68$ & $+36: 11: 46.40$ & $-25.4 \pm 0.6$ & $-12.4 \pm 0.7$ & $26.0 \pm 1.0$ & 5.8 & G9-V & $\ldots$ & 8.7 & 0.9 \\
\hline HD 77407 & 09:03:27.08 & $+37: 50: 27.72$ & $-80.2 \pm 1.2$ & $-168.0 \pm 1.3$ & $30.0 \pm 1.0$ & 5.4 & G0(V) & $\ldots$ & 7.5 & 1.0 \\
\hline HD 78899 & $09: 12: 28.27$ & $+49: 12: 24.90$ & $-49.7 \pm 1.2$ & $-176.5 \pm 0.6$ & $36.8 \pm 1.4$ & 5.8 & $\mathrm{~K} 2 \mathrm{~V}$ & $\ldots$ & 8.3 & 1.1 \\
\hline HD 80606 & $09: 22: 37.56$ & $+50: 36: 13.43$ & $58.8 \pm 1.5$ & $13.2 \pm 1.6$ & $58.0 \pm 20.0$ & 7.3 & G5 & $\ldots$ & 9.7 & 1.0 \\
\hline HD 85301 & $09: 52: 16.77$ & $+49: 11: 26.84$ & $-213.7 \pm 1.2$ & $-68.9 \pm 1.3$ & $32.0 \pm 1.0$ & 6.1 & G5 & $\cdots$ & 9.1 & 1.0 \\
\hline HD 88638 & $10: 14: 35.76$ & $+53: 46: 15.51$ & $-270.9 \pm 1.5$ & $67.1 \pm 1.5$ & $38.0 \pm 4.0$ & 6.3 & G5 & $\ldots$ & 9.5 & 1.0 \\
\hline HD 91962 & 10:37:00.02 & $-08: 50: 23.63$ & $-94.1 \pm 0.8$ & $-48.8 \pm 0.8$ & $37.0 \pm 2.0$ & 5.4 & G1V & $\ldots$ & 8.4 & 1.0 \\
\hline HD 92788 & $10: 42: 48.54$ & $-02: 11: 01.38$ & $-11.8 \pm 1.2$ & $-223.8 \pm 1.3$ & $32.0 \pm 1.0$ & 5.7 & G6V & $\ldots$ & 9.7 & 1.1 \\
\hline HD 98553 & 11:20:11.60 & $-19: 34: 40.54$ & $69.1 \pm 1.1$ & $-68.9 \pm 1.2$ & $34.0 \pm 1.0$ & 6.1 & $\mathrm{G} 2 / 3 \mathrm{~V}$ & $\ldots$ & 9.2 & 1.1 \\
\hline HD 99565 & $11: 27: 10.76$ & $-15: 38: 55.05$ & $1.6 \pm 1.1$ & $-197.2 \pm 1.3$ & $35.0 \pm 3.0$ & 5.8 & G8V & $\ldots$ & 9.2 & 0.9 \\
\hline HD 100167 & $11: 31: 53.92$ & $+41: 26: 21.65$ & $-42.7 \pm 1.1$ & $83.5 \pm 1.1$ & $35.0 \pm 1.0$ & 5.8 & F8 & $\ldots$ & 9.3 & 1.1 \\
\hline HD 101959 & $11: 43: 56.62$ & $-29: 44: 51.80$ & $-272.7 \pm 1.6$ & $37.4 \pm 1.3$ & $32.0 \pm 1.0$ & 5.6 & G0V & $\ldots$ & 9.2 & 1.1 \\
\hline HD 102071 & $11: 44: 39.32$ & $-29: 53: 05.46$ & $-71.9 \pm 1.5$ & $49.7 \pm 1.4$ & $30.0 \pm 1.0$ & 6.1 & $\mathrm{~K} 0 \mathrm{~V}$ & $\ldots$ & 9.3 & 0.9 \\
\hline HD 103432 & $11: 54: 32.07$ & $+19: 24: 40.44$ & $-449.9 \pm 1.0$ & $-15.6 \pm 0.8$ & $37.0 \pm 2.0$ & 6.5 & G6V & $\ldots$ & 9.3 & 1.0 \\
\hline HD 105631 & $12: 09: 37.26$ & $+40: 15: 07.62$ & $-314.3 \pm 0.7$ & $-51.3 \pm 0.8$ & $24.0 \pm 1.0$ & 5.6 & G9V & $\ldots$ & 9.2 & 0.9 \\
\hline HD 106156 & $12: 12: 57.52$ & $+10: 02: 15.62$ & $210.5 \pm 1.2$ & $-357.6 \pm 1.1$ & $31.0 \pm 1.0$ & 6.1 & G8V & $\ldots$ & 9.3 & 1.0 \\
\hline HD 106252 & $12: 13: 29.49$ & $+10: 02: 29.96$ & $24.2 \pm 1.1$ & $-280.3 \pm 1.1$ & $37.0 \pm 1.0$ & 5.9 & G0 & $\ldots$ & 9.5 & 1.1 \\
\hline HD 108799 & $12: 30: 04.77$ & $-13: 23: 35.14$ & $-250.5 \pm 2.1$ & $-47.0 \pm 2.2$ & $25.0 \pm 1.0$ & 4.8 & $\mathrm{G} 1 / 2 \mathrm{~V}$ & $\ldots$ & 8.3 & 1.2 \\
\hline HD 108944 & $12: 31: 00.74$ & $+31: 25: 25.84$ & $9.2 \pm 1.1$ & $25.1 \pm 1.2$ & $44.0 \pm 2.0$ & 6.0 & F9V & $\ldots$ & 8.2 & 1.2 \\
\hline HD 112196 & $12: 54: 40.02$ & $+22: 06: 28.65$ & $52.1 \pm 0.9$ & $-33.9 \pm 0.9$ & $34.0 \pm 2.0$ & 5.6 & F8V & $\ldots$ & 7.9 & 1.1 \\
\hline HD 115043 & $13: 13: 37.01$ & $+56: 42: 29.82$ & $112.8 \pm 0.9$ & $-19.5 \pm 1.0$ & $26.0 \pm 0.4$ & 5.3 & G1V & $\ldots$ & 8.7 & 1.1 \\
\hline HD 121320 & $13: 54: 28.20$ & $+20: 38: 30.46$ & $210.1 \pm 1.0$ & $-76.3 \pm 1.0$ & $33.0 \pm 1.0$ & 6.2 & G5V & $\ldots$ & 9.3 & 1.0 \\
\hline HD 122652 & $14: 02: 31.63$ & $+31: 39: 39.09$ & $-94.5 \pm 1.3$ & $8.8 \pm 1.3$ & $37.0 \pm 1.0$ & 5.9 & F8 & $\ldots$ & 9.3 & 1.2 \\
\hline HD 129333 & $14: 39: 00.25$ & $+64: 17: 29.94$ & $-135.9 \pm 1.1$ & $-25.3 \pm 1.2$ & $34.0 \pm 1.0$ & 5.9 & G5V & $\ldots$ & 7.9 & 1.0 \\
\hline HD 133295 & $15: 04: 33.08$ & $-28: 18: 00.65$ & $40.1 \pm 1.4$ & $-51.9 \pm 1.4$ & $34.0 \pm 1.0$ & 5.8 & $\mathrm{G} 0 / 1 \mathrm{~V}$ & $\ldots$ & 8.5 & 1.1 \\
\hline HD 134319 & $15: 05: 49.90$ & $+64: 02: 50.00$ & $-123.3 \pm 1.1$ & $110.1 \pm 1.2$ & $44.0 \pm 1.0$ & 6.8 & G5(V) & $\ldots$ & 7.8 & 1.0 \\
\hline HD 135363 & $15: 07: 56.31$ & $+76: 12: 02.66$ & $-130.5 \pm 1.3$ & $163.7 \pm 1.3$ & $29.0 \pm 1.0$ & 6.2 & G5(V) & $\ldots$ & 7.8 & 0.7 \\
\hline HD 136923 & $15: 22: 46.84$ & $+18: 55: 08.31$ & $-230.9 \pm 1.1$ & $77.2 \pm 1.1$ & $20.0 \pm 0.4$ & 5.3 & G9V & $\ldots$ & 9.3 & 0.9 \\
\hline HD 138004 & $15: 27: 40.36$ & $+42: 52: 52.82$ & $-60.2 \pm 0.8$ & $-259.4 \pm 0.8$ & $32.0 \pm 1.0$ & 5.9 & G2III & $\ldots$ & 9.3 & 1.0 \\
\hline RX J1541.1-2656 & $15: 41: 06.79$ & $-26: 56: 26.33$ & $-15.5 \pm 5.5$ & $-29.7 \pm 1.6$ & $145.0 \pm 40.0$ & 8.9 & G7 & USco & 6.7 & 0.8 \\
\hline HD 142229 & $15: 53: 20.02$ & $+04: 15: 11.51$ & $-24.4 \pm 1.1$ & $9.7 \pm 1.0$ & $41.0 \pm 2.0$ & 6.6 & G5V & 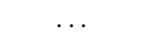 & 8.8 & 1.0 \\
\hline
\end{tabular}


Table 2

(Continued)

\begin{tabular}{|c|c|c|c|c|c|c|c|c|c|c|}
\hline Star & $\begin{array}{c}\alpha \\
(\mathrm{J} 2000.0) \\
\end{array}$ & $\begin{array}{c}\delta \\
(\mathrm{J} 2000.0) \\
\end{array}$ & $\begin{array}{c}\mu_{\alpha} \cos \delta \\
\left(\mathrm{mas} \mathrm{yr}^{-1}\right)\end{array}$ & $\begin{array}{c}\mu_{\delta} \\
\left(\operatorname{mas~yr}^{-1}\right)\end{array}$ & $\begin{array}{c}d \\
(\mathrm{pc})\end{array}$ & $\begin{array}{c}K_{S} \\
(\mathrm{mag}) \\
\end{array}$ & Sp.T. & Association & $\log$ Age $\mathrm{yr}^{-1}$ & $\begin{array}{l}\text { Mass } \\
\left(M_{\odot}\right) \\
\end{array}$ \\
\hline RX J1600.6-2159 & $16: 00: 40.57$ & $-22: 00: 32.24$ & $-14.2 \pm 1.7$ & $-18.8 \pm 1.7$ & $145.0 \pm 40.0$ & 8.4 & G9 & USco & 6.7 & 1.1 \\
\hline PZ99 J160814.7-190833 & 16:08:14.74 & - 19:08:32.77 & $-32.0 \pm 7.3$ & $-4.1 \pm 7.7$ & $145.0 \pm 40.0$ & 8.4 & $\mathrm{~K} 2$ & USco & 6.7 & 1.1 \\
\hline HD 145229 & 16:09:26.63 & $+11: 34: 28.25$ & $-99.5 \pm 0.9$ & $102.9 \pm 1.2$ & $33.0 \pm 1.0$ & 6.0 & G0 & & 8.8 & 1.1 \\
\hline PZ99 J161329.3-231106 & $16: 13: 29.29$ & $-23: 11: 07.56$ & $-12.4 \pm 2.0$ & $-30.8 \pm 2.5$ & $145.0 \pm 40.0$ & 8.5 & $\mathrm{~K} 1$ & USco & 6.7 & 1.0 \\
\hline HD 150706 & $16: 31: 17.63$ & $+79: 47: 23.15$ & $95.1 \pm 0.8$ & $-89.2 \pm 0.8$ & $27.0 \pm 0.4$ & 5.6 & G3(V) & $\ldots$ & 8.8 & 1.1 \\
\hline HD 150554 & $16: 40: 56.45$ & $+21: 56: 53.24$ & $-93.6 \pm 1.0$ & $5.2 \pm 1.0$ & $45.0 \pm 2.0$ & 6.3 & F8 & $\ldots$ & 9.4 & 1.1 \\
\hline HD 152555 & 16:54:08.15 & $-04: 20: 24.89$ & $-37.2 \pm 1.2$ & $-114.3 \pm 1.3$ & $48.0 \pm 3.0$ & 6.4 & F8/G0V & $\ldots$ & 8.1 & 1.1 \\
\hline HD 153458 & 17:00:01.66 & $-07: 31: 53.93$ & $97.3 \pm 1.3$ & $-20.2 \pm 1.0$ & $44.0 \pm 2.0$ & 6.4 & G5V & $\ldots$ & 9.2 & 1.1 \\
\hline HD 154417 & $17: 05: 16.83$ & $+00: 42: 09.18$ & $-16.8 \pm 0.9$ & $-334.8 \pm 0.9$ & $20.0 \pm 0.4$ & 4.6 & F9V & $\ldots$ & 9.1 & 1.1 \\
\hline HD 155902 & $17: 11: 08.43$ & $+56: 39: 33.10$ & $-2.1 \pm 1.2$ & $-68.6 \pm 1.3$ & $28.0 \pm 1.0$ & 5.2 & G5 & $\ldots$ & 9.3 & 0.9 \\
\hline HD 157664 & $17: 18: 58.47$ & $+68: 52: 40.61$ & $32.0 \pm 1.1$ & $5.5 \pm 1.1$ & $84.0 \pm 5.0$ & 6.7 & G0 & $\ldots$ & 9.6 & 1.2 \\
\hline HD 159222 & 17:32:00.99 & $+34: 16: 15.97$ & $-240.0 \pm 1.3$ & $63.3 \pm 1.5$ & $24.0 \pm 0.3$ & 5.0 & G1V & $\ldots$ & 9.4 & 1.1 \\
\hline HD 161897 & 17:41:06.70 & $+72: 25: 13.41$ & $-121.8 \pm 1.4$ & $294.6 \pm 1.4$ & $29.0 \pm 1.0$ & 5.9 & K0 & $\ldots$ & 9.3 & 1.0 \\
\hline HD 166435 & 18:09:21.39 & $+29: 57: 06.08$ & $71.4 \pm 1.1$ & $59.4 \pm 1.1$ & $25.0 \pm 0.4$ & 5.3 & G1IV & $\ldots$ & 9.5 & 1.1 \\
\hline HD 167389 & 18:13:07.22 & $+41: 28: 31.33$ & $51.4 \pm 0.8$ & $-128.1 \pm 0.8$ & $33.0 \pm 1.0$ & 5.9 & $\mathrm{~F} 8(\mathrm{~V})$ & $\ldots$ & 9.3 & 1.1 \\
\hline HD 175742 & $18: 55: 53.14$ & $+23: 33: 26.40$ & $130.8 \pm 0.8$ & $-283.1 \pm 0.6$ & $21.4 \pm 0.5$ & 6.1 & K0 & $\ldots$ & 9.5 & 0.8 \\
\hline HD 179949 & $19: 15: 33.23$ & $-24: 10: 45.61$ & $116.6 \pm 0.9$ & $-101.7 \pm 0.9$ & $27.0 \pm 1.0$ & 4.9 & F8V & $\ldots$ & 9.3 & 1.2 \\
\hline HD 187897 & 19:52:09.38 & $+07: 27: 36.10$ & $133.6 \pm 1.7$ & $66.5 \pm 1.6$ & $33.0 \pm 1.0$ & 5.7 & G5 & $\ldots$ & 9.2 & 1.1 \\
\hline HD 190228 & 20:03:00.77 & $+28: 18: 24.46$ & $108.0 \pm 1.1$ & $-72.4 \pm 1.1$ & $62.0 \pm 3.0$ & 5.4 & G5IV & $\ldots$ & 10.0 & 1.4 \\
\hline HD 193216 & 20:16:54.53 & $+50: 16: 43.55$ & $-221.8 \pm 1.1$ & $-221.2 \pm 1.1$ & $31.0 \pm 2.0$ & 6.4 & G5 & $\ldots$ & 9.3 & 0.9 \\
\hline HD 193017 & 20:18:10.00 & $-04: 43: 43.23$ & $-26.8 \pm 1.0$ & $-21.9 \pm 1.2$ & $37.0 \pm 1.0$ & 6.0 & F6V & $\ldots$ & 9.2 & 1.1 \\
\hline HD 195034 & 20:28:11.81 & $+22: 07: 44.34$ & $-23.3 \pm 1.1$ & $-243.4 \pm 1.0$ & $28.0 \pm 1.0$ & 5.6 & G5 & $\ldots$ & 9.3 & 1.1 \\
\hline HD 199143 & $20: 55: 47.68$ & $-17: 06: 51.02$ & $62.2 \pm 1.5$ & $-65.4 \pm 1.3$ & $48.0 \pm 2.0$ & 5.8 & F8V & $\ldots$ & 7.2 & 1.3 \\
\hline HD 199598 & 20:57:39.68 & $+26: 24: 18.40$ & $266.6 \pm 1.1$ & $92.4 \pm 1.1$ & $33.0 \pm 1.0$ & 5.5 & G0V & $\ldots$ & 9.2 & 1.2 \\
\hline HD 201219 & 21:07:56.53 & $+07: 25: 58.47$ & $189.0 \pm 1.9$ & $-11.5 \pm 1.8$ & $36.0 \pm 2.0$ & 6.4 & G5 & $\ldots$ & 9.0 & 1.0 \\
\hline HD 202108 & $21: 12: 57.63$ & $+30: 48: 34.25$ & $-20.1 \pm 1.6$ & $108.4 \pm 1.6$ & $27.0 \pm 1.0$ & 5.8 & G3V & $\ldots$ & 9.2 & 1.0 \\
\hline HD 201989 & 21:14:01.80 & $-29: 39: 48.85$ & $231.6 \pm 1.2$ & $-38.7 \pm 1.2$ & $30.0 \pm 1.0$ & 5.7 & $\mathrm{G} 3 / 5 \mathrm{~V}$ & $\ldots$ & 9.0 & 1.0 \\
\hline HD 204277 & 21:27:06.61 & $+16: 07: 26.85$ & $-80.1 \pm 1.1$ & $-96.5 \pm 1.1$ & $34.0 \pm 1.0$ & 5.4 & F8V & $\ldots$ & 8.7 & 1.2 \\
\hline HIP 106335 & 21:32:11.69 & $+00: 13: 17.90$ & $415.3 \pm 2.5$ & $28.0 \pm 1.4$ & $49.4 \pm 4.9$ & 7.1 & $\mathrm{~K} 3 \mathrm{Ve}+$ & $\ldots$ & 8.7 & 0.9 \\
\hline HD 205905 & 21:39:10.14 & $-27: 18: 23.59$ & $386.9 \pm 1.7$ & $-84.8 \pm 1.4$ & $26.0 \pm 1.0$ & 5.3 & $\mathrm{G} 2 \mathrm{~V}$ & $\ldots$ & 9.1 & 1.1 \\
\hline HD 206374 & 21:41:06.19 & $+26: 45: 02.25$ & $343.4 \pm 1.0$ & $-90.0 \pm 1.0$ & $27.0 \pm 1.0$ & 5.8 & G6.5V & .. & 9.2 & 1.0 \\
\hline HD 212291 & 22:23:09.17 & $+09: 27: 39.95$ & $304.6 \pm 1.3$ & $33.6 \pm 1.3$ & $32.0 \pm 1.0$ & 6.3 & G5 & $\ldots$ & 9.3 & 1.0 \\
\hline HD 216275 & $22: 50: 46.34$ & $+52: 03: 41.21$ & $144.4 \pm 1.0$ & $170.0 \pm 1.2$ & $31.0 \pm 1.0$ & 5.8 & G0 & $\ldots$ & 9.3 & 1.1 \\
\hline RX J2312.0+2245 & $23: 12: 04.52$ & $+22: 45: 26.28$ & $23.7 \pm 0.9$ & $-16.5 \pm 0.6$ & $150.0 \pm 75.0$ & 8.3 & G3 & $\ldots$ & 8.7 & 1.4 \\
\hline RX J2313.0+2345 & 23:13:01.24 & $+23: 45: 29.64$ & $12.4 \pm 0.9$ & $-11.4 \pm 0.6$ & $150.0 \pm 75.0$ & 8.6 & F8 & $\ldots$ & 7.0 & 1.1 \\
\hline HD 221613 & $23: 33: 24.06$ & $+42: 50: 47.88$ & $243.2 \pm 1.0$ & $177.1 \pm 1.0$ & $33.0 \pm 1.0$ & 5.5 & G0 & $\ldots$ & 9.3 & 1.0 \\
\hline
\end{tabular}

Note. Column names and target catalog identifiers are as described in Table 1.

(This table is also available in a machine-readable form in the online journal.)

Table 3

Median Sample Statistics

\begin{tabular}{|c|c|c|c|c|c|c|}
\hline \multirow[t]{2}{*}{ Sample } & \multicolumn{2}{|c|}{ 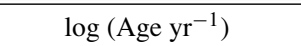 } & \multicolumn{2}{|c|}{ Distance (pc) } & \multicolumn{2}{|c|}{ Spectral Type } \\
\hline & Range & Median & Range & Median & Range & Median \\
\hline Deep & $6.6-8.7$ & 8.0 & $10-190$ & 50 & F5-K5 & G5 \\
\hline Shallow & $6.6-10.0$ & 8.8 & $11-190$ & 45 & F5-K5 & G7 \\
\hline Complete & $6.6-10.0$ & 8.3 & $10-190$ & 46 & F5-K5 & G5 \\
\hline
\end{tabular}

up was obtained during 2006 and 2007 in a few individual cases.

We opted to conduct the entire survey in the $K_{S}$ band to take advantage of the much better $\mathrm{AO}$ performance at $>2 \mu \mathrm{m}$. Although cool T-type brown dwarfs $\left(T_{\text {eff }} \lesssim 1400 \mathrm{~K}\right.$; Golimowski et al. 2004; Vrba et al. 2004) are faintest at the $K$ band in the NIR, warmer (potentially younger) L-type brown dwarfs are brightest at $K$. Thus, given superior imaging contrast and the relative youth of our deep sample, the $2 \mu \mathrm{m}$ region was seen as the best choice for optimizing sensitivity to close-in young substellar companions.

The majority of the science targets were observed first at Palomar. Only seven of the targets (all belonging to the deep sample) were observed initially and only at Keck. The Palomar campaign was conducted with the Palomar Adaptive Optics (PALAO) system (Troy et al. 2000) and the Palomar HighAngular Resolution Observer (PHARO) NIR camera (Hayward et al. 2001) in its 25 mas pix $^{-1}$ mode, providing a $25^{\prime \prime} .6 \times 25^{\prime \prime} .6$ FOV. At Keck, we used the facility AO system (Wizinowich et al. 2000) on Keck II and the NIRC2 NIR camera in its 40 mas pix $^{-1}$ mode, offering an FOV of $40^{\prime \prime} 6 \times 40^{\prime} \cdot 6$. To improve overall sensitivity and contrast, the 100 targets in the deep sample were observed coronagraphically with the opaque 0 .97 diameter occulting spot in PHARO and the partiallytransmissive 1".0 diameter occulting spot in NIRC2. All of the sample stars were sufficiently bright to allow use of the 


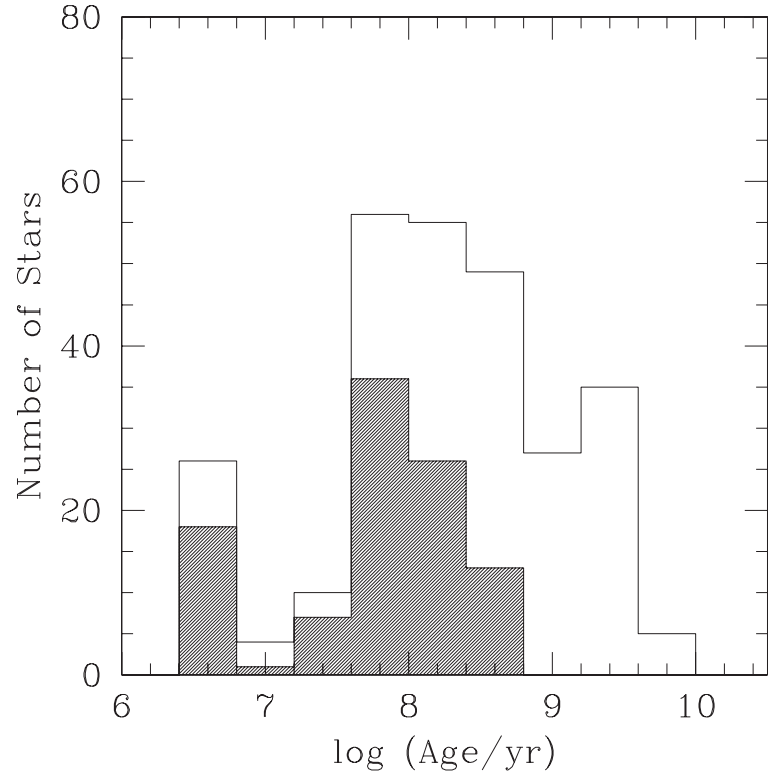

Figure 2. Age distributions of the complete survey sample (nonshaded histogram) and of the deep subsample (shaded histogram).

AO systems in the natural guide star (NGS) mode, that is, to have the wavefront sensing performed on the primaries themselves.

\subsubsection{First-Epoch Observations at Palomar}

We spent 24 minutes of net exposure time per target during the first epoch at Palomar, attaining an imaging depth of $K_{S} \approx$ 19.7 mag on average for stars in the deep sample. The 24 minutes of exposure were divided into four sets of 6 minutes taken at different orientations of the telescope Cassegrain ring (CR) rotator. The $6 \mathrm{~min}$ of net exposure at each CR rotator angle consisted of two sets of three 1 minute on-target exposures, with three 1 minute sky exposures in between. The purpose of the $\mathrm{CR}$ rotation approach was to improve the quality of point-spread function (PSF) subtractions for data taken with an equatorialmount telescope (Palomar), in a manner similar to that attained with angular differential imaging (ADI) on altitude-azimuthmounted telescopes (Marois et al. 2006). Stacking images taken at different $\mathrm{CR}$ angles also averages out detector and sky noise, much like mosaicking dithered images. Unfortunately, in addition to being less efficient, the CR rotation approach was later found to also produce notable smearing of the PSF in the coadded derotated images at $\gtrsim 5^{\prime \prime}$ from the star, leading to degradation both in imaging depth and in astrometric precision (Metchev 2006). We have since demonstrated that judicious matching of nearby science targets to use as PSFs for one another - a suitable approach for surveys of targetrich young stellar associations - enhances the contrast attainable with PALAO by $0.5-1.0$ mag over that reported here, without incurring the overhead of CR rotations (Tanner et al. 2007).

We used two different undersized Lyot stops to block the secondary obscuration and the secondary mirror support structure at Palomar: the "medium" and the "big" crosses, obscuring $40 \%$ and $76 \%$ of the total telescope aperture, respectively (Hayward et al. 2001). The use of an appropriately-sized Lyot stop was expected to noticeably improve the dynamic range achievable in high-order AO coronagraphy by suppressing light diffracted by the edge of the coronagraph (Sivaramakrishnan et al. 2001). Early experiments with the PALAO/PHARO system by Oppenheimer et al. (2000) had suggested that the big cross provided the best contrast in single exposures of up to several seconds, outperforming the medium and "standard" (no undersizing) Lyot masks by up to 0.5 mag between 0 .'5 and 2".0 from bright stars. However, our experience from observing each star in multiple longer exposures was that the less oversized Lyot stops allowed better real-time monitoring of the star-coronagraph alignment and more accurate postprocessing image registration and astrometry. With the medium and the standard Lyot stops, the position of the star behind the coronagraph could be monitored by the location of a Poisson-like spot within the dark area of the coronagraph - the result of constructive interference of high spatial frequency light pushed by the coronagraph to the periphery of the Lyot plane. The big Lyot stop likely shutters incoming starlight too aggressively to allow the formation of a sufficiently bright Poisson spot. Because image registration of multiple exposures was crucial for obtaining greater overall exposure depth, we stopped using the big cross after 2002 March. Given the adopted technique of rotating the CR to four mutually orthogonal orientations during the imaging of each star, the medium cross provided the best compromise between registration ability for the final images and consistency with which it would obscure the telescope secondary mirror support at each
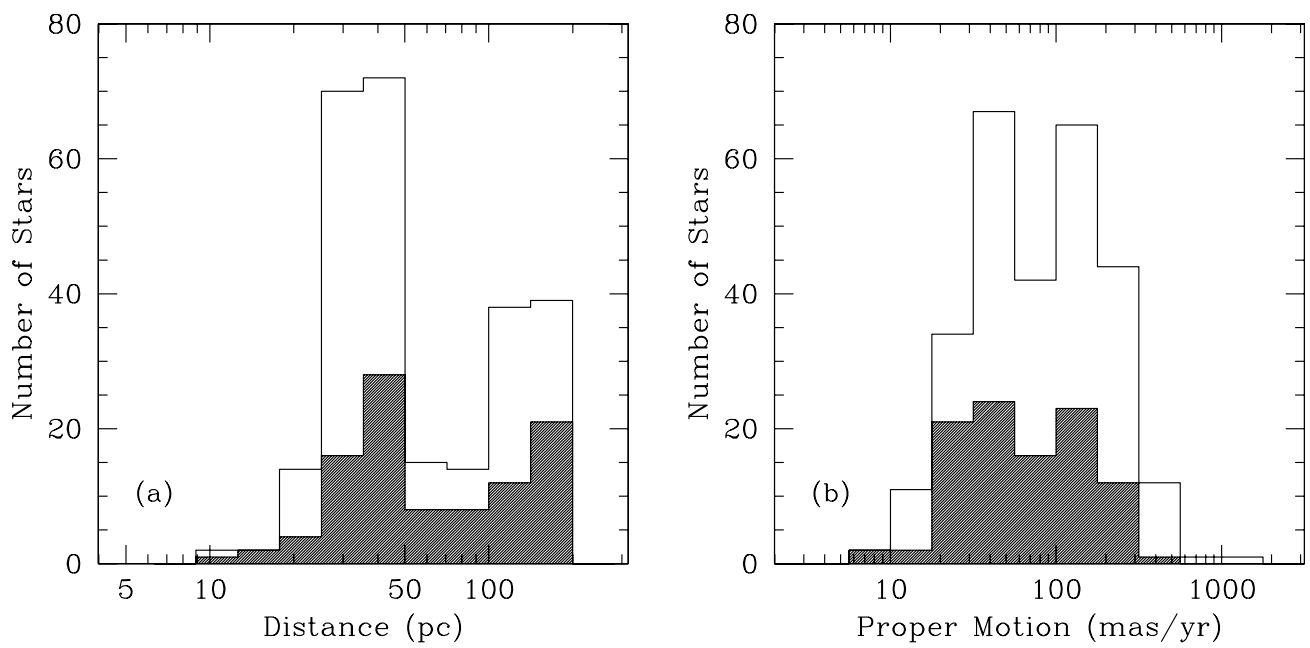

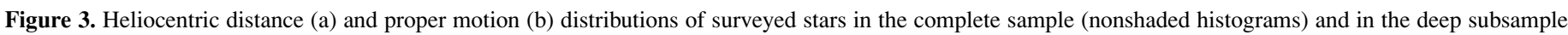
(shaded histograms). 
CR orientation. At the end of our survey, only seven of the 100 stars in the deep sample had their deepest observations obtained with the big Lyot cross setup.

In addition to the long coronagraphic $K_{S}$-band exposures, we also observed each deep sample target in short (1.4-10 s) unocculted exposures. These were taken to check for stellar multiplicity within the 0 '.5 coronagraph radius and to allow relative photometric calibration of the deep occulted exposures. The short exposures were obtained at $J, H$, and $K_{S}$ bands, using a $1 \%$ neutral density (ND) filter to prevent saturation whenever necessary. For these observations we used a five-point dither pattern, as is standard for IR imaging.

The 166 targets in the shallow sample were observed only in short dithered $J H K_{S}$ exposures at Palomar to check for stellar multiplicity. The imaging depth of the shallow survey greatly varied from star to star, depending on whether the ND filter was used or not, and was generally in the $12 \mathrm{mag}<K_{S}<17$ mag range.

\subsubsection{Follow-up Observations}

After an examination of the initial Palomar images, target stars that contained other objects in the same image-candidate companions - were followed up with additional imaging at later epochs to test for common proper motion between the candidate companions and the host stars. Upon establishing common proper motion, candidate companions were spectroscopically observed to confirm their physical association with the primary.

The imaging and spectroscopic follow-up was performed at both Palomar and Keck, respectively. Imaging at Keck was done in $6 \times 1$ minute coronagraphic integrations per target, with an additional $3 \times 1$ minute spent on sky. We used the "inscribed circle" NIRC2 pupil mask (90.7\% throughput) to occult the telescope mirror outer edge (none of the available NIRC2 pupil masks occult the Keck segment edges and the secondary support structure). In most cases, the 6 minute long exposures at Keck were $\approx 0.5 \mathrm{mag}$ deeper $\left(K_{S} \approx 20.2 \mathrm{mag}\right)$ than the 24 minute Palomar exposures, and occasionally revealed new candidate companions. Nearly half (48/100) of our deep sample stars were observed at Keck in addition to Palomar, including the seven targets observed only at Keck. Because of the marginal difference in the depths of the Keck and Palomar components of the deep survey, and for the sake of preserving the integrity of our well-defined 100 star deep sample, we analyze the Palomar and Keck AO campaigns together as a single survey.

We obtained NIR spectra of several bona fide and candidate companions for the purposes of further confirmation of their physical association and characterization of their photospheres. The spectroscopic observations and data reduction were described in Metchev \& Hillenbrand (2004, 2006). Here, we present spectroscopy of only one additional companion candidate to ScoPMS 214. The observations and data reduction for that are briefly described in Section 6.3.1.

\subsection{Imaging Data Reduction}

The imaging data reduction procedure for the survey, including flat fielding, sky-subtraction, bad-pixel correction, image registration, and image stacking, was detailed in Metchev \& Hillenbrand (2004). We have since augmented the procedure to include a correction for the nonlinear flux response of the PHARO and NIRC2 detectors. NIR detectors often have a nonlinear response even at small flux levels, which is important to take into account when seeking accurate photometry. We measured the nonlinearity of the PHARO and NIRC2 detectors from series of variable-length exposures of the brightly illuminated telescope dome interiors, interspersed with multiple dark frames to mitigate charge persistence effects. The response of the PHARO camera, which employs an $\mathrm{HgCdTe}$ detector that does not support multiple nondestructive reads, was found to be $>1 \%$ nonlinear beyond 10,000 counts pix $^{-1}$ and $>5 \%$ nonlinear beyond 45,000 counts pix $^{-1}$. The InSb detector on NIRC2, which supports nondestructive read-outs, was found to be $>1 \%$ nonlinear beyond 3000 counts pix $^{-1}$ per read and $>5 \%$ nonlinear beyond 7000 counts pix $^{-1}$ per read. We created custom IDL routines ${ }^{5}$ to linearize the PHARO and NIRC2 flux responses. The linearization was applied to all images before any other data reduction steps.

To enhance our ability to detect faint candidate companions in the deep coronagraphic exposures we attempted various methods of PSF removal, including: (1) subtracting a mediancombined PSF of the star formed from the individual images taken at all four CR angles at Palomar, (2) subtraction of a $180^{\circ}$ rotated version of the image centered on the star from itself, (3) high-pass filtering by subtracting a Gaussian-smoothed (Gaussian full width of half-maximum $(\mathrm{FWHM})=1-3 \times$ PSF FWHM) version of the image from itself, and (4) simple subtraction of an azimuthally medianed radial profile. We found that (1-3) gave comparable results, while (4) did not perform as well as the rest because of the four-cornered or six-spoked symmetry of the PALAO or Keck AO PSFs. Even though (3) is arguably the most widely used method for PSF subtraction when separate PSF observations are not available and when the observations are not taken using ADI, we found that because of the central symmetry of the brightest AO speckles (Boccaletti et al. 2002; Bloemhof 2003) method (2) worked almost as well. Method (2) also did not alter the photon statistics of the PSFsubtracted image in the spatially correlated manner incurred by Gaussian smoothing. Therefore, for PSF subtraction, we relied on method (2) the most.

\subsection{Precision Astrometry}

Multiepoch astrometry is essential for demonstrating physical association of bound pairs. This is the principal method employed for candidate companion confirmation here. Below we discuss the calibration steps that we undertook to ensure self-consistent astrometric measurements throughout our campaign.

We calibrated our astrometry by obtaining repeated measurements of the positions of well-known visual binaries at each observing epoch. We selected binary stars with well-known ephemeris from the Sixth Orbit Catalog (Hartkopf et al. 2001, Hartkopf \& Mason 2003), combining binaries with grade 1 (accurately determined, short-period) and grade 4 (less accurately known, longer-period) orbital solutions, as recommended for astrometric calibration by (Hartkopf \& Mason 2003). Despite the lower quality of the orbital solutions for the grade 4 binaries, their periods are generally much longer, so that their motions are predicted with sufficient accuracy for many years into the future. The selected calibration binaries and their orbital parameters are given in Table 4.

The above astrometric calibration was adequate for detecting astrometric signals $\delta \rho / \rho \gtrsim 1 \%$ with PHARO. Such accuracy

\footnotetext{
5 The PHARO and NIRC2 detector linearization routines are available at http://www.astro.caltech.edu/palomar/200inch/palao/Pharo/pharo.html and http://www.astro.sunysb.edu/metchev/ao.html, respectively.
} 
Table 4

Calibration Binaries and Assumed Parameters of Their Astrometric Orbits

\begin{tabular}{|c|c|c|c|c|c|c|c|c|c|}
\hline $\begin{array}{l}\text { Binary } \\
\text { (WDS) }\end{array}$ & $\begin{array}{c}a \\
(\operatorname{arcsec})\end{array}$ & $\begin{array}{c}P \\
\text { (years) }\end{array}$ & $\begin{array}{c}T_{0} \\
\text { (year) }\end{array}$ & $e$ & $\begin{array}{c}i \\
\text { (degrees) }\end{array}$ & $\begin{array}{c}\Omega \\
\text { (degrees) }\end{array}$ & $\begin{array}{c}\omega \\
\text { (degrees) }\end{array}$ & Grade & Ref. \\
\hline $09006+4147$ & $0.6472 \pm 0.0010$ & $21.776 \pm 0.017$ & $1993.725 \pm 0.023$ & $0.1507 \pm 0.0008$ & $131.26 \pm 0.13$ & $204.39 \pm 0.19$ & $32.52 \pm 0.36$ & 1 & 1 \\
\hline $16147+3352$ & 5.927 & 888.989 & 1826.949 & 0.7605 & 31.795 & 16.889 & 72.201 & 4 & 2 \\
\hline $18055+0230$ & $4.5540 \pm 0.0052$ & $88.38 \pm 0.02$ & $1895.94 \pm 0.02$ & $0.4992 \pm 0.0004$ & $121.16 \pm 0.08$ & $302.12 \pm 0.10$ & $14.0 \pm 0.1$ & 1 & 3 \\
\hline $20467+1607$ & 10.22 & 3249 & 2305 & 0.88 & 148.78 & 88.06 & 331.16 & 4 & 4 \\
\hline
\end{tabular}

Note. Explanation of orbital parameters: $a$-semimajor axis; $P$-period; $T_{0}$ —epoch of periastron; $e$ - eccentricity; $i$-inclination; $\Omega$-longitude of periastron; $\omega$-longitude of the ascending node.

References. (1) Hartkopf et al. (1996); (2) Scardia (1979); (3) Pourbaix (2000); (4) Hale (1994).

allowed the confirmation of the first brown dwarf companion in our survey, HD 49197B (Metchev \& Hillenbrand 2004). However, that initial calibration assumed that the pixel scale and field orientation over the entire PHARO detector were well determined from measurements taken near the center of the array, ignoring possible image distortion in the focal plane. In reality, the PHARO beam is known to be distorted (Hayward et al. 2001). Accurate characterization of this distortion was necessary before considering the results from our complete survey, which focused on stars with small proper motions (10-

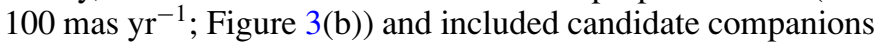
over the entire $25^{\prime \prime} .6 \times 25^{\prime \prime} .6$ PHARO FOV.

We arrived at an improved astrometric calibration of the PHARO 25 mas pix $^{-1}$ camera in Metchev (2006, Chapter 4), where we determined the full extent of the focal plane distortion over the entire array and solved for its dependence on telescope hour angle, declination, and orientation of the $\mathrm{CR}$ rotator. For that calibration, we used a custom-made astrometric mask with pinholes distributed on a rectangular grid that we inserted in the telescope beam path at the Cassegrain focus. From exposures taken with the mask in place, we measured the variations in the spacing among the pinhole images with changes in the instrument gravity vector. We found that the PHARO pixel scale varied by up to $\delta \rho / \rho=0.9 \%$ from the center to the corner of the array in the 25 mas pix $^{-1}$ camera. After fitting twodimensional polynomials to the distortion, we calibrated the variation to within $0.15 \%$ over the entire chip. The polynomial fits to the focal plane distortion on the PHARO 25 mas pix ${ }^{-1}$ camera and its dependence on telescope pointing are given by Equations (4.1)-(4.4) and (4.7)-(4.11), respectively, and Tables 4.4-4.5 in Metchev (2006). An IDL program that corrects for the distortion at an arbitrary coordinate on the PHARO 25 mas pix $^{-1}$ camera is available at the PHARO instrument Web site, http://www.astro.caltech.edu/palomar/200inch/palao/Pharo/ pharo.html. ${ }^{6}$

A similar astrometric calibration has already been performed for all three NIRC2 cameras during the pre-ship testing of the instrument (Thompson et al. 2001). Because NIRC2 sits on the Keck II Nasmyth platform and thus has a constant gravity vector, the distortion of the camera pixel scales does not change with telescope pointing. We implemented the existing astrometric calibration of the NIRC2 cameras in the analysis of our Keck AO imaging data. ${ }^{7}$

\footnotetext{
6 The PALAO/PHARO astrometric calibration was performed in 2005 March. The optics on the PALAO system have since been realigned to accommodate recent and future science instrument upgrades. The astrometric calibration presented here is not applicable to PALAO data taken since 2007.

7 A more precise astrometric calibration of the NIRC2 cameras has since been obtained by Cameron et al. (2009).
}

\subsection{Photometry}

We used 1-2 × PSF FWHM diameter apertures for object photometry, with the smaller apertures used on fainter sources for higher signal-to-noise measurements. The diffractionlimited FWHM of the $K_{S}$-band PSF of PALAO was consistently $\approx 0$ '. 1 , while for the Keck AO system, it was $\approx 0$ '.05. The local background was measured around each object in an annulus with a wide-enough inner radius so that the halo of the point source did not affect the background measurement. The inner radius was as small as $1.5 \times$ PSF FWHM for faint sources embedded in the halos of bright stars, or as large as 25-30 $\times$ PSF FWHM for the target primaries. The variations in the sizes of the apertures and of the background annuli resulted in photometric uncertainties on the order of $0.10-0.30 \mathrm{mag}$. Uncertainties of $\geqslant 0.5 \mathrm{mag}$ were found in a few isolated cases involving very faint point sources and/or point sources near the edges of the FOV, where the PSF was noticeably distorted by anisoplanatism and circular apertures did not produce accurate photometry.

PSF fitting, rather than aperture photometry, was used to measure the fluxes of closely-separated point sources. The photometric uncertainties in such cases were generally $\leqslant 0.20 \mathrm{mag}$.

For absolute calibration, we relied on the Two Micron All Sky Survey (2MASS) fluxes of the primaries. Photometric measurements were always obtained relative to the fluxes of the target primaries, as measured from the unocculted, short exposures, often taken with the PHARO ND $1 \%$ filter in place. We calibrated the NIR extinction of the ND filter from photometric measurements of three program stars on images taken with and without the filter in place. Images with Keck/NIRC2 were obtained only in coronagraphic mode, predominantly using the $1^{\prime \prime}$ diameter spot, although the $2^{\prime \prime}$ diameter spot was used during 2003 May 16-18. Unlike the PHARO coronagraphic spots, the NIRC2 spots are transmissive, offering the possibility to obtain relative photometry with respect to the primary. A measurement of the throughput of the $2^{\prime \prime}$ spot was given in Metchev \& Hillenbrand (2004). Subsequent observations showed that such measurements were dependent on the quality of the AO correction, possibly because of the amount of additive background caused by light from the stellar halo diffracted within the area of the coronagraph. Thus, approximate relative photometry with the NIRC2 coronagraph is likely feasible only with good AO correction (usually at $H$ or $K$ bands), when the amount of scattered ("spill-over") light within the area of the coronagraph is minimized. Table 5 lists the measured NIR extinction in magnitudes for the PHARO ND $1 \%$ filter and for the $1^{\prime \prime}$ and $2^{\prime \prime}$ NIRC2 coronagraphs. The large apparent difference in the $J$-band transmissivity of the two NIRC2 coronagraphic spots is a probable effect of spill-over 
Table 5

Extinction Due to PHARO and NIRC2 Optics

\begin{tabular}{lccc}
\hline \hline Transmissive Optic & $\begin{array}{c}\Delta J \\
(\mathrm{mag})\end{array}$ & $\begin{array}{c}\Delta H \\
(\mathrm{mag})\end{array}$ & $\begin{array}{c}\Delta K_{S} \\
(\mathrm{mag})\end{array}$ \\
\hline PHARO ND 1\% filter & $4.753 \pm 0.039$ & $4.424 \pm 0.033$ & $4.197 \pm 0.024$ \\
NIRC2 1" coronagraph & $8.36 \pm 0.28$ & $7.78 \pm 0.15$ & $7.10 \pm 0.17$ \\
NIRC2 2" coronagraph & $9.26 \pm 0.09$ & $7.79 \pm 0.22$ & $7.07 \pm 0.22$ \\
\hline
\end{tabular}

(more significant for the smaller spot), aggravated by poorer AO performance at $J$.

\section{OBJECT DETECTION AND DETECTION LIMITS}

\subsection{Object Detection}

Object detection is a straightforward matter to automate in point-source-rich images, where the PSF is radially symmetric, approximately constant in time, and has a well-characterized dependence on image location. Unfortunately, none of these qualifications describe the sparsely populated high-contrast images in our deep survey, in which the main (and frequently only) point source is occulted by the coronagraph. In addition, automated source finding in $\mathrm{AO}$ images of bright stars is hindered by large numbers of speckles. Speckles are individual images of the star that form from uncorrected and/or induced (by the telescope optics) aberrations in the wavefront, and appear indistinguishable from point sources to automated detection routines. As a result, even though certain source detection algorithms have been developed (StarFinder; Diolaiti et al. 2000), or adapted (DAOPHOT II, IDAC; Stetson 1992, Jefferies \& Christou 1993), for diffraction-limited image restoration, they did not produce satisfactory results on our images. Our experiments with DAOPHOT, WAVDETECT (Freeman et al. 2002), and StarFinder produced large numbers of spurious detections, the vast majority of which could be identified with speckles around the coronagraph. If the signal-to-noise threshold in the source-finding algorithms was adjusted to a correspondingly higher level, the algorithms would miss bona fide point sources far from the central star. The performance of the automated algorithms did not change whether we used various methods of PSF subtraction (Section 3.2) or not. Similar experiences and conclusions were drawn independently by Carson et al. (2005), who also used the PALAO/PHARO system for their substellar companion search. Therefore, after some experimentation, and despite an understanding that automated source detection has the potential to offer greater repeatability and conceptual clarity, we abandoned the approach.

Instead, we opted for visual point-source identification, which, barring subjective factors, produces superior results compared to automated detection. We carefully inspected all of the final coronagraphic images for candidate companions. The visual inspection was repeated multiple times during the steps of image reduction, photometry, and astrometry to reduce the effect of subjective factors to a minimum.

The high-contrast imaging literature abounds with examples where the authors have resorted to by-eye identification of candidate companions (e.g., Tokovinin et al. 1999; Brandner et al. 2000; Schroeder et al. 2000; Luhman \& Jayawardhana 2002; McCarthy \& Zuckerman 2004; Masciadri et al. 2005; Luhman et al. 2005; Lafrenière et al. 2007). A notable exception is the study of Lowrance et al. (2005), who applied a rigorous custommade automated detection scheme to their HST/NICMOS data. However, the Lowrance et al. (2005) survey benefited from the well-behaved PSF of space-borne $H S T$ imaging. In a separate instance, Shatsky \& Tokovinin (2002) used DAOPHOT II for their noncoronagraphic AO data. Still, they did not discuss an application of the approach to their set of coronagraphic data, which are likely to be speckle-dominated.

\subsection{Determination of Detection Limits}

We quantified our ability to visually detect faint objects by introducing artificial point sources in the Palomar and Keck $K_{S}$ images of one of our targets, HD 172649, for which data were taken under good observing conditions with Strehl ratios of $\approx 50 \%$. The method was first described in Metchev et al. (2003) and developed more fully in Metchev (2006). We summarize it here briefly.

We introduced 1000-5000 artificial point sources of constant brightness at random locations over the entire $25^{\prime \prime} 6 \times 25^{\prime \prime} 6$ area of the image and counted the number that were retrievable by eye in $0^{\prime \prime} .25-1^{\prime \prime} .0$ wide concentric annuli centered on the star. We recorded both the minimum point-source $K_{S}$ magnitude, at which $100 \%$ of the artificial point sources were visible at the given angular separation, and the maximum $K_{S}$ magnitude, at which only a few artificial point sources were visible. We took the mean of the $K_{S}$ magnitude range as the representative limiting magnitude at the given separation. We repeated the experiment for a range of artificial star magnitudes, at steps of $0.5 \mathrm{mag}$, on both the coronagraphic and the noncoronagraphic images of HD 172649. The PSF for artificial stars in the coronagraphic image was obtained from a fit to the brightest field object $\left(\Delta K_{S}=6.4 \mathrm{mag}\right)$, whereas in the unocculted image, the PSF was obtained from a fit to HD 172649 itself.

The inferred detection limits based on the artificial pointsource experiments are shown in Figure 4. We see that the 6 minute long Keck AO coronagraphic images offered 0.51.5 mag higher contrast and up to 0.5 mag greater depth than the 24 minute PALAO images. The greatest difference in contrast is in the $1^{\prime \prime} .0-1 . .5$ angular separation range, where the presence of waffle-mode distortion in the PALAO PSF limits the attainable contrast.

For the purpose of estimating the completeness of our survey, it was important to determine sensitivity limits on a per-star basis. However, the above approach was too tedious to apply to all observations. Instead, we employed a simpler strategy based on the rms scatter of the pixel counts in the radial profile of each sample star. To match the approximate spatial correlation scale in the PALAO and Keck AO images, we normalized the rms scatter to an aperture with radius equal to the $0^{\prime \prime} 10 \mathrm{FWHM}$ of the PALAO PSF. That is, we multiplied the rms profile by the square root of the number $N$ of pixels in the photometry aperture; $N=50.3$ pix for PALAO/PHARO with the 25 mas pix $^{-1}$ camera and $N=20.0$ pix for Keck AO/NIRC2 with the wide (40 mas pix $^{-1}$ ) camera. This procedure imposed a more stringent requirement on the significance of the detection of a candidate point source by raising the effective multiple of the pixel-to-pixel rms scatter used as a threshold by a factor of 4.5-7.1. We show 


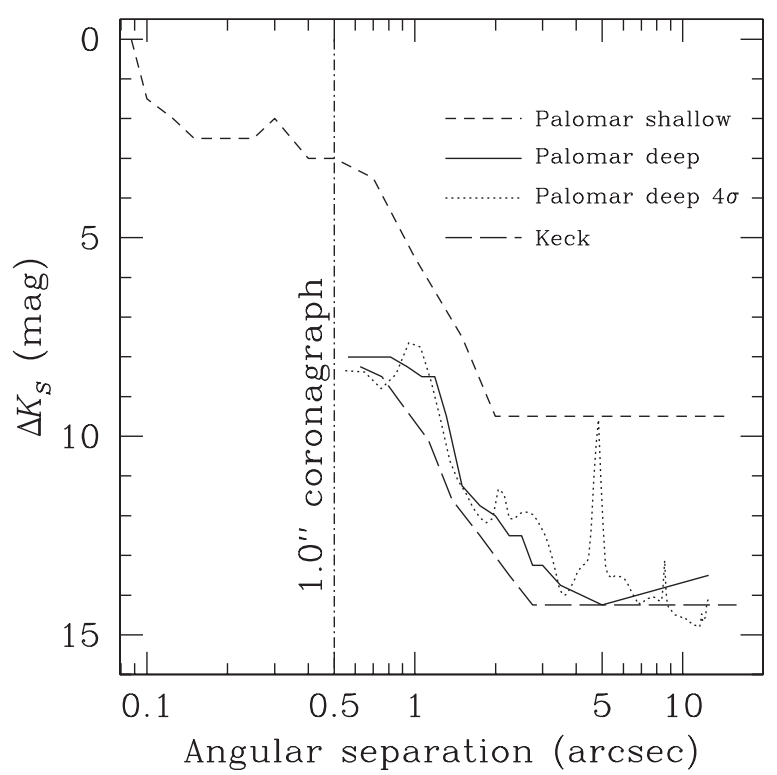

Figure 4. Empirical $K_{S}$-band contrast limits as determined from artificial star experiments in images of the program star HD $172649(V=7.5 \mathrm{mag})$, taken under good $\mathrm{AO}$ performance $(\approx 50 \%$ Strehl ratio). The solid and longdashed curves delineate coronagraphic observations at Palomar (24 min) and Keck (6 min), respectively. The short-dashed line shows the noncoronagraphic component of the Palomar survey. The dotted line represents the $4 \sigma \mathrm{rms}$ deviation of counts in the PSF halo as a function of separation, normalized to an aperture with radius $0{ }^{\prime} .1$ : equal to the FWHM of the $K_{S}$-band PALAO PSF. The vertical dash-dotted line shows the edge of the occulting spot at Palomar and Keck. The slight decrease in contrast in the Palomar coronagraphic limits at $>5^{\prime \prime}$ separations is due to an additive parameter used to model the decreasing exposure depth toward the edge of the PHARO field, because of image misregistration among the different CR angles (Section 3.1.1). The contrast degradation is set to vary between 0 mag and 0.75 mag in the 4 ." 0 12 .' 5 separation range. The bumps and spikes in the rms limits correspond to bright features in the image of HD 172649, such as the corners of the waffle pattern at $1^{\prime \prime} .0$ and projected companions to the star at 2 ". 1,4 .' 8 , and $8^{\prime \prime} .6$.

the thus-obtained $4 \sigma$ aperture-normalized rms noise profile of the halo for our PALAO coronagraphic image of HD 172649 with a dotted line in Figure 4. We found that the $4 \sigma$ line best approximated the visually determined PALAO detection limits. The strongest systematic deviation of the $4 \sigma$ rms profile from the visually determined contrast limits is at angular separations $>7^{\prime \prime}$. This is to be expected, to some extent, because in this region we have adjusted the visual detection limits to account for CR angle image misregistration (Section 3.1.1).

The agreement between the detection limits from visual inspection and from rms statistics is dependent on a number of factors, such as the radius of the normalization aperture, the PSF pixel sampling, the treatment of point-source photon statistics (ignored in our rms analysis), and the appropriate functional treatment of non-Gaussian sources of error (speckles, shape of the PSF core, and halo; also ignored here). As a check on whether the adopted rms detection limit approach was a valid approximation across the range of PSF and image characteristics encountered in our survey, we repeated the artificial point-source experiment on six additional images of targets observed both at Palomar and at Keck. These images were taken under a range of seeing conditions, resulting in PSF Strehl ratios between 10\% and $50 \%$. We found that, on average, the by-eye detection limits varied between three and five times the level of the aperturenormalized rms noise profile over the entire range $\left(0,5-12^{\prime \prime} .5\right)$ of probed angular separations. Thus, the additional experiments confirmed that our choice of the $4 \sigma$ level was an adequate detection threshold.
In closing, we note that because our image noise statistics in the contrast-limited regime are not Gaussian, the adoption of a $4 \sigma$ threshold does not carry the statistical significance of a confidence level at which $99.997 \%$ of random fluctuations are rejected. Marois et al. (2008) have only recently shown that quasi-Gaussian behavior of the PSF subtraction residuals can be obtained with the ADI technique, allowing such formal estimates on the detection limits. Because our data were not taken in the ADI mode, the same formalism cannot be applied here.

\subsection{Illustrative Detection Limits for the Deep Sample}

Table 6 lists the attained point-source magnitude sensitivity for each star in the deep sample at angular separations of $1^{\prime \prime}, 2^{\prime \prime}$, and $5^{\prime \prime}$. Beyond $5^{\prime \prime}$, the detection limits are constant to within 0.5 mag. In the cases where multiple images of the same star were taken at different epochs, we have listed the sensitivity only for the epoch with the deepest image. We thus formed a set of 58 Palomar and 42 Keck images that represented the deepest observations of the 100 stars in the deep sample.

Figure 5(a) depicts the range of attained $K_{S}$-band contrast for the coronagraphic observations in the entire survey (thick solid line), and from the Palomar (dotted line) and Keck (dashed line) portions of it. Figure 5(b) uses the same notation to depict the imaging depth of the survey in terms of apparent $K_{S}$ magnitude (i.e., with the magnitude of the primary added in each case). The median sensitivities of the combined survey range from $\Delta K_{S}=8.4 \mathrm{mag}$ at $1^{\prime \prime}$ to $\Delta K_{S} \approx 12.5 \mathrm{mag}$ over $4^{\prime \prime}-12^{\prime \prime} .5 \mathrm{in}$ contrast and from $K_{S}=15.4 \mathrm{mag}$ at $1^{\prime \prime}$ to $K_{S} \approx 19.7 \mathrm{mag}$ in depth. These detection limits are used in the Appendix to estimate the completeness of the deep survey to substellar and stellar companions.

We obtained the detection limits for the shallow sample in a manner similar to that used for the deep sample: from the $4 \sigma$ dispersion of the radial profile of each star, normalized to an aperture with radius equivalent to the FWHM of the PALAO PSF. The shallow sample detection limits are given in Table 7, where we have in addition listed the sensitivity at 0.5 .

In some cases, close binary companions elevate the dispersion in the radial profile of the primary, resulting in unusually low sensitivities at certain angular separations, for example, for HD 172649 at $5^{\prime \prime}$ in Table 6 and for HD 224873 at $2^{\prime \prime}$ in Table 7 . We have retained these lower sensitivities in Tables 6 and 7 as an indication that part of the images around the sample stars in question were compromised by a nearby bright companion.

\section{CONFIRMATION OF CANDIDATE COMPANIONS}

\subsection{Detected Candidate Companions}

In the course of the 3 year survey we discovered 287 candidate companions brighter than $K_{S}=20.6 \mathrm{mag}$ within 12 .'5 of 130 from the 266 sample stars. Of these candidate companions, 196 were around 61 of the 100 stars in the deep sample. The remaining 91 were in the vicinity of 70 of the 166 shallow-sample targets. All candidate companions around stars in the deep and shallow samples are listed in Tables 8 and 9, respectively. Figure 6 shows all detected candidate companions as a function of magnitude difference $\Delta K_{S}$ and angular separation $\rho$. Thirty-nine stars in the deep sample and 96 in the shallow sample showed no projected companions within $12^{\prime \prime} .5$. 
Table 6

Deep Sample Observations and Sensitivity Limits

\begin{tabular}{|c|c|c|c|c|c|c|}
\hline \multirow[t]{2}{*}{ Star } & \multirow{2}{*}{$\begin{array}{l}\text { Date } \\
\text { (UT) }\end{array}$} & \multirow[t]{2}{*}{ Observatory } & \multirow[t]{2}{*}{ Optic ${ }^{b}$} & \multicolumn{3}{|c|}{$\begin{array}{c}\text { Limiting } K_{S} \text {-Band } \\
\text { Magnitude at }{ }^{\mathrm{a}}\end{array}$} \\
\hline & & & & $1^{\prime \prime}$ & $2^{\prime \prime}$ & $5^{\prime \prime}$ \\
\hline \multirow[t]{3}{*}{ HD 377} & 2002 Aug 28 & Palomar & Corona & $\ldots$ & $\ldots$ & $\ldots$ \\
\hline & 2003 Nov 09 & Keck & Corona & 16.7 & 18.3 & 20.0 \\
\hline & 2004 Oct 07 & Keck & Corona & $\ldots$ & $\ldots$ & $\ldots$ \\
\hline \multirow[t]{2}{*}{ HD 691} & 2002 Aug 31 & Palomar & Corona & $\ldots$ & $\ldots$ & $\ldots$ \\
\hline & 2002 Nov 18 & Palomar & Corona & 15.1 & 18.3 & 20.3 \\
\hline \multirow[t]{3}{*}{ HD 984} & 2002 Aug 29 & Palomar & Corona & 13.1 & 16.9 & 18.5 \\
\hline & 2003 Sep 20 & Palomar & Corona & $\ldots$ & $\ldots$ & $\ldots$ \\
\hline & 2004 Oct 04 & Palomar & Corona & $\ldots$ & $\ldots$ & $\ldots$ \\
\hline HD 1405 & 2004 Jun 06 & Keck & Corona & 16.3 & 18.3 & 19.2 \\
\hline \multirow[t]{3}{*}{ QT And } & 2002 Aug 29 & Palomar & Corona & $\ldots$ & $\ldots$ & $\ldots$ \\
\hline & 2003 Nov 10 & Keck & Corona & 17.6 & 19.1 & 19.9 \\
\hline & 2004 Oct 07 & Keck & Corona & $\ldots$ & $\ldots$ & $\ldots$ \\
\hline \multirow[t]{2}{*}{ HD 7661} & 2002 Aug 30 & Palomar & Corona & $\ldots$ & $\ldots$ & $\ldots$ \\
\hline & 2002 Nov 09 & Keck & Corona & 16.5 & 18.0 & 20.0 \\
\hline \multirow[t]{2}{*}{ HIP 6276} & 2002 Aug 30 & Palomar & Corona & 14.5 & 18.9 & 20.7 \\
\hline & 2004 Oct 08 & Keck & Corona & $\ldots$ & $\ldots$ & $\ldots$ \\
\hline HD 8907 & 2002 Aug 27 & Palomar & Corona & 13.5 & 17.3 & 19.3 \\
\hline HD 12039 & 2002 Aug 28 & Palomar & Corona & 14.0 & 18.5 & 20.0 \\
\hline HD 15526 & 2003 Sep 20 & Palomar & Corona & 15.5 & 19.4 & 20.3 \\
\hline \multirow[t]{3}{*}{ 1RXS J025216.9+361658 } & 2002 Nov 18 & Palomar & Corona & $\ldots$ & $\ldots$ & $\cdots$ \\
\hline & 2003 Nov 10 & Keck & Corona & 17.8 & 19.3 & 20.3 \\
\hline & 2004 Feb 07 & Palomar & Corona & $\ldots$ & $\ldots$ & $\cdots$ \\
\hline HD 17925 & 2003 Sep 21 & Palomar & Corona & 12.5 & 15.3 & 18.6 \\
\hline 1RXS J025751.8+115759 & 2002 Nov 16 & Palomar & Corona & 16.4 & 19.8 & 20.5 \\
\hline RX J0258.4+2947 & 2002 Feb 28 & Palomar & Corona & 17.7 & 19.5 & 19.6 \\
\hline 1RXS J030759.1+302032 & 2002 Nov 18 & Palomar & Corona & 15.1 & 18.2 & 19.8 \\
\hline \multirow[t]{2}{*}{ HD 19668} & 2002 Aug 27 & Palomar & Corona & $\ldots$ & $\ldots$ & $\ldots$ \\
\hline & 2003 Nov 09 & Keck & Corona & 17.0 & 18.5 & 20.0 \\
\hline 1E $0307.4+1424$ & 2003 Jan 12 & Palomar & Corona & 15.6 & 18.9 & 20.5 \\
\hline \multirow[t]{3}{*}{ V525 Per } & 2003 Sep 20 & Palomar & $\ldots$ & $\cdots$ & $\ldots$ & $\cdots$ \\
\hline & 2004 Oct 03 & Palomar & $\ldots$ & $\ldots$ & $\ldots$ & $\ldots$ \\
\hline & 2004 Oct 08 & Keck & Corona & 18.7 & 20.3 & 20.4 \\
\hline 1RXS J031907.4+393418 & 2002 Aug 29 & Palomar & Corona & $\ldots$ & $\ldots$ & $\ldots$ \\
\hline & 2003 Nov 10 & Keck & Corona & 19.3 & 20.5 & 20.7 \\
\hline & 2004 Oct 08 & Keck & Corona & $\cdots$ & $\cdots$ & $\cdots$ \\
\hline HE 622 & 2003 Sep 20 & Palomar & $\ldots$ & $\ldots$ & $\ldots$ & $\cdots$ \\
\hline & 2004 Oct 08 & Keck & Corona & 19.2 & 20.8 & 20.9 \\
\hline 1E 0324.1-2012 & 2003 Jan 12 & Palomar & Corona & $\ldots$ & $\ldots$ & $\ldots$ \\
\hline & 2004 Feb 07 & Palomar & Corona & $\ldots$ & $\ldots$ & $\ldots$ \\
\hline & 2004 Oct 08 & Keck & Corona & 18.1 & 19.5 & 19.8 \\
\hline RX J0329.1+0118 & 2003 Sep 21 & Palomar & Corona & 16.6 & 19.7 & 20.1 \\
\hline HE 1101 & 2003 Sep 20 & Palomar & $\cdots$ & $\ldots$ & $\ldots$ & $\cdots$ \\
\hline & 2004 Oct 03 & Palomar & $\ldots$ & $\ldots$ & $\ldots$ & $\ldots$ \\
\hline & 2004 Oct 07 & Keck & Corona & 19.2 & 20.9 & 21.0 \\
\hline & 2006 Dec 12 & Keck & Corona & $\ldots$ & $\ldots$ & $\ldots$ \\
\hline HD 22179 & 2002 Nov 16 & Palomar & Corona & $\ldots$ & $\ldots$ & $\ldots$ \\
\hline & 2003 Nov 09 & Keck & Corona & 18.1 & 19.4 & 20.2 \\
\hline & 2004 Feb 07 & Palomar & Corona & $\ldots$ & $\ldots$ & $\ldots$ \\
\hline & 2004 Oct 08 & Keck & Corona & $\ldots$ & $\ldots$ & $\ldots$ \\
\hline HD 23208 & 2004 Oct 05 & Palomar & Corona & 14.9 & 19.2 & 20.1 \\
\hline H II 120 & 2003 Sep 20 & Palomar & $\ldots$ & $\ldots$ & $\cdots$ & $\cdots$ \\
\hline & 2004 Oct 04 & Palomar & $\cdots$ & $\ldots$ & $\ldots$ & $\ldots$ \\
\hline & 2004 Oct 08 & Keck & Corona & 18.6 & 20.0 & 20.2 \\
\hline & 2006 Jan 18 & Palomar & $\ldots$ & $\ldots$ & $\ldots$ & $\ldots$ \\
\hline H II 2147 & 2003 Jan 13 & Palomar & Corona & 15.2 & 19.3 & 20.6 \\
\hline 1RXS J035028.0+163121 & 2002 Nov 17 & Palomar & Corona & 16.7 & 19.9 & 20.3 \\
\hline RX J0354.4+0535 & 2003 Jan 13 & Palomar & Corona & $\cdots$ & $\cdots$ & $\cdots$ \\
\hline & 2004 Feb 07 & Palomar & $\ldots$ & $\ldots$ & $\ldots$ & $\ldots$ \\
\hline & 2004 Oct 07 & Keck & Corona & 18.3 & 19.9 & 20.3 \\
\hline HD 283167 & 2002 Nov 18 & Palomar & Corona & 17.0 & 19.6 & 19.8 \\
\hline RX J0357.3+1258 & 2003 Jan 11 & Palomar & Corona & $\ldots$ & $\ldots$ & $\ldots$ \\
\hline & 2003 Nov 09 & Keck & Corona & 18.9 & 20.3 & 20.5 \\
\hline
\end{tabular}


Table 6

(Continued)

\begin{tabular}{|c|c|c|c|c|c|c|}
\hline \multirow[t]{2}{*}{ Star } & \multirow{2}{*}{$\begin{array}{l}\text { Date } \\
\text { (UT) }\end{array}$} & \multirow[t]{2}{*}{ Observatory } & \multirow[t]{2}{*}{ Optic $^{b}$} & \multicolumn{3}{|c|}{$\begin{array}{l}\text { Limiting } K_{S}-\text { Band } \\
\text { Magnitude at }{ }^{\mathrm{a}}\end{array}$} \\
\hline & & & & $1^{\prime \prime}$ & $2^{\prime \prime}$ & $5^{\prime \prime}$ \\
\hline & 2004 Feb 07 & Palomar & Corona & $\ldots$ & $\ldots$ & \\
\hline HD 285751 & 2002 Jan 31 & Palomar & Corona & 15.7 & 18.1 & 19.2 \\
\hline \multirow[t]{2}{*}{ RX J0442.5+0906 } & 2003 Jan 11 & Palomar & Corona & 16.4 & 19.5 & 20.0 \\
\hline & $2004 \mathrm{Feb} 07$ & Palomar & $\ldots$ & $\ldots$ & $\ldots$ & $\ldots$ \\
\hline \multirow[t]{4}{*}{ HD 286179} & 2002 Jan 31 & Palomar & Corona & $\ldots$ & $\ldots$ & $\ldots$ \\
\hline & 2004 Feb 07 & Palomar & & $\ldots$ & $\ldots$ & $\ldots$ \\
\hline & 2004 Oct 07 & Keck & Corona & 17.8 & 19.6 & 20.3 \\
\hline & 2006 Dec 12 & Keck & Corona & $\ldots$ & $\ldots$ & $\ldots$ \\
\hline \multirow[t]{4}{*}{ HD 31950} & 2002 Nov 16 & Palomar & Corona & $\ldots$ & $\ldots$ & $\ldots$ \\
\hline & 2003 Nov 09 & Keck & Corona & 18.1 & 19.4 & 19.8 \\
\hline & 2004 Feb 07 & Palomar & Corona & $\ldots$ & $\ldots$ & $\ldots$ \\
\hline & 2004 Oct 08 & Keck & Corona & $\ldots$ & $\ldots$ & $\ldots$ \\
\hline HD 35850 & 2002 Feb 01 & Palomar & Corona & 11.7 & 14.3 & 16.9 \\
\hline \multirow[t]{3}{*}{ 1RXS J053650.0+133756 } & $2002 \mathrm{Feb} 28$ & Palomar & Corona & $\ldots$ & $\ldots$ & $\ldots$ \\
\hline & 2003 Jan 14 & Palomar & Corona & & $\ldots$ & \\
\hline & 2003 Nov 10 & Keck & Corona & 18.4 & 18.0 & 20.6 \\
\hline \multirow[t]{4}{*}{ HD 245567} & 2002 Nov 16 & Palomar & Corona & $\ldots$ & $\ldots$ & $\ldots$ \\
\hline & 2003 Nov 09 & Keck & Corona & $\ldots$ & $\ldots$ & $\ldots$ \\
\hline & $2004 \mathrm{Feb} 07$ & Palomar & Corona & $\ldots$ & $\ldots$ & $\ldots$ \\
\hline & 2004 Oct 08 & Keck & Corona & 16.7 & 18.6 & 19.6 \\
\hline \multirow[t]{3}{*}{ SAO 150676} & 2002 Nov 17 & Palomar & Corona & $\ldots$ & $\ldots$ & $\ldots$ \\
\hline & 2004 Feb 07 & Palomar & Corona & $\ldots$ & $\ldots$ & $\ldots$ \\
\hline & 2004 Oct 08 & Keck & Corona & 16.8 & 18.5 & 19.0 \\
\hline HD 38949 & 2002 Nov 18 & Palomar & Corona & 13.7 & 17.4 & 19.5 \\
\hline HD 43989 & 2004 Feb 07 & Palomar & Corona & 13.6 & 16.3 & 19.0 \\
\hline \multirow[t]{3}{*}{ HD 49197} & 2002 Feb 28 & Palomar & Corona & $\ldots$ & $\ldots$ & $\ldots$ \\
\hline & 2003 Nov 09 & Keck & Corona & 16.1 & 17.8 & 18.9 \\
\hline & $2004 \mathrm{Feb} 07$ & Palomar & Corona & $\ldots$ & $\ldots$ & $\ldots$ \\
\hline \multirow[t]{3}{*}{ RE J0723+20 } & $2002 \mathrm{Feb} 28$ & Palomar & Corona & 15.3 & 19.2 & 19.9 \\
\hline & 2003 Jan 13 & Palomar & $\ldots$ & $\ldots$ & $\ldots$ & $\ldots$ \\
\hline & $2004 \mathrm{Feb} 05$ & Palomar & $\ldots$ & $\ldots$ & $\ldots$ & $\ldots$ \\
\hline \multirow[t]{3}{*}{ HD 60737} & 2002 Jan 31 & Palomar & Corona & $\ldots$ & $\ldots$ & $\ldots$ \\
\hline & 2003 Jan 11 & Palomar & Corona & $\ldots$ & $\ldots$ & $\ldots$ \\
\hline & 2003 Nov 10 & Keck & Corona & 16.7 & 18.1 & 19.5 \\
\hline HD 70573 & 2002 Feb 01 & Palomar & Corona & 14.2 & 17.8 & 19.6 \\
\hline HD 70516 & 2002 Jan 31 & Palomar & Corona & $\ldots$ & $\ldots$ & $\ldots$ \\
\hline & 2002 Nov 16 & Palomar & Corona & 13.9 & 17.7 & 19.8 \\
\hline HD 72905 & $2002 \mathrm{Feb} 28$ & Palomar & Corona & 12.8 & 16.7 & 18.5 \\
\hline HD 75393 & 2002 Nov 17 & Palomar & Corona & 13.8 & 17.7 & 19.8 \\
\hline HD 82558 & 2003 Dec 09 & Palomar & Corona & 12.9 & 16.6 & 19.5 \\
\hline HD 82443 & 2004 Feb 07 & Palomar & Corona & 12.6 & 16.2 & 19.3 \\
\hline SAO 178272 & 2003 Jan 13 & Palomar & Corona & 14.2 & 17.8 & 19.7 \\
\hline & $2004 \mathrm{Feb} 07$ & Palomar & Corona & $\ldots$ & $\ldots$ & $\ldots$ \\
\hline HD 90905 & 2002 Feb 01 & Palomar & Corona & $\ldots$ & $\ldots$ & $\ldots$ \\
\hline & 2003 Jan 12 & Palomar & Corona & $\ldots$ & $\ldots$ & $\ldots$ \\
\hline & 2004 Feb 05 & Palomar & Corona & $\ldots$ & $\ldots$ & $\ldots$ \\
\hline & 2004 Jun 05 & Keck & Corona & 15.4 & 17.4 & 18.8 \\
\hline HD 91782 & 2002 Mar 02 & Palomar & Corona & $\ldots$ & $\ldots$ & $\ldots$ \\
\hline & 2003 Jan 11 & Palomar & Corona & $\ldots$ & $\ldots$ & $\ldots$ \\
\hline & 2003 May 10 & Palomar & Corona & $\ldots$ & $\ldots$ & $\ldots$ \\
\hline & $2004 \mathrm{Feb} 05$ & Palomar & Corona & $\ldots$ & $\ldots$ & $\ldots$ \\
\hline & 2004 Jun 05 & Keck & Corona & $\ldots$ & $\ldots$ & $\ldots$ \\
\hline & 2004 Jun 06 & Keck & Corona & 13.0 & 18.5 & 18.8 \\
\hline HD 92855 & 2002 Feb 01 & Palomar & Corona & $\ldots$ & $\ldots$ & $\ldots$ \\
\hline & 2003 Jan 13 & Palomar & Corona & $\ldots$ & $\ldots$ & $\ldots$ \\
\hline & 2004 Feb 06 & Palomar & Corona & $\ldots$ & $\ldots$ & $\ldots$ \\
\hline & 2004 Jun 06 & Keck & Corona & 15.7 & 17.5 & 18.2 \\
\hline & 2004 Jun 26 & Palomar & Corona & $\ldots$ & $\ldots$ & $\ldots$ \\
\hline HD 93528 & $2004 \mathrm{Feb} 07$ & Palomar & Corona & 13.4 & 17.1 & 19.7 \\
\hline HD 95188 & 2002 Mar 02 & Palomar & Corona & 13.5 & 16.4 & 18.8 \\
\hline HD 101472 & 2002 Mar 02 & Palomar & Corona & $\ldots$ & $\ldots$ & $\ldots$ \\
\hline & 2003 Jan 14 & Palomar & Corona & 12.3 & 16.5 & 19.2 \\
\hline
\end{tabular}


Table 6

(Continued)

\begin{tabular}{|c|c|c|c|c|c|c|}
\hline \multirow[t]{2}{*}{ Star } & \multirow{2}{*}{$\begin{array}{l}\text { Date } \\
\text { (UT) }\end{array}$} & \multirow[t]{2}{*}{ Observatory } & \multirow[t]{2}{*}{ Optic $^{b}$} & \multicolumn{3}{|c|}{$\begin{array}{l}\text { Limiting } K_{S} \text {-Band } \\
\text { Magnitude at }{ }^{\mathrm{a}}\end{array}$} \\
\hline & & & & $1^{\prime \prime}$ & $2^{\prime \prime}$ & $5^{\prime \prime}$ \\
\hline \multirow[t]{3}{*}{ GQ Leo } & 2003 Jan 12 & Palomar & Corona & 14.9 & 18.9 & 20.8 \\
\hline & 2003 Jul 16 & Palomar & $\ldots$ & $\ldots$ & $\ldots$ & $\ldots$ \\
\hline & 2004 Feb 05 & Palomar & $\ldots$ & $\ldots$ & $\ldots$ & $\ldots$ \\
\hline HD 104576 & 2002 Jun 22 & Palomar & Corona & 14.0 & 18.6 & 20.0 \\
\hline \multirow[t]{3}{*}{ HD 104860} & 2002 Jun 23 & Palomar & Corona & $\ldots$ & $\ldots$ & $\ldots$ \\
\hline & 2003 May 11 & Palomar & Corona & $\ldots$ & $\ldots$ & $\ldots$ \\
\hline & 2004 Jun 05 & Keck & Corona & 16.7 & 18.5 & 19.8 \\
\hline \multirow[t]{2}{*}{ HD 107146} & 2002 Jan 31 & Palomar & Corona & $\ldots$ & $\ldots$ & $\ldots$ \\
\hline & 2003 Jan 14 & Palomar & Corona & 12.7 & 16.6 & 19.3 \\
\hline \multirow[t]{2}{*}{ SAO 15880} & 2004 Feb 06 & Palomar & Corona & $\ldots$ & $\ldots$ & $\ldots$ \\
\hline & 2004 Jun 06 & Keck & Corona & 17.4 & 19.0 & 19.6 \\
\hline SAO 2085 & 2004 Feb 07 & Palomar & Corona & 14.9 & 18.6 & 20.7 \\
\hline HD 111456 & $2004 \mathrm{Feb} 06$ & Palomar & Corona & 11.7 & 15.5 & 18.4 \\
\hline \multirow[t]{2}{*}{ HD 132173} & 2002 Feb 28 & Palomar & Corona & $\ldots$ & $\ldots$ & $\ldots$ \\
\hline & 2004 Feb 07 & Palomar & Corona & 13.9 & 17.4 & 20.2 \\
\hline \multirow[t]{3}{*}{ HD 139813} & 2002 Feb 01 & Palomar & Corona & $\ldots$ & $\ldots$ & $\ldots$ \\
\hline & 2003 May 17 & Keck & Corona & $\ldots$ & $\ldots$ & $\ldots$ \\
\hline & 2003 May 18 & Keck & Corona & 15.9 & 16.8 & 18.7 \\
\hline \multirow[t]{2}{*}{ HD 139498} & $2003 \mathrm{Jul} 15$ & Palomar & $\ldots$ & $\ldots$ & $\ldots$ & $\ldots$ \\
\hline & 2004 Jun 26 & Palomar & Corona & 15.9 & 19.7 & 20.5 \\
\hline \multirow[t]{3}{*}{ HD 142361} & 2002 Jun 21 & Palomar & Corona & 13.6 & 17.2 & 18.6 \\
\hline & $2003 \mathrm{Jul} 16$ & Palomar & ND1 & $\ldots$ & $\ldots$ & $\ldots$ \\
\hline & 2004 Jun 27 & Palomar & ND1 & $\ldots$ & $\ldots$ & $\ldots$ \\
\hline HD 143006 & 2002 Jun 23 & Palomar & Corona & $\ldots$ & $\ldots$ & $\ldots$ \\
\hline & 2003 May 18 & Keck & Corona & $\ldots$ & $\ldots$ & $\ldots$ \\
\hline & 2004 Jun 05 & Keck & Corona & 17.2 & 19.0 & 20.4 \\
\hline PZ99 J155847.8-175800 & 2004 Jun 06 & Keck & Corona & 17.2 & 19.4 & 20.3 \\
\hline ScoPMS 21 & 2002 Jun 22 & Palomar & Corona & $\ldots$ & $\ldots$ & $\ldots$ \\
\hline & 2004 Jun 05 & Keck & Corona & 18.4 & 20.3 & 20.6 \\
\hline PZ99 J160158.2-200811 & 2002 Aug 29 & Palomar & Corona & 15.4 & 19.4 & 20.5 \\
\hline PZ99 J160302.7-180605 & 2003 May 18 & Keck & Corona & $\ldots$ & $\ldots$ & $\ldots$ \\
\hline & 2004 Jun 06 & Keck & Corona & 17.9 & 20.0 & 20.4 \\
\hline ScoPMS 27 & 2004 Jun 28 & Palomar & Corona & 16.6 & 20.3 & 20.5 \\
\hline ScoPMS 52 & 2002 Aug 31 & Palomar & Corona & 14.8 & 18.2 & 20.2 \\
\hline & 2004 Jun 26 & Palomar & & $\ldots$ & $\ldots$ & $\ldots$ \\
\hline PZ99 J161318.6-221248 & 2002 Jun 21 & Palomar & Corona & $\ldots$ & $\ldots$ & $\ldots$ \\
\hline & 2003 May 18 & Keck & Corona & $\ldots$ & $\ldots$ & $\ldots$ \\
\hline & 2004 Jun 05 & Keck & Corona & 17.4 & 19.2 & 20.1 \\
\hline PZ99 J161402.1-230101 & 2003 Jul 15 & Palomar & $\ldots$ & $\ldots$ & $\ldots$ & $\ldots$ \\
\hline & 2004 Jun 05 & Keck & Corona & 18.5 & 20.3 & 20.7 \\
\hline PZ99 J161411.0-230536 & 2002 Jun 21 & Palomar & Corona & $\ldots$ & $\ldots$ & $\ldots$ \\
\hline & 2004 Jun 05 & Keck & Corona & 17.6 & 19.4 & 20.2 \\
\hline PZ99 J161459.2-275023 & 2003 Jul 15 & Palomar & $\cdots$ & $\ldots$ & $\ldots$ & $\ldots$ \\
\hline & 2004 Jun 05 & Keck & Corona & 18.4 & 20.2 & 20.2 \\
\hline PZ99 J161618.0-233947 & 2004 Jun 27 & Palomar & Corona & 16.3 & 20.3 & 20.6 \\
\hline HD 146516 & 2003 May 10 & Palomar & $\ldots$ & $\ldots$ & $\ldots$ & $\ldots$ \\
\hline & 2004 Jun 05 & Keck & Corona & 17.6 & 19.5 & 20.1 \\
\hline ScoPMS 214 & 2002 Aug 30 & Palomar & Corona & $\ldots$ & $\ldots$ & $\ldots$ \\
\hline & 2004 Jun 05 & Keck & Corona & 17.6 & 19.4 & 20.1 \\
\hline & 2004 Jun 27 & Palomar & Corona & $\ldots$ & $\ldots$ & $\ldots$ \\
\hline & 2007 Jun 23 & Keck & Corona & $\ldots$ & $\ldots$ & $\ldots$ \\
\hline HD 151798 & 2002 Jun 21 & Palomar & Corona & $\ldots$ & $\ldots$ & $\ldots$ \\
\hline & 2003 May 16 & Keck & Corona & 17.6 & 18.7 & 20.0 \\
\hline & 2004 Jun 26 & Palomar & Corona & $\ldots$ & $\ldots$ & $\ldots$ \\
\hline HD 165590 & 2004 Jun 28 & Palomar & Corona & 14.2 & 17.7 & 20.4 \\
\hline & 2007 Jun 23 & Keck & Corona & $\ldots$ & $\ldots$ & $\ldots$ \\
\hline HD 166181 & 2004 Jun 06 & Keck & Corona & 15.5 & 17.4 & 18.8 \\
\hline HD 170778 & 2004 Jun 27 & Palomar & Corona & 14.2 & 18.5 & 20.5 \\
\hline HD 171488 & 2004 Jun 06 & Keck & Corona & 15.3 & 17.2 & 18.9 \\
\hline & 2004 Oct 08 & Keck & Corona & $\ldots$ & $\ldots$ & $\ldots$ \\
\hline HD 172649 & 2002 Jun 21 & Palomar & $\ldots$ & $\ldots$ & $\ldots$ & $\ldots$ \\
\hline & 2002 Aug 31 & Palomar & Corona & 14.0 & 17.6 & 18.5 \\
\hline & 2003 May 13 & Palomar & $\ldots$ & $\ldots$ & $\ldots$ & $\ldots$ \\
\hline
\end{tabular}


Table 6

(Continued)

\begin{tabular}{|c|c|c|c|c|c|c|}
\hline \multirow[t]{2}{*}{ Star } & \multirow{2}{*}{$\begin{array}{l}\text { Date } \\
\text { (UT) }\end{array}$} & \multirow[t]{2}{*}{ Observatory } & \multirow[t]{2}{*}{ Optic $^{b}$} & \multicolumn{3}{|c|}{$\begin{array}{c}\text { Limiting } K_{S} \text {-Band Magnitude } \\
\text { at }^{\mathrm{a}}\end{array}$} \\
\hline & & & & $1^{\prime \prime}$ & $2^{\prime \prime}$ & $5^{\prime \prime}$ \\
\hline & 2003 May 18 & Keck & Corona & $\ldots$ & $\ldots$ & $\ldots$ \\
\hline & 2004 Jun 05 & Keck & Corona & $\ldots$ & $\ldots$ & $\ldots$ \\
\hline \multirow[t]{2}{*}{ HD 187748} & 2004 Jun 27 & Palomar & Corona & 13.4 & 17.8 & 20.4 \\
\hline & 2007 Jun 23 & Keck & Corona & $\ldots$ & $\ldots$ & $\ldots$ \\
\hline \multirow[t]{3}{*}{ HD 191089} & 2003 Sep 20 & Palomar & Corona & $\ldots$ & $\ldots$ & $\ldots$ \\
\hline & 2004 Jun 26 & Palomar & Corona & 14.1 & 18.3 & 20.4 \\
\hline & 2007 Jun 23 & Keck & Corona & $\ldots$ & $\ldots$ & $\ldots$ \\
\hline HD 199019 & 2002 Aug 29 & Palomar & Corona & 14.4 & 18.1 & 20.3 \\
\hline \multirow[t]{4}{*}{ HD 200746} & 2003 Sep 21 & Palomar & Corona & $\ldots$ & $\ldots$ & $\ldots$ \\
\hline & 2004 Jun 26 & Palomar & Corona & 14.4 & 18.1 & 19.9 \\
\hline & 2006 Dec 12 & Keck & Corona & $\ldots$ & $\ldots$ & $\ldots$ \\
\hline & 2007 Jun 23 & Keck & Corona & $\ldots$ & $\ldots$ & $\ldots$ \\
\hline \multirow[t]{3}{*}{ HD 203030} & 2002 Aug 28 & Palomar & Corona & 14.8 & 18.7 & 20.4 \\
\hline & 2003 Jul 16 & Palomar & Corona & $\ldots$ & $\ldots$ & $\ldots$ \\
\hline & 2004 Jun 26 & Palomar & Corona & $\ldots$ & $\ldots$ & $\ldots$ \\
\hline \multirow[t]{3}{*}{ HD 209393} & 2002 Nov 17 & Palomar & Corona & 13.9 & 17.8 & 20.0 \\
\hline & 2003 Sep 20 & Palomar & Corona & $\ldots$ & $\ldots$ & $\ldots$ \\
\hline & 2004 Oct 07 & Keck & Corona & $\ldots$ & $\ldots$ & $\ldots$ \\
\hline HD 209779 & 2002 Nov 16 & Palomar & Corona & 13.2 & 17.2 & 19.4 \\
\hline \multirow[t]{3}{*}{ V383 Lac } & 2002 Aug 27 & Palomar & Corona & $\ldots$ & $\ldots$ & $\ldots$ \\
\hline & 2003 Jul 16 & Palomar & Corona & $\ldots$ & $\ldots$ & $\ldots$ \\
\hline & 2003 Nov 09 & Keck & Corona & 17.0 & 18.4 & 19.8 \\
\hline HD 217343 & 2003 Sep 21 & Palomar & Corona & 13.2 & 17.0 & 19.7 \\
\hline \multirow[t]{2}{*}{ HD 218738} & 2003 Dec 10 & Palomar & Corona & $\ldots$ & $\ldots$ & $\ldots$ \\
\hline & 2004 Oct 04 & Palomar & Corona & 16.3 & 18.8 & 19.8 \\
\hline \multirow[t]{2}{*}{ HD 218739} & 2003 Dec 10 & Palomar & Corona & $\ldots$ & $\ldots$ & $\ldots$ \\
\hline & 2004 Oct 04 & Palomar & Corona & 16.3 & 18.8 & 19.8 \\
\hline \multirow[t]{3}{*}{ HD 219498} & 2002 Aug 30 & Palomar & Corona & 15.7 & 19.7 & 21.1 \\
\hline & 2003 Sep 20 & Palomar & Corona & $\ldots$ & $\ldots$ & $\ldots$ \\
\hline & 2004 Oct 05 & Palomar & Corona & $\ldots$ & $\ldots$ & $\ldots$ \\
\hline
\end{tabular}

Notes.

a The limiting magnitude corresponds to the apparent $K_{S}$-band magnitude of the faintest detectable point source at the given angular separation, and is quoted only for the epoch of the deepest observation.

b "Corona" entries in the column "Optic" indicate that a coronagraph has been used.

(This table is also available in a machine-readable form in the online journal.)
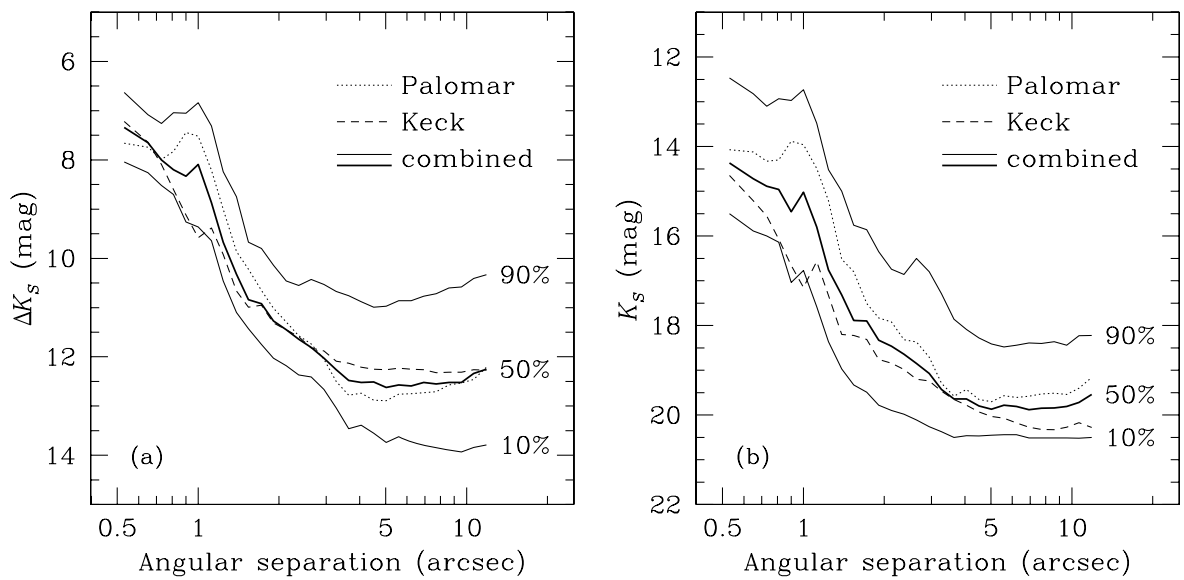

Figure 5. Contrast (a) and depth (b) of the deep survey at $K_{S}$. The solid lines represent the $10 \%, 50 \%$ (thick), and $90 \%$ completeness of the combined Palomar + Keck AO survey. The median (50\%) sensitivities of the Palomar (dotted line) and Keck (dashed line) surveys are also shown. The gradual decrease in imaging contrast and depth at Palomar between 4" and 12'.5 is partially due to misregistration of images taken at different CR angles (Section 3.2), and partially to the sometimes smaller depth of observations at $11^{\prime \prime}-12^{\prime \prime} .5$ separations because of a $0.5-1.5$ offset of the coronagraphic spot from the center of the PHARO array.

\subsection{Deciding Physical Association}

The physical association of each candidate companion was decided based on one of the following criteria: (1) common proper motion with the candidate primary, (2) a combination of the position on a $J-K_{S}$ versus $M_{K_{S}}$ color-absolute-magnitude diagram (CAMD; assuming equidistance with the primary) and background star density arguments, or (3) extent of the radial profile of the candidate companion beyond that of a point- 
Table 7

Shallow Sample Observations and Sensitivity Limits

\begin{tabular}{|c|c|c|c|c|c|c|c|}
\hline \multirow[t]{2}{*}{ Star } & \multirow{2}{*}{$\begin{array}{l}\text { Date } \\
\text { (UT) }\end{array}$} & \multirow[t]{2}{*}{ Observatory } & \multirow[t]{2}{*}{ Optic $^{\mathrm{b}}$} & \multicolumn{4}{|c|}{ Limiting $K_{S}$-Band Magnitude at ${ }^{\mathrm{a}}$} \\
\hline & & & & $0 ! .5$ & $1^{\prime \prime}$ & $2^{\prime \prime}$ & $5^{\prime \prime}$ \\
\hline \multirow[t]{2}{*}{ HD 224873} & 2002 Aug 31 & Palomar & ND1 & $\ldots$ & $\ldots$ & $\ldots$ & $\ldots$ \\
\hline & 2003 Sep 21 & Palomar & ND1 & 11.8 & 13.5 & 10.0 & 14.7 \\
\hline HD 6963 & 2004 Jun 04 & Palomar & ND1 & 10.3 & 11.9 & 13.8 & 14.1 \\
\hline HD 8467 & 2004 Jun 04 & Palomar & ND1 & 10.8 & 12.4 & 13.6 & 13.9 \\
\hline HD 8941 & 2004 Jun 04 & Palomar & ND1 & 9.8 & 11.3 & 13.5 & 14.0 \\
\hline \multirow[t]{3}{*}{ HD 9472} & 2002 Nov 18 & Palomar & ND1 & $\ldots$ & $\ldots$ & $\ldots$ & $\ldots$ \\
\hline & 2003 Sep 20 & Palomar & ND1 & $\ldots$ & $\ldots$ & $\ldots$ & $\ldots$ \\
\hline & 2004 Oct 05 & Palomar & ND1 & 10.8 & 12.6 & 15.1 & 15.8 \\
\hline \multirow[t]{2}{*}{ RE J0137+18A } & 2002 Jan 31 & Palomar & & 11.7 & 13.2 & 12.0 & 14.9 \\
\hline & 2003 Sep 20 & Palomar & ND1 & $\ldots$ & $\ldots$ & $\ldots$ & $\ldots$ \\
\hline HD 11850 & 2003 Sep 21 & Palomar & ND1 & 10.7 & 12.4 & 14.8 & 15.3 \\
\hline HD 13382 & 2004 Jun 04 & Palomar & ND1 & 10.4 & 12.2 & 14.7 & 15.0 \\
\hline HD 13507 & 2002 Aug 28 & Palomar & Corona & 11.7 & 13.8 & 15.6 & 18.1 \\
\hline \multirow[t]{3}{*}{ HD 13531} & 2002 Aug 28 & Palomar & ND1 & $\ldots$ & $\ldots$ & $\ldots$ & $\ldots$ \\
\hline & 2003 Sep 21 & Palomar & ND1 & $\ldots$ & $\ldots$ & $\ldots$ & $\ldots$ \\
\hline & 2004 Oct 05 & Palomar & ND1 & 10.4 & 12.1 & 14.7 & 15.6 \\
\hline HD 13974 & 2004 Jun 04 & Palomar & ND1 & 7.6 & 9.2 & 11.8 & 13.5 \\
\hline \multirow[t]{2}{*}{ 1RXS J025223.5+372914 } & 2003 Sep 21 & Palomar & $\ldots$ & $\ldots$ & $\ldots$ & $\ldots$ & $\ldots$ \\
\hline & 2004 Oct 05 & Palomar & $\ldots$ & 14.1 & 12.8 & 17.6 & 18.2 \\
\hline \multirow[t]{2}{*}{ 2RE J0255+474 } & $2002 \mathrm{Feb} 28$ & Palomar & $\ldots$ & $\ldots$ & $\ldots$ & $\ldots$ & $\ldots$ \\
\hline & 2004 Oct 05 & Palomar & & 12.5 & 14.9 & 15.8 & 16.7 \\
\hline HD 18940 & 2002 Aug 29 & Palomar & ND1 & 9.9 & 11.9 & 14.8 & 15.1 \\
\hline HD 19019 & 2004 Jun 04 & Palomar & ND1 & 9.9 & 12.0 & 14.6 & 14.9 \\
\hline HD 19632 & 2002 Aug 30 & Palomar & $\ldots$ & 9.8 & 12.0 & 14.2 & 14.5 \\
\hline vB 1 & 2002 Aug 29 & Palomar & ND1 & 10.3 & 12.2 & 14.6 & 14.8 \\
\hline \multirow[t]{2}{*}{ HE 350} & 2003 Sep 20 & Palomar & $\ldots$ & $\ldots$ & $\ldots$ & $\ldots$ & $\ldots$ \\
\hline & 2004 Oct 04 & Palomar & $\ldots$ & 13.7 & 15.9 & 18.1 & 18.3 \\
\hline HE 373 & 2003 Sep 20 & Palomar & $\ldots$ & $\ldots$ & $\ldots$ & $\ldots$ & $\ldots$ \\
\hline & 2003 Nov 10 & Keck & $\ldots$ & $\ldots$ & $\ldots$ & $\ldots$ & $\ldots$ \\
\hline & 2004 Oct 05 & Palomar & $\ldots$ & 13.8 & 16.1 & 18.0 & 18.2 \\
\hline HE 389 & 2003 Sep 20 & Palomar & $\ldots$ & $\ldots$ & $\ldots$ & $\ldots$ & $\ldots$ \\
\hline & 2004 Oct 04 & Palomar & $\ldots$ & 14.3 & 16.4 & 18.3 & 18.5 \\
\hline HE 696 & 2003 Sep 20 & Palomar & $\ldots$ & 14.4 & 15.6 & 17.5 & 17.7 \\
\hline HE 699 & 2003 Sep 20 & Palomar & $\ldots$ & 14.1 & 16.2 & 17.5 & 17.7 \\
\hline HE 750 & 2003 Sep 20 & Palomar & $\ldots$ & 13.8 & 16.0 & 17.6 & 17.8 \\
\hline HE 767 & 2003 Sep 20 & Palomar & $\ldots$ & 13.9 & 16.1 & 17.5 & 17.8 \\
\hline HE 848 & 2003 Sep 20 & Palomar & $\ldots$ & 13.3 & 15.3 & 17.3 & 17.9 \\
\hline HE 935 & 2003 Sep 20 & Palomar & $\ldots$ & 13.2 & 15.4 & 17.1 & 17.7 \\
\hline & 2006 Dec 12 & Palomar & $\ldots$ & $\ldots$ & $\ldots$ & $\ldots$ & 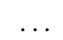 \\
\hline HE 1234 & 2003 Sep 20 & Palomar & $\ldots$ & 13.2 & 15.7 & 16.9 & 17.4 \\
\hline HD 22879 & 2004 Jun 04 & Palomar & ND1 & 9.7 & 11.3 & 14.4 & 14.9 \\
\hline Н II 102 & 2003 Sep 20 & Palomar & $\ldots$ & $\ldots$ & $\ldots$ & $\ldots$ & $\ldots$ \\
\hline & 2004 Oct 04 & Palomar & $\ldots$ & 13.3 & 15.6 & 17.1 & 17.3 \\
\hline & 2006 Jan 18 & Palomar & $\ldots$ & $\ldots$ & $\ldots$ & $\ldots$ & $\ldots$ \\
\hline H II 120 & 2003 Dec 10 & Palomar & $\ldots$ & 13.4 & 15.3 & 17.2 & 17.9 \\
\hline H II 152 & 2003 Sep 21 & Palomar & $\ldots$ & 13.5 & 15.4 & 16.4 & 16.7 \\
\hline H II 174 & 2003 Sep 21 & Palomar & $\ldots$ & 13.8 & 15.8 & 16.8 & 17.0 \\
\hline H II 173 & 2003 Sep 21 & Palomar & $\ldots$ & 13.0 & 14.9 & 16.2 & 16.6 \\
\hline Н II 250 & 2003 Dec 10 & Palomar & $\ldots$ & 13.3 & 15.9 & 17.3 & 18.0 \\
\hline Н II 314 & 2003 Dec 10 & Palomar & $\ldots$ & 13.2 & 15.1 & 17.0 & 17.6 \\
\hline 1RXS J034423.3+281224 & 2002 Nov 17 & Palomar & ND1 & $\ldots$ & $\ldots$ & $\ldots$ & $\ldots$ \\
\hline & 2004 Oct 05 & Palomar & ND1 & 11.5 & 12.0 & 15.8 & 16.3 \\
\hline Н II 514 & 2003 Dec 10 & Palomar & $\ldots$ & 13.3 & 15.2 & 16.9 & 17.4 \\
\hline Н II 571 & 2003 Dec 10 & Palomar & $\ldots$ & $\ldots$ & $\ldots$ & $\ldots$ & $\ldots$ \\
\hline & 2004 Oct 03 & Palomar & $\ldots$ & 13.8 & 16.0 & 17.7 & 17.8 \\
\hline H II 1015 & 2003 Dec 10 & Palomar & $\ldots$ & 13.2 & 15.2 & 17.0 & 17.6 \\
\hline H II 1101 & 2003 Dec 10 & Palomar & $\ldots$ & $\ldots$ & $\ldots$ & $\ldots$ & $\ldots$ \\
\hline & 2004 Oct 05 & Palomar & $\ldots$ & 13.2 & 15.4 & 17.3 & 17.7 \\
\hline H II 1182 & 2003 Dec 10 & Palomar & $\ldots$ & $\ldots$ & $\ldots$ & $\ldots$ & $\ldots$ \\
\hline & 2004 Oct 03 & Palomar & $\ldots$ & $\ldots$ & $\ldots$ & $\ldots$ & $\ldots$ \\
\hline & 2005 Jan 24 & Palomar & $\ldots$ & 13.8 & 15.8 & 17.5 & 18.0 \\
\hline H II 1776 & 2003 Dec 10 & Palomar & $\ldots$ & 13.2 & 14.4 & 16.0 & 16.3 \\
\hline H II 2106 & 2003 Dec 10 & Palomar & $\ldots$ & $\ldots$ & $\ldots$ & $\ldots$ & $\ldots$ \\
\hline & 2005 Jan 24 & Palomar & $\ldots$ & 13.3 & 16.7 & 18.1 & 18.2 \\
\hline RX J0348.9+0110 & 2003 Dec 10 & Palomar & $\ldots$ & 11.8 & 13.6 & 15.2 & 16.0 \\
\hline
\end{tabular}


Table 7

(Continued)

\begin{tabular}{|c|c|c|c|c|c|c|c|}
\hline \multirow[t]{2}{*}{ Star } & \multirow{2}{*}{$\begin{array}{l}\text { Date } \\
\text { (UT) }\end{array}$} & \multirow[t]{2}{*}{ Observatory } & \multirow[t]{2}{*}{ Optic $^{b}$} & \multicolumn{4}{|c|}{ Limiting $K_{S}$-Band Magnitude at ${ }^{\mathrm{a}}$} \\
\hline & & & & 0.5 & $1^{\prime \prime}$ & $2^{\prime \prime}$ & $5^{\prime \prime}$ \\
\hline & 2005 Jan 24 & Palomar & $\ldots$ & $\ldots$ & $\ldots$ & $\ldots$ & $\ldots$ \\
\hline \multirow[t]{2}{*}{ H II 2278} & 2003 Dec 10 & Palomar & $\ldots$ & $\ldots$ & $\ldots$ & $\ldots$ & $\ldots$ \\
\hline & 2005 Jan 24 & Palomar & $\ldots$ & 11.3 & 14.6 & 17.1 & 17.6 \\
\hline Н II 2506 & $2003 \operatorname{Dec} 10$ & Palomar & $\ldots$ & 12.8 & 15.0 & 17.1 & 17.8 \\
\hline H II 2644 & 2003 Dec 10 & Palomar & $\ldots$ & 13.6 & 15.7 & 17.2 & 17.7 \\
\hline Н II 2786 & 2003 Dec 10 & Palomar & $\ldots$ & 12.9 & 15.4 & 17.0 & 17.7 \\
\hline \multirow[t]{2}{*}{ Н II 2881} & 2003 Dec 10 & Palomar & $\ldots$ & $\ldots$ & $\ldots$ & $\ldots$ & $\ldots$ \\
\hline & 2005 Jan 24 & Palomar & $\ldots$ & 13.8 & 15.9 & 17.6 & 17.8 \\
\hline Н II 3097 & $2003 \operatorname{Dec} 10$ & Palomar & $\ldots$ & 13.6 & 15.5 & 17.4 & 17.9 \\
\hline Н II 3179 & 2005 Jan 24 & Palomar & $\ldots$ & 13.2 & 15.4 & 17.3 & 17.5 \\
\hline \multirow[t]{2}{*}{ HD 285281} & 2002 Feb 01 & Palomar & $\ldots$ & $\ldots$ & $\ldots$ & $\ldots$ & $\ldots$ \\
\hline & 2004 Feb 07 & Palomar & $\ldots$ & 12.7 & 12.7 & 15.5 & 17.8 \\
\hline \multirow[t]{2}{*}{ HD 284135} & 2002 Jan 31 & Palomar & $\ldots$ & 10.9 & 12.0 & 14.8 & 17.3 \\
\hline & 2004 Feb 07 & Palomar & ND1 & $\ldots$ & $\ldots$ & $\ldots$ & $\ldots$ \\
\hline \multirow[t]{2}{*}{ HD 281691} & 2002 Nov 18 & Palomar & $\ldots$ & $\ldots$ & $\ldots$ & $\ldots$ & $\ldots$ \\
\hline & 2004 Feb 07 & Palomar & $\ldots$ & 13.3 & 15.1 & 17.0 & 17.8 \\
\hline HD 26182 & 2003 Dec 10 & Palomar & $\ldots$ & 12.4 & 13.1 & 14.5 & 17.0 \\
\hline \multirow[t]{2}{*}{ HD 284266} & 2002 Jan 31 & Palomar & $\ldots$ & 12.5 & 12.8 & 15.8 & 17.8 \\
\hline & 2003 Dec 10 & Palomar & $\ldots$ & $\ldots$ & $\ldots$ & $\ldots$ & $\ldots$ \\
\hline \multirow[t]{2}{*}{ HD 26990} & 2003 Dec 10 & Palomar & ND1 & 9.6 & 12.0 & 14.0 & 14.9 \\
\hline & 2005 Jan 24 & Palomar & ND1 & $\ldots$ & & $\ldots$ & $\ldots$ \\
\hline HD 27466 & 2003 Dec 10 & Palomar & ND1 & 10.4 & 12.3 & 14.1 & 14.7 \\
\hline vB 39 & 2003 Jan 12 & Palomar & $\ldots$ & 9.9 & 12.4 & 14.5 & 15.6 \\
\hline vB 49 & 2003 Jan 12 & Palomar & $\ldots$ & 10.4 & 12.8 & 14.3 & 15.2 \\
\hline vB 52 & 2003 Jan 12 & Palomar & $\ldots$ & 10.6 & 12.8 & 12.8 & 16.4 \\
\hline & 2004 Oct 05 & Palomar & ND1 & $\ldots$ & $\ldots$ & $\ldots$ & $\ldots$ \\
\hline vB 176 & 2003 Dec 10 & Palomar & $\ldots$ & $\ldots$ & $\ldots$ & $\ldots$ & $\ldots$ \\
\hline & 2004 Oct 03 & Palomar & ND1 & 9.7 & 13.3 & 15.6 & 16.3 \\
\hline vB 63 & 2003 Jan 12 & Palomar & $\ldots$ & 10.8 & 13.1 & 14.9 & 16.5 \\
\hline vB 64 & 2004 Jun 04 & Palomar & ND1 & 10.9 & 12.9 & 14.9 & 16.4 \\
\hline vB 66 & 2002 Nov 17 & Palomar & Corona & 10.8 & 14.8 & 16.3 & 19.3 \\
\hline vB 73 & 2003 Jan 12 & Palomar & $\ldots$ & 10.7 & 13.0 & 14.3 & 15.0 \\
\hline vB 79 & 2004 Jun 04 & Palomar & ND1 & 11.3 & 13.5 & 15.3 & 17.3 \\
\hline vB 180 & 2003 Dec 10 & Palomar & $\ldots$ & 11.6 & 13.9 & 15.6 & 17.6 \\
\hline vB 88 & 2003 Dec 10 & Palomar & $\ldots$ & 10.8 & 12.9 & 14.7 & 16.7 \\
\hline 1RXS J043243.2-152003 & 2003 Jan 12 & Palomar & $\ldots$ & 12.8 & 14.9 & 16.2 & 17.2 \\
\hline vB 91 & 2003 Dec 10 & Palomar & $\ldots$ & $\ldots$ & $\ldots$ & $\ldots$ & $\ldots$ \\
\hline & 2004 Oct 03 & Palomar & ND1 & 12.4 & 13.6 & 16.7 & 17.0 \\
\hline vB 92 & 2004 Jun 04 & Palomar & ND1 & 11.2 & 13.5 & 15.2 & 16.8 \\
\hline vB 93 & 2004 Jun 04 & Palomar & ND1 & 11.5 & 13.5 & 15.5 & 17.3 \\
\hline vB 96 & 2003 Dec 10 & Palomar & $\ldots$ & 10.3 & 13.2 & 14.3 & 16.8 \\
\hline RX J0434.3+0226 & 2003 Jan 12 & Palomar & $\ldots$ & 13.9 & 16.1 & 15.3 & 17.5 \\
\hline vB 183 & 2004 Jun 04 & Palomar & ND1 & 11.7 & 14.0 & 15.8 & 17.3 \\
\hline vB 97 & 2004 Jun 04 & Palomar & ND1 & 10.8 & 12.8 & 14.6 & 16.7 \\
\hline vB 99 & 2004 Jun 04 & Palomar & ND1 & 11.5 & 13.5 & 15.6 & 17.2 \\
\hline vB 106 & 2003 Dec 10 & Palomar & $\ldots$ & $\ldots$ & $\ldots$ & $\ldots$ & $\ldots$ \\
\hline & 2004 Oct 05 & Palomar & Corona & 13.9 & 15.1 & 16.8 & 19.6 \\
\hline HD 282346 & 2002 Nov 18 & Palomar & $\ldots$ & 11.6 & 12.0 & 15.5 & 17.4 \\
\hline & 2004 Oct 04 & Palomar & ND1 & $\ldots$ & $\ldots$ & $\ldots$ & $\ldots$ \\
\hline vB 142 & 2002 Nov 17 & Palomar & Corona & $\ldots$ & $\ldots$ & $\ldots$ & $\ldots$ \\
\hline & 2003 Nov 10 & Keck & Corona & 14.3 & 16.8 & 18.6 & 19.6 \\
\hline & $2004 \mathrm{Feb} 07$ & Palomar & Corona & $\ldots$ & $\ldots$ & $\ldots$ & $\ldots$ \\
\hline vB 143 & 2004 Jun 04 & Palomar & ND1 & 10.8 & 12.2 & 13.2 & 13.4 \\
\hline HD 286264 & 2003 Dec 10 & Palomar & $\ldots$ & 11.8 & 13.6 & 15.5 & 17.0 \\
\hline HD 32850 & 2004 Jun 04 & Palomar & ND1 & 10.1 & 11.7 & 13.3 & 13.6 \\
\hline 1RXS J051111.1+281353 & 2002 Feb 28 & Palomar & $\ldots$ & 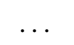 & $\ldots$ & . & $\ldots$ \\
\hline & 2003 Jan 14 & Palomar & $\ldots$ & 12.1 & 11.6 & 15.1 & 17.2 \\
\hline & 2003 Dec 10 & Palomar & $\ldots$ & $\ldots$ & $\ldots$ & $\ldots$ & $\ldots$ \\
\hline HD 36869 & 2003 Jan 14 & Palomar & Corona & 11.0 & 13.0 & 17.3 & 19.8 \\
\hline & 2004 Oct 05 & Palomar & ND1 & $\ldots$ & $\ldots$ & $\ldots$ & \\
\hline HD 37216 & 2004 Jun 04 & Palomar & ND1 & 10.5 & 12.3 & 13.7 & 13.9 \\
\hline HD 37006 & 2003 Jan 11 & Palomar & $\ldots$ & $\ldots$ & $\ldots$ & $\ldots$ & $\ldots$ \\
\hline & 2003 Dec 10 & Palomar & $\ldots$ & 11.1 & 12.8 & 14.5 & 16.6 \\
\hline HD 38529 & 2002 Nov 18 & Palomar & ND1 & 8.6 & 10.0 & 12.3 & 13.4 \\
\hline HD 61994 & 2002 Nov 18 & Palomar & ND1 & 9.6 & 11.5 & 13.1 & 13.6 \\
\hline
\end{tabular}


Table 7

(Continued)

\begin{tabular}{|c|c|c|c|c|c|c|c|}
\hline \multirow[t]{2}{*}{ Star } & \multirow{2}{*}{$\begin{array}{l}\text { Date } \\
\text { (UT) }\end{array}$} & \multirow[t]{2}{*}{ Observatory } & \multirow[t]{2}{*}{ Optic $^{\mathrm{b}}$} & \multicolumn{4}{|c|}{ Limiting $K_{S}$-Band Magnitude at ${ }^{\mathrm{a}}$} \\
\hline & & & & 0.5 & $1^{\prime \prime}$ & $2^{\prime \prime}$ & $5^{\prime \prime}$ \\
\hline HD 64324 & 2004 Jun 04 & Palomar & ND1 & 10.2 & 12.2 & 13.5 & 13.8 \\
\hline HD 66751 & 2002 Nov 18 & Palomar & ND1 & 9.5 & 11.4 & 13.3 & 13.8 \\
\hline \multirow[t]{3}{*}{ HD 69076} & 2002 Nov 18 & Palomar & ND1 & $\ldots$ & $\ldots$ & $\ldots$ & $\ldots$ \\
\hline & 2003 May 11 & Palomar & ND1 & $\ldots$ & $\ldots$ & $\ldots$ & $\ldots$ \\
\hline & $2004 \mathrm{Feb} 06$ & Palomar & $\ldots$ & 11.0 & 12.9 & 12.9 & 16.3 \\
\hline HD 71974 & 2002 Mar 03 & Palomar & $\ldots$ & 9.8 & 10.2 & 12.1 & 14.1 \\
\hline HD 72687 & 2003 Dec 09 & Palomar & ND1 & 11.3 & 13.4 & 15.0 & 17.0 \\
\hline HD 72760 & 2002 Nov 16 & Palomar & ND1 & 10.1 & 11.5 & 13.6 & 14.2 \\
\hline HD 73668 & 2002 May 11 & Palomar & Corona & 10.1 & 19.7 & 18.9 & 16.6 \\
\hline HIP 42491 & 2002 Nov 17 & Palomar & ND1 & 10.9 & 12.8 & 13.8 & 14.0 \\
\hline HD 75302 & 2004 Jun 04 & Palomar & ND1 & 10.2 & 12.2 & 13.9 & 14.1 \\
\hline HD 76218 & 2004 Jun 04 & Palomar & ND1 & 10.2 & 12.1 & 14.0 & 14.3 \\
\hline \multirow[t]{3}{*}{ HD 77407} & 2002 Jan 31 & Palomar & $\ldots$ & 8.8 & 10.8 & 12.2 & 15.3 \\
\hline & 2003 Jan 13 & Palomar & ND1 & $\ldots$ & $\ldots$ & $\ldots$ & $\ldots$ \\
\hline & 2003 May 10 & Palomar & ND1 & $\ldots$ & $\ldots$ & $\ldots$ & $\ldots$ \\
\hline HD 78899 & 2003 Dec 09 & Palomar & ND1 & 10.3 & 12.0 & 14.4 & 15.2 \\
\hline HD 80606 & 2003 May 10 & Palomar & ND1 & 12.2 & 13.5 & 15.3 & 15.6 \\
\hline HD 85301 & 2004 Jun 04 & Palomar & ND1 & 10.5 & 12.3 & 13.9 & 14.2 \\
\hline HD 88638 & 2002 Jan 31 & Palomar & Corona & 10.3 & 13.6 & 14.5 & 18.0 \\
\hline \multirow[t]{3}{*}{ HD 91962} & 2002 Mar 02 & Palomar & $\ldots$ & 9.5 & 10.8 & 12.0 & 16.3 \\
\hline & 2003 May 10 & Palomar & ND1 & $\ldots$ & $\ldots$ & $\ldots$ & $\ldots$ \\
\hline & 2004 Feb 05 & Palomar & ND1 & $\ldots$ & $\ldots$ & $\ldots$ & $\ldots$ \\
\hline HD 92788 & 2004 Jun 04 & Palomar & ND1 & 9.9 & 11.7 & 13.9 & 14.6 \\
\hline HD 98553 & 2003 Jan 11 & Palomar & $\ldots$ & 10.1 & 11.5 & 13.5 & 16.3 \\
\hline \multirow[t]{4}{*}{ HD 99565} & 2003 Jan 11 & Palomar & ND1 & $\ldots$ & $\ldots$ & $\ldots$ & $\ldots$ \\
\hline & 2003 May 11 & Palomar & ND1 & $\ldots$ & $\ldots$ & $\ldots$ & $\ldots$ \\
\hline & 2003 Jul 16 & Palomar & $\ldots$ & 9.1 & 9.9 & 12.3 & 15.8 \\
\hline & $2004 \mathrm{Feb} 05$ & Palomar & ND1 & 9.1 & 9.9 & 12.3 & 15.8 \\
\hline HD 100167 & 2002 Mar 03 & Palomar & Corona & 9.1 & 12.6 & 14.1 & 17.8 \\
\hline HD 101959 & 2002 Feb 28 & Palomar & $\ldots$ & 8.9 & 10.6 & 13.7 & 15.4 \\
\hline HD 102071 & $2002 \mathrm{Feb} 28$ & Palomar & $\ldots$ & 9.3 & 11.1 & 14.3 & 15.4 \\
\hline HD 103432 & 2004 Jun 04 & Palomar & ND1 & 10.2 & 12.3 & 14.0 & 14.2 \\
\hline HD 105631 & 2004 Jun 04 & Palomar & ND1 & 9.9 & 11.6 & 13.8 & 15.0 \\
\hline HD 106156 & 2004 Jun 04 & Palomar & ND1 & 10.4 & 12.0 & 13.7 & 14.6 \\
\hline HD 106252 & 2004 Jun 04 & Palomar & ND1 & 9.4 & 11.6 & 13.6 & 14.0 \\
\hline \multirow[t]{4}{*}{ HD 108799} & 2003 May 10 & Palomar & ND1 & 9.8 & 11.2 & 13.3 & 14.5 \\
\hline & $2003 \mathrm{Jul} 16$ & Palomar & ND1 & $\ldots$ & $\ldots$ & $\ldots$ & $\ldots$ \\
\hline & 2004 Feb 05 & Palomar & ND1 & $\ldots$ & $\ldots$ & $\ldots$ & $\ldots$ \\
\hline & 2004 Jun 26 & Palomar & ND1 & $\ldots$ & $\ldots$ & $\ldots$ & $\ldots$ \\
\hline \multirow[t]{6}{*}{ HD 108944} & 2002 Mar 03 & Palomar & $\ldots$ & $\ldots$ & $\ldots$ & $\ldots$ & $\ldots$ \\
\hline & 2002 Jun 21 & Palomar & $\ldots$ & 10.4 & 12.3 & 15.2 & 17.6 \\
\hline & 2003 Jan 13 & Palomar & ND1 & $\ldots$ & $\ldots$ & $\ldots$ & $\ldots$ \\
\hline & 2003 May 11 & Palomar & ND1 & $\ldots$ & $\ldots$ & $\ldots$ & $\ldots$ \\
\hline & 2004 Feb 05 & Palomar & ND1 & $\ldots$ & $\ldots$ & $\ldots$ & $\ldots$ \\
\hline & 2004 Jun 27 & Palomar & ND1 & $\ldots$ & $\ldots$ & $\ldots$ & $\ldots$ \\
\hline \multirow[t]{5}{*}{ HD 112196} & $2002 \mathrm{Feb} 01$ & Palomar & $\ldots$ & $\ldots$ & $\ldots$ & $\ldots$ & $\ldots$ \\
\hline & 2003 Jan 13 & Palomar & ND1 & $\ldots$ & $\ldots$ & $\ldots$ & $\ldots$ \\
\hline & 2003 May 11 & Palomar & ND1 & $\ldots$ & $\ldots$ & $\ldots$ & $\ldots$ \\
\hline & 2004 Feb 05 & Palomar & ND1 & $\ldots$ & $\ldots$ & $\ldots$ & $\ldots$ \\
\hline & 2004 Jun 26 & Palomar & ND1 & 10.5 & 12.1 & 13.4 & 14.5 \\
\hline HD 115043 & 2003 Dec 09 & Palomar & ND1 & $\ldots$ & $\ldots$ & $\ldots$ & $\ldots$ \\
\hline & 2004 Jun 27 & Palomar & ND1 & 10.2 & 11.8 & 14.6 & 15.7 \\
\hline HD 121320 & 2004 Jun 04 & Palomar & ND1 & 10.4 & 12.3 & 14.0 & 14.4 \\
\hline HD 122652 & 2004 Jun 04 & Palomar & ND1 & 10.0 & 11.9 & 14.0 & 15.1 \\
\hline HD 129333 & 2003 Jan 11 & Palomar & ND1 & $\ldots$ & $\ldots$ & $\ldots$ & $\ldots$ \\
\hline & 2003 Jan 12 & Palomar & ND1 & $\ldots$ & $\ldots$ & $\ldots$ & $\ldots$ \\
\hline & 2003 May 13 & Palomar & $\ldots$ & 10.5 & 11.7 & 14.3 & 15.2 \\
\hline & $2004 \mathrm{Feb} 05$ & Palomar & ND1 & $\ldots$ & $\ldots$ & $\ldots$ & $\ldots$ \\
\hline HD 133295 & $2002 \mathrm{Feb} 28$ & Palomar & $\ldots$ & 9.2 & 10.8 & 13.8 & 16.1 \\
\hline HD 134319 & 2002 Mar 02 & Palomar & $\ldots$ & $\ldots$ & $\ldots$ & $\ldots$ & $\ldots$ \\
\hline & 2003 Jan 14 & Palomar & ND1 & $\ldots$ & $\ldots$ & $\ldots$ & $\ldots$ \\
\hline & 2004 Jun 27 & Palomar & ND1 & 11.7 & 13.2 & 15.3 & 15.6 \\
\hline HD 135363 & $2002 \mathrm{Feb} 01$ & Palomar & $\ldots$ & $\ldots$ & $\ldots$ & $\ldots$ & $\ldots$ \\
\hline & 2003 Jan 14 & Palomar & ND1 & $\ldots$ & $\ldots$ & $\ldots$ & $\ldots$ \\
\hline & 2004 Jun 27 & Palomar & ND1 & 9.5 & 12.9 & 15.2 & 15.8 \\
\hline
\end{tabular}


Table 7

(Continued)

\begin{tabular}{|c|c|c|c|c|c|c|c|}
\hline \multirow[t]{2}{*}{ Star } & \multirow{2}{*}{$\begin{array}{l}\text { Date } \\
\text { (UT) }\end{array}$} & \multirow[t]{2}{*}{ Observatory } & \multirow[t]{2}{*}{ Optic $^{b}$} & \multicolumn{4}{|c|}{ Limiting $K_{S}$-Band Magnitude at ${ }^{\mathrm{a}}$} \\
\hline & & & & 0.5 & $1^{\prime \prime}$ & $2^{\prime \prime}$ & $5^{\prime \prime}$ \\
\hline HD 136923 & 2004 Jun 04 & Palomar & ND1 & 9.3 & 11.3 & 13.6 & 15.1 \\
\hline HD 138004 & 2004 Jun 04 & Palomar & ND1 & 9.9 & 11.9 & 14.2 & 15.1 \\
\hline \multirow[t]{2}{*}{ RX J1541.1-2656 } & 2003 Jul 15 & Palomar & $\ldots$ & 13.4 & 15.7 & 17.3 & 17.6 \\
\hline & 2004 Jun 27 & Palomar & $\ldots$ & $\ldots$ & $\ldots$ & $\ldots$ & \\
\hline HD 142229 & 2004 Jun 04 & Palomar & ND1 & 10.9 & 12.5 & 15.2 & 16.2 \\
\hline RX J1600.6-2159 & 2003 Jul 15 & Palomar & $\ldots$ & 13.0 & 14.9 & 17.0 & 17.7 \\
\hline PZ99 J160814.7-190833 & 2002 Aug 31 & Palomar & $\ldots$ & 12.2 & 14.6 & 16.7 & 17.5 \\
\hline HD 145229 & 2003 May 10 & Palomar & ND1 & 10.9 & 12.2 & 15.1 & 15.7 \\
\hline \multirow[t]{2}{*}{ PZ99 J161329.3-231106 } & 2003 May 10 & Palomar & $\ldots$ & $\ldots$ & $\ldots$ & $\ldots$ & $\ldots$ \\
\hline & 2004 Jun 26 & Palomar & $\ldots$ & 13.5 & 15.4 & 15.3 & 18.0 \\
\hline HD 150706 & 2003 May 11 & Palomar & ND1 & 9.9 & 11.2 & 13.2 & 14.6 \\
\hline \multirow[t]{2}{*}{ HD 150554} & 2003 May 10 & Palomar & ND1 & 11.1 & 12.4 & 14.7 & 14.9 \\
\hline & 2003 Jul 16 & Palomar & ND1 & $\ldots$ & $\ldots$ & $\ldots$ & $\ldots$ \\
\hline \multirow[t]{3}{*}{ HD 152555} & 2002 Aug 31 & Palomar & ND1 & $\ldots$ & $\ldots$ & $\ldots$ & $\ldots$ \\
\hline & 2003 Jul 16 & Palomar & ND1 & $\ldots$ & $\ldots$ & $\ldots$ & $\ldots$ \\
\hline & 2004 Jun 26 & Palomar & ND1 & 11.1 & 12.8 & 15.1 & 15.4 \\
\hline HD 153458 & 2004 Jun 04 & Palomar & ND1 & 12.6 & 12.6 & 14.9 & 15.3 \\
\hline HD 154417 & 2003 May 10 & Palomar & ND1 & 9.4 & 10.8 & 13.8 & 14.9 \\
\hline HD 155902 & 2003 Sep 21 & Palomar & ND1 & 10.0 & 11.6 & 13.9 & 14.6 \\
\hline \multirow[t]{2}{*}{ HD 157664} & 2003 May 10 & Palomar & Corona & 10.0 & 15.5 & 17.2 & 20.0 \\
\hline & 2004 Oct 04 & Palomar & ND1 & $\ldots$ & $\ldots$ & $\ldots$ & \\
\hline HD 159222 & 2003 May 11 & Palomar & ND1 & 9.2 & 11.1 & 13.6 & 14.3 \\
\hline HD 161897 & 2003 May 13 & Palomar & ND1 & 10.0 & 11.7 & 13.5 & 13.9 \\
\hline \multirow[t]{4}{*}{ HD 166435} & 2002 Jun 23 & Palomar & Corona & $\ldots$ & $\ldots$ & $\ldots$ & $\ldots$ \\
\hline & 2002 Aug 30 & Palomar & Corona & 10.0 & 14.3 & 16.2 & 18.2 \\
\hline & 2003 May 16 & Keck & Corona & $\ldots$ & $\ldots$ & $\ldots$ & $\ldots$ \\
\hline & 2004 Jun 26 & Palomar & Corona & $\ldots$ & $\ldots$ & $\ldots$ & $\ldots$ \\
\hline \multirow[t]{2}{*}{ HD 167389} & 2003 May 13 & Palomar & ND1 & 10.7 & 12.3 & 14.9 & 15.6 \\
\hline & 2004 Jun 04 & Palomar & ND1 & 10.7 & 12.3 & 14.9 & 15.6 \\
\hline HD 175742 & 2004 Jun 28 & Palomar & Corona & 10.7 & 15.3 & 16.9 & 20.5 \\
\hline HD 179949 & 2003 Sep 20 & Palomar & ND1 & 9.7 & 11.3 & 13.7 & 14.2 \\
\hline HD 187897 & 2003 Sep 20 & Palomar & ND1 & 10.2 & 12.0 & 13.9 & 14.2 \\
\hline HD 190228 & 2003 Sep 20 & Palomar & ND1 & 10.1 & 11.7 & 14.0 & 14.3 \\
\hline \multirow[t]{2}{*}{ HD 193216} & 2003 Jul 16 & Palomar & Corona & $\ldots$ & $\ldots$ & $\ldots$ & $\ldots$ \\
\hline & 2004 Jun 27 & Palomar & Corona & 10.7 & 15.4 & 17.2 & 20.1 \\
\hline HD 193017 & 2003 Sep 21 & Palomar & ND1 & 10.6 & 12.3 & 14.4 & 15.0 \\
\hline HD 195034 & 2003 Sep 20 & Palomar & ND1 & 10.3 & 11.9 & 14.5 & 15.0 \\
\hline \multirow[t]{4}{*}{ HD 199143} & 2002 Jun 23 & Palomar & ND1 & $\ldots$ & $\ldots$ & $\ldots$ & $\ldots$ \\
\hline & 2003 Sep 21 & Palomar & ND1 & $\ldots$ & $\ldots$ & $\ldots$ & $\ldots$ \\
\hline & 2004 Jun 26 & Palomar & ND1 & $\ldots$ & $\ldots$ & $\ldots$ & $\ldots$ \\
\hline & 2004 Oct 05 & Palomar & ND1 & 10.7 & 12.5 & 13.4 & 15.4 \\
\hline HD 199598 & 2003 Sep 20 & Palomar & ND1 & 10.1 & 11.8 & 14.4 & 14.9 \\
\hline HD 201219 & 2003 Sep 21 & Palomar & ND1 & 10.6 & 12.8 & 14.8 & 15.3 \\
\hline HD 202108 & 2003 Sep 20 & Palomar & ND1 & 10.4 & 12.1 & 14.4 & 14.9 \\
\hline HD 201989 & 2003 Jul 16 & Palomar & ND1 & 10.2 & 11.9 & 13.8 & 14.0 \\
\hline HD 204277 & 2004 Jun 04 & Palomar & ND1 & 9.9 & 11.8 & 14.0 & 14.4 \\
\hline HIP 106335 & 2004 Jun 28 & Palomar & Corona & 10.2 & 15.8 & 17.4 & 20.0 \\
\hline HD 205905 & $2003 \mathrm{Jul} 16$ & Palomar & $\ldots$ & 9.7 & 11.9 & 14.2 & 16.6 \\
\hline HD 206374 & 2003 Jul 16 & Palomar & $\ldots$ & 12.5 & 14.3 & 16.5 & 18.0 \\
\hline HD 212291 & 2004 Jun 04 & Palomar & ND1 & 10.4 & 12.5 & 14.3 & 14.9 \\
\hline HD 216275 & 2003 May 10 & Palomar & ND1 & 10.7 & 11.8 & 14.5 & 14.9 \\
\hline \multirow[t]{3}{*}{ RX J2312.0+2245 } & 2002 Aug 30 & Palomar & ND1 & $\ldots$ & $\ldots$ & $\ldots$ & $\ldots$ \\
\hline & 2003 Sep 20 & Palomar & $\ldots$ & 13.0 & 15.3 & 17.1 & 17.6 \\
\hline & 2004 Oct 05 & Palomar & $\ldots$ & $\ldots$ & $\ldots$ & $\ldots$ & $\ldots$ \\
\hline RX J2313.0+2345 & 2002 Aug 30 & Palomar & $\ldots$ & 13.2 & 15.6 & 14.0 & 18.5 \\
\hline \multirow[t]{2}{*}{ HD 221613} & 2002 Nov 18 & Palomar & ND1 & $\ldots$ & $\ldots$ & $\ldots$ & $\ldots$ \\
\hline & 2003 Sep 21 & Palomar & ND1 & 9.7 & 12.2 & 14.0 & 14.3 \\
\hline
\end{tabular}

Notes.

a The limiting magnitude corresponds to the apparent $K_{S}$-band magnitude of the faintest detectable point source at the given angular separation, and is quoted only for the epoch of the deepest observation.

b "Corona" entries in the column "Optic" indicate that a coronagraph has been used. Likewise, "ND1" indicates that the ND 1\% filter was used at Palomar.

(This table is also available in a machine-readable form in the online journal.) 
Table 8

Candidate Companions in the Deep Sample

\begin{tabular}{|c|c|c|c|c|c|c|c|c|c|c|}
\hline Primary Star & $N_{C}$ & $\begin{array}{c}\rho \\
(\operatorname{arcsec})\end{array}$ & $\begin{array}{c}\theta \\
(\mathrm{deg})\end{array}$ & $\begin{array}{c}\Delta K_{S} \\
(\mathrm{mag})\end{array}$ & $\begin{array}{c}K_{S} \\
(\mathrm{mag}) \\
\end{array}$ & $\begin{array}{c}J-K_{S} \\
(\mathrm{mag})\end{array}$ & $\begin{array}{c}t_{0} \\
\text { (UT Date) }\end{array}$ & Tel. & Assoc. & Ref. \\
\hline QT And & 1 & $7.696 \pm 0.019$ & $239.56 \pm 0.22$ & $11.56 \pm 0.22$ & $18.91 \pm 0.22$ & $1.57 \pm 0.31$ & 2002 Aug 29 & $\mathrm{P}$ & no(a) & \\
\hline HD 15526 & 1 & $0.077 \pm 0.004$ & $177.96 \pm 0.75$ & $0.00 \pm 0.05$ & $8.76 \pm 0.06$ & $0.61 \pm 0.08$ & 2003 Sep 20 & $\mathrm{P}$ & yes $(c)$ & \\
\hline 1RXS J025216.9+361658 & 1 & $5.811 \pm 0.020$ & $10.63 \pm 0.41$ & $10.26 \pm 0.09$ & $17.86 \pm 0.09$ & $\ldots$ & 2002 Nov 18 & $\mathrm{P}$ & no(a) & \\
\hline RX J0258.4+2947 & 1 & $0.086 \pm 0.011$ & $220.82 \pm 4.14$ & $0.60 \pm 0.30$ & $10.15 \pm 0.30$ & $0.58 \pm 0.42$ & 2002 Feb 28 & $\mathrm{P}$ & yes(c) & \\
\hline HD 19668 & 1 & $6.565 \pm 0.020$ & $148.98 \pm 0.19$ & $10.58 \pm 0.03$ & $17.28 \pm 0.04$ & $\ldots$ & 2002 Aug 27 & $\mathrm{P}$ & no(a) & \\
\hline \multirow[t]{3}{*}{ V525 Per } & 1 & $4.135 \pm 0.026$ & $83.86 \pm 0.23$ & $7.57 \pm 0.30$ & $16.93 \pm 0.30$ & $\ldots$ & 2003 Sep 20 & $\mathrm{P}$ & no(a) & \\
\hline & 2 & $12.452 \pm 0.021$ & $64.09 \pm 0.13$ & $7.23 \pm 0.32$ & $16.59 \pm 0.32$ & $\ldots$ & 2004 Oct 03 & $\mathrm{P}$ & $?$ & \\
\hline & 3 & $10.680 \pm 0.043$ & $126.00 \pm 0.20$ & $9.98 \pm 0.20$ & $19.34 \pm 0.20$ & $\ldots$ & 2004 Oct 08 & $\mathrm{~K}$ & $?$ & \\
\hline \multirow[t]{2}{*}{ 1RXS J031907.4+393418 } & 1 & $7.656 \pm 0.030$ & $286.56 \pm 0.25$ & $8.77 \pm 0.09$ & $18.26 \pm 0.09$ & $0.87 \pm 0.24$ & 2002 Aug 29 & $\mathrm{P}$ & no(a) & \\
\hline & 2 & $10.157 \pm 0.024$ & $333.52 \pm 0.18$ & $9.69 \pm 0.09$ & $19.18 \pm 0.09$ & $1.14 \pm 0.24$ & 2002 Aug 29 & $\mathrm{P}$ & no(a) & \\
\hline \multirow[t]{5}{*}{ HE 622} & 1 & $7.275 \pm 0.017$ & $48.24 \pm 0.18$ & $6.38 \pm 0.22$ & $15.97 \pm 0.22$ & $0.66 \pm 0.31$ & 2003 Sep 20 & $\mathrm{P}$ & $?$ & \\
\hline & 2 & $9.756 \pm 0.024$ & $311.79 \pm 0.17$ & $6.51 \pm 0.22$ & $16.10 \pm 0.22$ & $0.83 \pm 0.31$ & 2003 Sep 20 & $\mathrm{P}$ & $?$ & \\
\hline & 3 & $12.478 \pm 0.021$ & $107.92 \pm 0.12$ & $8.76 \pm 0.22$ & $18.35 \pm 0.22$ & $1.03 \pm 0.31$ & 2004 Oct 08 & $\mathrm{~K}$ & $?$ & \\
\hline & 4 & $12.368 \pm 0.023$ & $109.57 \pm 0.12$ & $9.58 \pm 0.22$ & $19.17 \pm 0.22$ & $0.98 \pm 0.31$ & 2004 Oct 08 & $\mathrm{~K}$ & $?$ & \\
\hline & 5 & $10.436 \pm 0.017$ & $224.37 \pm 0.12$ & $8.10 \pm 0.22$ & $17.69 \pm 0.22$ & $1.12 \pm 0.31$ & 2004 Oct 08 & $\mathrm{~K}$ & $?$ & \\
\hline RX J0329.1+0118 & 1 & $3.761 \pm 0.004$ & $303.35 \pm 0.09$ & $3.62 \pm 0.08$ & $12.82 \pm 0.08$ & $0.90 \pm 0.14$ & 2003 Sep 21 & $\mathrm{P}$ & yes $(c)$ & MH04 \\
\hline \multirow[t]{5}{*}{ HE 1101} & 1 & $5.828 \pm 0.025$ & $323.66 \pm 0.25$ & $6.58 \pm 0.09$ & $15.89 \pm 0.09$ & $0.34 \pm 0.13$ & 2003 Sep 20 & $\mathrm{P}$ & no(a) & \\
\hline & 2 & $5.911 \pm 0.010$ & $276.86 \pm 0.12$ & $8.25 \pm 0.09$ & $17.56 \pm 0.09$ & $0.14 \pm 0.13$ & 2004 Oct 07 & $\mathrm{~K}$ & no(a) & \\
\hline & 3 & $5.316 \pm 0.009$ & $247.23 \pm 0.12$ & $9.13 \pm 0.09$ & $18.44 \pm 0.09$ & $0.53 \pm 0.13$ & 2004 Oct 07 & $\mathrm{~K}$ & no(a) & \\
\hline & 4 & $10.100 \pm 0.017$ & $113.32 \pm 0.12$ & $9.63 \pm 0.09$ & $18.94 \pm 0.09$ & $0.61 \pm 0.13$ & 2004 Oct 07 & $\mathrm{~K}$ & no(a) & \\
\hline & 5 & $2.173 \pm 0.006$ & $29.19 \pm 0.14$ & $10.11 \pm 0.09$ & $19.42 \pm 0.09$ & $1.15 \pm 0.17$ & 2004 Oct 07 & $\mathrm{~K}$ & no(a) & \\
\hline \multirow[t]{3}{*}{ HD 22179} & 1 & $6.536 \pm 0.029$ & $236.26 \pm 0.24$ & $8.82 \pm 0.10$ & $16.24 \pm 0.10$ & $\ldots$ & 2002 Nov 09 & $\mathrm{P}$ & no(a) & \\
\hline & 2 & $6.616 \pm 0.029$ & $235.44 \pm 0.23$ & $9.30 \pm 0.11$ & $16.73 \pm 0.11$ & $\ldots$ & 2002 Nov 16 & $\mathrm{P}$ & no(a) & \\
\hline & 3 & $9.200 \pm 0.027$ & $179.64 \pm 0.23$ & $10.20 \pm 0.12$ & $17.62 \pm 0.12$ & $\ldots$ & 2002 Nov 09 & $\mathrm{P}$ & no(a) & \\
\hline \multirow[t]{2}{*}{ Н II 120} & 1 & $3.549 \pm 0.008$ & $119.15 \pm 0.14$ & $5.75 \pm 0.21$ & $14.85 \pm 0.21$ & $1.21 \pm 0.25$ & 2003 Sep 20 & $\mathrm{P}$ & no(a) & \\
\hline & 2 & $10.633 \pm 0.023$ & $70.53 \pm 0.13$ & $5.43 \pm 0.15$ & $14.53 \pm 0.15$ & $1.22 \pm 0.20$ & 2003 Sep 20 & $\mathrm{P}$ & no(a) & \\
\hline \multirow[t]{2}{*}{ RX J0354.4+0535 } & 1 & $11.128 \pm 0.035$ & $225.82 \pm 0.18$ & $7.27 \pm 0.10$ & $15.94 \pm 0.10$ & $0.62 \pm 0.18$ & $2003 \operatorname{Jan} 13$ & $\mathrm{P}$ & $\operatorname{no}(\mathrm{c})$ & \\
\hline & 2 & $0.205 \pm 0.004$ & $357.44 \pm 0.92$ & $2.10 \pm 0.20$ & $10.92 \pm 0.20$ & $0.97 \pm 0.28$ & 2004 Feb 07 & $\mathrm{P}$ & yes $(c)$ & \\
\hline \multirow[t]{2}{*}{ RX J0357.3+1258 } & 1 & $10.086 \pm 0.025$ & $115.72 \pm 0.19$ & $6.56 \pm 0.08$ & $15.54 \pm 0.08$ & $0.64 \pm 0.22$ & 2003 Jan 11 & $\mathrm{P}$ & no(a) & \\
\hline & 2 & $3.831 \pm 0.026$ & $338.31 \pm 0.26$ & $10.50 \pm 0.10$ & $19.48 \pm 0.10$ & $\ldots$ & 2003 Jan 11 & $\mathrm{P}$ & $?$ & \\
\hline \multirow[t]{2}{*}{ HD 286179} & 1 & $10.124 \pm 0.024$ & $237.40 \pm 0.19$ & $7.20 \pm 0.20$ & $15.66 \pm 0.20$ & $\ldots$ & 2002 Jan 31 & $\mathrm{P}$ & no(a) & \\
\hline & 2 & $3.406 \pm 0.009$ & $194.68 \pm 0.22$ & $10.72 \pm 0.18$ & $19.18 \pm 0.18$ & $\ldots$ & 2004 Oct 07 & $\mathrm{~K}$ & no(e) & \\
\hline \multirow[t]{7}{*}{ HD 31950} & 1 & $2.596 \pm 0.007$ & $264.22 \pm 0.18$ & $4.13 \pm 0.04$ & $12.51 \pm 0.05$ & $0.67 \pm 0.07$ & 2002 Nov 16 & $\mathrm{P}$ & yes(a) & \\
\hline & 2 & $3.106 \pm 0.007$ & $137.92 \pm 0.18$ & $3.70 \pm 0.04$ & $12.08 \pm 0.05$ & $0.49 \pm 0.07$ & 2002 Nov 16 & $\mathrm{P}$ & $\operatorname{no}(\mathrm{c})$ & \\
\hline & 3 & $6.925 \pm 0.016$ & $146.81 \pm 0.18$ & $6.35 \pm 0.04$ & $14.73 \pm 0.05$ & $0.89 \pm 0.07$ & 2002 Nov 16 & $\mathrm{P}$ & $?$ & \\
\hline & 4 & $3.117 \pm 0.015$ & $327.86 \pm 0.35$ & $8.53 \pm 0.05$ & $16.91 \pm 0.06$ & $0.31 \pm 0.08$ & 2002 Nov 16 & $\mathrm{P}$ & $\operatorname{no}(\mathrm{c})$ & \\
\hline & 5 & $10.013 \pm 0.027$ & $351.17 \pm 0.16$ & $9.91 \pm 0.09$ & $18.29 \pm 0.09$ & $\ldots$ & 2002 Nov 16 & $\mathrm{P}$ & $?$ & \\
\hline & 6 & $6.528 \pm 0.020$ & $28.55 \pm 0.14$ & $10.73 \pm 0.11$ & $19.11 \pm 0.11$ & $\ldots$ & 2002 Nov 16 & $\mathrm{P}$ & $?$ & \\
\hline & 7 & $6.313 \pm 0.019$ & $248.03 \pm 0.20$ & $10.36 \pm 0.08$ & $18.74 \pm 0.08$ & $\ldots$ & 2002 Nov 16 & $\mathrm{P}$ & $?$ & \\
\hline \multirow[t]{2}{*}{ 1RXS J053650.0+133756 } & 1 & $1.839 \pm 0.018$ & $37.26 \pm 0.54$ & $8.88 \pm 0.30$ & $16.95 \pm 0.30$ & $\ldots$ & 2002 Feb 28 & $\mathrm{P}$ & no(a) & \\
\hline & 2 & $12.096 \pm 0.027$ & $212.16 \pm 0.17$ & $8.10 \pm 0.10$ & $16.17 \pm 0.10$ & $\ldots$ & 2002 Feb 28 & $\mathrm{P}$ & no(a) & \\
\hline \multirow[t]{5}{*}{ HD 245567} & 1 & $0.348 \pm 0.002$ & $330.66 \pm 0.23$ & $1.79 \pm 0.04$ & $9.57 \pm 0.04$ & $0.52 \pm 0.08$ & 2002 Nov 16 & $\mathrm{P}$ & yes $(c)$ & \\
\hline & 2 & $3.185 \pm 0.007$ & $198.88 \pm 0.17$ & $6.44 \pm 0.24$ & $14.03 \pm 0.24$ & $0.54 \pm 0.34$ & 2002 Nov 16 & $\mathrm{P}$ & no(a) & \\
\hline & 3 & $6.748 \pm 0.024$ & $316.18 \pm 0.22$ & $8.28 \pm 0.24$ & $15.87 \pm 0.24$ & $0.97 \pm 0.34$ & 2002 Nov 16 & $\mathrm{P}$ & no(a) & \\
\hline & 4 & $10.927 \pm 0.024$ & $315.63 \pm 0.17$ & $6.21 \pm 0.24$ & $13.80 \pm 0.24$ & $0.55 \pm 0.34$ & 2002 Nov 16 & $\mathrm{P}$ & no(a) & \\
\hline & 5 & $2.724 \pm 0.007$ & $21.87 \pm 0.13$ & $11.55 \pm 0.24$ & $19.14 \pm 0.24$ & $\ldots$ & 2003 Nov 09 & $\mathrm{~K}$ & no(a) & \\
\hline SAO 150676 & 1 & $8.375 \pm 0.029$ & $351.31 \pm 0.14$ & $9.30 \pm 0.20$ & $16.77 \pm 0.20$ & $\ldots$ & 2002 Nov 17 & $\mathrm{P}$ & no(a) & \\
\hline HD 49197 & 1 & $6.952 \pm 0.016$ & $345.82 \pm 0.18$ & $6.75 \pm 0.06$ & $12.82 \pm 0.06$ & $0.15 \pm 0.12$ & 2002 Feb 28 & $\mathrm{P}$ & no(a) & \\
\hline & 2 & $0.948 \pm 0.032$ & $77.50 \pm 1.03$ & $8.22 \pm 0.14$ & $14.29 \pm 0.14$ & $1.63 \pm 1.21$ & 2002 Feb 28 & $\mathrm{P}$ & yes(a) & \\
\hline RE J0723+20 & 1 & $8.196 \pm 0.013$ & $80.86 \pm 0.03$ & $7.80 \pm 0.20$ & $14.68 \pm 0.20$ & $0.16 \pm 0.22$ & 2002 Feb 28 & $\mathrm{P}$ & no(a) & \\
\hline & 2 & $5.532 \pm 0.013$ & $329.36 \pm 0.09$ & $8.40 \pm 0.20$ & $15.28 \pm 0.20$ & $1.06 \pm 0.22$ & 2002 Feb 28 & $\mathrm{P}$ & no(a) & \\
\hline HD 60737 & 1 & $7.657 \pm 0.029$ & $127.25 \pm 0.18$ & $9.40 \pm 0.20$ & $15.65 \pm 0.20$ & $\ldots$ & 2002 Jan 31 & $\mathrm{P}$ & no(a) & \\
\hline HD 82443 & 1 & $5.459 \pm 0.010$ & $190.30 \pm 0.23$ & $11.77 \pm 0.14$ & $16.89 \pm 0.14$ & $\ldots$ & 2004 Feb 07 & $\mathrm{P}$ & $?$ & \\
\hline & 2 & $8.154 \pm 0.020$ & $98.76 \pm 0.15$ & $12.59 \pm 0.21$ & $17.71 \pm 0.21$ & $\cdots$ & 2004 Feb 07 & $\mathrm{P}$ & $?$ & \\
\hline & 3 & $7.142 \pm 0.027$ & $253.71 \pm 0.23$ & $13.84 \pm 0.30$ & $18.96 \pm 0.30$ & $\cdots$ & 2004 Feb 07 & $\mathrm{P}$ & $?$ & \\
\hline SAO 178272 & 1 & $10.082 \pm 0.032$ & $356.64 \pm 0.18$ & $9.67 \pm 0.15$ & $17.06 \pm 0.15$ & $\ldots$ & $2003 \operatorname{Jan} 13$ & $\mathrm{P}$ & $?$ & \\
\hline & 2 & $8.184 \pm 0.046$ & $274.53 \pm 0.15$ & $10.75 \pm 0.22$ & $18.14 \pm 0.22$ & $\ldots$ & 2003 Jan 13 & $\mathrm{P}$ & $?$ & \\
\hline HD 90905 & 1 & $5.816 \pm 0.027$ & $191.77 \pm 0.23$ & $11.30 \pm 0.10$ & $16.82 \pm 0.10$ & $\ldots$ & 2002 Feb 01 & $\mathrm{P}$ & no(a) & \\
\hline & 2 & $12.446 \pm 0.031$ & $176.73 \pm 0.13$ & $13.49 \pm 0.19$ & $19.01 \pm 0.19$ & $\ldots$ & 2004 Jun 05 & $\mathrm{~K}$ & no(e) & \\
\hline HD 91782 & 1 & $1.002 \pm 0.008$ & $33.67 \pm 0.46$ & $4.30 \pm 0.06$ & $11.08 \pm 0.06$ & $0.90 \pm 0.13$ & 2002 Mar 02 & $\mathrm{P}$ & yes(a) & \\
\hline HD 92855 & 1 & $2.934 \pm 0.005$ & $291.33 \pm 0.13$ & $4.57 \pm 0.09$ & $10.46 \pm 0.09$ & $0.75 \pm 0.15$ & 2002 Feb 01 & $\mathrm{P}$ & yes(a) & FM00 \\
\hline & 2 & $12.216 \pm 0.022$ & $147.79 \pm 0.25$ & $8.90 \pm 0.20$ & $14.79 \pm 0.20$ & $\ldots$ & 2002 Feb 01 & $\mathrm{P}$ & no(a) & \\
\hline GQ Leo & 1 & $0.248 \pm 0.002$ & $273.22 \pm 0.11$ & $0.13 \pm 0.06$ & $8.58 \pm 0.06$ & $1.02 \pm 0.08$ & 2003 Jan 12 & $\mathrm{P}$ & yes(a) & \\
\hline
\end{tabular}


Table 8

(Continued)

\begin{tabular}{|c|c|c|c|c|c|c|c|c|c|c|}
\hline Primary Star & $N_{C}$ & $\begin{array}{c}\rho \\
(\operatorname{arcsec})\end{array}$ & $\begin{array}{c}\theta \\
(\mathrm{deg})\end{array}$ & $\begin{array}{c}\Delta K_{S} \\
(\mathrm{mag})\end{array}$ & $\begin{array}{c}K_{S} \\
(\mathrm{mag})\end{array}$ & $\begin{array}{c}J-K_{S} \\
(\mathrm{mag})\end{array}$ & $\begin{array}{c}t_{0} \\
\text { (UT Date) }\end{array}$ & Tel. & Assoc. & Ref. \\
\hline & 2 & $10.038 \pm 0.009$ & $325.65 \pm 0.09$ & $6.40 \pm 0.06$ & $14.16 \pm 0.06$ & $0.13 \pm 0.09$ & 2003 Jan 12 & $\mathrm{P}$ & no(a) & \\
\hline HD 104576 & 1 & $10.455 \pm 0.028$ & $19.66 \pm 0.21$ & $11.00 \pm 0.50$ & $17.68 \pm 0.50$ & $\ldots$ & 2002 Jun 22 & $\mathrm{P}$ & no(e) & \\
\hline \multirow[t]{2}{*}{ HD 104860} & 1 & $3.803 \pm 0.027$ & $287.01 \pm 0.28$ & $10.92 \pm 0.25$ & $17.42 \pm 0.25$ & $0.00 \pm 0.47$ & 2002 Jun 23 & $\mathrm{P}$ & no(a) & \\
\hline & 2 & $11.961 \pm 0.033$ & $260.09 \pm 0.19$ & $12.09 \pm 0.18$ & $18.59 \pm 0.18$ & $\ldots$ & 2004 Jun 05 & $\mathrm{~K}$ & no(e) & \\
\hline SAO 15880 & 1 & $2.176 \pm 0.018$ & $293.93 \pm 0.72$ & $8.98 \pm 0.17$ & $16.27 \pm 0.17$ & $\ldots$ & $2004 \mathrm{Feb} 06$ & $\mathrm{P}$ & no(a) & \\
\hline HD 111456 & 1 & $3.783 \pm 0.010$ & $117.45 \pm 0.30$ & $12.72 \pm 0.16$ & $17.27 \pm 0.16$ & $\ldots$ & 2004 Feb 06 & $\mathrm{P}$ & $?$ & \\
\hline \multirow[t]{3}{*}{ HD 139498} & 1 & $0.311 \pm 0.002$ & $3.39 \pm 0.21$ & $0.00 \pm 0.02$ & $8.26 \pm 0.03$ & $0.50 \pm 0.05$ & $2003 \mathrm{Jul} 15$ & $\mathrm{P}$ & yes(a) & WDS \\
\hline & 2 & $11.246 \pm 0.033$ & $123.98 \pm 0.19$ & $8.48 \pm 0.30$ & $15.98 \pm 0.30$ & $\ldots$ & 2004 Jun 26 & $\mathrm{P}$ & $?$ & \\
\hline & 3 & $8.801 \pm 0.026$ & $61.50 \pm 0.21$ & $10.98 \pm 0.30$ & $18.49 \pm 0.30$ & $\ldots$ & 2004 Jun 26 & $P$ & $?$ & \\
\hline \multirow[t]{2}{*}{ HD 142361} & 1 & $0.705 \pm 0.001$ & $236.41 \pm 0.13$ & $2.01 \pm 0.10$ & $9.19 \pm 0.10$ & $0.85 \pm 0.14$ & 2002 Jun 21 & $\mathrm{P}$ & yes(a) & G93 \\
\hline & 2 & $11.207 \pm 0.046$ & $164.99 \pm 0.17$ & $5.85 \pm 0.17$ & $12.88 \pm 0.17$ & $0.77 \pm 0.28$ & 2002 Jun 21 & $\mathrm{P}$ & no(a) & \\
\hline \multirow[t]{5}{*}{ HD 143006} & 1 & $8.355 \pm 0.026$ & $130.27 \pm 0.25$ & $9.28 \pm 0.16$ & $16.33 \pm 0.16$ & $1.18 \pm 0.23$ & 2002 Jun 23 & $\mathrm{P}$ & $?$ & \\
\hline & 2 & $6.626 \pm 0.028$ & $0.32 \pm 0.23$ & $10.40 \pm 0.16$ & $17.45 \pm 0.16$ & $1.40 \pm 0.23$ & 2002 Jun 23 & $\mathrm{P}$ & $?$ & \\
\hline & 3 & $8.502 \pm 0.029$ & $268.41 \pm 0.23$ & $10.66 \pm 0.16$ & $17.71 \pm 0.16$ & $1.27 \pm 0.23$ & 2002 Jun 23 & $\mathrm{P}$ & no(a) & \\
\hline & 4 & $7.698 \pm 0.023$ & $357.97 \pm 0.12$ & $12.11 \pm 0.16$ & $19.16 \pm 0.16$ & $1.30 \pm 0.23$ & 2003 May 18 & $\mathrm{~K}$ & $?$ & \\
\hline & 5 & $12.279 \pm 0.028$ & $102.74 \pm 0.12$ & $11.29 \pm 0.16$ & $18.34 \pm 0.16$ & $0.88 \pm 0.23$ & 2003 May 18 & $\mathrm{~K}$ & $?$ & \\
\hline PZ99 J155847.8-175800 & 1 & $9.118 \pm 0.034$ & $224.72 \pm 0.35$ & $11.25 \pm 0.22$ & $19.58 \pm 0.22$ & $\ldots$ & 2004 Jun 06 & $\mathrm{~K}$ & $?$ & \\
\hline \multirow{3}{*}{ ScoPMS 21} & 1 & $6.221 \pm 0.014$ & $36.94 \pm 0.13$ & $7.39 \pm 0.02$ & $15.91 \pm 0.03$ & $1.05 \pm 0.05$ & 2002 Jun 22 & $\mathrm{P}$ & no(a) & \\
\hline & 2 & $9.888 \pm 0.027$ & $74.26 \pm 0.18$ & $8.06 \pm 0.04$ & $16.58 \pm 0.05$ & $0.67 \pm 0.06$ & 2002 Jun 22 & $\mathrm{P}$ & $\operatorname{no}(\mathrm{c})$ & \\
\hline & 3 & $9.351 \pm 0.020$ & $308.13 \pm 0.16$ & $8.93 \pm 0.02$ & $17.45 \pm 0.03$ & $1.11 \pm 0.08$ & 2002 Jun 22 & $\mathrm{P}$ & no(a) & \\
\hline \multirow[t]{4}{*}{ PZ99 J160302.7-180605 } & 1 & $1.572 \pm 0.006$ & $190.97 \pm 0.19$ & $9.59 \pm 0.09$ & $18.32 \pm 0.09$ & $2.42 \pm 0.17$ & 2003 May 18 & $\mathrm{~K}$ & no(a) & \\
\hline & 2 & $5.797 \pm 0.013$ & $272.51 \pm 0.13$ & $7.58 \pm 0.62$ & $16.31 \pm 0.62$ & $\ldots$ & 2003 May 18 & $\mathrm{~K}$ & no(a) & \\
\hline & 3 & $9.065 \pm 0.020$ & $73.35 \pm 0.12$ & $10.58 \pm 0.49$ & $19.31 \pm 0.49$ & $\ldots$ & 2003 May 18 & $\mathrm{~K}$ & $?$ & \\
\hline & 4 & $9.653 \pm 0.023$ & $107.18 \pm 0.12$ & $11.81 \pm 0.53$ & $20.54 \pm 0.53$ & $\ldots$ & 2003 May 18 & $\mathrm{~K}$ & $?$ & \\
\hline \multirow[t]{4}{*}{ ScoPMS 27} & 1 & $0.079 \pm 0.006$ & $77.04 \pm 0.77$ & $0.60 \pm 0.20$ & $9.14 \pm 0.20$ & $0.83 \pm 0.28$ & 2004 Jun 28 & $\mathrm{P}$ & yes $(c)$ & \\
\hline & 2 & $11.113 \pm 0.025$ & $218.10 \pm 0.22$ & $10.33 \pm 0.30$ & $18.37 \pm 0.30$ & $\ldots$ & 2004 Jun 28 & $P$ & no(a) & \\
\hline & 3 & $5.807 \pm 0.027$ & $87.35 \pm 0.20$ & $10.59 \pm 0.30$ & $18.63 \pm 0.30$ & $\ldots$ & 2004 Jun 28 & $\mathrm{P}$ & $?$ & \\
\hline & 4 & $5.346 \pm 0.026$ & $336.58 \pm 0.21$ & $10.47 \pm 0.30$ & $18.51 \pm 0.30$ & $\ldots$ & 2004 Jun 28 & $P$ & $?$ & \\
\hline ScoPMS 52 & 1 & $0.144 \pm 0.005$ & $162.15 \pm 1.76$ & $1.10 \pm 0.10$ & $8.93 \pm 0.10$ & $\ldots$ & 2002 Aug 31 & $\mathrm{P}$ & yes(a) & G93 \\
\hline \multirow[t]{5}{*}{ PZ99 J161318.6-221248 } & 1 & $3.770 \pm 0.012$ & $313.46 \pm 0.22$ & $11.00 \pm 0.10$ & $18.43 \pm 0.10$ & $\ldots$ & 2002 Jun 21 & $\mathrm{P}$ & no(a) & \\
\hline & 2 & $3.333 \pm 0.021$ & $81.19 \pm 0.41$ & $11.20 \pm 0.10$ & $18.63 \pm 0.10$ & $\ldots$ & 2002 Jun 21 & $\mathrm{P}$ & no(a) & \\
\hline & 3 & $8.860 \pm 0.034$ & $77.36 \pm 0.23$ & $11.00 \pm 0.20$ & $18.43 \pm 0.20$ & $\ldots$ & 2002 Jun 21 & $\mathrm{P}$ & no(a) & \\
\hline & 4 & $7.957 \pm 0.018$ & $152.07 \pm 0.13$ & $10.83 \pm 0.22$ & $18.26 \pm 0.22$ & $\ldots$ & 2003 May 18 & $\mathrm{~K}$ & $?$ & \\
\hline & 5 & $12.182 \pm 0.029$ & $259.12 \pm 0.17$ & $10.72 \pm 0.21$ & $18.15 \pm 0.21$ & $\ldots$ & 2003 May 18 & $\mathrm{~K}$ & $?$ & \\
\hline \multirow[t]{3}{*}{ PZ99 J161402.1-230101 } & 1 & $5.366 \pm 0.030$ & $356.14 \pm 0.49$ & $7.76 \pm 0.12$ & $16.37 \pm 0.12$ & $\ldots$ & 2003 Jul 15 & $\mathrm{P}$ & $?$ & \\
\hline & 2 & $9.633 \pm 0.015$ & $128.34 \pm 0.15$ & $9.16 \pm 0.17$ & $17.77 \pm 0.17$ & $\ldots$ & 2004 Jun 05 & K & $?$ & \\
\hline & 3 & $7.858 \pm 0.017$ & $281.13 \pm 0.13$ & $10.35 \pm 0.17$ & $18.96 \pm 0.17$ & $\ldots$ & 2004 Jun 05 & $\mathrm{~K}$ & $?$ & \\
\hline \multirow[t]{7}{*}{ PZ99 J161411.0-230536 } & 1 & $0.222 \pm 0.003$ & $304.76 \pm 0.41$ & $0.21 \pm 0.10$ & $8.32 \pm 0.10$ & $0.84 \pm 0.18$ & 2002 Jun 21 & $\mathrm{P}$ & yes(a) & \\
\hline & 2 & $2.659 \pm 0.007$ & $100.46 \pm 0.21$ & $6.26 \pm 0.03$ & $13.72 \pm 0.04$ & $0.82 \pm 0.11$ & 2002 Jun 21 & $\mathrm{P}$ & no(a) & \\
\hline & 3 & $2.808 \pm 0.010$ & $98.36 \pm 0.14$ & $10.25 \pm 0.50$ & $18.73 \pm 0.50$ & $\ldots$ & 2004 Jun 05 & $\mathrm{~K}$ & $?$ & \\
\hline & 4 & $7.709 \pm 0.017$ & $341.92 \pm 0.12$ & $8.16 \pm 0.10$ & $15.62 \pm 0.10$ & $0.33 \pm 0.22$ & 2004 Jun 05 & $\mathrm{~K}$ & $\operatorname{no}(\mathrm{c})$ & \\
\hline & 5 & $8.037 \pm 0.018$ & $145.10 \pm 0.12$ & $9.50 \pm 0.06$ & $16.96 \pm 0.07$ & $\ldots$ & 2004 Jun 05 & $\mathrm{~K}$ & $?$ & \\
\hline & 6 & $8.926 \pm 0.021$ & $80.29 \pm 0.12$ & $11.72 \pm 0.17$ & $19.18 \pm 0.17$ & $\ldots$ & 2004 Jun 05 & $\mathrm{~K}$ & $?$ & \\
\hline & 7 & $9.243 \pm 0.021$ & $69.00 \pm 0.15$ & $12.51 \pm 0.20$ & $19.97 \pm 0.20$ & $\ldots$ & 2004 Jun 05 & $\mathrm{~K}$ & $?$ & \\
\hline \multirow[t]{7}{*}{ PZ99 J161459.2-275023 } & 1 & $4.787 \pm 0.025$ & $264.80 \pm 0.20$ & $7.07 \pm 0.15$ & $15.76 \pm 0.15$ & $\ldots$ & $2003 \mathrm{Jul} 15$ & $\mathrm{P}$ & $?$ & \\
\hline & 2 & $5.554 \pm 0.072$ & $187.48 \pm 0.58$ & $7.40 \pm 0.15$ & $16.09 \pm 0.15$ & $\ldots$ & 2003 Jul 15 & $P$ & $?$ & \\
\hline & 3 & $3.919 \pm 0.010$ & $153.63 \pm 0.13$ & $9.65 \pm 0.17$ & $18.34 \pm 0.17$ & $\cdots$ & 2004 Jun 05 & $\mathrm{~K}$ & $?$ & \\
\hline & 4 & $12.299 \pm 0.027$ & $253.55 \pm 0.12$ & $8.89 \pm 0.18$ & $17.58 \pm 0.18$ & $\ldots$ & 2004 Jun 05 & $\mathrm{~K}$ & $?$ & \\
\hline & 5 & $11.464 \pm 0.026$ & $72.94 \pm 0.12$ & $9.91 \pm 0.17$ & $18.60 \pm 0.17$ & $\ldots$ & 2004 Jun 05 & $\mathrm{~K}$ & $?$ & \\
\hline & 6 & $6.241 \pm 0.015$ & $259.72 \pm 0.17$ & $10.65 \pm 0.18$ & $19.34 \pm 0.18$ & $\ldots$ & 2004 Jun 05 & $\mathrm{~K}$ & $?$ & \\
\hline & 7 & $7.936 \pm 0.018$ & $55.55 \pm 0.14$ & $10.59 \pm 0.19$ & $19.28 \pm 0.19$ & $\ldots$ & 2004 Jun 05 & $\mathrm{~K}$ & $?$ & \\
\hline PZ99 J161618.0-233947 & 1 & $9.119 \pm 0.028$ & $160.44 \pm 0.06$ & $7.26 \pm 0.30$ & $15.36 \pm 0.30$ & $\cdots$ & 2004 Jun 27 & $\mathrm{P}$ & $?$ & \\
\hline & 2 & $10.049 \pm 0.026$ & $195.43 \pm 0.06$ & $7.57 \pm 0.13$ & $15.67 \pm 0.13$ & $\cdots$ & 2004 Jun 27 & $\mathrm{P}$ & $?$ & \\
\hline & 3 & $3.190 \pm 0.015$ & $184.47 \pm 0.64$ & $10.44 \pm 0.15$ & $18.54 \pm 0.15$ & $\cdots$ & 2004 Jun 27 & $\mathrm{P}$ & $?$ & \\
\hline & 4 & $10.938 \pm 0.021$ & $165.25 \pm 0.18$ & $10.03 \pm 0.11$ & $18.13 \pm 0.11$ & $\ldots$ & 2004 Jun 27 & $\mathrm{P}$ & $?$ & \\
\hline & 5 & $12.451 \pm 0.020$ & $251.52 \pm 0.20$ & $9.31 \pm 0.18$ & $17.41 \pm 0.18$ & $\ldots$ & 2004 Jun 27 & $\mathrm{P}$ & $?$ & \\
\hline & 6 & $3.671 \pm 0.025$ & $140.63 \pm 0.22$ & $10.81 \pm 0.30$ & $18.91 \pm 0.30$ & $\ldots$ & 2004 Jun 27 & $P$ & $?$ & \\
\hline & 7 & $6.845 \pm 0.025$ & $144.15 \pm 0.22$ & $10.70 \pm 0.30$ & $18.80 \pm 0.30$ & $\ldots$ & 2004 Jun 27 & $\mathrm{P}$ & $?$ & \\
\hline & 8 & $9.452 \pm 0.028$ & $108.96 \pm 0.21$ & $10.68 \pm 0.30$ & $18.78 \pm 0.30$ & $\ldots$ & 2004 Jun 27 & $\mathrm{P}$ & $?$ & \\
\hline & 9 & $12.309 \pm 0.025$ & $38.57 \pm 0.22$ & $10.51 \pm 0.30$ & $18.61 \pm 0.30$ & $\ldots$ & 2004 Jun 27 & $\mathrm{P}$ & $?$ & \\
\hline & 10 & $3.711 \pm 0.026$ & $184.89 \pm 0.20$ & $11.43 \pm 0.30$ & $19.53 \pm 0.30$ & $\ldots$ & 2004 Jun 27 & $P$ & $?$ & \\
\hline HD 146516 & 1 & $5.738 \pm 0.012$ & $222.79 \pm 0.13$ & $7.60 \pm 0.09$ & $15.57 \pm 0.09$ & $0.80 \pm 0.15$ & 2003 May 10 & $P$ & no(a) & \\
\hline & 2 & $9.218 \pm 0.028$ & $333.77 \pm 0.14$ & $7.50 \pm 0.09$ & $15.47 \pm 0.09$ & $0.74 \pm 0.15$ & 2003 May 10 & $\mathrm{P}$ & ? & \\
\hline & 3 & $9.493 \pm 0.021$ & $81.79 \pm 0.12$ & $10.40 \pm 0.18$ & $18.37 \pm 0.18$ & $\ldots$ & 2004 Jun 05 & $\mathrm{~K}$ & $?$ & \\
\hline
\end{tabular}


Table 8

(Continued)

\begin{tabular}{|c|c|c|c|c|c|c|c|c|c|c|}
\hline Primary Star & $N_{C}$ & $\begin{array}{c}\rho \\
(\operatorname{arcsec})\end{array}$ & $\begin{array}{c}\theta \\
(\operatorname{deg})\end{array}$ & $\begin{array}{c}\Delta K_{S} \\
(\mathrm{mag})\end{array}$ & $\begin{array}{c}K_{S} \\
(\mathrm{mag})\end{array}$ & $\begin{array}{c}J-K_{S} \\
(\mathrm{mag})\end{array}$ & $\begin{array}{c}t_{0} \\
\text { (UT Date) }\end{array}$ & Tel. & Assoc. & Ref. \\
\hline & 4 & $6.256 \pm 0.020$ & $350.00 \pm 0.13$ & $11.67 \pm 0.23$ & $19.64 \pm 0.23$ & $\ldots$ & 2004 Jun 05 & $\mathrm{~K}$ & $?$ & \\
\hline \multirow[t]{7}{*}{ ScoPMS 214} & 1 & $3.070 \pm 0.010$ & $121.17 \pm 0.23$ & $5.96 \pm 0.09$ & $13.72 \pm 0.09$ & $1.06 \pm 0.13$ & 2002 Aug 30 & $\mathrm{P}$ & no $^{\mathrm{d}}$ & \\
\hline & 2 & $3.598 \pm 0.009$ & $350.09 \pm 0.24$ & $8.95 \pm 0.02$ & $16.71 \pm 0.03$ & $1.18 \pm 0.05$ & 2002 Aug 30 & $\mathrm{P}$ & no(a) & \\
\hline & 3 & $4.623 \pm 0.013$ & $349.37 \pm 0.19$ & $9.87 \pm 0.04$ & $17.63 \pm 0.05$ & $1.22 \pm 0.09$ & 2002 Aug 30 & $\mathrm{P}$ & no(a) & \\
\hline & 4 & $10.371 \pm 0.019$ & $353.28 \pm 0.14$ & $8.64 \pm 0.08$ & $16.40 \pm 0.08$ & $0.70 \pm 0.09$ & 2002 Aug 30 & $\mathrm{P}$ & no(a) & \\
\hline & 5 & $9.674 \pm 0.030$ & $180.59 \pm 0.23$ & $10.72 \pm 0.30$ & $18.48 \pm 0.30$ & $\ldots$ & 2002 Aug 30 & $\mathrm{P}$ & $?$ & \\
\hline & 6 & $10.229 \pm 0.034$ & $137.12 \pm 0.26$ & $11.70 \pm 0.30$ & $19.46 \pm 0.30$ & $\ldots$ & 2002 Aug 30 & $\mathrm{P}$ & $?$ & \\
\hline & 7 & $10.202 \pm 0.032$ & $351.32 \pm 0.13$ & $11.14 \pm 0.18$ & $18.90 \pm 0.18$ & $\ldots$ & 2004 Jun 05 & $\mathrm{~K}$ & no(a) & \\
\hline \multirow[t]{9}{*}{ HD 151798} & 1 & $10.330 \pm 0.018$ & $335.94 \pm 0.17$ & $7.76 \pm 0.02$ & $14.24 \pm 0.02$ & $0.90 \pm 0.04$ & 2002 Jun 21 & $\mathrm{P}$ & no(a) & \\
\hline & 2 & $4.682 \pm 0.013$ & $15.84 \pm 0.22$ & $10.40 \pm 0.30$ & $16.88 \pm 0.30$ & $\ldots$ & 2002 Jun 21 & $\mathrm{P}$ & no(a) & \\
\hline & 3 & $7.363 \pm 0.014$ & $11.87 \pm 0.17$ & $11.66 \pm 0.10$ & $18.14 \pm 0.10$ & $\ldots$ & 2002 Jun 21 & $\mathrm{P}$ & no(a) & \\
\hline & 4 & $4.212 \pm 0.042$ & $222.87 \pm 0.29$ & $14.07 \pm 0.17$ & $20.55 \pm 0.17$ & $\ldots$ & 2003 May 16 & $\mathrm{~K}$ & $?$ & \\
\hline & 5 & $7.393 \pm 0.087$ & $198.06 \pm 0.27$ & $13.87 \pm 0.30$ & $20.35 \pm 0.30$ & $\ldots$ & 2003 May 16 & K & $?$ & \\
\hline & 6 & $8.391 \pm 0.062$ & $132.59 \pm 0.32$ & $12.66 \pm 0.38$ & $19.14 \pm 0.38$ & $\ldots$ & 2003 May 16 & $\mathrm{~K}$ & $?$ & \\
\hline & 7 & $6.737 \pm 0.041$ & $117.42 \pm 0.50$ & $12.92 \pm 0.36$ & $19.40 \pm 0.36$ & $\ldots$ & 2003 May 16 & $\mathrm{~K}$ & $?$ & \\
\hline & 8 & $8.609 \pm 0.077$ & $188.86 \pm 0.22$ & $13.15 \pm 0.54$ & $19.63 \pm 0.54$ & $\ldots$ & 2003 May 16 & K & $?$ & \\
\hline & 9 & $6.635 \pm 0.046$ & $255.47 \pm 0.29$ & $13.17 \pm 0.17$ & $19.65 \pm 0.17$ & $\ldots$ & 2003 May 16 & $\mathrm{~K}$ & $?$ & \\
\hline \multirow[t]{4}{*}{ HD 165590} & 1 & $0.446 \pm 0.001$ & $90.22 \pm 0.17$ & $0.68 \pm 0.01$ & $6.50 \pm 0.02$ & $\ldots$ & 2004 Jun 28 & $\mathrm{P}$ & yes(p) & Hip \\
\hline & 2 & $2.599 \pm 0.015$ & $62.65 \pm 1.16$ & $8.52 \pm 0.10$ & $14.34 \pm 0.10$ & $\ldots$ & 2004 Jun 28 & $\mathrm{P}$ & no(a) & \\
\hline & 3 & $12.462 \pm 0.033$ & $33.35 \pm 0.24$ & $10.56 \pm 0.10$ & $16.38 \pm 0.10$ & $\ldots$ & 2004 Jun 28 & $\mathrm{P}$ & no(a) & \\
\hline & 4 & $6.548 \pm 0.011$ & $111.35 \pm 0.26$ & $12.97 \pm 0.10$ & $18.79 \pm 0.10$ & $\ldots$ & 2004 Jun 28 & $\mathrm{P}$ & no(a) & \\
\hline HD 170778 & 1 & $10.103 \pm 0.057$ & $39.62 \pm 0.39$ & $12.09 \pm 0.11$ & $18.14 \pm 0.11$ & $\ldots$ & 2004 Jun 27 & $\mathrm{P}$ & no(a) & \\
\hline \multirow[t]{5}{*}{ HD 171488} & 1 & $2.620 \pm 0.006$ & $30.85 \pm 0.12$ & $6.72 \pm 0.24$ & $12.57 \pm 0.24$ & $0.59 \pm 0.34$ & 2004 Jun 06 & $\mathrm{~K}$ & no(a) & \\
\hline & 2 & $1.796 \pm 0.008$ & $86.65 \pm 0.22$ & $11.02 \pm 0.24$ & $16.87 \pm 0.24$ & $0.39 \pm 0.34$ & 2004 Jun 06 & K & no(a) & \\
\hline & 3 & $6.178 \pm 0.015$ & $306.56 \pm 0.13$ & $12.04 \pm 0.24$ & $17.89 \pm 0.24$ & $1.15 \pm 0.34$ & 2004 Jun 06 & $\mathrm{~K}$ & $\operatorname{no}(\mathrm{c})$ & \\
\hline & 4 & $12.301 \pm 0.026$ & $181.69 \pm 0.12$ & $11.69 \pm 0.10$ & $17.54 \pm 0.10$ & $\ldots$ & 2004 Jun 06 & K & $?$ & \\
\hline & 5 & $6.870 \pm 0.017$ & $114.19 \pm 0.14$ & $12.30 \pm 0.10$ & $18.15 \pm 0.10$ & $\ldots$ & 2004 Jun 06 & $\mathrm{~K}$ & $?$ & \\
\hline \multirow[t]{6}{*}{ HD 172649} & 1 & $4.829 \pm 0.011$ & $356.29 \pm 0.25$ & $6.62 \pm 0.07$ & $12.85 \pm 0.07$ & $0.47 \pm 0.26$ & 2002 Jun 21 & $\mathrm{P}$ & no(a) & \\
\hline & 2 & $2.092 \pm 0.005$ & $344.27 \pm 0.49$ & $8.80 \pm 0.07$ & $15.03 \pm 0.07$ & $0.61 \pm 0.08$ & 2002 Jun 21 & $\mathrm{P}$ & no(a) & \\
\hline & 3 & $8.570 \pm 0.026$ & $33.81 \pm 0.12$ & $9.85 \pm 0.08$ & $16.08 \pm 0.08$ & $0.80 \pm 0.09$ & 2002 Aug 31 & $\mathrm{P}$ & no(a) & \\
\hline & 4 & $11.795 \pm 0.025$ & $110.75 \pm 0.17$ & $11.64 \pm 0.11$ & $17.87 \pm 0.11$ & $0.98 \pm 0.13$ & 2002 Aug 31 & $\mathrm{P}$ & no(a) & \\
\hline & 5 & $11.771 \pm 0.021$ & $109.54 \pm 0.19$ & $13.01 \pm 0.40$ & $19.24 \pm 0.40$ & $\ldots$ & 2002 Aug 31 & $\mathrm{P}$ & no(a) & \\
\hline & 6 & $7.847 \pm 0.030$ & $354.63 \pm 0.26$ & $13.77 \pm 0.30$ & $20.00 \pm 0.30$ & $\ldots$ & 2002 Aug 31 & $\mathrm{P}$ & $?$ & \\
\hline \multirow[t]{2}{*}{ HD 187748} & 1 & $7.924 \pm 0.053$ & $276.61 \pm 0.20$ & $12.00 \pm 0.04$ & $17.26 \pm 0.05$ & $\ldots$ & 2004 Jun 27 & $\mathrm{P}$ & no(a) & \\
\hline & 2 & $7.848 \pm 0.044$ & $277.01 \pm 0.25$ & $12.30 \pm 0.12$ & $17.56 \pm 0.12$ & $\ldots$ & 2004 Jun 27 & $\mathrm{P}$ & no(a) & \\
\hline \multirow[t]{2}{*}{ HD 191089} & 1 & $10.893 \pm 0.022$ & $219.80 \pm 0.13$ & $7.80 \pm 0.20$ & $13.88 \pm 0.20$ & $\ldots$ & 2003 Sep 20 & $\mathrm{P}$ & no(a) & \\
\hline & 2 & $10.727 \pm 0.030$ & $147.93 \pm 0.23$ & $12.36 \pm 0.20$ & $18.44 \pm 0.20$ & $\ldots$ & 2003 Sep 20 & $\mathrm{P}$ & $?$ & \\
\hline \multirow[t]{3}{*}{ HD 200746} & 1 & $0.227 \pm 0.049$ & $355.26 \pm 1.12$ & $1.70 \pm 0.20$ & $8.29 \pm 0.20$ & $0.75 \pm 0.36$ & 2003 Sep 21 & $\mathrm{P}$ & yes(a) & Hip \\
\hline & 2 & $4.295 \pm 0.026$ & $353.99 \pm 0.23$ & $11.20 \pm 0.15$ & $17.59 \pm 0.15$ & $\ldots$ & 2003 Sep 21 & $\mathrm{P}$ & no(a) & \\
\hline & 3 & $9.806 \pm 0.029$ & $165.81 \pm 0.23$ & $11.70 \pm 0.16$ & $18.09 \pm 0.16$ & $\ldots$ & 2003 Sep 21 & $\mathrm{P}$ & $?$ & \\
\hline \multirow[t]{6}{*}{ HD 203030} & 1 & $8.579 \pm 0.014$ & $314.20 \pm 0.12$ & $6.21 \pm 0.09$ & $12.86 \pm 0.09$ & $0.62 \pm 0.15$ & 2002 Aug 28 & $\mathrm{P}$ & no(a) & \\
\hline & 2 & $8.610 \pm 0.015$ & $318.36 \pm 0.12$ & $8.42 \pm 0.09$ & $15.07 \pm 0.09$ & $0.84 \pm 0.17$ & 2002 Aug 28 & $\mathrm{P}$ & no(a) & \\
\hline & 3 & $11.923 \pm 0.021$ & $108.76 \pm 0.12$ & $9.58 \pm 0.11$ & $16.23 \pm 0.11$ & $1.92 \pm 0.56$ & 2002 Aug 28 & $\mathrm{P}$ & yes(a) & MH06 \\
\hline & 4 & $12.137 \pm 0.019$ & $215.15 \pm 0.12$ & $8.69 \pm 0.11$ & $15.34 \pm 0.11$ & $0.53 \pm 0.17$ & 2002 Aug 28 & $\mathrm{P}$ & no(a) & \\
\hline & 5 & $9.933 \pm 0.027$ & $218.43 \pm 0.20$ & $11.29 \pm 0.08$ & $17.94 \pm 0.08$ & $\ldots$ & 2002 Aug 28 & $\mathrm{P}$ & no(a) & \\
\hline & 6 & $3.365 \pm 0.025$ & $343.13 \pm 0.23$ & $11.76 \pm 0.30$ & $18.41 \pm 0.30$ & $\ldots$ & 2002 Aug 28 & $\mathrm{P}$ & no(a) & \\
\hline \multirow[t]{4}{*}{ HD 209393} & 1 & $9.187 \pm 0.018$ & $6.57 \pm 0.13$ & $10.81 \pm 0.10$ & $17.13 \pm 0.10$ & $\ldots$ & 2002 Nov 17 & $\mathrm{P}$ & no(a) & \\
\hline & 2 & $8.188 \pm 0.017$ & $71.50 \pm 0.14$ & $12.80 \pm 0.20$ & $19.12 \pm 0.20$ & $\ldots$ & 2002 Nov 17 & $\mathrm{P}$ & no(a) & \\
\hline & 3 & $6.237 \pm 0.013$ & $317.66 \pm 0.18$ & $13.11 \pm 0.22$ & $19.43 \pm 0.22$ & $\ldots$ & 2002 Nov 17 & $\mathrm{P}$ & no(a) & \\
\hline & 4 & $10.344 \pm 0.025$ & $210.77 \pm 0.15$ & $12.83 \pm 0.11$ & $19.15 \pm 0.11$ & $\ldots$ & 2002 Nov 17 & $\mathrm{P}$ & no(a) & \\
\hline \multirow[t]{6}{*}{ V383 Lac } & 1 & $10.736 \pm 0.020$ & $91.89 \pm 0.16$ & $8.74 \pm 0.04$ & $15.24 \pm 0.05$ & $\ldots$ & 2002 Aug 27 & $\mathrm{P}$ & no(a) & \\
\hline & 2 & $11.744 \pm 0.024$ & $140.12 \pm 0.14$ & $11.12 \pm 0.09$ & $17.62 \pm 0.09$ & $\ldots$ & 2002 Aug 27 & $\mathrm{P}$ & no(a) & \\
\hline & 3 & $9.240 \pm 0.017$ & $108.35 \pm 0.18$ & $10.97 \pm 0.08$ & $17.47 \pm 0.08$ & $\ldots$ & 2002 Aug 27 & $\mathrm{P}$ & no(a) & \\
\hline & 4 & $4.427 \pm 0.018$ & $200.35 \pm 0.16$ & $11.10 \pm 0.12$ & $17.61 \pm 0.12$ & $\ldots$ & 2002 Aug 27 & $\mathrm{P}$ & no(a) & \\
\hline & 5 & $4.231 \pm 0.010$ & $98.09 \pm 0.56$ & $11.57 \pm 0.11$ & $18.08 \pm 0.11$ & $\ldots$ & 2002 Aug 27 & $\mathrm{P}$ & no(a) & \\
\hline & 6 & $11.594 \pm 0.025$ & $270.42 \pm 0.13$ & $11.44 \pm 0.10$ & $17.94 \pm 0.10$ & $\ldots$ & 2002 Aug 27 & $\mathrm{P}$ & no(a) & \\
\hline HD 218738 & 1 & $10.619 \pm 0.026$ & $97.59 \pm 0.17$ & $8.10 \pm 1.00$ & $13.76 \pm 1.00$ & $\ldots$ & 2003 Dec 10 & $\mathrm{P}$ & no(a) & \\
\hline & 2 & $10.616 \pm 0.025$ & $97.81 \pm 0.18$ & $8.07 \pm 1.00$ & $13.73 \pm 1.00$ & $\ldots$ & 2003 Dec 10 & $\mathrm{P}$ & no(a) & \\
\hline & 3 & $4.144 \pm 0.014$ & $182.07 \pm 0.23$ & $10.09 \pm 0.23$ & $15.75 \pm 0.23$ & $\ldots$ & 2003 Dec 10 & $\mathrm{P}$ & no(a) & \\
\hline & 4 & $5.339 \pm 0.016$ & $120.60 \pm 0.17$ & $11.83 \pm 0.27$ & $17.49 \pm 0.27$ & $\ldots$ & 2003 Dec 10 & $\mathrm{P}$ & no(a) & \\
\hline & 5 & $6.022 \pm 0.022$ & $38.28 \pm 0.25$ & $12.68 \pm 0.25$ & $18.34 \pm 0.25$ & $\ldots$ & 2003 Dec 10 & $\mathrm{P}$ & no(a) & \\
\hline & 6 & $5.508 \pm 0.028$ & $33.36 \pm 0.26$ & $13.70 \pm 0.50$ & $19.36 \pm 0.50$ & $\ldots$ & 2003 Dec 10 & $\mathrm{P}$ & no(a) & \\
\hline & 7 & $9.186 \pm 0.032$ & $42.22 \pm 0.26$ & $13.58 \pm 0.30$ & $19.24 \pm 0.30$ & $\ldots$ & 2003 Dec 10 & $\mathrm{P}$ & $?$ & \\
\hline & 8 & $2.134 \pm 0.018$ & $224.02 \pm 0.33$ & $10.96 \pm 0.50$ & $16.62 \pm 0.50$ & $\ldots$ & 2004 Oct 04 & $\mathrm{P}$ & no(a) & \\
\hline
\end{tabular}


Table 8

(Continued)

\begin{tabular}{|c|c|c|c|c|c|c|c|c|c|c|}
\hline Primary Star & $N_{C}$ & $\begin{array}{c}\rho \\
(\operatorname{arcsec})\end{array}$ & $\begin{array}{c}\theta \\
(\operatorname{deg})\end{array}$ & $\begin{array}{c}\Delta K_{S} \\
(\mathrm{mag})\end{array}$ & $\begin{array}{c}K_{S} \\
(\mathrm{mag})\end{array}$ & $\begin{array}{c}J-K_{S} \\
(\mathrm{mag})\end{array}$ & $\begin{array}{c}t_{0} \\
\text { (UT Date) }\end{array}$ & Tel. & Assoc. & Ref. \\
\hline \multirow[t]{3}{*}{ HD 218739} & 1 & $7.050 \pm 0.030$ & $221.73 \pm 0.34$ & $7.45 \pm 0.50$ & $13.12 \pm 0.50$ & $\ldots$ & 2003 Dec 10 & $\mathrm{P}$ & no(a) & \\
\hline & 2 & $12.055 \pm 0.055$ & $238.54 \pm 0.34$ & $12.78 \pm 0.30$ & $18.45 \pm 0.30$ & $\ldots$ & 2003 Dec 10 & $\mathrm{P}$ & no(a) & \\
\hline & 3 & $9.526 \pm 0.039$ & $287.92 \pm 0.34$ & $13.16 \pm 0.50$ & $18.83 \pm 0.50$ & $\ldots$ & 2003 Dec 10 & $\mathrm{P}$ & no(a) & \\
\hline \multirow[t]{2}{*}{ HD 219498} & 1 & $8.862 \pm 0.022$ & $129.85 \pm 0.19$ & $8.69 \pm 0.14$ & $16.07 \pm 0.14$ & $\ldots$ & 2002 Aug 30 & $\mathrm{P}$ & no(a) & \\
\hline & 2 & $9.792 \pm 0.032$ & $305.61 \pm 0.25$ & $11.76 \pm 0.07$ & $19.14 \pm 0.07$ & $\ldots$ & 2002 Aug 30 & $\mathrm{P}$ & no(a) & \\
\hline
\end{tabular}

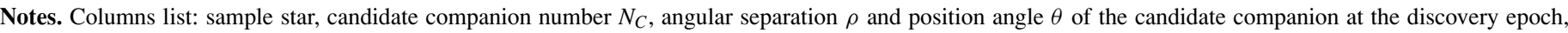

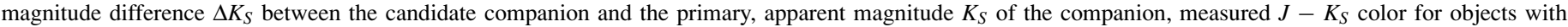

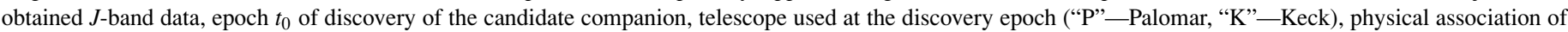

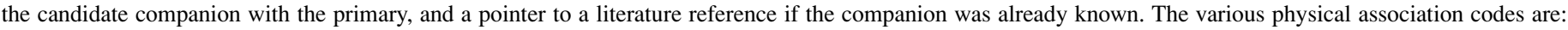

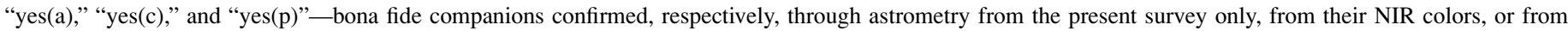

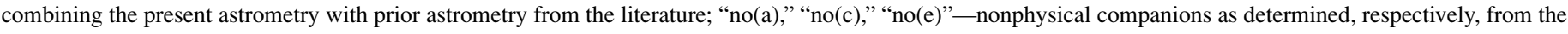
present astrometry, from their NIR colors, or based on a nonpoint-like extended PSF; "?" —undecided candidate companions.

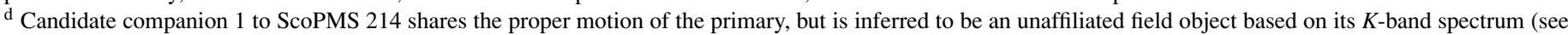
Section 6.3).

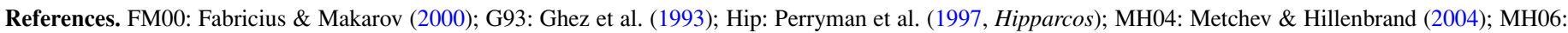
Metchev \& Hillenbrand (2006); WDS: Mason et al. (2001; Washington Double Star Catalog, and references therein).

(This table is also available in a machine-readable form in the online journal.)

source PSF (which suggests an extragalactic object). Candidate substellar companions that satisfied the common proper motion test were also spectroscopically observed to confirm that their spectral types were in agreement with their projected substellar masses.

\subsubsection{Proper Motion}

Proper motion is usually the criterion of choice in companion studies, as it provides nearly unambiguous evidence of association between two objects: whether as components of a gravitationally bound system or as members of a multistar moving group sharing a common origin. We used the common proper motion criterion through the combined application of two requirements: (1) that the change in the position of the candidate companions relative to the primaries was within $3 \sigma$ of zero in all of right ascension $(\alpha)$, declination $(\delta)$, angular separation $(\rho)$, and position angle $(\theta)$; and (2) that the expected change in relative positions of the candidate companions, had they been stationary background objects, was more than $3 \sigma$ discrepant in either $\alpha, \delta, \rho$, or $\theta$ from the observed change. Often, in cases of candidate close $(\lesssim 20 \mathrm{AU})$ binaries, criterion (1) was not satisfied because of appreciable orbital motion. In such situations, we instead made sure that (3) the observed change in relative position was much smaller (and less significant) than the expected change if the components of the candidate binary were not gravitationally bound. A detailed example of the implementation of the above astrometric criteria is worked out in Metchev (2006, Chapter 5.4.1).

When a relatively bright field star (4 mag $<\Delta K_{S} \lesssim$ $8 \mathrm{mag}$ ) was present in the deep coronagraphic exposures at Palomar, its position in the shallow noncoronagraphic images was used as an additional astrometric reference. In cases where the subsequent astrometric measurements with respect to the primary and to fainter field objects showed such bright field stars to be approximately stationary, they could be used to bootstrap the association of other candidate companions with the primary, and thus circumventing the somewhat higher positional uncertainty associated with locating the primary behind the opaque PHARO coronagraph. This technique was particularly important in determining the association of systems in the distant Upper Scorpius (145 pc) and $\alpha$ Persei (190 pc) regions, where

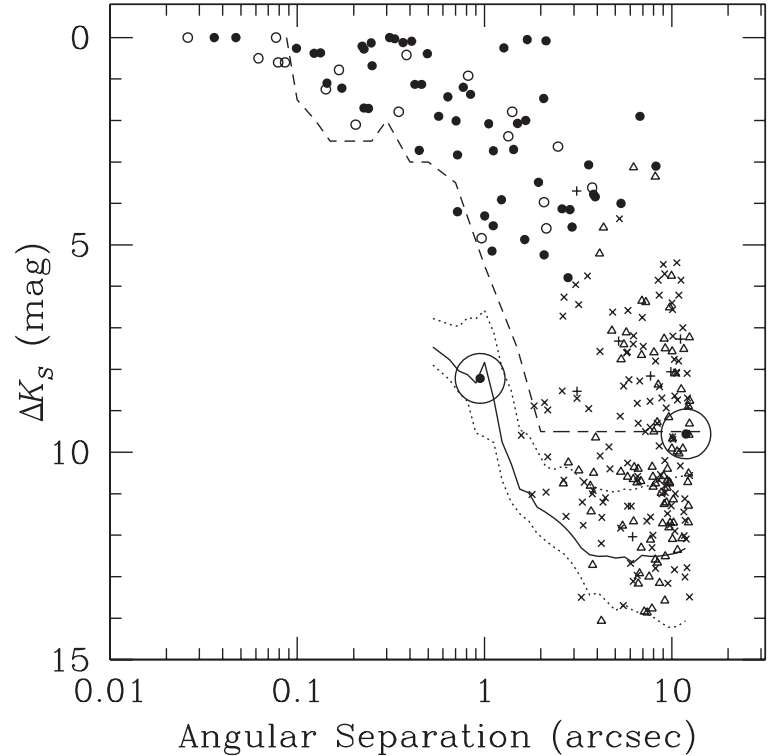

Figure 6. Magnitude difference $\Delta K_{S}$ vs. angular separation $\rho$ for all candidate companions discovered in the deep and shallow surveys. The various symbols denote: "๑"-astrometrically associated companions; " $x$ "- -astrometrically unassociated background stars; and for objects with insufficient astrometric data: "о"- companions associated based on their $J K_{S}$ photometry; "+"- - objects with $J K_{S}$ photometry inconsistent with association; " $\triangle$ "- undecided objects. The encircled points show the two brown dwarf companions from the survey: HD 49197B (at $\rho=0^{\prime \prime} .95$ ) and HD 203030B (at $\rho=11^{\prime \prime} .92$ ). Detection limits for the shallow (dashed line) and deep (solid and dotted lines) components of the survey are also shown. The solid line shows the median contrast $\Delta K_{S}$ of the deep survey, while the dotted lines delimit the 10-90 percentile region (see Figure 5(a)). Binaries with separations smaller than the PALAO $K_{S}$-band diffraction limit $\left(0{ }^{\prime \prime} 10\right)$ were resolved only at the $J$ band. Correspondingly, the plotted magnitude difference for these companions is the one at $J$.

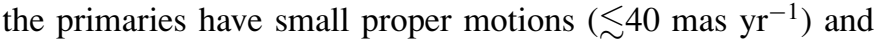
the images contain multiple background stars because of the low galactic latitude $\left(5^{\circ}<|b|<25^{\circ}\right)$.

\subsubsection{NIR CAMD and Background Object Density}

Systems with bright $\left(\Delta K_{S}<5\right.$ mag) close-in candidate secondaries often lacked dual-epoch astrometry in our survey. Such systems were given lower priority in follow-up observations be- 
Table 9

Candidate Companions in the Shallow Sample

\begin{tabular}{|c|c|c|c|c|c|c|c|c|c|c|}
\hline Primary Star & $N_{C}$ & $\begin{array}{c}\rho \\
(\operatorname{arcsec}) \\
\end{array}$ & $\begin{array}{c}\theta \\
(\mathrm{deg}) \\
\end{array}$ & $\begin{array}{c}\Delta K_{S} \\
(\mathrm{mag})\end{array}$ & $\begin{array}{c}K_{S} \\
(\mathrm{mag})\end{array}$ & $\begin{array}{c}J-K_{S} \\
(\mathrm{mag})\end{array}$ & $\begin{array}{c}t_{0} \\
\text { (UT Date) } \\
\end{array}$ & Tel. & Assoc. & Ref. \\
\hline HD 224873 & 1 & $1.268 \pm 0.002$ & $171.44 \pm 0.12$ & $0.25 \pm 0.02$ & $7.57 \pm 0.03$ & $0.50 \pm 0.05$ & 2002 Aug 31 & $\mathrm{P}$ & yes(a) & WDS \\
\hline HD 9472 & 1 & $2.793 \pm 0.025$ & $343.69 \pm 0.30$ & $5.79 \pm 0.09$ & $11.83 \pm 0.09$ & $1.04 \pm 0.14$ & 2002 Nov 18 & $\mathrm{P}$ & yes(a) & \\
\hline RE J0137+18A & 1 & $1.691 \pm 0.006$ & $24.60 \pm 0.12$ & $0.05 \pm 0.01$ & $7.49 \pm 0.02$ & $0.76 \pm 0.03$ & $2002 \operatorname{Jan} 31$ & $\mathrm{P}$ & yes(a) & WDS \\
\hline HD 13531 & 1 & $0.717 \pm 0.003$ & $16.79 \pm 0.43$ & $4.20 \pm 0.08$ & $9.88 \pm 0.08$ & $1.04 \pm 0.15$ & 2002 Aug 28 & $\mathrm{P}$ & yes(a) & \\
\hline \multirow[t]{2}{*}{ 1RXS J025223.5+372914 } & 1 & $0.637 \pm 0.003$ & $91.28 \pm 0.28$ & $1.43 \pm 0.08$ & $10.77 \pm 0.08$ & $0.67 \pm 0.12$ & 2003 Sep 21 & $\mathrm{P}$ & yes(a) & \\
\hline & 2 & $5.255 \pm 0.016$ & $76.85 \pm 0.18$ & $4.37 \pm 0.09$ & $13.45 \pm 0.09$ & $0.50 \pm 0.13$ & 2003 Sep 21 & $\mathrm{P}$ & no(a) & \\
\hline \multirow[t]{2}{*}{ 2RE J0255+474 } & 1 & $2.131 \pm 0.004$ & $272.63 \pm 0.16$ & $0.08 \pm 0.05$ & $7.29 \pm 0.06$ & $0.72 \pm 0.08$ & 2002 Feb 28 & $\mathrm{P}$ & yes(a) & WDS \\
\hline & 2 & $11.469 \pm 0.033$ & $46.40 \pm 0.11$ & $7.00 \pm 0.10$ & $14.21 \pm 0.10$ & $0.57 \pm 0.14$ & 2002 Feb 28 & $\mathrm{P}$ & no(a) & \\
\hline \multirow[t]{3}{*}{ HD 18940} & 1 & $0.167 \pm 0.002$ & $8.59 \pm 1.18$ & $0.78 \pm 0.03$ & $6.71 \pm 0.04$ & $0.51 \pm 0.08$ & 2002 Aug 29 & $\mathrm{P}$ & yes $(c)$ & Hip \\
\hline & 2 & $4.321 \pm 0.012$ & $207.38 \pm 0.12$ & $4.58 \pm 0.03$ & $10.08 \pm 0.04$ & $0.92 \pm 0.07$ & 2002 Aug 29 & $\mathrm{P}$ & $?$ & \\
\hline & 3 & $4.120 \pm 0.010$ & $203.78 \pm 0.13$ & $5.21 \pm 0.03$ & $10.71 \pm 0.04$ & $0.81 \pm 0.07$ & 2002 Aug 29 & $\mathrm{P}$ & $?$ & \\
\hline vB 1 & 1 & $2.470 \pm 0.006$ & $200.63 \pm 0.14$ & $2.63 \pm 0.03$ & $8.62 \pm 0.04$ & $0.91 \pm 0.06$ & 2002 Aug 29 & $\mathrm{P}$ & yes $(c)$ & WDS \\
\hline \multirow[t]{2}{*}{ HE 350} & 1 & $8.464 \pm 0.016$ & $109.22 \pm 0.14$ & $5.85 \pm 0.21$ & $15.11 \pm 0.21$ & $1.50 \pm 0.37$ & 2003 Sep 20 & $\mathrm{P}$ & no(a) & \\
\hline & 2 & $6.896 \pm 0.011$ & $38.37 \pm 0.19$ & $7.66 \pm 0.30$ & $16.92 \pm 0.30$ & & 2004 Oct 04 & $\mathrm{P}$ & $?$ & \\
\hline \multirow[t]{3}{*}{ HE 373} & 1 & $2.081 \pm 0.005$ & $193.77 \pm 0.18$ & $5.24 \pm 0.10$ & $14.59 \pm 0.10$ & $0.98 \pm 0.14$ & 2003 Sep 20 & $\mathrm{P}$ & yes(a) & MH04 \\
\hline & 2 & $11.598 \pm 0.031$ & $265.81 \pm 0.25$ & $7.51 \pm 0.30$ & $16.86 \pm 0.30$ & $\ldots$ & 2003 Sep 20 & $\mathrm{P}$ & $?$ & \\
\hline & 3 & $8.478 \pm 0.034$ & $55.82 \pm 0.22$ & $8.37 \pm 0.30$ & $17.72 \pm 0.30$ & $\ldots$ & 2003 Sep 20 & $\mathrm{P}$ & $?$ & \\
\hline HE 389 & 1 & $9.023 \pm 0.016$ & $133.30 \pm 0.12$ & $5.47 \pm 0.13$ & $14.96 \pm 0.13$ & $1.10 \pm 0.18$ & 2003 Sep 20 & $\mathrm{P}$ & no(a) & \\
\hline HE 696 & 1 & $0.448 \pm 0.001$ & $357.22 \pm 0.18$ & $2.72 \pm 0.08$ & $12.50 \pm 0.08$ & $0.70 \pm 0.12$ & 2003 Sep 20 & $\mathrm{P}$ & yes(a) & P02 \\
\hline \multirow[t]{2}{*}{ HE 935} & 1 & $0.026 \pm 0.025$ & $247.44 \pm 0.21$ & $0.00 \pm 0.20$ & $9.21 \pm 0.20$ & $0.40 \pm 0.28$ & 2003 Sep 20 & $\mathrm{P}$ & yes $(c)$ & $\mathrm{P} 02$ \\
\hline & 2 & $3.116 \pm 0.025$ & $109.45 \pm 0.21$ & $8.70 \pm 0.30$ & $17.16 \pm 0.30$ & $\ldots$ & 2003 Sep 20 & $\mathrm{P}$ & no(a) & \\
\hline \multirow[t]{2}{*}{ Н II 102} & 1 & $3.599 \pm 0.009$ & $213.29 \pm 0.14$ & $3.07 \pm 0.10$ & $11.72 \pm 0.10$ & $1.05 \pm 0.14$ & 2003 Sep 20 & $\mathrm{P}$ & yes(a) & B97 \\
\hline & 2 & $9.959 \pm 0.027$ & $240.21 \pm 0.16$ & $5.75 \pm 0.10$ & $14.40 \pm 0.10$ & $1.05 \pm 0.14$ & 2003 Sep 20 & $\mathrm{P}$ & $?$ & \\
\hline \multirow[t]{2}{*}{ 1RXS J034423.3+281224 } & 1 & $0.425 \pm 0.002$ & $202.20 \pm 0.10$ & $1.13 \pm 0.10$ & $8.62 \pm 0.10$ & $0.48 \pm 0.22$ & 2002 Nov 17 & $\mathrm{P}$ & yes(a) & WDS \\
\hline & 2 & $5.711 \pm 0.006$ & $313.30 \pm 0.12$ & $7.11 \pm 0.11$ & $14.27 \pm 0.11$ & & 2004 Oct 05 & $\mathrm{P}$ & $?$ & \\
\hline Н II 571 & 1 & $3.903 \pm 0.005$ & $66.10 \pm 0.08$ & $3.84 \pm 0.08$ & $13.07 \pm 0.08$ & $0.95 \pm 0.12$ & 2003 Dec 10 & $\mathrm{P}$ & yes(a) & B97, M92 \\
\hline Н II 1101 & 1 & $9.167 \pm 0.016$ & $104.93 \pm 0.12$ & $5.70 \pm 0.09$ & $14.46 \pm 0.09$ & $0.40 \pm 0.16$ & 2003 Dec 10 & $\mathrm{P}$ & no(a) & \\
\hline H II 1182 & 1 & $1.113 \pm 0.009$ & $219.69 \pm 0.26$ & $4.54 \pm 0.19$ & $13.48 \pm 0.19$ & $\ldots$ & 2003 Dec 10 & $\mathrm{P}$ & yes(a) & B97 \\
\hline H II 2106 & 1 & $0.240 \pm 0.010$ & $31.09 \pm 0.59$ & $1.71 \pm 0.12$ & $11.29 \pm 0.12$ & $\ldots$ & 2003 Dec 10 & $\mathrm{P}$ & yes(a) & B97 \\
\hline RX J0348.9+0110 & 1 & $0.047 \pm 0.007$ & $41.50 \pm 3.64$ & $0.00 \pm 0.05$ & $9.02 \pm 0.06$ & $\ldots$ & 2003 Dec 10 & $\mathrm{P}$ & yes(a) & \\
\hline Н II 2278 & 1 & $0.331 \pm 0.005$ & $179.20 \pm 0.32$ & $0.03 \pm 0.02$ & $9.57 \pm 0.03$ & & 2003 Dec 10 & $\mathrm{P}$ & yes(a) & B97 \\
\hline Н II 2881 & 1 & $0.099 \pm 0.005$ & $335.73 \pm 1.20$ & $0.26 \pm 0.09$ & $9.94 \pm 0.09$ & 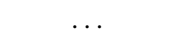 & 2003 Dec 10 & $\mathrm{P}$ & yes(a) & B97 \\
\hline HD 285281 & 1 & $0.770 \pm 0.001$ & $188.34 \pm 0.05$ & $1.20 \pm 0.10$ & $9.12 \pm 0.10$ & $0.66 \pm 0.14$ & 2002 Feb 01 & $\mathrm{P}$ & yes(a) & KL98 \\
\hline HD 284135 & 1 & $0.367 \pm 0.002$ & $253.23 \pm 0.21$ & $0.12 \pm 0.01$ & $8.58 \pm 0.02$ & & 2002 Jan 31 & $\mathrm{P}$ & yes(a) & WDS \\
\hline HD 281691 & 1 & $6.768 \pm 0.014$ & $138.91 \pm 0.13$ & $1.90 \pm 0.05$ & $10.30 \pm 0.06$ & $0.74 \pm 0.08$ & 2002 Nov 18 & $\mathrm{P}$ & yes(a) & KL98 \\
\hline HD 26182 & 1 & $0.818 \pm 0.002$ & $175.11 \pm 0.11$ & $0.92 \pm 0.08$ & $9.09 \pm 0.08$ & $0.42 \pm 0.11$ & 2003 Dec 10 & $\mathrm{P}$ & yes(c) & WDS \\
\hline HD 284266 & 1 & $0.569 \pm 0.006$ & $356.92 \pm 0.11$ & $1.90 \pm 0.10$ & $10.66 \pm 0.10$ & $0.89 \pm 0.41$ & 2002 Jan 31 & $\mathrm{P}$ & yes(a) & KL98 \\
\hline HD 26990 & 1 & $0.123 \pm 0.004$ & $163.56 \pm 1.40$ & $0.38 \pm 0.20$ & $6.81 \pm 0.20$ & $0.67 \pm 0.36$ & 2003 Dec 10 & $\mathrm{P}$ & yes(a) & \\
\hline vB 49 & 1 & $2.139 \pm 0.017$ & $256.86 \pm 0.16$ & $4.60 \pm 0.14$ & $11.40 \pm 0.14$ & & 2003 Jan 12 & $\mathrm{P}$ & yes $(c)$ & \\
\hline vB 52 & 1 & $1.115 \pm 0.002$ & $236.40 \pm 0.18$ & $2.73 \pm 0.06$ & $9.10 \pm 0.06$ & $0.85 \pm 0.08$ & 2003 Jan 12 & $\mathrm{P}$ & yes(a) & P98 \\
\hline vB 176 & 1 & $0.227 \pm 0.003$ & $307.06 \pm 0.38$ & $0.28 \pm 0.09$ & $7.67 \pm 0.09$ & $\ldots$ & 2003 Dec 10 & $\mathrm{P}$ & yes(a) & Hip \\
\hline vB 66 & 1 & $9.781 \pm 0.023$ & $248.88 \pm 0.11$ & $10.75 \pm 0.10$ & $16.91 \pm 0.10$ & $\ldots$ & 2002 Nov 17 & $\mathrm{P}$ & $?$ & \\
\hline vB 91 & 1 & $0.133 \pm 0.002$ & $172.98 \pm 2.79$ & $0.37 \pm 0.14$ & $7.72 \pm 0.14$ & 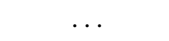 & 2003 Dec 10 & $\mathrm{P}$ & yes(a) & WDS \\
\hline vB 96 & 1 & $0.171 \pm 0.003$ & $264.05 \pm 0.78$ & $0.36 \pm 0.10$ & $7.41 \pm 0.10$ & $\ldots$ & 2003 Dec 10 & $\mathrm{P}$ & yes(p) & P98 \\
\hline RX J0434.3+0226 & 1 & $1.340 \pm 0.022$ & $271.76 \pm 0.30$ & $2.38 \pm 0.05$ & $11.99 \pm 0.06$ & $0.77 \pm 0.11$ & 2003 Jan 12 & $\mathrm{P}$ & yes(c) & \\
\hline vB 106 & 1 & $7.230 \pm 0.012$ & $76.50 \pm 0.44$ & $9.50 \pm 0.30$ & $15.94 \pm 0.30$ & 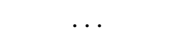 & 2003 Dec 10 & $\mathrm{P}$ & no(a) & \\
\hline HD 282346 & 1 & $0.461 \pm 0.001$ & $272.14 \pm 0.18$ & $1.13 \pm 0.04$ & $8.91 \pm 0.04$ & $0.61 \pm 0.17$ & 2002 Nov 18 & $\mathrm{P}$ & yes(a) & Hip \\
\hline vB 142 & 1 & $6.070 \pm 0.013$ & $123.82 \pm 0.16$ & $11.30 \pm 0.20$ & $18.04 \pm 0.20$ & & 2002 Nov 17 & $\mathrm{P}$ & no(a) & \\
\hline 1RXS J051111.1+281353 & 1 & $0.495 \pm 0.001$ & $211.51 \pm 0.10$ & $0.39 \pm 0.04$ & $8.77 \pm 0.05$ & $0.69 \pm 0.07$ & 2002 Feb 28 & $\mathrm{P}$ & yes(a) & \\
\hline \multirow[t]{2}{*}{ HD 36869} & 1 & $8.230 \pm 0.014$ & $152.30 \pm 0.12$ & $3.10 \pm 0.35$ & $9.95 \pm 0.35$ & $0.93 \pm 0.35$ & 2003 Jan 14 & $\mathrm{P}$ & yes(a) & \\
\hline & 2 & $8.043 \pm 0.016$ & $249.72 \pm 0.20$ & $7.59 \pm 0.15$ & $14.44 \pm 0.15$ & & 2003 Jan 14 & $\mathrm{P}$ & $?$ & \\
\hline HD 61994 & 1 & $5.210 \pm 0.008$ & $77.00 \pm 0.08$ & $7.32 \pm 0.13$ & $12.67 \pm 0.13$ & $0.28 \pm 0.30$ & 2002 Nov 18 & $\mathrm{P}$ & no(c) & \\
\hline HD 69076 & 1 & $1.232 \pm 0.005$ & $101.06 \pm 0.11$ & $3.91 \pm 0.05$ & $10.38 \pm 0.05$ & $1.04 \pm 0.21$ & 2002 Nov 18 & $\mathrm{P}$ & yes(a) & \\
\hline HD 71974 & 1 & $0.383 \pm 0.014$ & $87.34 \pm 0.63$ & $0.42 \pm 0.05$ & $6.45 \pm 0.06$ & $0.35 \pm 0.21$ & 2002 Mar 03 & $\mathrm{P}$ & yes(c) & S99 \\
\hline HD 72760 & 1 & $0.964 \pm 0.007$ & $215.08 \pm 0.38$ & $4.84 \pm 0.01$ & $10.28 \pm 0.02$ & $1.01 \pm 0.04$ & 2002 Nov 16 & $\mathrm{P}$ & yes(c) & \\
\hline HD 77407 & 1 & $1.659 \pm 0.004$ & $353.36 \pm 0.04$ & $2.00 \pm 0.10$ & $7.60 \pm 0.10$ & $0.90 \pm 0.14$ & 2002 Jan 31 & $\mathrm{P}$ & yes(a) & M04 \\
\hline HD 78899 & 1 & $8.174 \pm 0.013$ & $75.76 \pm 0.12$ & $3.36 \pm 0.08$ & $9.17 \pm 0.08$ & $0.79 \pm 0.13$ & 2003 Dec 09 & $\mathrm{P}$ & $?$ & \\
\hline \multirow[t]{2}{*}{ HD 91962} & 1 & $0.842 \pm 0.003$ & $176.00 \pm 0.11$ & $1.37 \pm 0.06$ & $7.03 \pm 0.06$ & $0.73 \pm 0.13$ & 2002 Mar 02 & $\mathrm{P}$ & yes(a) & WDS \\
\hline & 2 & $0.142 \pm 0.004$ & $56.17 \pm 1.76$ & $1.25 \pm 0.11$ & $6.94 \pm 0.11$ & $0.98 \pm 0.19$ & 2003 May 10 & $\mathrm{P}$ & yes(c) & \\
\hline HD 99565 & 1 & $0.408 \pm 0.001$ & $6.13 \pm 0.18$ & $0.09 \pm 0.05$ & $6.55 \pm 0.05$ & $0.48 \pm 0.06$ & 2003 Jan 11 & $\mathrm{P}$ & yes(a) & WDS \\
\hline HD 108799 & 1 & $2.070 \pm 0.006$ & $338.46 \pm 0.09$ & $1.47 \pm 0.02$ & $6.30 \pm 0.03$ & $0.80 \pm 0.06$ & 2003 May 10 & $\mathrm{P}$ & yes(a) & WDS \\
\hline HD 108944 & 1 & $1.941 \pm 0.006$ & $345.48 \pm 0.18$ & $3.49 \pm 0.02$ & $9.56 \pm 0.03$ & $0.85 \pm 0.09$ & 2002 Mar 03 & $\mathrm{P}$ & yes(a) & \\
\hline HD 112196 & 1 & $1.501 \pm 0.001$ & $55.52 \pm 0.09$ & $2.07 \pm 0.01$ & $7.77 \pm 0.02$ & $0.73 \pm 0.04$ & 2002 Feb 01 & $\mathrm{P}$ & yes(a) & \\
\hline HD 115043 & 1 & $1.639 \pm 0.003$ & $358.61 \pm 0.05$ & $4.87 \pm 0.08$ & $10.22 \pm 0.08$ & $0.86 \pm 0.12$ & 2003 Dec 09 & $\mathrm{P}$ & yes(a) & L05 \\
\hline HD 129333 & 1 & $0.717 \pm 0.009$ & $172.77 \pm 0.11$ & $2.83 \pm 0.05$ & $8.82 \pm 0.05$ & $0.81 \pm 0.07$ & 2003 Jan 11 & $\mathrm{P}$ & yes(a) & DM91,MH04 \\
\hline
\end{tabular}


Table 9

(Continued)

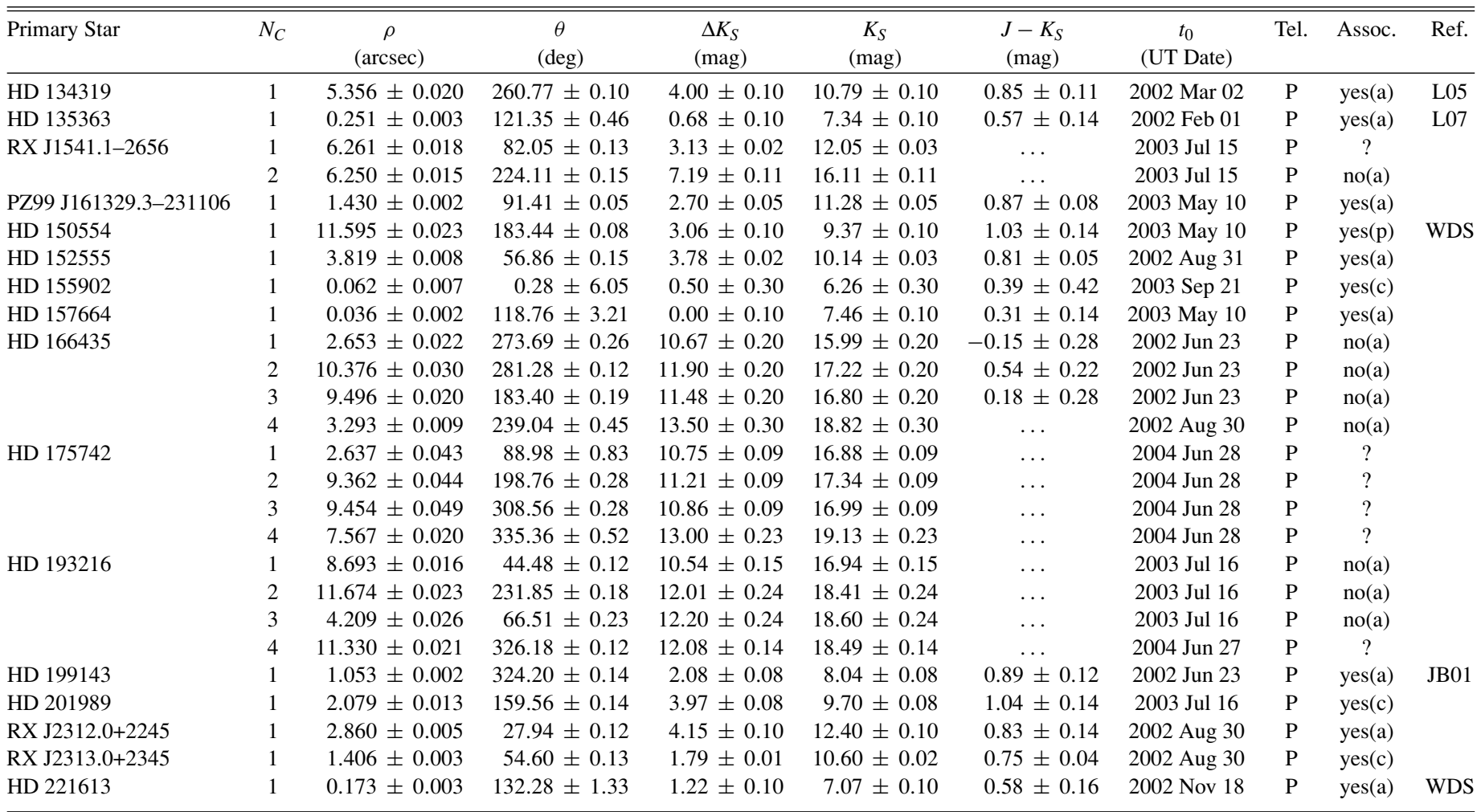

References. B97: Bouvier et al. (1997); DM91: Duquennoy \& Mayor (1991); Hip: Perryman et al. (1997, Hipparcos); JB01: Jayawardhana \& Brandeker (2001); KL98: Kohler \& Leinert (1998); L05: Lowrance et al. (2005); L07: Lafrenière et al. (2007); M04: Mugrauer et al. (2004); M92: Mermilliod et al. (1992); MH04: Metchev \& Hillenbrand (2004); P02: Patience et al. (2002); P98: Patience et al. (1998); S99: Söderhjelm (1999); WDS: Mason et al. (2001; Washington Double Star Catalog, and references therein).

(This table is also available in a machine-readable form in the online journal)

cause the companions were considered to be stellar and almost certainly bound. Multiepoch astrometric analysis was inapplicable in these cases. However, the candidate stellar secondaries in these systems were bright enough to be seen in the shallow noncoronagraphic $J H K_{S}$ exposures of our targets. Hence, for the majority of the candidate stellar systems lacking astrometric confirmation, physical association could be estimated based on the NIR colors and expected absolute magnitudes of the components.

In evaluating the association of a candidate companion based on its NIR photometry, we placed it on a $J-K_{S}$ versus $M_{K_{S}}$ CAMD and checked whether it laid on the same isochrone as the primary. In the substellar regime, especially near the $\mathrm{L} / \mathrm{T}$ transition $\left(12<M_{K}<14\right)$ where the isochrones are not well constrained, we relied on the empirical main sequence as traced by nearby M-T dwarfs (Leggett et al. 2002, Reid et al. 2004) with known parallaxes (from Dahn et al. 2002, Vrba et al. 2004). All candidate companions with available $J$-band photometry for which the astrometry was inconclusive had their physical associations with their candidate primaries evaluated in this manner (Figure 7). To limit the probability of misclassifying field stars as bona fide companions, positive associations were adopted only for candidate companions within a $5^{\prime \prime}$ radius field of interest from the primary.

This approach was successful mostly for stellar-mass companions bluer than $J-K_{S}=0.8 \mathrm{mag}$, that is, earlier than spectral type M0. The main sequence for redder M0-M6 dwarfs is nearly degenerate in $J-K_{S}$ over nearly 4 mag in $M_{K_{S}}$ (see

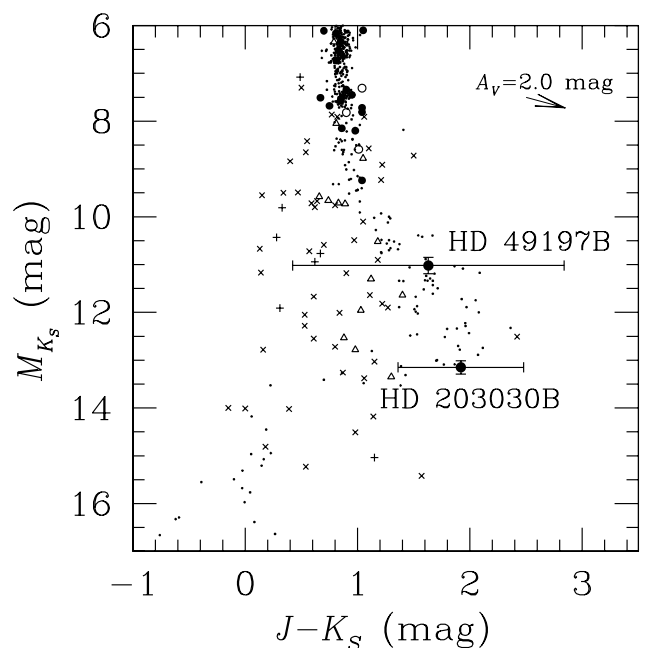

Figure 7. $M_{K_{S}}$ vs. $J-K_{S}$ color-magnitude diagram of candidate companions for which $J$-band photometry was obtained. The symbols are the same as in Figure 6. The additional small dots denote M0-T8 dwarfs with parallaxes from (Dahn et al. 2002) and (Vrba et al. 2004). The points with errorbars represent the two confirmed brown dwarf companions from our survey. The errorbars on HD $203030 \mathrm{~B}$ are representative of the photometric precision for the faintest $(J \gtrsim 18$ mag) objects in the survey. Brighter objects typically had $J-K_{S}$ errors $<0.3$ mag, except for the large $J-K_{S}$ uncertainty of HD 49197B, which is unique because of its relative faintness $(\Delta J=9.6 \mathrm{mag})$ and proximity $\left(\rho=00^{\prime} .95\right)$ to the primary. The vector in the upper right corresponds to $A_{V}=2 \mathrm{mag}$ of visual extinction, equivalent to a distance of $\sim 3 \mathrm{kpc}$, or a distance modulus of $12 \mathrm{mag}$ along the galactic plane. 
Figure 7) and does not allow reliable association estimates from the absolute $K_{S}$ magnitude. At even later spectral types, potentially representative of young brown dwarf companions, the higher photometric uncertainties and the larger empirical color scatter at substellar masses prevented the conclusive determination of physical association in all but a handful of faint projected companions. $H$-band photometry, where available, did not improve the analysis because of the smaller wavelength range sampled by the $H-K_{S}$ versus $J-K_{S}$ colors. Thus, no candidate substellar companions were confirmed through NIR photometry. However, a few could be rejected.

In addition to using NIR colors, it was also possible to obtain a probabilistic estimate of the physical association of a candidate companion with its corresponding primary by comparing the number of detected objects within the $12^{\prime \prime} .5$ survey radius to the surface density of stars at the relevant galactic coordinates down to the limiting magnitude of the survey. Because of the lack of large-area deep ( $K_{S} \lesssim 20 \mathrm{mag}$ ) NIR survey data, we limited this type of analysis only to candidate companions in the shallow survey. Although the depth of the shallow survey varied depending on the use of the ND filter at Palomar, it was roughly comparable to the $99 \%$ completeness limit of the 2MASS catalog: $K_{S}<14.3 \mathrm{mag}$ in unconfused regions of the sky. Therefore, for all candidate companions brighter than $K_{S}=14.3 \mathrm{mag}$, an empirical estimate of the association probability was possible based on 2MASS. Given that the faintest primaries in the sample have $K_{S}$ magnitudes of 9.6, such a probabilistic analysis could be performed on all candidate companions with $\Delta K_{S} \leqslant 4.7 \mathrm{mag}$.

To estimate the contamination from $K_{S} \leqslant 14.3 \mathrm{mag}$ field stars, we counted the number of 2 MASS objects within a $5^{\prime}$ radius circular area offset by $12^{\prime}$ from each sample star (to avoid bright-star artifacts), and from that we obtained the expected number of background objects in the $5^{\prime \prime}$ radius field of interest. We used this as an estimate of the purely geometrical chance alignment probability (CAP):

$$
\mathrm{CAP}=\left(\text { number of } 2 \mathrm{MASS} \text { sources within } 5^{\prime}\right) \times \frac{\pi\left(5^{\prime \prime}\right)^{2}}{\pi\left(5^{\prime}\right)^{2}} \text {. }
$$

Table 10 lists the separations, $\Delta K_{S}$ and $K_{S}$ magnitudes, and the CAPs for all sample stars with color companions (i.e., the ones with "yes(c)" entries in Tables 8 and 9). Most color companions have chance alignment probabilities $\lesssim 2 \%$, with the exceptions of HD 155902B and HE 935B. However, both of these are very close $\left(<0^{\prime \prime} 1\right)$ to their candidate primaries, and are thus almost certainly physical companions. These two systems are in fact below the resolution limits of the 95 mas PALAO $K_{S^{-}}$ band PSF. Their binarity was only appreciated from PALAO $J$-band images, where the PSF is 50 mas wide. The ensemble probability of at least one of the 17 color companions being a false positive is $35 \%$, or $18 \%$ if HD 155902 and HE 935 are excluded.

\subsubsection{Source Extent}

Any bona fide companions to our sample stars were expected to be point sources. Apparent source extension could, in principle, be used to exclude background galaxies seen in projection. However, the determination of source extent is not a trivial task when the quality of the AO correction and, hence, the size and shape of the PSF change throughout the course of a single night depending on guide star brightness and on atmospheric stability. In addition, anisoplanatism may radially elongate point-source
PSFs far away from the central AO guide star. Therefore, departures from the nominal, diffraction-limited PSF size and from a centrally symmetric PSF shape were regarded with caution. These were used to classify an object as an extended source only when they were in disagreement with the size and radial behavior of the profiles of other point sources in the same image, if such were present.

\subsubsection{Physical Association Summary}

Using the above criteria, we were able to determine the physical association for 199 of the 287 companion candidates. The proper motion criterion (Section 5.2.1) was used to establish the majority of associations or nonassociations: 170 out of $199(85.4 \%)$. These included 58 bona fide common proper motion companions and 112 noncommon proper motion background objects seen in projection. An additional apparent common proper motion companion to ScoPMS 214 was rejected as a bona fide companion based on follow-up spectroscopy (Section 6.3).

The CAMD and chance alignment criteria (Section 5.2.2) are not conclusive in proving physical association. They were invoked only when astrometric follow-up was not obtained or the proper motion data were ambiguous, and when additional $J$-band images were taken (Section 5.2.2). These criteria were used in tandem to establish the physical association of candidate stellar companions with high fidelity in the 18 cases listed in Table 10. The CMD criterion alone was used in seven cases to exclude background interlopers.

The source extent criterion (Section 5.2.3) was used to weed out faint galaxies, which may otherwise have red NIR colors, partially due to line-of-sight (LOS) extinction, and may thus pose as candidate substellar objects for the preceding criterion. This criterion was applied in four cases.

None of the above criteria were applicable to 87 candidate companions $(30.3 \%$ of the total) that remained "undecided." The vast majority of these were faint objects in the fields of distant $(>100 \mathrm{pc})$ stars with small apparent proper motions $\left(<50\right.$ mas yr $\left.^{-1}\right)$, often at low galactic latitudes $\left(b<15^{\circ}\right)$. These were often discovered only in follow-up deeper imaging with Keck, and thus lack the full time span of astrometric observations. Judging by the large number of such candidate companions per star, and based on expectations of the background star contamination rate at low galactic latitudes, probably none of these candidate companions are associated. Throughout the rest of the analysis, we shall assume that all 87 of the undecided candidates are unassociated field stars.

\section{SURVEY RESULTS}

Preliminary results from the survey were already published in Metchev \& Hillenbrand $(2004,2006)$, including the discovery of two brown dwarf companions, HD 49197B and HD 203030B. In this paper, we report the results from the full survey. We found no more substellar companions in our sample. We summarize the findings on the two previously discovered substellar companions in Section 6.1. We also report 21 new stellar companions, in addition to three (HD 129333B, HE 373B, and RX J0329.1+0118B) already announced in Metchev \& Hillenbrand (2004). Four of the newly discovered stellar companions, HD 9472B, HE 373B, HD 31950B, PZ99 J161329.3-231106B, in addition to the previously announced RX J0329.1+0118B have masses of only $\approx 0.1 M_{\odot}$, and reside in very low mass ratio ( $\left.q=M_{2} / M_{1} \approx 0.1\right)$ systems. The results on the stellar companions are detailed in Section 6.2. 
Table 10

Color Companions and Their Chance Alignment Probabilities

\begin{tabular}{|c|c|c|c|c|c|}
\hline Companion to & $N_{C}$ & $\begin{array}{c}\rho \\
(\operatorname{arcsec})\end{array}$ & $\begin{array}{c}\Delta K_{S} \\
(\mathrm{mag})\end{array}$ & $\begin{array}{c}K_{S} \\
(\mathrm{mag})\end{array}$ & $\begin{array}{l}\text { CAP } \\
(\%)\end{array}$ \\
\hline HD 15526 & 1 & 0.0770 & 0.00 & $8.01 \pm 0.10$ & 0.8 \\
\hline RX J0258.4+2947 & 1 & 0.086 & 0.60 & $10.15 \pm 0.30$ & 2.4 \\
\hline HD 155902 & 1 & 0.0620 & 0.50 & $5.73 \pm 0.10$ & 14.3 \\
\hline HD 18940 & 1 & 0.1670 & 0.78 & $6.28 \pm 0.10$ & 1.5 \\
\hline HD 201989 & 1 & 2.0790 & 3.97 & $9.70 \pm 0.08$ & 0.9 \\
\hline vB 1 & 1 & 2.4700 & 2.63 & $8.62 \pm 0.10$ & 0.9 \\
\hline HD 245567 & 1 & 0.3480 & 1.79 & $9.38 \pm 0.10$ & 0.8 \\
\hline HD 26182 & 1 & 0.8180 & 0.92 & $8.71 \pm 0.10$ & 2.3 \\
\hline vB 49 & 1 & 2.1390 & 4.56 & $11.36 \pm 0.10$ & 1.3 \\
\hline HD 71974 & 1 & 0.3830 & 0.42 & $5.89 \pm 0.10$ & 1.7 \\
\hline HD 72760 & 1 & 0.9640 & 4.84 & $10.26 \pm 0.10$ & 0.9 \\
\hline HD 91962 & 2 & 0.1420 & 1.25 & $6.64 \pm 0.10$ & 0.9 \\
\hline HE 935 & 1 & 0.0260 & 0.30 & $8.76 \pm 0.10$ & 6.7 \\
\hline RX J0329.1+0118 & 1 & 3.7610 & 3.62 & $12.82 \pm 0.10$ & 0.7 \\
\hline RX J0354.4+0535 & 2 & 0.2050 & 2.10 & $10.77 \pm 0.10$ & 1.4 \\
\hline RX J0434.3+0226 & 1 & 1.3400 & 2.38 & $11.88 \pm 0.10$ & 1.2 \\
\hline RX J2313.0+2345 & 1 & 1.4060 & 1.79 & $10.41 \pm 0.10$ & 1.4 \\
\hline ScoPMS 27 & 1 & 0.0790 & 0.60 & $8.64 \pm 0.10$ & 0.7 \\
\hline
\end{tabular}

Notes. Most columns headings are as for Tables 8 and 9. CAP is the chance alignment probability from Equation (1) in Section 5.2.2.

An apparent proper motion companion to the star ScoPMS 214, considered to be a brown dwarf based on its apparent $K_{S}$ magnitude in Metchev (2006), was found to most probably be an unassociated foreground M star after spectroscopic follow-up. This companion, ScoPMS 214"B," is discussed in Section 6.3 as an example of a pathological case where spectroscopic analysis argues against the physical association in an apparent common proper motion system.

Independent of the unbiased survey for substellar companions, we also observed and established the physical association of a previously known (Bouvier et al. 1997) candidate companion to $\mathrm{H}$ II 1348 . The estimated mass of H II $1348 \mathrm{~B}$ is near the limit for sustained hydrogen burning. Because of our deliberate inclusion of H II 1348 in our observing program based on known binarity, it is excluded from the present analysis. H II 1348B will be the subject of a future publication.

\subsection{Brown Dwarf Companions}

Both brown dwarf companions, HD 49197B and HD $203030 \mathrm{~B}$, were found in the 100 star deep survey. The observed photometric and astrometric properties of the two and their inferred masses are listed in Table 11 alongside those of the stellar secondaries observed in our survey. The spectral types of HD 49197B and HD 203030B are L4 \pm 1 and L7.5 \pm 0.5 , respectively, and their masses are estimated at $0.060_{-0.020}^{+0.012} M_{\odot}$ (Metchev \& Hillenbrand 2004) and $0.023_{-0.011}^{+0.008} M_{\odot}$ (Metchev \& Hillenbrand 2006).

Because of their association with main-sequence stars, the ages of HD 49197B and HD 203030B are known with relative certainty. Their moderate youth (250-500 Myr) makes them valuable as benchmarks for substellar properties at $\log g \approx 5$ surface gravities, $\sim 0.5$ dex lower than the gravities expected of 3-5 Gyr old brown dwarfs in the field.

At the time of its discovery, HD 49197B was only the fifth known L dwarf younger than $1 \mathrm{Gyr}$. At a projected separation of only $43 \mathrm{AU}$ from its host star, HD 49197B was also one of the closest-in resolved substellar companions, second only to HR 7672B (14 AU; Liu et al. 2002). Both HR 7672B and HD
49197B provided early indication that the brown dwarf desert may not extend much outside of $3 \mathrm{AU}$ (Metchev \& Hillenbrand 2004).

HD 203030B was the first young brown dwarf with a spectral type unambiguously as late as the L/T transition. Its surprising underluminosity, by $\approx 0.5 \mathrm{dex}$ compared to theoretical predictions for $\sim 1400 \mathrm{~K}$ brown dwarfs at its age, indicated that its effective temperature was $\approx 200 \mathrm{~K}$ cooler (i.e., $\approx 1200 \mathrm{~K}$ ) than expected at the L/T transition. That is, the spectrophotometric properties of HD 203030B indicated that either the effective temperature at the L/T transition had a heretofore unappreciated dependence on surface gravity, or that the entire population of field substellar objects had their effective temperatures and ages significantly overestimated.

In fact, underluminosity and effective temperatures less than $1400 \mathrm{~K}$ are inferred for all known substellar companions near the L/T transition (Metchev \& Hillenbrand 2006), including both the recently-discovered T2.5 dwarf HN PegB (Luhman et al. 2007a) and the presumed planetary mass 2MASS J1207334393254B (L5-L9; Chauvin et al. 2005a; Mohanty et al. 2007). With the mean ages of their respective primaries ranging between $8 \mathrm{Myr}$ and $2 \mathrm{Gyr}$, all six known L/T transition companions (GJ 584C, Kirkpatrick et al. 2001; GJ 337CD, Wilson et al. 2001; Burgasser et al. 2005; 2MASS J1207334393254B, Chauvin et al. 2005a; HD 203030B, Metchev \& Hillenbrand 2006; HN PegB, Luhman et al. 2007a) are likely younger than the inferred 2.9 Gyr mean age of L/T transition dwarfs in the solar neighborhood (Allen et al. 2005). Therefore, the theory may indeed be overestimating the ages of field brown dwarfs by a factor of at least 1.5. This hypothesis has now been independently reinforced by the first measurement of the dynamical mass of a binary field T dwarf. Liu et al. (2008) found that the components of the T5.0+T5.5 dwarf binary 2MASS J15344984-2952274AB are about $100 \mathrm{~K}$ cooler than derived for similar field objects - a fact that they interpret as evidence for a factor of $\approx 6 \pm 3$ overestimate in the adopted ages of field brown dwarfs. Future high-contrast imaging and astrometric observations and discoveries of benchmark brown dwarfs with 
Table 11

New and Confirmed Secondaries

\begin{tabular}{|c|c|c|c|c|c|c|c|}
\hline \multirow[t]{2}{*}{ Companion } & \multirow{2}{*}{$\begin{array}{c}M_{K_{S}} \\
(\mathrm{mag})\end{array}$} & \multicolumn{2}{|c|}{$\begin{array}{l}\text { Projected } \\
\text { Separation }\end{array}$} & \multirow{2}{*}{$\begin{array}{c}M_{2} \\
\left(M_{\odot}\right)\end{array}$} & \multirow[t]{2}{*}{$q$} & \multirow[t]{2}{*}{ Sample $^{\mathrm{a}}$} & \multirow[t]{2}{*}{ Ref. } \\
\hline & & $(\operatorname{arcsec})$ & $(\mathrm{AU})$ & & & & \\
\hline HD 224873B & $4.12 \pm 0.22$ & 1.27 & 62.13 & 0.84 & 0.98 & $\mathrm{AD}_{30}$ & WDS \\
\hline HD 9472B & $9.24 \pm 0.11$ & 2.79 & 92.17 & 0.11 & 0.10 & & \\
\hline RE J0137+18B & $3.46 \pm 0.27$ & 1.69 & 108.22 & 0.63 & 0.97 & $\mathrm{AD}_{30}$ & WDS \\
\hline HD 13531B & $7.81 \pm 0.12$ & 0.72 & 18.64 & 0.19 & 0.20 & $\mathrm{AD}_{30}$ & \\
\hline HD 15526B & $3.63 \pm 0.54$ & 0.08 & 8.16 & 0.90 & 1.00 & $\mathrm{AD}$ & \\
\hline 1RXS J025223.5+372914B & $4.62 \pm 1.09$ & 0.64 & 108.29 & 0.71 & 0.66 & $\mathrm{AD}_{30}$ & \\
\hline 2RE J0255+474B & $3.80 \pm 1.09$ & 2.13 & 106.55 & 0.91 & 0.99 & $\mathrm{AD}_{30}$ & WDS \\
\hline RX J0258.4+2947B & $5.15 \pm 1.13$ & 0.09 & 8.60 & 0.59 & 0.78 & $\mathrm{AD}$ & \\
\hline HD 18940B & $4.05 \pm 0.08$ & 0.17 & 5.68 & 0.86 & 0.83 & & Hip \\
\hline vB $1 \mathrm{~B}$ & $5.45 \pm 0.05$ & 2.47 & 106.46 & 0.56 & 0.47 & & WDS \\
\hline HE 373B & $8.20 \pm 0.16$ & 2.08 & 395.39 & 0.10 & 0.08 & & MH04 \\
\hline HE 696B & $6.11 \pm 0.15$ & 0.45 & 85.12 & 0.39 & 0.38 & & P02 \\
\hline RX J0329.1+0118B & $7.82 \pm 1.09$ & 3.76 & 376.10 & 0.11 & 0.12 & $\mathrm{AD}_{30}$ & MH04 \\
\hline HE 935B & $2.82 \pm 0.24$ & 0.03 & 4.94 & 1.20 & 1.00 & & P02 \\
\hline H II 102B & $6.10 \pm 0.14$ & 3.60 & 478.67 & 0.43 & 0.39 & $\mathrm{AD}_{30}$ & B97 \\
\hline 1RXS J034423.3+281224B & $5.17 \pm 0.20$ & 0.43 & 20.83 & 0.57 & 0.71 & $\mathrm{AD}$ & WDS \\
\hline Н II $571 \mathrm{~B}$ & $7.45 \pm 0.13$ & 3.90 & 519.10 & 0.20 & 0.20 & & B97, M92 \\
\hline H II 1182B & $7.86 \pm 0.21$ & 1.11 & 148.03 & 0.15 & 0.14 & & B97 \\
\hline Н II $2106 \mathrm{~B}$ & $5.67 \pm 0.15$ & 0.24 & 31.92 & 0.51 & 0.59 & & B97 \\
\hline RX J0348.9+0110B & $4.02 \pm 1.09$ & 0.05 & 4.70 & 0.87 & 1.00 & & \\
\hline H II $2278 \mathrm{~B}$ & $3.95 \pm 0.10$ & 0.33 & 44.02 & 0.89 & 0.99 & & B97 \\
\hline H II $2881 \mathrm{~B}$ & $4.32 \pm 0.13$ & 0.10 & 13.17 & 0.79 & 0.92 & & B97 \\
\hline RX J0354.4+0535B & $5.92 \pm 1.10$ & 0.21 & 20.50 & 0.48 & 0.50 & $\mathrm{AD}$ & \\
\hline HD 285281B & $5.67 \pm 0.20$ & 0.77 & 37.73 & 0.19 & 0.42 & $\mathrm{AD}_{30}$ & KL98 \\
\hline HD 284135B & $2.85 \pm 1.09$ & 0.37 & 51.38 & 0.98 & 0.93 & $\mathrm{AD}$ & WDS \\
\hline HD 281691B & $4.57 \pm 1.09$ & 6.77 & 947.52 & 0.68 & 0.60 & $\mathrm{AD}_{30}$ & KL98 \\
\hline HD 26182B & $4.09 \pm 1.09$ & 0.82 & 81.80 & 0.79 & 0.72 & $\mathrm{AD}_{30}$ & WDS \\
\hline HD 284266B & $4.93 \pm 1.09$ & 0.57 & 79.66 & 0.43 & 0.38 & $\mathrm{AD}_{30}$ & KL98 \\
\hline HD 26990B & $4.09 \pm 0.24$ & 0.12 & 4.31 & 0.84 & 0.91 & & \\
\hline vB 49B & $7.60 \pm 0.15$ & 2.14 & 122.99 & 0.22 & 0.19 & & \\
\hline vB 52B & $5.84 \pm 0.07$ & 1.12 & 49.95 & 0.49 & 0.45 & & P98 \\
\hline vB 176B & $4.26 \pm 0.10$ & 0.23 & 10.90 & 0.80 & 0.99 & & Hip \\
\hline vB 91B & $4.41 \pm 0.14$ & 0.13 & 6.10 & 0.76 & 0.94 & & WDS \\
\hline vB 96B & $4.12 \pm 0.11$ & 0.17 & 7.76 & 0.84 & 0.97 & & P98 \\
\hline RX J0434.3+0226B & $5.96 \pm 0.33$ & 1.34 & 215.74 & 0.38 & 0.39 & $\mathrm{AD}_{30}$ & \\
\hline HD 282346B & $4.65 \pm 0.43$ & 0.46 & 32.73 & 0.70 & 0.73 & $\mathrm{AD}$ & Hip \\
\hline HD 31950B & $7.51 \pm 1.09$ & 2.60 & 259.60 & 0.13 & 0.12 & $\mathrm{AD}_{30}$ & \\
\hline 1RXS J051111.1+281353B & $2.73 \pm 0.20$ & 0.50 & 68.81 & 0.80 & 0.76 & $\mathrm{AD}$ & \\
\hline HD 36869B & $5.66 \pm 0.72$ & 8.23 & 592.56 & 0.36 & 0.30 & $\mathrm{AD}_{30}$ & \\
\hline HD 245567B & $4.19 \pm 0.39$ & 0.35 & 41.41 & 0.30 & 0.27 & $\mathrm{AD}$ & \\
\hline HD 49197B & $11.02 \pm 0.17$ & 0.95 & 42.66 & 0.06 & 0.05 & $\mathrm{AD}_{30}$ & MH04 \\
\hline HD 69076B & $7.72 \pm 0.08$ & 1.23 & 41.89 & 0.20 & 0.22 & & \\
\hline HD 71974B & $4.14 \pm 0.10$ & 0.38 & 11.11 & 0.83 & 0.92 & & S99 \\
\hline HD 72760B & $8.59 \pm 0.05$ & 0.96 & 21.02 & 0.13 & 0.15 & & \\
\hline HD 77407B & $5.21 \pm 0.12$ & 1.66 & 49.77 & 0.49 & 0.48 & $\mathrm{AD}_{30}$ & M04 \\
\hline HD 91782B & $7.34 \pm 0.13$ & 1.00 & 56.11 & 0.23 & 0.20 & $\mathrm{AD}_{30}$ & \\
\hline HD 91962B & $4.19 \pm 0.13$ & 0.84 & 31.15 & 0.82 & $0.44^{\mathrm{b}}$ & $\mathrm{AD}_{30}$ & WDS \\
\hline HD 91962C & $4.10 \pm 0.16$ & 0.14 & 5.25 & 0.85 & 0.76 & $\mathrm{AD}$ & \\
\hline HD 92855B & $7.68 \pm 0.11$ & 2.93 & 105.62 & 0.18 & 0.16 & $\mathrm{AD}_{30}$ & FM30 \\
\hline HD 99565B & $3.83 \pm 0.19$ & 0.41 & 14.28 & 0.91 & 0.98 & & WDS \\
\hline GQ LeoB & $5.09 \pm 1.09$ & 0.25 & 12.40 & 0.61 & 0.99 & $\mathrm{AD}$ & \\
\hline HD 108799B & $4.31 \pm 0.09$ & 2.07 & 51.75 & 0.79 & 0.69 & $\mathrm{AD}_{30}$ & WDS \\
\hline HD 108944B & $6.34 \pm 0.10$ & 1.94 & 85.40 & 0.41 & 0.34 & $\mathrm{AD}_{30}$ & \\
\hline HD 112196B & $5.11 \pm 0.13$ & 1.50 & 51.03 & 0.59 & 0.52 & $\mathrm{AD}_{30}$ & \\
\hline HD 115043B & $8.15 \pm 0.09$ & 1.64 & 42.61 & 0.16 & 0.15 & & L05 \\
\hline HD 129333B & $6.16 \pm 0.08$ & 0.72 & 24.38 & 0.38 & 0.36 & $\mathrm{AD}_{30}$ & DM91, MH04 \\
\hline HD 134319B & $7.57 \pm 0.11$ & 5.36 & 235.66 & 0.13 & 0.13 & $\mathrm{AD}_{30}$ & L05 \\
\hline HD 135363B & $5.03 \pm 0.12$ & 0.25 & 7.28 & 0.60 & 0.84 & $\mathrm{AD}$ & L07 \\
\hline HD 139498B & $2.74 \pm 0.17$ & 0.31 & 39.50 & 1.22 & 1.00 & $\mathrm{AD}$ & WDS \\
\hline HD 142361B & $4.17 \pm 0.32$ & 0.71 & 71.21 & 0.37 & 0.22 & $\mathrm{AD}_{30}$ & G93 \\
\hline ScoPMS 27B & $3.33 \pm 0.63$ & 0.08 & 11.46 & 0.65 & 0.64 & $\mathrm{AD}$ & \\
\hline ScoPMS 52B & $3.12 \pm 0.61$ & 0.14 & 20.88 & 0.76 & 0.62 & $\mathrm{AD}$ & G93 \\
\hline PZ99 J161329.3 - 231106B & $5.47 \pm 0.60$ & 1.43 & 207.35 & 0.14 & 0.14 & $\mathrm{AD}_{30}$ & \\
\hline
\end{tabular}


Table 11

(Continued)

\begin{tabular}{|c|c|c|c|c|c|c|c|}
\hline \multirow[t]{2}{*}{ Companion } & \multirow{2}{*}{$\begin{array}{c}M_{K_{S}} \\
(\mathrm{mag}) \\
\end{array}$} & \multicolumn{2}{|c|}{$\begin{array}{l}\text { Projected } \\
\text { Separation }\end{array}$} & \multirow{2}{*}{$\begin{array}{c}M_{2} \\
\left(M_{\odot}\right) \\
\end{array}$} & \multirow[t]{2}{*}{$q$} & \multirow[t]{2}{*}{ Sample $^{\mathrm{a}}$} & \multirow[t]{2}{*}{ Ref. } \\
\hline & & $(\operatorname{arcsec})$ & $(\mathrm{AU})$ & & & & \\
\hline PZ99 J161411.0-230536B & $2.51 \pm 0.61$ & 0.22 & 32.19 & 1.17 & 0.91 & $\mathrm{AD}$ & \\
\hline HD 150554B & $6.10 \pm 0.14$ & 11.60 & 521.78 & 0.45 & 0.39 & $\mathrm{AD}_{30}$ & WDS \\
\hline HD 152555B & $6.73 \pm 0.14$ & 3.82 & 183.31 & 0.31 & 0.27 & & \\
\hline HD 155902B & $4.02 \pm 0.31$ & 0.06 & 1.74 & 0.86 & 0.90 & & \\
\hline HD 157664B & $2.84 \pm 0.16$ & 0.04 & 3.02 & 1.10 & 0.93 & & \\
\hline HD 165590B & $3.62 \pm 0.11$ & 0.45 & 16.81 & 0.90 & 0.80 & $\mathrm{AD}$ & Hip \\
\hline HD 199143B & $4.63 \pm 0.12$ & 1.05 & 50.54 & 0.49 & 0.39 & $\mathrm{AD}_{30}$ & JB01 \\
\hline HD 200746B & $5.07 \pm 0.36$ & 0.23 & 9.99 & 0.62 & 0.60 & $\mathrm{AD}$ & Hip \\
\hline HD 201989B & $7.31 \pm 0.11$ & 2.08 & 62.37 & 0.25 & 0.24 & & \\
\hline HD 203030B & $13.15 \pm 0.14$ & 11.92 & 488.84 & 0.02 & 0.02 & $\mathrm{AD}_{30}$ & MH06 \\
\hline RX J2312.0+2245B & $6.52 \pm 1.09$ & 2.86 & 429.00 & 0.38 & 0.28 & & \\
\hline RX J2313.0+2345B & $4.72 \pm 1.09$ & 1.41 & 210.90 & 0.35 & 0.31 & $\mathrm{AD}_{30}$ & \\
\hline HD 221613B & $4.48 \pm 0.12$ & 0.17 & 5.71 & 0.74 & 0.72 & & WDS \\
\hline
\end{tabular}

Notes.

a "AD" denotes companions to stars in the 128 star minimally biased AD sample (Section 9.1.1). "AD 30 " marks the 30 companions to AD stars that reside at projected separations between 0.'55 and 12 ". 5 .

b The mass ratio for the more distant companion B in the triple system HD $91962 \mathrm{ABC}$ is calculated as $q=M_{B} /\left(M_{A}+M_{C}\right)$.

References. B97: Bouvier et al. (1997); DM91: Duquennoy \& Mayor (1991); FM00: Fabricius \& Makarov (2000); G93: Ghez et al. (1993); Hip: Perryman et al. (1997, Hipparcos); JB01: Jayawardhana \& Brandeker (2001); KL98: Kohler \& Leinert (1998); L05: Lowrance et al. (2005); L07: Lafrenière et al. (2007); M04: Mugrauer et al. (2004); M92: Mermilliod et al. (1992); MH: Metchev \& Hillenbrand (2004); MH06: Metchev \& Hillenbrand (2006); P02: Patience et al. (2002); P98: Patience et al. (1998); S99: Söderhjelm (1999); WDS: Mason et al. (2001, Washington Double Star Catalog, and references therein).

(This table is also available in a machine-readable form in the online journal.)

known ages and dynamical masses will shed important light on these surprising results.

\subsection{Stellar Secondaries}

The entire survey produced 24 new stellar companions, including the three already announced in Metchev \& Hillenbrand (2004; HD 129333B, HE 373B, and RX J0329.1+0118B). HD 129333 had previously been identified as a probable long-period single-lined spectroscopic binary by Duquennoy \& Mayor (1991), and was independently resolved by König et al. (2005). Four other new binaries have since been independently confirmed in analyses of Hipparcos "problem" stars by Makarov \& Kaplan (2005; HD 26990 and HD 135363), Goldin \& Makarov (2006; HD 152555), and Goldin \& Makarov (2007; HD 155902). In addition, PZ99 J161329.3-231106, resolved by us, has since been suggested as a possible spectroscopic binary by (Guenther et al. 2007).

In addition to the 24 new systems, the physical association of 51 known binary stars was astrometrically confirmed. The star HD 91962, a previously known binary (Mason et al. 2001, and references therein), was resolved into a triple system. No higherorder multiples were resolved. A higher fraction of multiple systems might have been expected, especially given the high rate of occurrence $(34 \%-96 \%)$ of visual companions to close (spectroscopic) binary systems (Tokovinin et al. 2006). The visual companions in such multiple systems must have either fallen outside of our 12 '.5 survey radius, or been removed from our AO sample by the design of the FEPS target list, which discriminates against visual companions (see Section 7.2).

The majority (57 out of 74 ) of the binaries plus the triple system were members of the shallow survey, as a result of the requirement that no $\Delta K_{S}<4.0$ mag candidate companions were present at $>0$.'8 from deep sample stars (Section 2.1). Hence, the binaries found in the deep survey either have very low mass ratios, such that the secondary is $>4$ mag fainter than the primary at $K_{S}$, or have high mass ratios but $<0$.' 8 angular separations, so that both components were fit under the $1^{\prime \prime}$ coronagraph.

We derive $K_{S}$-band absolute magnitudes $M_{K_{S}}$ for the companions using the known distances to the primaries (Tables 1 and 2). We estimate the stellar companion masses directly from $M_{K_{S}}$ and from the primary star age using stellar evolutionary models from Baraffe et al. (1998). The mass ratios of the resolved stellar binaries ranged between 0.1 and 1.0. Including the two substellar companions, the mass ratios covered the full 0.02-1.0 range. Table 11 lists $M_{K_{S}}$ and the mass for each bona fide companion, along with projected separations and system mass ratios.

\subsection{The Apparent Proper Motion Companion to ScoPMS 214}

We devote special attention to this candidate companion here because we initially identified it as a bona fide brown dwarf companion to ScoPMS 214 in Metchev (2006).

We detected seven projected companions within 12 ".5 of ScoPMS 214 (Figure 8; Table 8). Among these, candidate companion 1 (CC1) is brightest and closest to the star, and shares the proper motion of ScoPMS 214 to within $3 \sigma$ limits over the course of 4.8 years (specifically, $\Delta \alpha / \sigma(\Delta \alpha)=0.7, \Delta \delta / \sigma(\Delta \delta)=$ $2.4, \Delta \rho / \sigma(\Delta \rho)=0.7, \Delta \theta / \sigma(\Delta \theta)=2.3)$. The apparent proper motion of $\mathrm{CC} 1$ is significantly different from the remaining three candidate companions (2, 3, and 4) to ScoPMS 214 for which we have sufficiently precise astrometric solutions. The proper motion of ScoPMS 214 is predominantly to the south

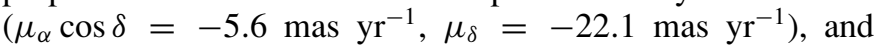
candidate companions $2-4$ systematically lag behind in their 


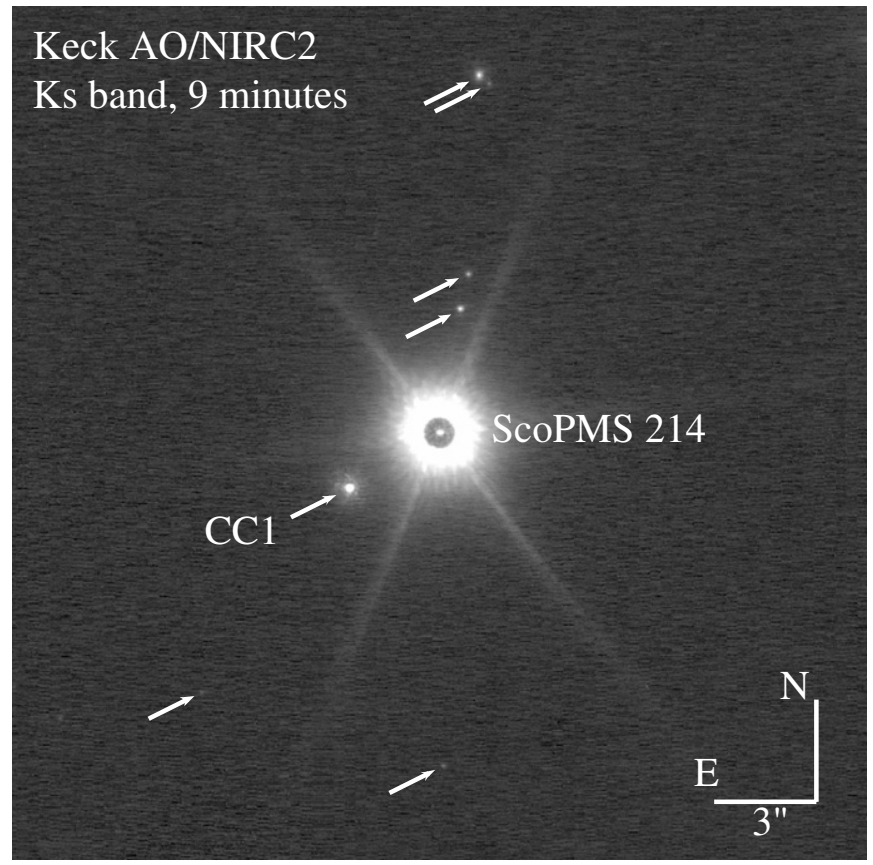

Figure 8. $K_{S}$-band image of ScoPMS 214 and its candidate companions taken with NIRC2 and the Keck AO system on 2004 June 5. The image is the median of nine $60 \mathrm{~s}$ exposures. ScoPMS 214 is occulted by a partially transmissive $1^{\prime \prime} .0$ diameter circular coronagraphic mask. The seven $\rho \leqslant 12^{\prime \prime} .5$ candidate companions listed in Table 8 are pointed out with arrows. The candidate proper motion companion $\mathrm{CC} 1$ is the brightest of the seven and closest to the star.

declination motion $\Delta \delta$, at a level of $4.5-5.3 \sigma(\Delta \delta)$ over 4.8 years. These three candidates, along with candidate companion 6 , for which the astrometry is insufficiently precise to decide its proper motion association status, are consistent with being stationary distant objects seen in projection (Figure 9). Astrometric data for candidate companions 5 and 7 does not exist over the entire 4.8 year period, and hence they are not plotted on the proper motion diagram in Figure 9. However, they are also consistent with being background stars.

In summary, $\mathrm{CC} 1$ satisfies all of the proper motion association criteria established in Section 5.2.1, whereas none of the other candidate companions to ScoPMS 214 do. Therefore, CC1 has a high likelihood of being a bound companion to ScoPMS 214 , although it could also be an unrelated member of Upper Scorpius-the parent association of ScoPMS 214-seen in projection.

It is in principle possible to distinguish between the above two possibilities in a probabilistic manner, by following a two-point correlation function analysis, as done for Upper Scorpius by Kraus \& Hillenbrand (2007). We find that the probability to find at least one chance alignment among our 23 deep sample targets that belong to young stellar associations with an unrelated $\geqslant \mathrm{M} 4$ dwarf ( $\lesssim 0.1 M_{\odot}$ ) within the same association is $\sim 2.5 \%$. That is, if $\mathrm{CC} 1$ is a member of Upper Scoprius, then there is a $97.5 \%$ probability that it is physically bound to ScoPMS 214. Similar reasoning led us to conclude in Metchev (2006) that CC1 was a bona fide companion to ScoPMS 214, which we named ScoPMS 214B.

However, as we shall see in Section 6.3.2, the spectroscopic evidence argues against membership of CC1 (ScoPMS 214“B”) in Upper Scorpius.

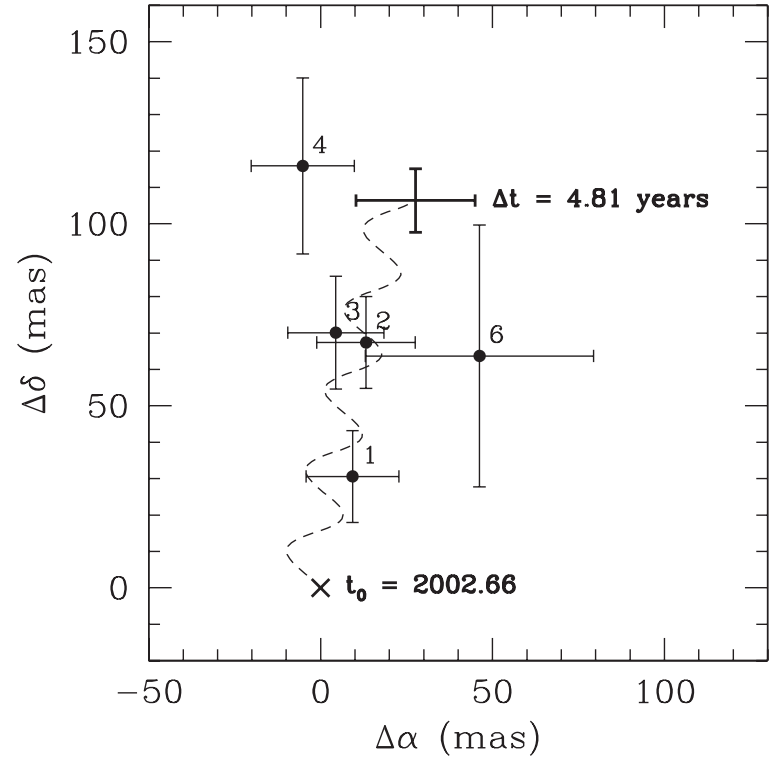

Figure 9. Proper motion diagram for candidate companions to ScoPMS 214, spanning the $\Delta t=4.81 \mathrm{yr}$ time period between the first and last epochs of observations, between 2002 August $30\left(t_{0}=2002.66 \mathrm{yr}\right)$ and 2007 June 23 . The dashed line denotes the expected relative motion of a stationary background object with respect to ScoPMS 214 between the initial epoch (marked with $\times$ ) and the final epoch (marked with thick errorbars without a solid point). The solid points with thin errorbars denote the observed changes in the relative positions of candidate companions $1,2,3,4$, and 6 . Candidate 5 was outside of the FOV of the medium $\left(20^{\prime \prime} \times 20^{\prime \prime}\right)$ NIRC2 camera during the last epoch of observations, and candidate 7 was below the detection limit during the initial epoch. Candidate 1 is ScoPMS 214"B," which shares the proper motion of ScoPMS 214 during the 4.81 year time span within $3 \sigma$ limits and is inconsistent with being a stationary background object (at the $5 \sigma$ level in declination). Candidates 2, 3, and 4 (and 7, based on observations at intermediate epochs) are all inconsistent with being proper motion companions to ScoPMS 214 and are consistent with being background objects. Candidates 5 and 6 are consistent with being either bona fide companions or unrelated background objects, that is, their status is undecided.

\subsubsection{Spectral Type and Effective Temperature of ScoPMS 214 "B"}

We obtained a $R \approx 1200 K$-band spectrum of ScoPMS 214 "B" (CC1) with Keck AO/NIRC2 on 2005 July 14. We used the 80 mas wide slit and the medium $\left(20\right.$ mas pix $\left.^{-1}\right)$ NIRC2 camera. We integrated for a total of 7.5 minutes on the companion, following an $\mathrm{ABC}$ pointing sequence with 2.5 minute integrations per pointing. We observed a nearby A0 star for telluric correction. Exposures of Ne and Ar lamps were obtained for wavelength calibration. The individual 2.5 minute exposures were pair-wise subtracted and the spectrum of ScoPMS 214"B" was traced and extracted from each exposure in a 280 mas wide ( $\approx 5.6$ PSF FWHM) aperture. The three individual spectra were median-combined and smoothed to the resolution set by the instrument configuration using a SavitskyGolay smoothing algorithm.

The resultant $K$-band spectrum of ScoPMS 214"B" is shown in Figure 10, where it is compared to Infrared Telescope Facility (IRTF)/SpeX $K$-band spectra of field $\mathbf{M}$ dwarf and $\mathrm{M}$ giant standards from the IRTF Spectral Library ${ }^{8}$ (J. T. Rayner et al. 2008, in preparation; Cushing et al. 2005), smoothed to the same resolution. The dominant atomic and molecular absorption features due to $\mathrm{Na}$ I, $\mathrm{Ca}$ I, and $\mathrm{CO}$ are identified.

The overall $K$-band continuum slope of ScoPMS 214 "B" is much closer to the continuum slopes of the $M$ dwarfs than to

\footnotetext{
8 http://irtfweb.ifa.hawaii.edu/ spex/spexlibrary/IRTFlibrary.html.
} 


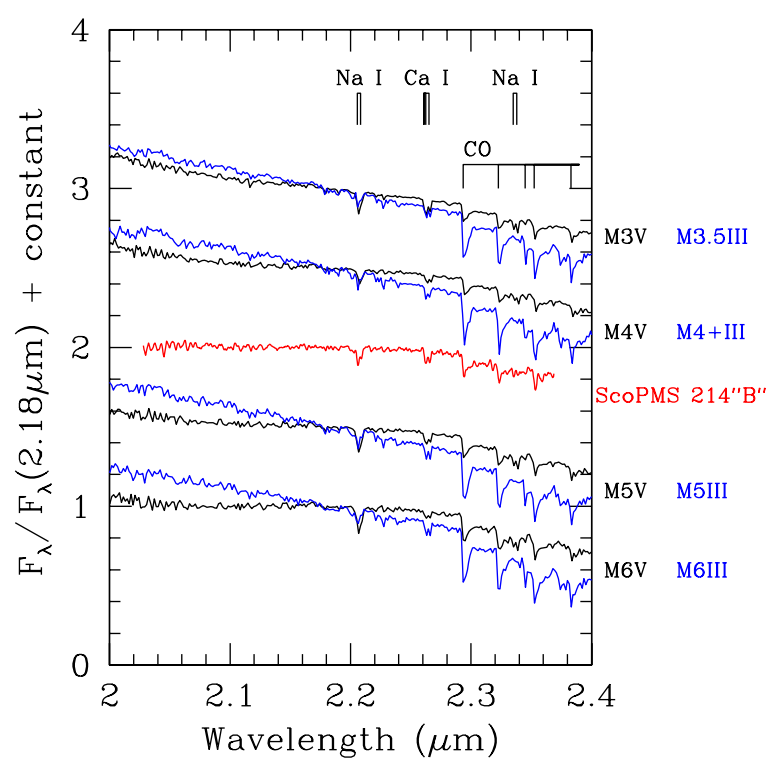

Figure 10. $K$-band spectrum (red) of ScoPMS 214"B" (CC1), compared with spectra of M3-M6 field dwarf (in black) and giants (in blue) from the IRTF Spectral Library (J. T. Rayner et al. 2008, in preparation; Cushing et al. 2005), smoothed to the same $R \approx 1200$ resolution. Dominant absorption features by $\mathrm{Na}$ I at $2.21 \mu \mathrm{m}$ (doublet) and $2.34 \mu \mathrm{m}$ (doublet), Ca I at $2.26 \mu \mathrm{m}$ (triplet), and $\mathrm{CO}$ bandheads at $\lambda \geqslant 2.29 \mu \mathrm{m}$ are identified. The comparison dwarf spectra are of Gl 388 (M3V), Gl 213 (M4V), Gl 51 (M5V), and Gl 406 (M6V), and the giants are HD 28487 (M3.5III), HD 214665 (M4+III), HD 175865 (M5III), and HD 196610 (M6III).

those of the M giants, although ScoPMS 214"B" is redder than both sets of standards. With an extinction of $A_{V} \sim 2$ mag toward Upper Scorpius, the expected reddening of ScoPMS 214 "B" at the $K$ band is negligible. Instead, the discrepancy between the continuum slopes of ScoPMS 214"B" and the $\mathrm{M}$ standards may be due to instrumental systematics between the Keck AO/NIRC2 and IRTF/SpeX spectra. In particular, accurate continuum slopes are difficult to extract from classical AO spectroscopy (Goto et al. 2003; McElwain et al. 2007) because of the chromatic behavior of the AO PSF and because of the narrow slits (here 80 mas) used to match the width of the AO PSF. Nevertheless, an independent indication that ScoPMS 214"B" has a dwarf-like surface gravity $(\log g \sim 5$ ) comes from the relatively shallow depth of the $\mathrm{CO}$ bandheads in the spectrum of ScoPMS 214"B"-comparable in strength to the $\mathrm{CO}$ bandheads of the $\mathrm{M}$ dwarfs and weaker than the $\mathrm{CO}$ bandheads of the $\mathrm{M}$ giants. This is not unusual despite the possibility that ScoPMS 214"B" may still be contracting toward the main sequence. Even at 5 Myr ages, M stars are expected to have surface gravities that are much more similar to those of dwarfs than to those of giants $(\log g \sim 1)$.

From a visual examination of the spectra in Figure 10, we estimate that ScoPMS 214"B" has an M3-M5 spectral type, based on the relative strengths of the $\mathrm{Na}$ I and $\mathrm{Ca}$ I absorption features compared to the other M dwarfs. Unfortunately, a more accurate classification based on the $K$-band spectrum alone is not possible. On one hand, the NaI $2.21 \mu \mathrm{m}$ doublet is known to be sensitive to both effective temperature and surface gravity (Gorlova et al. 2003). On the other hand, while the Ca I $2.26 \mu \mathrm{m}$ triplet is considered to be a good temperature indicator for $\mathrm{G}$ and $\mathrm{K}$ stars (Ali et al. 1995), the scatter at early- to midM spectral types is significant (Gorlova et al. 2003). This is evidenced by the nonmonotonic change in the depth of the Ca I triplet in the M3-M6 dwarf spectral-type sequence in Figure 10.
Therefore, we adopt M3-M5 as our final estimate of the spectral type of ScoPMS 214 "B."

The effective temperature corresponding to the M3-M5 range is $3250-2800 \mathrm{~K}$ (within errors of $\pm 100 \mathrm{~K}$ ), according to the field M dwarf temperature scale of Reid \& Hawley (2005, see their Table 4.1). More recent work on M dwarf effective temperatures, supported by highly accurate photometric and interferometric measurements, finds that the Reid \& Hawley (2005) scale systematically overestimates the temperatures of $<3000 \mathrm{~K}$ field M dwarfs by about $100 \mathrm{~K}$ (Casagrande et al. 2008). However, Luhman (1999) found that young M dwarfs specifically are significantly hotter than their older field counterparts. Luhman's conclusion is based on the requirement that all components of the GG Tau quadruple system lie on the same 1 Myr theoretical isochrone from the NextGen models of Baraffe et al. (1998), and is supported by population age analyses in other young associations, such as IC 348 (Luhman 1999) and the Orion Nebular Cluster Slesnick et al. 2004). Although Luhman 1999's conclusion relies on theoretical isochrones from Baraffe et al. (1998), the models in question have been shown to most successfully and, on average, fairly accurately predict the fundamental properties of pre-main-sequence stars (Hillenbrand \& White 2004). The effective temperature range of 1 Myr M3-M5 dwarfs found by Luhman (1999) is 3125-3415 K.

Such disagreement at these low effective temperatures is not unusual, given the increasing complexity of stellar spectra at $<3000 \mathrm{~K}$. The problem is even more aggravated at young ages, when the lower surface gravities of the objects further affect their photospheric appearance. Because of its specific pertinence to young M dwarfs, when considering the possibility that ScoPMS 214 "B" is a member of Upper Scorpius, we will adopt the temperature scale of Luhman (1999).

We proceed by examining two probable scenarios: (1) a "young" (5 Myr) ScoPMS 214"B" that is a member of Upper Scorpius, probably as a companion to ScoPMS 214, with $3125 \leqslant T_{\text {eff }} \leqslant 3415 \mathrm{~K}$, or (2) a "field-aged" (1-10 Gyr) ScoPMS 214 "B" that is simply seen in projection along the LOS toward ScoPMS 214 , with $2700 \mathrm{~K} \leqslant T_{\text {eff }} \leqslant 3250 \mathrm{~K}$.

\subsubsection{Is ScoPMS 214 “B” a Member of Upper Scorpius?}

To decide which of the above two scenarios is valid, and by extension, whether ScoPMS 214A and "B" form a physical pair, we compare the locations of ScoPMS 214A and "B" on an Hertzsprung-Russell (H-R) diagram with respect to the NextGen model isochrones of Baraffe et al. (1998). Mirroring the approach of Luhman (1999), we expect that if ScoPMS 214A and "B" were bound and hence coeval, they should lie on the same isochrone. Since the temperature of ScoPMS 214 "B" is $\sim 3000 \mathrm{~K}$ regardless of the scenario considered, the use of the dust-free NextGen models is justified. Indeed, the more recent DUSTY models from the Lyon group (Chabrier et al. 2000) do not extend above $3000 \mathrm{~K}$, since dust is not expected to form in stellar photospheres at such high effective temperatures.

The H-R diagram analysis is illustrated in Figure 11. In the "young" ScoPMS 214"B" scenario we have adopted the mean distance to Upper Scorpius members, $145 \pm 40 \mathrm{pc}$, for both ScoPMS 214A and "B." The bolometric luminosity of ScoPMS 214 "B" is then $\log L / L_{\odot}=-2.37 \pm 0.24$, where we have used bolometric corrections for M3-M5 dwarfs from Tinney et al. (1993) and Leggett et al. (1996). In this scenario, ScoPMS 214 "B" lies on the 1 Gyr isochrone (i.e., on the main sequence), in disagreement with the positioning of ScoPMS 214A above 


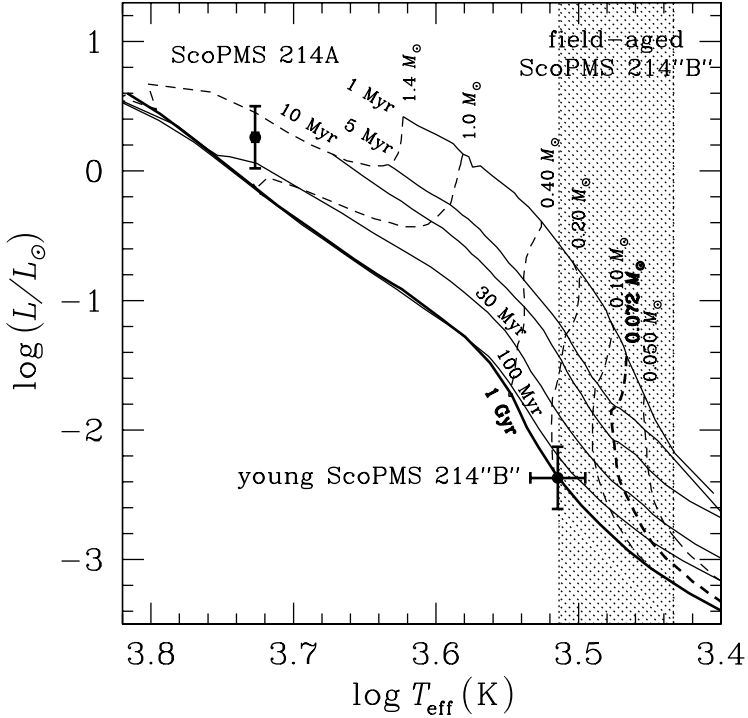

Figure 11. H-R diagram of the candidate binary ScoPMS 214A"B" with evolutionary models for $0.05-1.4 M_{\odot}$ objects from Baraffe et al. (1998). The continuous lines are isochrones and the dashed lines are evolutionary tracks at constant mass. The thick (1 Gyr) isochrone approximates the main sequence, and the thick evolutionary track corresponds to the minimum hydrogen-burning mass. The positions of ScoPMS 214A and "B" under the assumption of equidistance and membership in Upper Scorpius (the "young" ScoPMS 214"B" scenario; Section 6.3.2) are shown with solid points with errorbars. The shaded region represents the range of effective temperature allowed for ScoPMS 214" $\mathrm{B}$ " if it were an unassociated field-aged (1-10 Gyr) M dwarf. Since in the "young" scenario the candidate binary components do not lie on the same theoretical isochrone, ScoPMS 214"B" is probably not a member of Upper Scorpius. Instead, it is most likely a foreground field $\mathrm{M}$ dwarf.

the main sequence. Presuming that ScoPMS 214A is itself not an unresolved binary, the discrepancy indicates that the assumed distance range for ScoPMS 214" B" is incorrect and that probably it is not a member of the Upper Scorpius association. While ScoPMS 214A also lies slightly beneath an extrapolation of the $5 \mathrm{Myr}$ isochrone, its position is not inconsistent with the adopted age for Upper Scorpius, especially given the physical extent ( $\sim 40 \mathrm{pc}$ core radius) of the association.

In the "field-aged" ScoPMS 214"B" case, the object is not a member of Upper Scorpius, and hence its heliocentric distance and bolometric luminosity are not constrained. This case is presented by the shaded region in Figure 11. Given the range of luminosities at which the shaded region intersects the main sequence, the distance to ScoPMS 214"B" is between 70 and $145 \mathrm{pc}$.

Therefore, we conclude that ScoPMS 214"B" is probably not a member of Upper Scorpius and hence probably not a physical companion to ScoPMS 214. Instead, it is likely to be a foreground $\mathrm{M}$ dwarf seen in projection against Upper Scorpius. We arrive at this conclusion despite the apparent agreement between the proper motions of ScoPMS 214 and ScoPMS 214 "B" over nearly 5 years. The reason for the apparent agreement is probably due to the relatively small proper motion of ScoPMS 214 (23 mas $\mathrm{yr}^{-1}$ ), which is near the precision limits of our astrometric calibration even over a 5 year period. Ongoing astrometric monitoring of this system and measurements of the individual radial velocities of the two components will allow us to discern the difference in their space motions.

\section{SURVEY INCOMPLETENESS AND SAMPLE BIASES}

Before addressing the frequency of wide substellar companions in our sample (Section 8), we present a brief summary of the factors that affect the completeness of our survey (Section 7.1) and discuss the various sample biases (Section 7.2). A detailed completeness analysis based on these considerations is presented in the Appendix.

\subsection{Survey Incompleteness}

The principal factors that influenced the completeness of our deep survey can be divided into three categories: (1) geometrical, defined by the inner and outer working angles (IWA and OWA, respectively) of the survey ( 0.55 and 12 '.5, respectively) and by the distribution of heliocentric distances of the sample targets; (2) observational, defined by the flux limits of the survey relative to the predicted brightness of substellar companions; and (3) orbital, defined by the fraction of the orbital phase space observed. For the sake of simplicity in estimating the total survey incompleteness, we have assumed that the distributions of the orbital semimajor axis and mass for substellar companions are $d N / d \log a \propto a^{0}$ and $d N / d M_{2} \propto M_{2}^{0}$, respectively. Other common distributions for these parameters are explored in Appendix A.4, and are found not to affect the final completeness estimate by more than a factor of 1.24 .

We find that the combined completeness of the deep survey to substellar companions in 28-1590 AU semimajor axes ranges from $64.8 \%$ at the $0.072 M_{\odot}$ hydrogen-burning mass limit to $47.0 \%$ at the $0.012 M_{\odot}$ deuterium-burning mass limit. The deep survey is severely incomplete $(<30 \%$ completeness) to companions below $0.012 M_{\odot}$ and maximally complete $(64.9 \%)$ at and above $0.090 M_{\odot}$. Over the combined $0.012-0.072 M_{\odot}$ brown dwarf mass range, we estimate that the deep survey is complete to $62 \%$ of substellar companions with orbital semimajor axes between $28 \mathrm{AU}$ and $1590 \mathrm{AU}$ (Appendices A.3.3-A.5). We combine this estimate with the observational results to obtain the underlying substellar companion frequency in Section 8.1.

\subsection{Sample Biases}

Our survey sample carries an important bias against visual binaries, inherited from the FEPS target selection policy. The FEPS sample excluded certain types of visual binaries to minimize photometric confusion in Spitzer's 1".5-30" beam at 3.6-70 $\mu \mathrm{m}$ wavelengths (Meyer et al. 2006). In particular,

1. all FEPS sample stars were required to have no projected companions closer than 5" in 2MASS, and

2. stars older than $100 \mathrm{Myr}$ were also required to have no projected 2MASS companions closer than $15^{\prime \prime}$, unless the companions were both bluer in $J-K_{S}$ and fainter at $K_{S}$ by $>3$ mag. ${ }^{9}$

The above two criteria create a nontrivial bias against stellarmass companions in our AO sample. Because of the seeinglimited dynamic range of 2MASS $\left(\sim 4.5 \mathrm{mag}\right.$ at $5^{\prime \prime}, \sim 2.5 \mathrm{mag}$ at $3^{\prime \prime}$; see Figure 11 in Cutri et al. 2003), criterion 1 excludes near-equal magnitude (i.e., near-equal mass) stellar companions. Criterion 2 then further excludes fainter, red (and, hence, lowermass) companions, although only around the $>100 \mathrm{Myr}$ old stars.

Therefore, any analysis of the stellar multiplicity in our survey would tend to underestimate the true stellar companion rate. In particular, if we adopt the median distance for the complete $\mathrm{AO}$ sample (Table 3) and the orbital period distribution for solar

\footnotetext{
9 For reasons of generating a statistically significant sample size, $<100 \mathrm{Myr}$ old stars were allowed to violate this criterion in FEPS
} 
mass binaries from Duquennoy \& Mayor (1991), we find that the above FEPS selection criteria have probably excluded $\sim 25 \%$ of stellar binaries, mostly near-equal mass systems. We do not address this bias further. In Section 9.1, we only note that it has a systematic effect on our estimate of the CMF, in the sense that we have underestimated the relative frequency of near-equal mass binaries.

An additional bias against binary stars, relevant only to the deep portion of our AO survey, is incurred by our on-the-fly selection against $\Delta K_{S}<4$ mag projected companions at 0 '.8$13^{\prime \prime}$.0 from our deep-sample coronagraphic targets (criterion 2 in Section 2.1). However, by keeping track of which stars were delegated to the shallow sample in this manner, we have accounted for this bias in our analysis of the CMF in Section 9.1.

Finally, our AO sample also carries a slight bias against substellar secondaries because of the second FEPS selection criterion above. This bias affects only 100-500 Myr old targets in the deep sample with well-separated $\left(\geqslant 5^{\prime \prime}\right)$ massive brown dwarf secondaries. Fortunately, because of the shallow depth of 2MASS $\left(K_{S} \lesssim 15 \mathrm{mag}\right)$ and its limited dynamic range $\left(\Delta K_{S} \lesssim 6 \mathrm{mag}\right)$ within our 12 ".5 AO survey radius, the effect of this bias is negligible. Based on the range of semimajor axis distributions assumed for substellar companions considered in Appendix A.4, we find that this criterion would have excluded $\leqslant 0.5 \%$ of detectable $\geqslant 60 M_{\text {Jup }}$ substellar companions. Over the entire substellar companion mass sensitivity range of our survey $\left(13-75 M_{\text {Jup }}\right)$ the effect of this bias is negligible $(<0.1 \%)$. We will, therefore, ignore it in the rest of the discussion.

\section{THE FREQUENCY OF WIDE SUBSTELLAR COMPANIONS}

Throughout the remainder of this paper, we will use the general terms "substellar companion" and "brown dwarf companion" to refer to a $0.012-0.072 M_{\odot}\left(13-75 M_{\text {Jup }}\right)$ brown dwarf secondary in a $28-1590$ AU orbit around a young Sun-like star, unless otherwise noted.

\subsection{Results from the Present Survey}

Having discovered two bona fide brown dwarf companions among the 100 stars in the deep sample, we estimate the true substellar companion fraction that these detections represent. We do so by following a Bayesian approach to derive confidence ranges for the implied frequency of detectable substellar companions, and by applying the incompleteness correction derived in Appendix A.5.

Strictly speaking, the probability of obtaining $x$ successful outcomes (e.g., brown dwarf companion detections) from a number of repetitions of an experiment with a binary outcome is governed by a binomial distribution. In practice, the large number of experiments (100) and the small number of successful outcomes in our case $(x=2)$ mean that the probability of detecting $x$ brown dwarfs given an expected mean rate $\mu$ is well approximated by a Poisson probability distribution:

$$
P(x \mid \mu)=\frac{\mu^{x} e^{-\mu}}{x !} .
$$

We are interested in finding what is the probability distribution for the actual mean rate $\mu$ given $x$ detections, i.e., we seek the probability density function (pdf) $P(\mu \mid x)$.

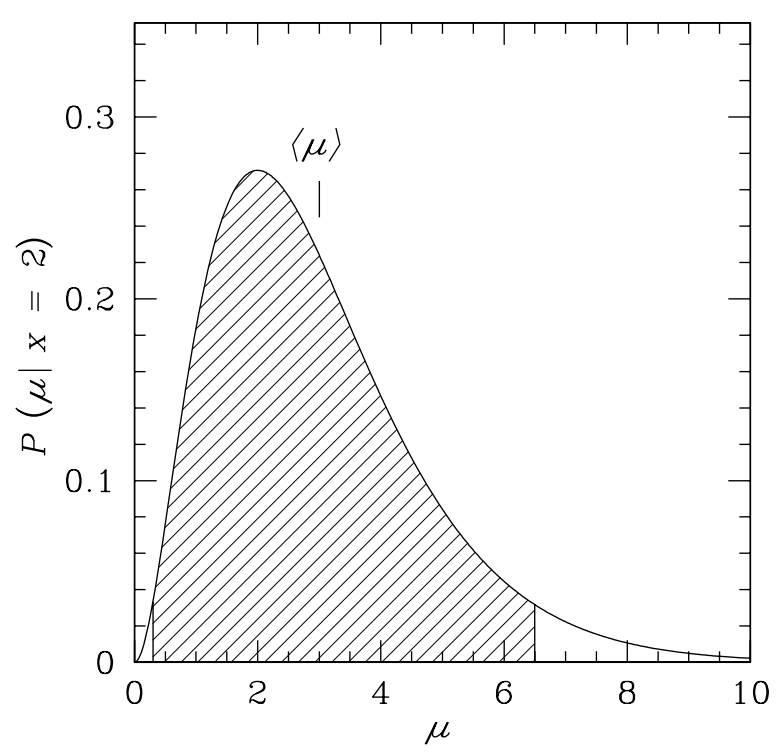

Figure 12. Probability density distribution $P(\mu \mid x=2)$ for the expected substellar companion detection rate in our survey per 100 stars, given $x=2$ detections. The curve is a Gamma distribution (Equation (5)), with a peak at $\mu=\mu_{\mathrm{ML}}=2$, but a mean value of $\langle\mu\rangle=x+1=3$. The minimal $2 \sigma(95.4 \%)$ confidence interval on $\langle\mu\rangle, 0.3<\langle\mu\rangle<6.5$, is indicated by the shaded region under the curve.

The result follows from Bayes' Theorem (Rainwater \& Wu 1947; Papoulis 1984):

$$
P(\mu \mid x)=\frac{P(x \mid \mu) P(\mu)}{P(x)},
$$

where the $P$ s denote "probability distributions" rather than identical functional forms. $P(\mu)$ is the "prior" and summarizes our expectation of the state of nature prior to the observations. $P(x \mid \mu)$ is the "likelihood" that $x$ positive outcomes are observed given a mean of $\mu . P(\mu \mid x)$, the distribution of interest, is the "posterior" probability that the state of nature is $\mu$, given $x$ positive outcomes. $P(x)$ is a normalization factor equal to the sum of all probable outcomes $P(x \mid \mu)$, given the distribution of the prior $P(\mu)$ :

$$
P(x)=\int_{0}^{\infty} P(x \mid \mu) P(\mu) d \mu .
$$

We assume no previous knowledge of the state of nature, and adopt a prior that minimizes the introduction of subjective information, imposing only a condition of nonnegativity: $P(\mu)=1$ for $\mu \geqslant 0, P(\mu)=0$ for $\mu<0$. That is, we assume that all positive substellar companion detection rates are equally probable. Inserting Equation (2) into Equations (3) and (4), we obtain

$$
P(\mu \mid x)=P(x \mid \mu)=\frac{\mu^{x} e^{-\mu}}{x !} .
$$

That is, the pdf of $\mu$ is a Gamma distribution that peaks at the observed detection rate $x$ (Figure 12). Due to the asymmetry of the Gamma distribution, the mean value $\langle\mu\rangle$ is higher than the most likely value $\mu_{\mathrm{ML}}:\langle\mu\rangle=x+1=3>\mu_{\mathrm{ML}}=2$.

We determine the confidence interval $\left[\mu_{l}, \mu_{h}\right]$ of the frequency of substellar companions $\mu$ at a desired confidence level CL by integrating $P(\mu \mid x)$ between $\mu_{l}$ and $\mu_{h}$. We set the lower and upper bounds $\mu_{l}$ and $\mu_{u}$ of the confidence interval CL so 
that (see Figure 12)

$$
\int_{\mu_{l}}^{\mu_{u}} P\left(\mu^{\prime} \mid x\right) d \mu^{\prime}=\mathrm{CL}
$$

and

$$
P\left(\mu_{l} \mid x\right)=P\left(\mu_{u} \mid x\right) .
$$

Equations (6) and (7) define the minimum size confidence interval $\left[\mu_{l}, \mu_{u}\right]$ for confidence level CL (Kraft et al. 1991). The system of equations cannot be analytically inverted, and has to be numerically solved for $\mu_{l}$ and $\mu_{u}$. We do so for the equivalent to the 1,2 , and 3 Gaussian sigma $(68.2 \%$, $95.4 \%$, and $99.7 \%$, respectively) confidence intervals. The respective confidence ranges for $\mu$ are $0.9-3.9,0.3-6.5$, and 0.07-9.9 detectable brown dwarf companions for a survey of 100 stars.

Having thus addressed the statistical uncertainties associated with the small number of companion detections, we now apply the estimated survey completeness correction (62\%) to $\mu_{\mathrm{ML}}$ and to the confidence interval limits of $\mu$. We find that the most likely rate of occurrence of brown dwarf companions in 28-1590 AU orbits around 3-500 Myr old F5-K5 stars is $\mu_{\mathrm{ML}}=2 \% / 0.62=3.2 \%$. The confidence intervals on this estimate are $1.5 \%-6.3 \%$ at the $1 \sigma$ level, $0.5 \%-10.5 \%$ at the $2 \sigma$ level, and $0.1 \%-16.0 \%$ at the $3 \sigma$ level, and are not a strong function of the prior (Kraft et al. 1991). The mean frequency, $3 \% / 0.62=4.8 \%$, is higher than the most likely value, but the exact value of the mean frequency is dependent on the Bayesian prior. The higher mean frequency of wide brown dwarf companions (6.8\%) that we reported in Metchev (2006) was due to the inclusion of ScoPMS 214"B" as a substellar companion. We have now shown that ScoPMS 214"B" is most probably an unrelated foreground star seen in projection along the LOS toward ScoPMS 214 (Section 6.3.2).

The above results for the frequency of substellar companions are built upon simple assumptions for the semimajor axis and mass distributions of substellar secondaries (Section 7.1; for greater detail, see Appendix A.2). However, our conclusions do not strongly depend on these assumptions. As we show in Appendix A.4, when either or both distributions are varied within empirically reasonable limits, the substellar companion frequency remains unchanged to within a factor of 1.24. If, as we argue in Section 9.2, the orbital period distribution of substellar companions is the same as for stellar companions, our frequency estimate is accurate to within a factor of 1.06 .

\subsection{Comparison with Previous Companion Searches}

\subsubsection{Radial Velocity Surveys}

Precision radial velocity surveys for extrasolar planets have revealed that brown dwarf secondaries are unusually rare $(<0.5 \%)$ in $0-3 \mathrm{AU}$ orbits from $\mathrm{G}$ and $\mathrm{K}$ stars - a phenomenon termed "the brown dwarf desert" (Marcy \& Butler 2000). The dearth of brown dwarfs in radial velocity surveys is evident with respect to the observed 0-3 AU frequencies of both extrasolar planets (5\%-15\%; Marcy \& Butler 2000; Fischer et al. 2002) and stellar secondaries (11\%; Duquennoy \& Mayor 1991) around Sun-like stars. That is, brown dwarfs are $\approx 20$ times rarer than planets and stellar companions in 0-3 AU orbits.

We found that $3.2_{-2.7}^{+7.3} \%$ ( $2 \sigma$ confidence interval) of young Sun-like stars have $0.012-0.072 M_{\odot}$ companions with semimajor axes between 28 and $1590 \mathrm{AU}$ (Section 8.1). The much wider orbits probed in the present survey prevent a direct parallel with the radial velocity results. Nevertheless, at face value the evidence indicates that the frequency of wide brown dwarf companions to Sun-like stars is, on average, a factor of $\sim 3$ smaller than that of $0-3$ AU extrasolar planets, and a factor of $\sim 6$ greater than the frequency of 0-3 AU brown dwarfs. The difference with the exoplanet frequency is not statistically significant. The frequencies of 28-1590 AU and 0-3 AU brown dwarfs differ at the $98.6 \%$ significance level. That is, wide brown dwarf companions to Sun-like stars are roughly comparable in frequency to radial velocity extrasolar planets, and are probably more common than radial velocity brown dwarfs.

\subsubsection{Wide Stellar Companions}

Based on the Duquennoy \& Mayor (1991) orbital period distribution and multiplicity of Sun-like stars, the frequency of $28-1590 \mathrm{AU}$ stellar companions is $\approx 24 \%$. Our estimated frequency of brown dwarfs is a factor of $\sim 8$ smaller, and significantly (at the $1-10^{-8}$ level) so. Therefore, brown dwarf secondaries are indeed less common than stellar secondaries in the 28-1590 AU orbital range.

\subsubsection{Other Direct Imaging Surveys for Substellar Companions}

A large number of direct imaging surveys have been completed to date, covering a wide range in primary mass and in sensitivity to substellar companions. Despite the disparate characteristics of these surveys, there are now enough data to analyze the ensemble of the results.

We compare our AO survey to all previously published direct imaging surveys for substellar companions to $\geqslant 0.2 M_{\odot}$ primaries. We include only surveys targeting $\geqslant 15$ stars that also contain at least a cursory reference to the parent sample statistics and to the substellar companion discovery rates. All such surveys, to our present knowledge, are listed in Table 12. Additional direct imaging surveys certainly exist. However, any published results from these have tended to report only individual detections. We have also added the radial velocity results of Marcy \& Butler (2000) to this list of direct imaging surveys for comparison. For each published survey, Table 12 lists the number, median spectral type, age, primary mass, and heliocentric distance of the sample stars. For most surveys, these values have been inferred from the description or listing of the sample in the referenced publication. For some surveys, however, these parameters have been inferred based on the stated focus of the survey (e.g., Sun-like stars) or, where appropriate, based on the properties of stars in the solar neighborhood. Table 12 also lists the maximum probed projected separation, the sensitivity to companion mass, the number of detected brown dwarf companions, and the rate of detection of brown dwarf companions.

Although an incompleteness analysis is crucial for the correct interpretation of survey results, the majority of published results do not contain such analysis. Therefore, any comparison among surveys has to be based solely on the mean or median survey sample statistics and sensitivities. Taking the ensemble statistics of all direct imaging surveys for substellar companions at face value, without accounting for their varying degrees of incompleteness, we find that the mean detection rate is 1.0 substellar companions per 100 stars. Given the very low number of detections per survey (0-2), the results from all imaging companion surveys are fully consistent with each other.

We have plotted the substellar companion detection rates of all surveys on a primary mass versus outer probed separation diagram in Figure 13. The outer probed separation is defined 
Table 12

Direct Imaging Surveys for Brown Dwarf Companions

\begin{tabular}{|c|c|c|c|c|c|c|c|c|c|c|c|c|}
\hline Survey & Sample $^{\mathrm{a}}$ & Stars & $\mathrm{SpT}^{\mathrm{b}}$ & $\begin{array}{l}\text { Age }^{\mathrm{b}} \\
(\mathrm{Gyr})\end{array}$ & $\begin{array}{c}\text { Mass }^{\mathrm{b}} \\
M_{\odot} \\
\end{array}$ & $\begin{array}{c}d^{\mathrm{b}} \\
(\mathrm{pc})\end{array}$ & $\begin{array}{l}\rho_{\text {out }}{ }^{b} \\
(\mathrm{AU})\end{array}$ & $\begin{array}{c}\text { Sensitivity }^{\mathrm{b}} \\
\left(M_{\mathrm{Jup}}\right)\end{array}$ & $N_{\mathrm{BD}}$ & $\begin{array}{l}f_{\mathrm{BD}} \\
(\%) \\
\end{array}$ & Label & Comments \\
\hline Marcy \& Butler (2000) & Field & 500 & G5 ? & 5.0 & $1.0 ?$ & $\ldots$ & 3 & 0.5 & 2 & 0.40 & MB00 & 1 \\
\hline Schroeder et al. (2000) & Field & 23 & M1.5 & 5.0 & 0.5 & 3.5 & 53 & 30 & 0 & 0 & S00 & \\
\hline Brandner et al. (2000) & Cha T, Sco-Cen & 24 & M1.5 & 0.005 & 0.6 & 150 & 1500 & 3 & 0 & 0 & B00 & \\
\hline Oppenheimer et al. (2001) & Field & 164 & M1 & 5.0 & 0.5 & 5.9 & 177 & 35 & 1 & 0.61 & O01 & \\
\hline Gizis et al. (2001) & Field & $60 ?$ & $\mathrm{~K}$ ? & 5.0 & $0.8 ?$ & $<25$ & 10000 & 40 & 3 & 5.00 & G01 & 2 \\
\hline Potter et al. (2002) & Young field & 31 & G5 ? & 0.5 & 1.1 & $20 ?$ & $200 ?$ & 30 & 1 & 3.22 & $\mathrm{P} 02$ & 3 \\
\hline Hinz et al. (2002) & Field & 66 & M3.5 & 5 & 0.2 & 5.8 & 1480 & 40 & 0 & 0 & H02 & \\
\hline Neuhäuser \& Guenther (2004) & Tuc-Hor & 25 & G5 ? & 0.035 & 1.2 & 60 & 4320 & 13 & 1 & 4.00 & NG04 & 4 \\
\hline McCarthy \& Zuckerman (2004) & Young field & 83 & M1 & 0.3 & 0.5 & 15 & 225 & 30 & 0 & 0 & MZ04 & 5 \\
\hline Masciadri et al. (2005) & Young field & 28 & M0 & 0.012 & 0.8 & 21 & 147 & 5 & 0 & 0 & M05 & \\
\hline Carson et al. (2005) & Field & 80 & $\mathrm{~K} 7$ & 5 & 0.7 & 10.3 & 155 & 50 & 0 & 0 & CE05 & \\
\hline Luhman et al. (2005) & IC 348 & 150 & M4.5 & 0.002 & 0.2 & 315 & 1600 & 6 & 0 & 0 & LMG05 & \\
\hline Lowrance et al. (2005) & Young field & 45 & K5 & 0.15 & 0.75 & 30 & 200 & 10 & 1 & 2.22 & L05 & \\
\hline Chauvin et al. (2005b) & Young field & 50 & $\mathrm{~K}$ ? & $0.035 ?$ & $1.0 ?$ & $60 ?$ & $420 ?$ & $5 ?$ & 1 & 2.00 & CL05 & 6 \\
\hline Kouwenhoven et al. $(2005,2007)$ & Sco OB2 & 199 & A & 15 & $2.0 ?$ & 130 & 520 & 30 & 1 & 0.50 & K05 & 7 \\
\hline Luhman et al. (2007b) & Young field & 73 & G6 & 0.12 & 1.1 & 30 & 4500 & $13 ?$ & 1 & 1.37 & $\mathrm{~L}_{07}$ & 8 \\
\hline Luhman et al. (2007b) & Field & 48 & G3 & 5.0 & 1.0 & 22 & 3300 & $30 ?$ & 1 & 2.08 & $\mathrm{LO}_{2}$ & 8 \\
\hline Tanner et al. (2007) & Taurus & 15 & K7 & 0.002 & 1.5 & 140 & 140 & 50 & 0 & 0.00 & T07 & 9 \\
\hline Biller et al. (2007) & Young field & 54 & $\mathrm{~K} 2$ & 0.03 & 1.0 & 25 & 50 & 5 & 0 & 0 & B07 & \\
\hline Lafrenière et al. (2007) & Young field & 85 & K0 & 0.1 & 1.0 & 22 & 200 & 2 & 1 & 1.18 & LD07 & \\
\hline Lafrenière et al. (2008) & IC 348 & 126 & M2.5 & 0.002 & 0.29 & 160 & 960 & 13 & 0 & 0 & L08 & \\
\hline Kraus et al. (2008) & Upper Sco & 82 & M0 & 0.005 & 0.7 & 145 & 435 & 13 & 1 & 1.22 & K08 & 10 \\
\hline This work & Young field & 100 & G5 & 0.08 & 1.1 & 115 & 1440 & 13 & 2 & 1.98 & $\mathrm{MH}$ & \\
\hline
\end{tabular}

Notes.

a The target source sample for each work.

b Median value for the primary stars in the survey.

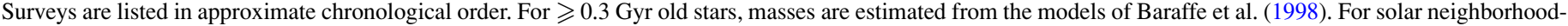

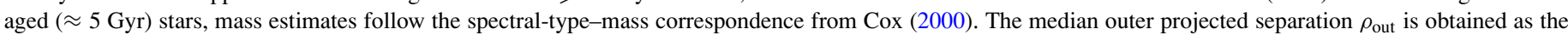

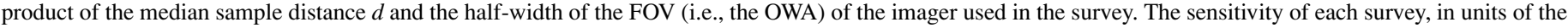

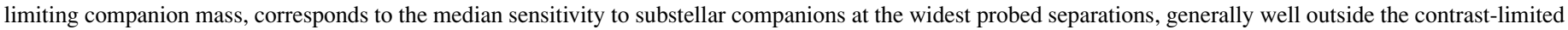

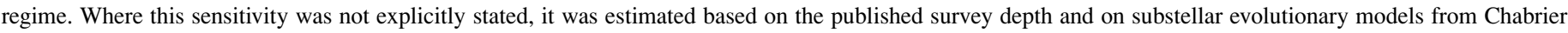

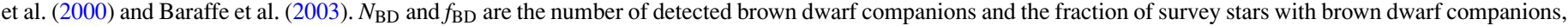

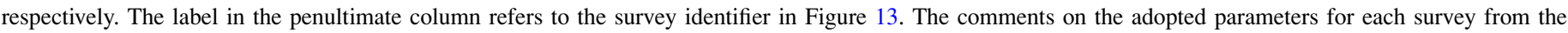

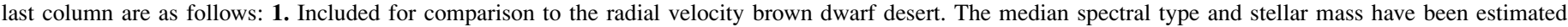

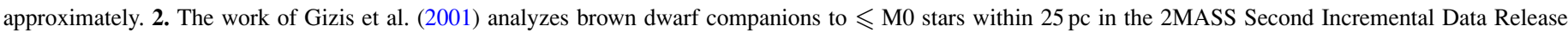

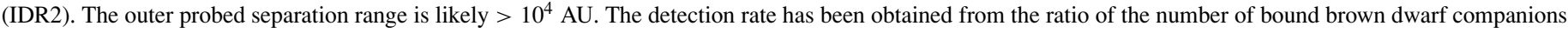

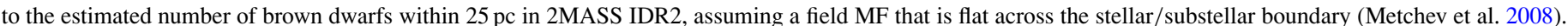

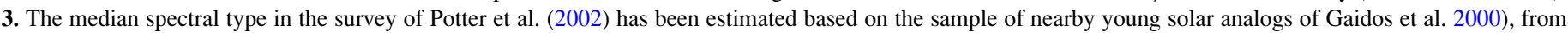

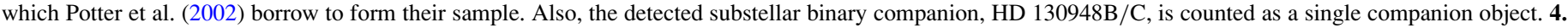

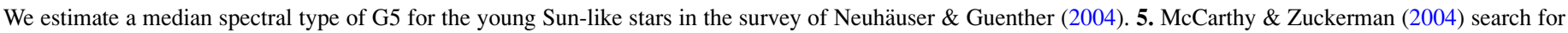

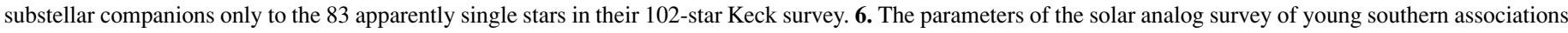

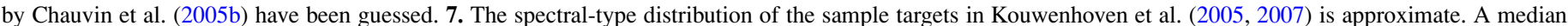

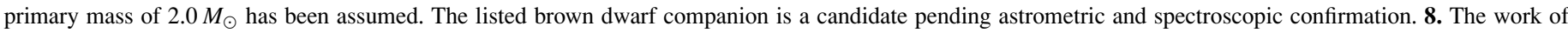

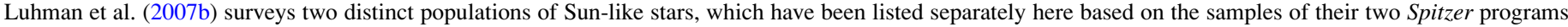

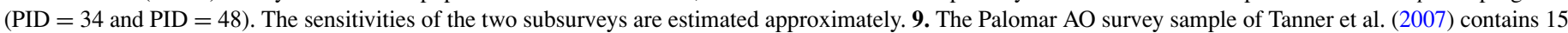

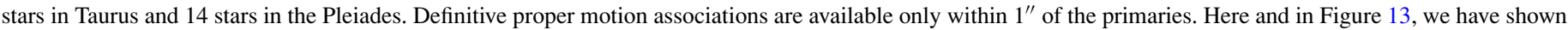

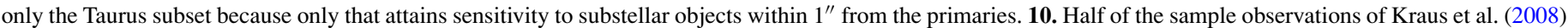

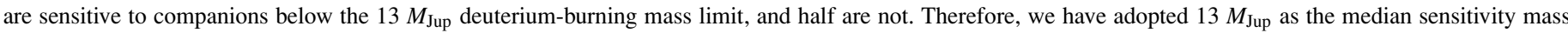
limit of the survey. The listed brown dwarf companion is a candidate pending astrometric and spectroscopic confirmation.

simply as the product of the survey angular radius (generally, the half-width of the imaging detector) and the median heliocentric distance for the survey sample. The diagram reveals that the surveys with the highest detection rates of substellar companions reside in a distinct locus in the upper right quadrant of the diagram, delimited by the dotted line. All surveys outside of this region have detection rates $\leqslant 0.6 \%$, whereas all surveys within the region have generally higher $(0.5 \%-5.0 \%)$ detection rates. This fact transcends survey sensitivity considerations. Some of the most sensitive companion surveys with the smallest likely degrees of incompleteness, such as the radial velocity survey of Marcy \& Butler (2000) and the simultaneous differential imaging (SDI) surveys of Masciadri et al. (2005) and Biller et al. (2007), lie outside of the dotted region and have detection rates well below $1 \%$. That is, unless all of these highly sensitive surveys did not detect brown dwarf companions through some improbable happenstance, a significant population of brown dwarf companions apparently exists at $\gtrsim 150$ AU separations from $\gtrsim 0.7 M_{\odot}$ stars. Brown dwarf companions appear to be less frequent both at smaller orbital separations from Sun-like stars and at wide separations from lower-mass stars. The dearth of brown dwarf companions to $0.2-0.6 M_{\odot}$ stars is likely due to a combination of the lower multiplicity rate of low-mass stars and the tendency of low-mass binaries to predominantly 


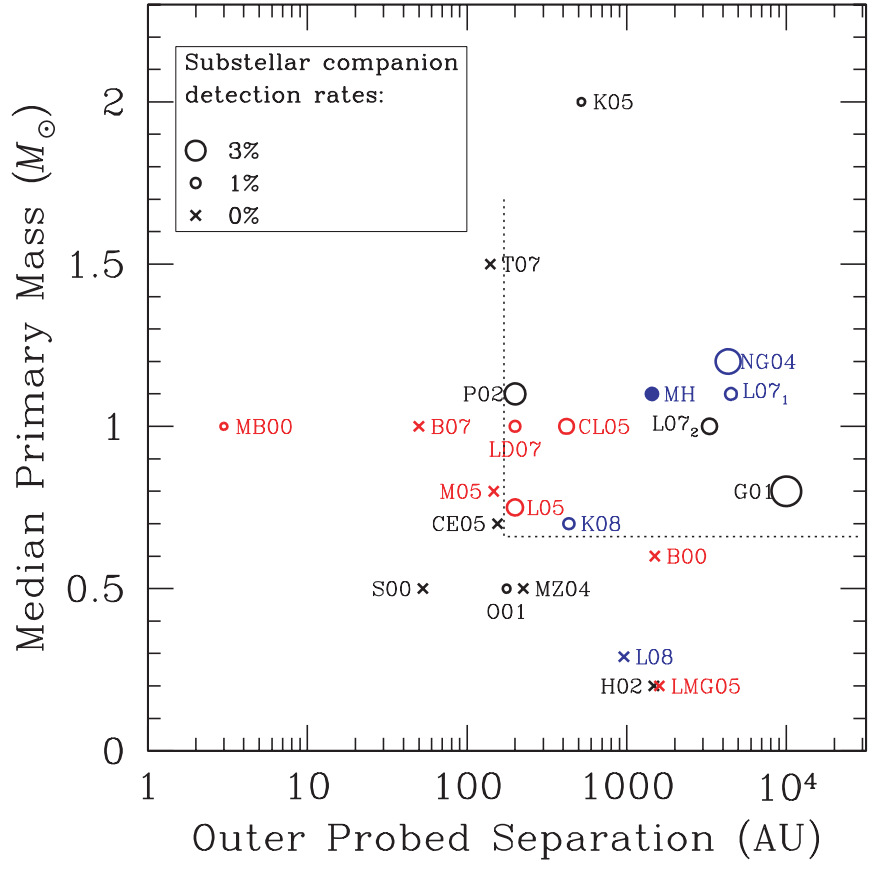

Figure 13. Substellar companion detection rates of the published direct imaging surveys listed in Table 12. Circular symbols denote surveys with at least one detection; crosses denote surveys with no detections. The filled circle denotes the present work. The size of the circular symbols is proportional to the survey detection rate prior to corrections for survey incompleteness. Black symbols denote the least sensitive surveys, with $\geqslant 30 M_{\text {Jup }}$ median companion mass sensitivity in the background-limited regime. Blue symbols denote surveys with median companion sensitivities between 13 and $30 M_{\text {Jup }}$. Red symbols mark surveys with the highest sensitivity, $<13 M_{\text {Jup }}$. The survey labels are as listed in the penultimate column of Table 12. The locus delimited by a dotted line contains only surveys with nonzero detections, with detection rates ranging from 0.5 to $5 \%$. All surveys outside of this region have detection rates $\leqslant 0.6 \%$.

exist in close-in near-equal mass systems (e.g., Burgasser et al. 2007; Allen 2007, and references therein). The surveys with the highest detection rates are those targeting very wide companions to $\sim 1 M_{\odot}$ stars.

It is important to reiterate again that none of the detection rates for any of the surveys in Figure 13 have been corrected for systematic or incompleteness effects. In particular, there is a strong bias against the detection of substellar companions in narrow orbits in all direct imaging surveys because of contrast limitations. In addition, the position of each survey along the abscissa is based on the median outer probed separation, whereas most companions are detected at smaller projected separations. Therefore, the increase in the frequency of substellar companions to $\gtrsim 0.7 M_{\odot}$ stars probably begins well within $150 \mathrm{AU}$. In Section 9.2, we argue that the peak of the brown dwarf companion projected separation distribution may in fact occur at $\sim 25 \mathrm{AU}$, as would be expected from the Duquennoy \& Mayor (1991) binary orbital period distribution.

\section{DISCUSSION}

\subsection{The Substellar and Stellar CMF}

The salient characteristic of the present imaging survey is its high sensitivity to low-mass $\left(M_{2} \leqslant 0.1 M_{\odot}\right)$ companions to solar analogs, that is, to systems with mass ratios $q \lesssim 0.1$. We found only seven such companions among 74 binary and one triple systems: the two brown dwarfs HD 49197B and HD 203030B, and the 0.08-0.14 $M_{\odot}$ stars HD 9472B, HE 373B, RX
J0329.1+0118B, HD 31950B, and PZ99 J161329.3-231106B (Table 11). A naïve expectation from the mass function (MF) of isolated objects (Kroupa 2001; Chabrier 2001) would require approximately as many $<0.1 M_{\odot}$ companions as there are $>0.1 M_{\odot}$ companions. Therefore, it appears that there is a dearth of widely separated both substellar and low-mass stellar companions to Sun-like stars.

To assess the reality and magnitude of this effect, we need a uniform survey of a well-characterized sample of binaries. Unfortunately, our full survey sample is not adequate for such an analysis because the imaging depths of the deep and the shallow subsurveys are vastly different, and because the sample is subjected to the combined effect of three different biases against binary stars (Section 7.2). We could, in principle, focus only on the deep survey of 100 young stars, for which we have a well-characterized completeness estimate. However, doing so would not avoid any of the binarity biases. Furthermore, the deep survey sample contains only 19 of all 75 binaries and triples, only six of which are in the $0.55-12^{\prime \prime} .5$ angular separation range, for which we estimated incompleteness (Section 7.1). This number is too low for a meaningful analysis of the CMF.

Nevertheless, we can undo some of the binarity biases and recover certain rejected stellar secondary companions by revisiting how binaries were removed from the deep sample during survey observations. We discuss this procedure and reconstruct a sample that is minimally biased against binaries in the following.

\subsubsection{Defining a Minimally-Biased Sample}

We construct a less biased, larger sample of stars by adding to our 100 star deep sample all other $\leqslant 500$ Myr old stars that were initially selected to be in the deep sample but for which no coronagraphic exposures were taken because of the discovery of a close-in $\left(00^{\prime \prime} 8-13^{\prime \prime} .0\right) \Delta K_{S}<4$ mag companion (see Section 2.1). Since we did not inherit this bias from the larger FEPS program sample, but rather imposed the criteria ourselves, we knew the parent sample and were able to undo the bias exactly. The resulting "augmented" deep (AD) sample is minimally biased against binaries to the extent to which we control the sample generation.

There are 28 binaries excluded in this manner that contribute to a total of 128 young stars in the AD sample. Among these are a total of 46 binaries and one triple, of which 30 systems (including the triple) have companions between 0'.55 and 12'.5 from the primary. Members of the AD sample are distinguished in the last column of Table 11, where the 30 members with 0'.55-12".5 companions are marked with " $\mathrm{AD}_{30}$."

We assume that the young binaries added from the shallow sample do not have additional fainter tertiary companions between 0.55 and 12 .'.5 that would have been detectable had we exposed them to the depth of the longer coronagraphic images. Given the $\approx 10 \%$ ratio of double-to-triple systems in the study of Duquennoy \& Mayor (1991), and the fact that the 28-1590 AU orbital range $\left(\approx 10^{4.7}-10^{7.3}\right.$ days at $1 M_{\odot}$ total mass $)$ includes approximately $42 \%$ of all companions $\left(0-10^{10}\right.$ day periods) probed in Duquennoy \& Mayor (1991), we would expect that $\approx 0.10 \times 0.42 \approx 4 \%$ of systems have a tertiary component in a $28-1590$ AU orbit. In comparison, the $1: 46 \approx 2 \%$ ratio of triples to binaries in our subsample indicates that such an assumption is not unreasonable - on average, we may have missed one lowmass (possibly substellar) tertiary component. Therefore, we have potentially suffered only a small loss in completeness by 
including stars for which we do not have deep coronagraphic exposures.

The $\mathrm{AD}$ and the $\mathrm{AD}_{30}$ samples remain biased against binaries, although mostly against near-equal mass systems (Section 7.2). Because we have placed an upper age limit of $500 \mathrm{Myr}$ for membership in these samples and because of the logarithmically uniform distribution of stellar ages in the parent FEPS sample, the bias against lower-mass stellar secondaries (FEPS binarity criterion 2; Section 7.2) affects less than a quarter of the stars in the AD sample: only those that are 100-500 Myr old.

The detectability of the $\mathrm{AD}_{30}$ secondaries within the greater $\mathrm{AD}$ sample is subject to the same set of target selection criteria and to the same geometrical, observational, and orbital incompleteness factors (see Appendix A.3) as for the deep survey. Therefore, we can estimate the completeness of the $\mathrm{AD}$ sample to the detection of secondaries in $28-1590 \mathrm{AU}$ semimajor axes in the same manner as done for the deep survey.

The completeness to $0.012-0.072 M_{\odot}$ substellar companions in $28-1590 \mathrm{AU}$ semimajor axes in the deep survey ranges from $47.0 \%$ to $64.8 \%$, depending on companion mass (see Appendix A.3). For masses $\geqslant 0.090 M_{\odot}$ the deep survey is maximally complete at $64.9 \%$. The integrated completeness to $0.01-1.0 M_{\odot}$ companions is $\approx 64 \%$ (compare with the $62 \%$ integrated completeness to $0.012-0.072 M_{\odot}$ substellar companions; Section 7.1). Given the $300.55-12.5$ binaries in the $\mathrm{AD}_{30}$ sample, we would expect a total of $30 / 0.64 \approx 47 \pm 9$ binaries with $28-1590$ AU semimajor axes in the $128 \mathrm{star}$ AD sample, where the error on that estimate is propagated as $\sqrt{30} / 0.64$ (by pure coincidence, this is exactly how many multiple systems (47) are present in the AD sample). The incompleteness-corrected frequency of $0.01-1.0 M_{\odot}$ companions in $28-1590 \mathrm{AU}$ orbits in the AD sample is thus $47 / 128=37 \% \pm 7 \%$. This is somewhat higher than the $24 \%$ integrated over the corresponding $10^{4.7}$ $10^{7.3}$ day orbital period from Duquennoy \& Mayor (1991). Despite the bias against binaries, the higher multiplicity fraction of stars in our survey is not unexpected because of our superior sensitivity to very low-mass companions and our focus on young stars, which tend to more often be found in multiples (e.g., Ghez et al. 1993, 1997).

\subsubsection{The Distribution of Companion Masses}

In their $\mathrm{G}$ dwarf multiplicity study, Duquennoy \& Mayor (1991) found that the MFs of isolated field stars and of 0.1-1.0 $M_{\odot}$ stellar companions to solar-mass primaries were indistinguishable. We now revisit this conclusion in light of our more sensitive imaging data and in the context of more recent determinations of the field MF.

The mass ratio distribution for our selection of 30 young binaries in the $\mathrm{AD}_{30}$ sample is shown in Figure 14. The distribution is well fitted by a power law of the form

$$
\log \left(\frac{d N}{d \log q}\right)=\delta \log q+b,
$$

equivalent to $d N / d \log q \propto q^{\delta}$ or $d N / d q \propto q^{\delta-1} \equiv q^{\beta}$. The best-fit value for the power-law index is $\delta=0.61$ or, equivalently, $\beta=-0.39 \approx-0.4$. The reduced $\chi^{2}$ of the fit is adequate, 1.5 , and given only 3 degrees of freedom (dof), a higher-order functional fit is not warranted. The $\chi^{2}$ contours of $\beta$ and $b$ indicate that the parameters are correlated. By integrating over all possible values for $b$, we find that the $68 \%$ (one Gaussian $\sigma$ ) and $95 \%$ confidence intervals for $\beta$ are $-0.75<\beta<-0.03$ and $-0.93<\beta<0.14$, respectively.
We compare this mass ratio distribution to the known MF of isolated field objects from Chabrier (2003). Because the masses of the primary stars in our sample are distributed closely around $1 M_{\odot}$ (Figure $1(\mathrm{~b})$ ), we can directly compare the distribution of the (unitless) mass ratios with the field MF (in units of $M_{\odot}$ ). That is, the mass ratio distribution of our sample is essentially equivalent to the CMF in units of $M_{\odot}$ since $q=M_{2} / M_{1} \approx M_{2} / M_{\odot}$. The power-law index $\beta$ of the CMF is analogous to the linear slope $\alpha$ (Salpeter value -2.35 ) of the field MF. As is evident from Figure 14, the CMF of our sample of young binaries is very different (reduced $\chi^{2}=7.6$ ) from the MF of field objects.

A potentially more sensitive comparison between the observed mass-ratio distribution and any model MFs (e.g., lognormal, power law) could be obtained using a KolmogorovSmirnov (K-S) test. We do not perform Monte Carlo simulations to degrade the MF models to match the observed data, as would be necessary for a statistically rigorous application of the $\mathrm{K}-\mathrm{S}$ test, but instead compare the models to the incompletenesscorrected data. Although the $\mathrm{K}-\mathrm{S}$ test is not strictly applicable with such an approach, the results from the test are nevertheless illustrative. Thus, a one-sample $\mathrm{K}-\mathrm{S}$ test finds only a $2 \times 10^{-8}$ probability that the observed CMF originates from the $\log$-normal field MF of Chabrier (2003). The K-S probability that the fitted power law in Equation (8) with $\beta=-0.39$ is the correct parent CMF is 7\%. Ostensibly, the best agreement (58\% K-S probability) with the incompleteness-corrected data is reached by a log-normal mass ratio distribution with a mean and standard deviation of 0.39. A similar log-normal CMF was independently inferred by Kraus et al. (2008) in their analysis of resolved binaries in Upper Scorpius. However, we note that in our case, the difference between the probabilities of the power-law and log-normal parent CMFs (7\% versus 58\%) is not statistically significant in the context of the $\mathrm{K}-\mathrm{S}$ test. Therefore, given the already adequate reduced $\chi^{2}$ of the power-law fit from Equation (8), we disregard the potentially better, but statistically less well-motivated, agreement between the data and the higher-order log-normal parameterization (three free parameters), in favor of the lower-order (two free parameters) power law.

A value near zero for our CMF power-law exponent $\beta$ is consistent with the $\mathrm{MF}$ of $<0.1 M_{\odot}$ objects in the field $(-1.0<\alpha \lesssim 0.6$; Chabrier 2001; Kroupa 2002; Allen et al. 2005) and in young stellar associations $(-1 \lesssim \alpha \lesssim 0$; Hillenbrand \& Carpenter 2000; Slesnick et al. 2004; Luhman 2004). However, the monotonic rise of the CMF throughout the entire 0.01-1.0 $M_{\odot}$ mass range and the lack of a turnover near $0.1 M_{\odot}$ disagree with MF determinations for stars in the 0.1$1.0 M_{\odot}$ interval, where $\alpha$ ranges between -0.5 and -2 (Kroupa 2002). That is, in the stellar mass regime, the CMF and the MF of isolated stars are distinctly different.

We should note that the results from our companion survey may not be ideally suited for determining the CMF of both brown dwarf and stellar companions. Indeed, we recall that our AD sample is biased against various types of visual binaries (Section 7.2). However, as we discussed in Section 9.1.1, the bias against binarity in the AD sample is mostly against nearequal mass systems that occupy the highest mass ratio bin in Figure 14. That is, the power-law index $\beta$ of the CMF would only further increase in value if the bias against near equalmass binaries in our survey sample were taken into account, and the CMF would become even more disparate from the field MF. 

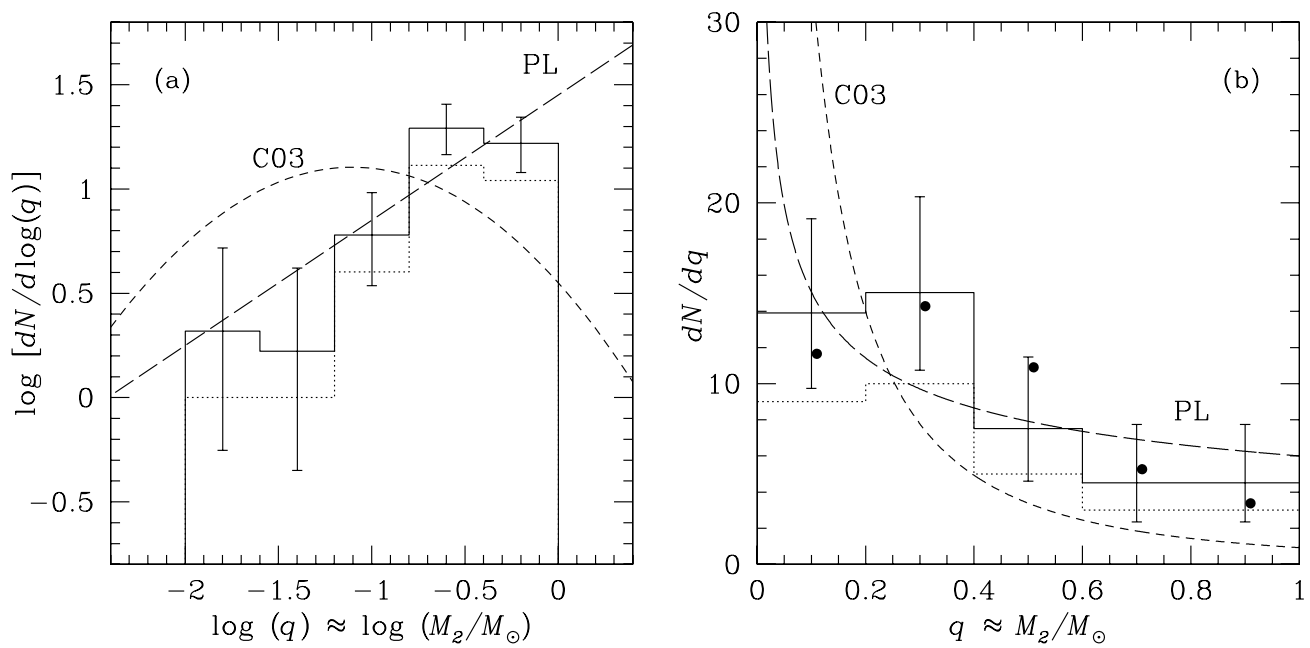

Figure 14. Mass ratio distribution for the $30 \leqslant 0.5 \mathrm{Gyr}$ old binaries in our $\mathrm{AD}_{30}$ sample (see Section 9.1) in terms of $\log (d N / d \log q)$ (a) and $d N / d q$ (b). The dotted histogram traces the observed data, while the solid histogram delineates the incompleteness-corrected data. Further incompleteness due to bias against near-equal binary systems exists in the highest mass ratio bin, but has not been taken into account in the present incompleteness correction. The long-dashed line is a power-law (PL) fit to the data, $d N / d \log q \propto q^{\beta+1}$, with an index of $\beta=-0.39 \pm 0.36(1 \sigma$ limit $)$. The short-dashed line represents the log-normal MF of field objects from Chabrier (2003, C03) in units of $M_{\odot}$, normalized to the incompleteness-corrected data. We note that because the primary masses for stars in our sample are $\approx 1 M_{\odot}$, then $q=M_{2} / M_{1} \approx M_{2} / M_{\odot}$. The log-normal field MF peaks at $\mu=0.08 M_{\odot}$ and has a width of $\sigma=0.69$ (in logarithmic mass units). The Salpeter index in these units is $\alpha=-2.35$. The solid points in panel (b) are the incompleteness-corrected data from Duquennoy \& Mayor (1991), normalized to our data. The Duquennoy \& Mayor (1991) data have been offset slightly to the right from ours for clarity.

Our conclusion counters the established view that the MF of $0.1-1.0 M_{\odot}$ binary components is indistinguishable from the MF of isolated objects. In arriving at the original result, Duquennoy \& Mayor (1991) had compared the $0.1<q \leqslant 1.0$ binary mass ratio distribution of their sample stars with an earlier form of the field MF from Kroupa et al. (1990). Since the mass ratio distribution of $q>0.1$ binaries in our sample is consistent with that of Duquennoy \& Mayor (see Figure 14(b)), the difference in the results stems from our superior sensitivity to $q \leqslant 0.1$ binaries and from the recently improved knowledge of the MF of low-mass $\left(<0.2 M_{\odot}\right)$ stars in the field.

Similar conclusions were independently reached by Shatsky \& Tokovinin (2002) and by Kouwenhoven et al. (2005) in their direct imaging studies of the visual multiplicity of intermediate mass (2-20 $\left.M_{\odot}\right)$ B and A stars, respectively. These two surveys found that the mass ratio distribution of 45-900 AU intermediate mass binaries follows an $f(q) \propto q^{\beta}$ power law, where $\beta$ is -0.5 (Shatsky \& Tokovinin 2002) or -0.33 (Kouwenhoven et al. 2005). Our determination that $\beta=-0.39 \pm 0.36$ for companions to solar-mass stars indicates that the shape of the CMF found by Shatsky \& Tokovinin (2002) and Kouwenhoven et al. (2005) is not specific to intermediate mass stars. Considered together, these three sets of results provide a strong evidence of a significant difference between the MFs of wide secondaries and of isolated field objects. That is, the mass ratio distribution of 28-1590 AU binaries is inconsistent with random pairing of stars drawn from the initial mass function (IMF) over a vast range of primary and companion masses. We discuss the implications of this conclusion on shaping the dearth of brown dwarf secondaries to stars below.

\subsection{The Brown Dwarf Desert as a Result of Binary Star Formation}

The inferred 0.01-1.0 $M_{\odot}$ CMF (Section 9.1.2) naturally explains the scarcity of wide brown dwarf companions without the need to invoke formation or evolutionary scenarios specific to substellar companions. The functional form of the widebinary CMF is also consistent with results for tighter binaries from radial velocity studies. Thus, Mazeh et al. (2003) found that the CMF of $\mathrm{K}$-dwarf binaries in $0-4 \mathrm{AU}$ orbits is also a rising function of mass over the $0.07-0.7 M_{\odot}$ range. Their data are consistent with a power-law index of $\beta \approx-0.4$, in full agreement with the $-0.3 \leqslant \beta \leqslant-0.5$ values for $28-1590 \mathrm{AU}$ binaries found in Shatsky \& Tokovinin (2002), Kouwenhoven et al. (2005, 2007), and this paper.

It perhaps may be argued that, given the disparate sensitivity systematics and statistical treatments in these diverse samples, such an overall agreement is merely coincidental. Indeed, differences in the mass ratio distributions of short- versus long-period binaries within single uniform samples have been previously suggested, with dividing periods of 1000 days ( 2 AU; Duquennoy \& Mayor 1990) or 50 days ( $\sim 0.3 \mathrm{AU}$; Halbwachs et al. 2003). However, subsequent analyses by Duquennoy \& Mayor (1991) and Mazeh et al. (2003) showed that the evidence for such discontinuities was inconclusive because of relatively small number statistics. At the same time, the combined set of direct imaging and spectroscopic data referenced here points to an approximately uniform functional form for the CMF over 1.5 orders of magnitude in primary mass $\left(0.6-20 M_{\odot}\right), 3.3$ orders of magnitude in companion mass $(0.01-$ $\left.20 M_{\odot}\right)$, and 4.7 orders of magnitude in physical separation (0.03-1590 AU). That is, we see strong evidence of a universally uniform shape of the CMF.

Given such universality, it is interesting to consider whether the CMF can explain the very low frequency of brown dwarfs found not only from direct imaging, but also from radial velocity surveys. Because stellar and substellar companions to Sun-like stars appear to be derived from the same CMF (Section 9.1.2), we can presume that the Duquennoy \& Mayor (1991) period distribution of $\geqslant 0.1 M_{\odot}$ stellar secondaries also holds for substellar companions. Based on this period distribution, the frequency of secondary companions in $0-3 \mathrm{AU}$ orbits is $11 \%$. Brown dwarfs account for $\leqslant 0.5 \% / 11 \%=4.5 \%$ of these. For comparison, brown dwarfs account for $\sim 3.2 \% / 24 \%=$ 
$13 \%$ of all secondaries in $28-1590$ AU orbits (here $24 \%$ is the overall frequency of stars with 28-1590 AU companions, based on an integration of the Duquennoy \& Mayor (1991) period distribution). In the context of our inferred power-law CMF, the value of the index $\beta$ would need to be as high as 0.2 to reproduce the $\sim 3$ times smaller relative frequency of radial velocity brown dwarfs. This does not well agree with our $95 \%$ confidence limits on $\beta(-0.93<\beta<0.14$; Section 9.1.2). However, we also noted that our value for $\beta$ is systematically underestimated because of the bias against near-equal mass binaries in our AO sample.

It is, therefore, conceivable that the radial velocity brown dwarf desert around $\mathrm{G}$ stars represents just the low-mass, narrow-orbit end of a CMF that spans 3.3 dex in secondary mass and 4.7 dex in the orbital semimajor axis. The problem that would then need to be addressed is not why brown dwarf companions specifically are so rare, but why the CMF differs so significantly from the MF of isolated substellar and stellar objects over all orbital ranges.

In such a universal CMF scenario, brown dwarfs would be expected to peak in frequency at semimajor axes determined by the binary period distribution: at $\approx 31$ AU from solar mass stars or at projected separations of $\approx 31 / 1.26=25 \mathrm{AU}$ (see Appendix A.2 for explanation of the factor of 1.26). At first glance, this is not consistent with the diagram of survey detection rates in Figure 13, where we found that (prior to correction for survey incompleteness) the highest detection rates occurred in surveys probing projected separations $\gtrsim 150$ AU. However, we pointed out that Figure 13 compares only the median outer projected separations probed by the various surveys, whereas most companions tend to be discovered at smaller projected separations (Section 8.2.3). In addition, we need to consider that projected separations of $25 \mathrm{AU}$ are usually well within the contrast-limited regime of existing direct imaging surveys of young nearby $(50-100 \mathrm{pc})$ stars. Our own survey is less than $40 \%$ complete to objects at the hydrogen-burning mass limit in $31 \mathrm{AU}$ semimajor axis orbits (see Appendix A.3). That is, a number of $\sim 30$ AU brown dwarfs around solar-mass stars may have simply been missed in direct imaging surveys because of insufficient imaging contrast.

Unfortunately, neither of the two most sensitive direct imaging surveys that probe well within $150 \mathrm{AU}$ (Masciadri et al. 2005; Biller et al. 2007) detect any substellar companions. However, their sample sizes are not large (54 and 28 , respectively), and the null detection rates do not place significant constraints on our hypothesis. Conversely, the recent discovery of several probable radial velocity brown dwarfs in $>3 \mathrm{AU}$ orbits by Patel et al. (2007) lends support to the idea that brown dwarfs are more common at wider separations, as would be inferred by extrapolation from the Duquennoy \& Mayor (1991) results to substellar companions.

Finally, the $\approx 0.012 M_{\odot}\left(\approx 13 M_{\text {Jup }}\right)$ deuterium-burning mass, above which we limit our analysis, does not necessarily mark the bottom of the MF of isolated objects. Based on results from three-dimensional smoothed particle hydrodynamic simulations, Bate et al. (2002) and Bate \& Bonnell (2005) estimated that the opacity limit for gravo-turbulent fragmentation may be as low as 3-10 $M_{\text {Jup }}$. Adopting $3 M_{\text {Jup }}$ as the limit and extrapolating the inferred CMF to $<13 M_{\mathrm{Jup}}$ masses, we find that subdeuterium-burning "planetary-mass" companions, if able to form through gravo-turbulent fragmentation, exist in $\geqslant 30 \mathrm{AU}$ orbits around only $\lesssim 1 \%$ of Sun-like stars.

\section{CONCLUSION}

We have presented the complete results from a direct imaging survey for substellar and stellar companions to 266 Sun-like stars performed with the Palomar and Keck AO systems. We discovered two brown dwarf companions in a subsample of 100 3-500 Myr old stars imaged in deep coronagraphic observations. Both were already published in Metchev \& Hillenbrand (2004, 2006). In addition, we discovered 24 new stellar companions to the stars in the broader sample, five of which are in very low-mass ratio $q \sim 0.1$ systems.

Following a detailed consideration of the completeness of our survey, we found that the frequency of $0.012-0.072 M_{\odot}$ brown dwarf companions in 28-1590 AU orbits around 3-500 Myr old Sun-like stars is $3.2_{-2.7}^{+3.1} \%$ ( $2 \sigma$ limits). This frequency is marginally higher than the frequency of 0-3 AU radial velocity brown dwarfs, and is significantly lower than the frequency of stellar companions in 28-1590 AU orbits. The frequency of wide substellar companions is consistent with the frequency of extrasolar giant planets in 0-3 AU orbits. A comparison with other direct imaging surveys shows that substellar companions are most commonly detected in surveys probing out to at least $150 \mathrm{AU}$ projected separations from $\gtrsim 0.7 M_{\odot}$ stars. However, because of bias against the direct imaging of faint close-in companions, brown dwarf secondaries are likely also common at smaller projected separations.

Considering the two detected brown dwarf companions as an integral part of the broader spectrum of stellar and substellar companions found in our survey, we infer that the mass ratio distribution of 28-1590 AU binaries and, hence, the MF of 28-1590 AU secondary companions to solar-mass primaries, follows a $d N / d M_{2} \propto M_{2}^{\beta}$ power law, with $\beta=-0.39 \pm 0.36$ ( $1 \sigma$ limits). This distribution differs significantly from the MF of isolated objects in the field and in young stellar associations, and is inconsistent with random pairing of individual stars with masses drawn from the IMF. In this context, the observed deficiency of substellar relative to stellar companions at wide separations arises as a natural consequence of the shape of the $\mathrm{CMF}$, and does not require explanation through formation or evolutionary scenarios specific to the substellar regime.

Comparing our CMF analysis with results from other direct imaging and radial velocity surveys for stellar and substellar companions, we find tentative evidence for universal behavior of the CMF across the entire 0-1590 AU orbital semimajor axis and the entire $0.01-20 M_{\odot}$ companion mass range. Such a universal CMF is not inconsistent with the marked dearth of brown dwarfs in the radial velocity brown dwarf desert around Sun-like stars. That is, the properties of brown dwarf companions at any orbital separation are conceivably an extension of the properties of stellar secondaries. Hence, we predict that the peak in semimajor axes of brown dwarf companions to solar-mass stars occurs at $\approx 30 \mathrm{AU}$. Extrapolating the inferred $\mathrm{CMF}$ to masses below the deuterium-burning limit, we find that if $0.003-0.012 M_{\odot}$ "planetary-mass" secondaries can form through gravo-turbulent fragmentation, they should exist in $\geqslant 30 \mathrm{AU}$ orbits only around less than $1 \%$ of Sun-like stars.

We thank R. Dekany, M. Troy, and M. Britton for sharing with us their expertise on the Palomar AO system, R. Burress and J. Hickey for assistance with PHARO, R. Campbell, P. Amico, and D. Le Mignant for their guidance with using Keck AO, K. Matthews and D. Thompson for help with NIRC2, and our telescope operators at the Palomar Hale and Keck II telescopes. We are also grateful to $\mathrm{K}$. Matthews for loaning us a pinhole 
mask for the astrometric calibration of PHARO, and to both R. Dekany and $\mathrm{K}$. Matthews for key insights into the design of the calibration experiment. Use of the FEPS Team database has proven invaluable throughout the course of our survey. We thank J. Carpenter for building and maintaining the database. For the target selection, age-dating, and determination of distances to the FEPS sample stars, we acknowledge the tremendous amount of work performed by E. Mamajek. This publication makes use of data products from the 2MASS, funded by the NASA and the NSF. The authors also wish to extend special thanks to those of Hawaiian ancestry on whose sacred mountain of Mauna Kea we are privileged to be guests. Support for S.A.M. was provided by NASA through the Spitzer Legacy Program under contract 1407 and through the Spitzer Fellowship Program under award 1273192. Research for this paper was also supported by the NASA/Origins R\&A program.

Facilities: Keck II Telescope, Palomar Observatory's 5 m Telescope

Note Added in Proof: After this manuscript went to press, the authors became aware of the following two recent developments:

1. Andersen et al. (2008) published an analysis of the IMF for an ensemble of seven young clusters, from which they found strong evidence that, rather than being flat across the substellar/stellar boundary, the IMF in fact turns over at substellar masses $\left(d N / d M \propto M^{-a}, \alpha<0\right)$. Their result agrees with the updated log-normal shape of the IMF hypothesized by Chabrier (2005; $\left.\mu=0.25 M_{\odot}, \sigma=0.55\right)$. The Andersen et al. (2008) and the Chabrier (2005) results bring the IMF of isolated objects in closer agreement with our inferred $\mathrm{CMF}$ that rises as a function of companion mass across the substellar/stellar boundary. Nevertheless, if we take into account the estimated $\sim 25 \%$ incompleteness of our CMF to near-equal mass companions (Section 7.2), the CMF for companions to solar-mass stars and the Chabrier (2005) IMF remain inconsistent at the $99.97 \%$ probability level according to the $\mathrm{K}-\mathrm{S}$ test.

2. Zuckerman \& Song (2008) published a parallel analysis of the CMF based on an overview of the substellar companion direct imaging literature. They found that the CMF of young substellar companions is best fit by a $d N / d M \propto M^{-1.2 \pm 0.2}$ power law. This appears inconsistent with our finding: $d N / d M \propto M^{0.39 \pm 0.36}$. However, the two results can be reconciled when we consider how different the primary mass ranges in the two analyses are. The mass range of our primary stars is relatively narrow, $0.7-1.3 M_{\odot}$, and hence our CMF closely mirrors the binary mass ratio distribution. Zuckerman \& Song's literature sample encompasses a much broader primary mass range, $0.1-3 M_{\odot}$, a significant fraction of which are below $0.5 M_{\odot}$. Given the now known inverse proportionality relation of the binary mass ratio distribution, and the fact that the mass function of primary stars rises toward lower masses, the relative overrepresentation of lower mass substellar companions in Zuckerman \& Song's sample is not surprising. Indeed, these two empirical studies can be viewed as independent manifestations of the recent analytical predictions by Kouwenhoven et al. (2008) who infer observable outcomes of various pairing algorithms for binary stars. In the framework of Kouwenhoven et al. (2008), both our and Zuckerman \& Song's result exclude the possibility of random pairing as a mechanism for forming binary systems, a conclusion that we reached based on our data alone in Section 9.1.2. At the same time, both results closely resemble the predicted outcomes for the split-core pairing (SPC) binary formation scenario investigated by Kouwenhoven et al., in which the total binary mass is drawn from a system IMF (e.g., Kroupa 2001) and then the mass ratio of the binary is drawn from a pre-defined mass ratio distribution.

\section{APPENDIX}

\section{INCOMPLETENESS OF THE DEEP SURVEY}

Here we examine the factors affecting the sensitivity of the deep survey to substellar companions (Appendix A.1), and, based on several assumptions about the semimajor axis and mass distributions of wide substellar companions (Appendix A.2), we estimate the completeness of the survey (Appendix A.3). We find that variations in the parameters of the semimajor axis and mass distributions have little effect (Appendix A.4) on the final completeness estimate. This final estimate (Appendix A.5) is used in Section 8 in combination with the observational results from our survey to obtain the actual frequency of substellar companions.

\section{A.1. Factors Affecting Survey Completeness}

Several factors need to be taken into account when estimating the detectability of substellar companions to our stars. These include (1) possible sample bias against stars harboring substellar secondaries, (2) choice of substellar cooling models, (3) observational constraints (i.e., survey radius, imaging contrast, and depth), and (4) physical parameters of the stellar/substellar systems (flux ratio, age, heliocentric distance, orbit).

As discussed in Section 7.2, the deep sample is largely unbiased toward substellar companions, that is, factor (1) can be ignored. For the basis substellar cooling models (2), we rely on the DUSTY and COND models of the Lyon group (Chabrier et al. 2000; Baraffe et al. 2003). These have been used, either alone or in parallel with the models of the Arizona group (Burrows et al. 1997), in all other studies of substellar multiplicity. Our choice, therefore, ensures that our results are comparable with the existing work on the subject. The remaining factors (3 and 4) motivate the rest of the discussion here.

\section{A.2. Assumptions}

We base our incompleteness analysis on three assumptions: (1) that the distribution of the semimajor axes $a$ of substellar companions to stars is flat per unit logarithmic interval of semimajor axis, $d N / d \log a \propto a^{0}$ (or equivalently, $d N / d a \propto$ $a^{-1}$ ) between $10 \mathrm{AU}$ and $2500 \mathrm{AU}$, (2) that this implies a logarithmically flat distribution in projected separations $\rho$ : $d N / d \log \rho \propto \rho^{0}$ (i.e., $d N / d \rho \propto \rho^{-1}$ ), and (3) that the MF of substellar companions is flat per linear mass interval $\left(d N / d M_{2} \propto M_{2}^{\beta}=M_{2}^{0}\right)$ between $0.01 M_{\odot}$ and $0.072 M_{\odot}$. These assumptions, albeit simplistic, have some physical basis into what is presently known about binary systems and brown dwarfs. We outline the justification for each of them in the following.

Assumption 1. Adopting a total (stellar+substellar) system mass of $1 M_{\odot}$, the 10-2500 AU range of projected separations approximately corresponds to orbital periods of $10^{4}-10^{7.5}$ days. This range straddles the peak (at $P=10^{4.8}$ days; $a=31$ $\mathrm{AU})$ and falls along the long-period slope of the Gaussian period distribution of G-dwarf binaries (Duquennoy \& Mayor 1991). If we were to assume a similar formation scenario for 
brown dwarfs and stars, brown dwarf secondaries would also be expected to fall in frequency beyond $\sim 30$ AU separations. However, our limited amount of knowledge about brown dwarf companions suggests the opposite: brown dwarf secondaries may appear as common as stellar secondaries at $>1000 \mathrm{AU}$ separations (Gizis et al. 2001), whereas a brown dwarf desert exists at $<3 \mathrm{AU}$ semimajor axes (Marcy \& Butler 2000); Mazeh et al. 2003). A smattering of brown dwarfs has been discovered in between. A logarithmically flat distribution of semimajor axes for substellar companions, $d N / d \log a \propto a^{0}$, or equivalently $d N / d a \propto a^{-1}$, represents a middle ground between the known distribution of stellar binary orbits and the possible orbital distribution of known brown dwarf companions. The assumption is also attractive because of its conceptual and computational simplicity. As we discuss in Appendix A.4, varying the linear exponent on the semimajor axis distribution between 0 and -1 , or adopting a log-normal semimajor axis distribution as motivated by the Duquennoy \& Mayor (1991) binary period distribution, changes the overall completeness estimate by a factor of $\leqslant 1.20$.

Assumption 2. For a random distribution of orbital inclinations $i$ on the sky, the true and apparent physical separations are related by a constant multiplicative factor: the mean value of $\sin i$. However, a complication is introduced when relating the projected separation to the true semimajor axis because of the need to consider orbital eccentricity. Because an object spends a larger fraction of its orbital period near the apocenter than near the pericenter of its orbit, the ratio of the semimajor axis to the apparent separation will tend to values $>1$. Analytical treatment of the problem (Couteau 1960; van Albada 1968) shows that this happens in an eccentricity-dependent manner. Yet, when considering the eccentricity distributions of existing binary populations (Kuiper 1935a, 1935b; Duquennoy \& Mayor 1991; Fischer \& Marcy 1992), both analytical (van Albada 1968) and empirical Monte Carlo (Fischer \& Marcy 1992) approaches yield the same identical result: $\langle\log a\rangle \approx\langle\log \rho\rangle+0.1$. That is, the true semimajor axis and the measured projected separation are, on average, related by a multiplicative factor of 1.26 , such that $\langle a\rangle=1.26\langle\rho\rangle$. Given assumption 1 , this then confirms the appropriateness of the current assumption that $d N / d \log \rho \propto \rho^{0}$. Furthermore, it allows us to relate the projected separations of an ensemble of visual companions to their expected semimajor axes in a mean statistical sense.

Assumption 3. The assumption of a linearly flat substellar companion mass distribution $\left(d N / d M_{2} \propto M_{2}^{\beta} ; \beta=0\right)$ parallels results from spectroscopic studies of the IMF of isolated lowmass objects in star-forming regions (Briceño et al. 2002; Luhman et al. 2003a, 2003b; Slesnick et al. 2004; Luhman et al. 2004), which are broadly consistent with $\alpha \sim 0$ (where $\alpha$ is the exponent in $\left.d N / d M \propto M^{\alpha}\right)$. Independently, in a recent analysis of the field substellar MF, Allen et al. (4) found $\alpha=0.3 \pm 0.6$, also consistent with zero. Therefore, assuming that the substellar MFs in young stellar associations and in the field are representative of the MF of wide substellar companions, use of a linearly flat $d N / d M_{2} \propto M_{2}^{0} \mathrm{CMF}$ for our analysis is justified. This is reinforced by our subsequent $\beta=-0.39 \pm 0.36$ over the entire $0.01-1.0 M_{\odot}$ substellar and stellar companion mass range (Section 9.1.2).

The latter result may seem circuitous, since the derivation that $\beta$ is near zero is in fact dependent on the initial assumption that $\beta$ is zero. Nevertheless, we find that the initial guess for the CMF exponent is largely unimportant. As we will discuss in Appendix A.4, initial gaesses for $\beta$ ranging between -1 and 1 have a negligible effect on the final value for $\beta$.

\section{A.3. Incompleteness Analysis}

Adopting the preceding set of assumptions, we now return to the discussion of the remaining factors affecting survey incompleteness: factors (3) and (4) from Appendix A.1. We address the individual factors in three incremental steps, as pertinent to geometrical incompleteness, defined solely by the IWA and OWA of the survey and by the distribution of stellar heliocentric distances; observational incompleteness, defined by the flux limits of the survey and by the predicted brightness of substellar companions; and orbital incompleteness, defined by the fraction of orbital phase space observed. These are the same incompleteness categories as already mentioned in Section 7.1. Throughout, we adopt the aperture-normalized rms detection limits determined for each star in Section 4.3 and assume that the primary ages and distances are fixed at their mean values listed in Table 1.

\section{A.3.1. Geometrical Incompleteness}

In deciding the range of projected separations that the study is most sensitive to, we consider the full range of separations that have been explored between the IWA and OWA of the deep survey. For the IWA, we adopt 0 '.55, that is, approximately onehalf width of the $0^{\prime \prime} 1$ PALAO $K_{S}$-band PSF wider than the 0.49 radius of the PHARO coronagraph. For the OWA, we adopt 12 .'5, which is 0.3 less than the half-width of the PHARO FOV. Figure 15 shows the fraction of the deep sample stars (solid line) around which successive $1 \mathrm{AU}$ intervals are probed as a function of projected separation. It is immediately obvious that only a very narrow range of orbital separations, between $105 \mathrm{AU}$ and $125 \mathrm{AU}$, is probed around $100 \%$ of the stars. All other projected separations carry with them some degree of incompleteness that needs to be taken into account. From a purely geometrical standpoint, that is, ignoring imaging sensitivity, the limitations imposed by the choice of IWA and OWA amount to a factor of 1.96 incompleteness (for a $d N / d \log a \propto a^{0}$ semimajor axis distribution) between $6 \mathrm{AU}$ and $2375 \mathrm{AU}$ : the projected separation range contained between the IWA for the nearest star and the OWA for the farthest star in the deep sample. That is, provided that substellar companions are detectable regardless of their brightness anywhere between $0 . .55$ and $12^{\prime \prime} .5$ from each star, and provided that their distribution of semimajor axes $a$ is logarithmically flat, only about half of the companions residing in the 6-2375 AU projected separation range would be detected.

As is evident from Figure 15, such a wide range of orbital separations includes regions probed around only a small fraction of the stars. Consideration of the full 6-2375 AU range will thus induce a poorly substantiated extrapolation of the companion frequency. Instead, we choose to limit the analysis to projected separations explored around at least one-third (i.e., 33) of the stars in the deep sample. The corresponding narrower range, 22-1262 AU, is delimited by the dashed lines in Figure 15. The region has a geometrical incompleteness factor of 1.40 (compared with 1.96 for the full 6-2375 AU range above). That is, $1 / 1.40=71.4 \%$ of all companions with projected separations between 22 and $1262 \mathrm{AU}$ should be recovered in our deep survey, if they are sufficiently bright.

\section{A.3.2. Observational Incompleteness}

Following an approach analogous to that described in the preceding discussion, we infer the projected separation range over 


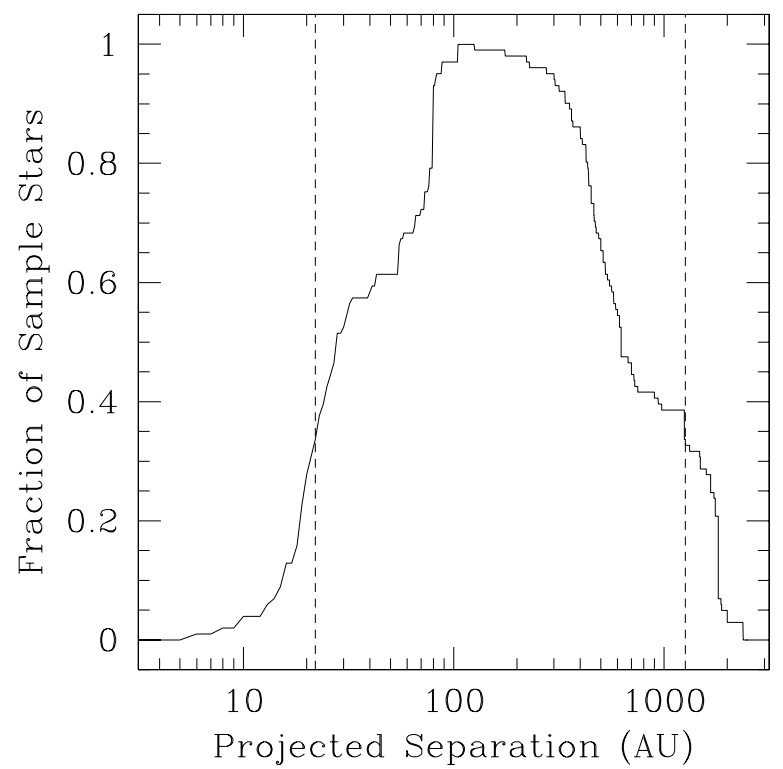

Figure 15. Projected physical separations probed in the deep sample survey. The vertical dashed lines delimit the region, 22-1262 AU, in which each $1 \mathrm{AU}$ wide projected separation interval was probed around at least one-third of the stars in our deep sample. The geometrical incompleteness factor for this region is 1.40. That is, $1 / 1.40=71.4 \%$ of all companions in the $22-1262 \mathrm{AU}$ projected separation range should have in principle been detected, had their visibility not been limited by contrast.

which our survey is sensitive to a companion of a given mass. That is, we now take into account that not all companions are sufficiently bright to be detected at all probed projected separations. Rather, their visibility is determined by their expected brightness and the attained imaging contrast.

Because mass is not an observable, we use the absolute $K$-band magnitude of a substellar object as a proxy for its mass, and employ the Lyon suite of theoretical models to convert between absolute magnitude and mass at the assumed stellar age.

We calculate the observational incompleteness of the deep survey for a grid of 11 discrete companion masses $(0.005$, $0.010,0.012,0.015,0.020,0.030,0.040,0.050,0.060,0.072$, and $0.090 M_{\odot}$ ) and over the entire 3-500 Myr age range of our deep sample. We use the DUSTY models from Chabrier et al. (2000), when the predicted companion effective temperature is above $1400 \mathrm{~K}$ (i.e., for spectral types L or earlier), and the COND models from Baraffe et al. (2003) at lower effective temperatures (spectral type T). We compare the estimated companion fluxes at the age of each of our sample stars with the corresponding flux limits for each star (see Table 6), and obtain a minimum projected separation at which a companion of a given mass would be visible around each star. Thus, summing over all stars in the deep sample, we estimate the observational incompleteness of the entire deep survey to companions of this mass.

The observational completeness estimates for each of the discrete set of $0.005-0.090 M_{\odot}$ companion masses are shown by the filled circles in Figure 16(a). The geometrical completeness limit (i.e., if companion brightness were not a limiting factor) is shown by the horizontal continuous line. Figure 16(a) demonstrates that the deep survey is nearly as complete as is theoretically possible to stellar-mass companions at angular separations between the IWA and OWA, since the observational completeness reaches the geometrical limit at $0.090 M_{\odot}$, just above the minimum hydrogen-burning mass. Figure 16(a) also illustrates that the observational completeness of the deep survey is $>50 \%$ for all substellar objects above the deuterium-burning limit. The survey completeness drops rapidly below the deuterium-burning limit because of the significantly fainter luminosities expected of nondeuterium fusing objects (e.g., Burrows et al. 2001).

With the aim to minimize our incompleteness correction, we limit our analysis to substellar companions in the $0.012-$ $0.072 M_{\odot}$ range, that is, between the deuterium- and hydrogen-
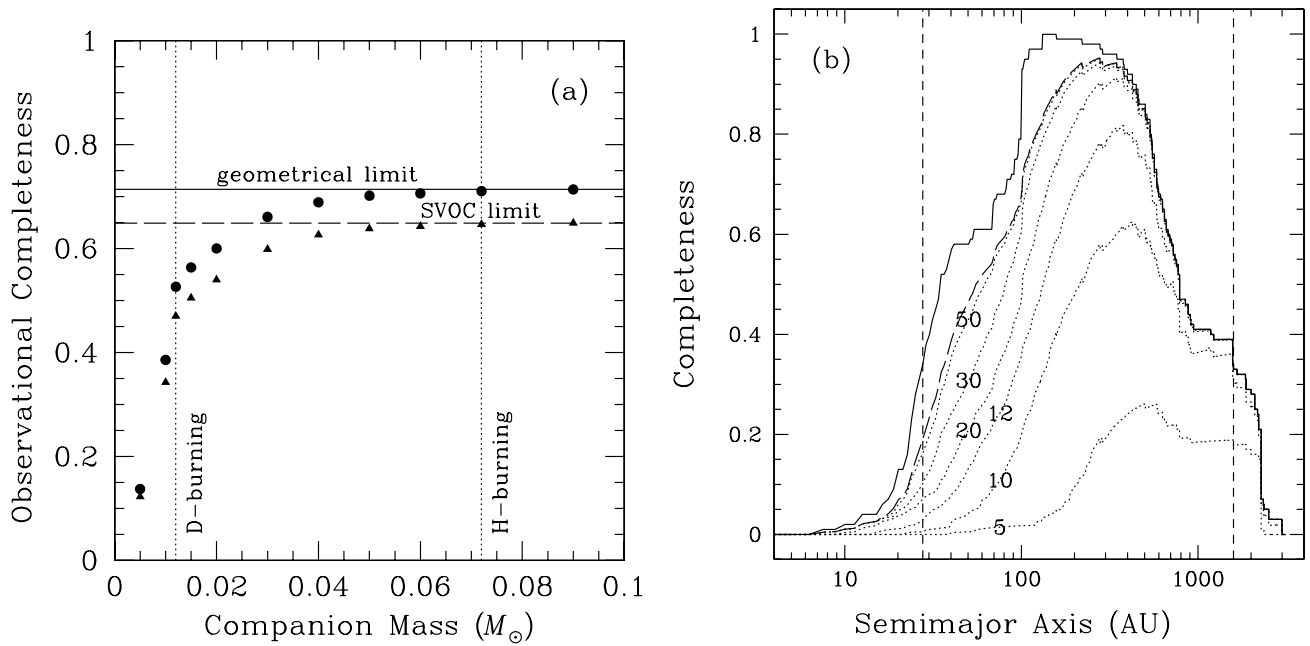

Figure 16. (a) Observational (circles) and total (triangles) completeness of the deep survey as a function of companion mass. The observational completeness at a given mass is the fraction of companions of that mass that would be detectable within a projected separation of 22-1262 AU from all sample stars (Appendix A.3.2). The total completeness is defined similarly, but for a $28-1590 \mathrm{AU}$ range of semimajor axes, and after considering the effect of varying orbital eccentricity and inclination (Appendix A.3.3). Both sets of completeness fractions are calculated assuming a logarithmically flat distribution of companion semimajor axes $a$ (Appendix A.2). The horizontal lines delimit the maximum possible observational (continuous line) and orbital (i.e., total; long-dashed line) completeness at any given mass over these AU ranges. Our definition of the orbital completeness coincides with the SVOC (see Appendix A.3.3) defined by Brown (2004). The vertical dotted lines mark the deuterium- (D) and hydrogen- (H) burning mass limits. (b) Same as Figure 15, but for the expected semimajor axes (rather than projected separations) of substellar companions and for a range of companion masses. The dotted lines are labeled with substellar masses in units of $M_{\odot} / 1000$. The solid curve delineates the geometrical completeness limit and the long-dashed curve the SVOC limit (see panel a). The scale of the abcissa has been adjusted with respect to that in Figure 15 to reflect the 1.26 multiplicative factor in the switch from projected separation to semimajor axis. 
burning mass limits. Depending on the assumed functional form of the CMF, the sum of the geometrical + observational completeness in this mass range is between $53.0 \%$ and $71.3 \%$. Adopting a $d N / d M_{2} \propto M_{2}^{0}$ (i.e., $\beta=0$ ) CMF (Appendix A.2), we find that the survey is $68.2 \%$ complete to $0.012-0.072 M_{\odot}$ substellar companions at projected separations of 22-1262 AU from their host stars.

\section{A.3.3. Orbital Incompleteness}

The analysis so far has dealt only with the projected separation of substellar companions. We now consider the effect of realistic orbital semimajor axes, inclinations, and eccentricities.

We first adopt the multiplicative factor of 1.26 to relate the projected separation $\rho$ to the true semimajor axis $a:\langle a\rangle=$ $1.26\langle\rho\rangle$ (see Appendix A.2). That is, the orbital semimajor axes probed by the survey are on average a factor of 1.26 larger, 28-1590 AU, than the range of probed projected separations.

The multiplicative transformation from $\langle\rho\rangle$ to $\langle a\rangle$ does not exhaust the discussion of orbital incompleteness. Because companions on orbits with nonzero inclinations and eccentricities spend most of their time at projected separations $\rho \neq a / 1.26$, they may still be missed in the survey. The most likely scenarios in which this can occur are for companions on highly inclined and/or eccentric orbits.

With a small number of positive substellar companion detections, orbital incompleteness issues are best addressed through Monte Carlo simulations. Such has been performed for a wide range of realistic orbital inclinations and eccentricities in a study by Brown (2004), the results of which we adopt here. Brown's work investigated the detectability of populations of habitable extrasolar terrestrial planets with a range of orbital distributions by the Terrestrial Planet Finder-Coronagraph $(T P F-C)$. Although the angular scales and the levels of imaging contrast between the present coronagraphic survey and the design specifications for $T P F-C$ are vastly different $(T P F-C$ projections call for a factor of $\approx 2.5$ smaller IWA and $\sim 10^{6}$ higher contrast), the problem is conceptually the same: to determine the completeness to orbits with a certain semimajor axis, given an opaque coronagraph of a fixed radius. Brown (2004) parameterized this problem in terms of the ratio $\zeta$ (which he defined as $\alpha$ ) of the semimajor axis to the obscuration radius, so his results are universally scalable. His analysis did not include treatment of imaging contrast or limiting flux (these are addressed in a follow-up work: 14), which makes it suitable to apply to results that have already been corrected for these effects, as we have already done for our survey in Appendix A.3.2.

Brown (2004) found that the detectability of orbiting companions in a single-visit observation, what he terms the "single visit obscurational completeness" (SVOC), is a strong function of $\zeta$ between $\zeta=1$ and 2 . The SVOC varies between $\approx 30 \%$ at $\zeta=1$ and $\approx 85 \%$ at $\zeta=1.9$ (Figure 3 in Brown 2004). Higher SVOC, at the $95 \%$ and $99 \%$ levels, is achieved only for $\zeta=3.2$ and 7.1 , respectively, that is, far from the coronagraphic edge. The result is largely independent $(<10 \%$ variation $)$ of the assumed orbital eccentricity $e$ for $0 \leqslant e \leqslant 0.35$.

We adopt the results of Brown's analysis and use the SVOC values for a representative orbital eccentricity of 0.35 (Table 4 in Brown 2004) - a value near the peak of the eccentricity distribution of G-dwarf binaries with $>10^{3}$ day periods (Duquennoy \& Mayor 1991). We calculate the SVOC on the deepest image of each sample star, for each of the discrete candidate companion masses in the $0.005-0.090 M_{\odot}$ range considered in Appendix A.3.2. We define the minimum projected separation at which a companion of a given mass is detectable as the effective obscuration radius for that companion mass. The results from the combined treatment of the observational completeness (Appendix A.3.2) and the SVOC are shown in Figure 16(a) by filled triangles and in Figure 16(b) with the dotted lines. The longdashed lines in Figures 16(a) and 16(b) delimit the maximum attainable SVOC, that is, when the companion brightness is not a limiting factor. Figure 16(a) shows that the completeness to $\geqslant$ $0.072 M_{\odot}$ objects is very near $(64.8 \%)$ the SVOC limit $(64.9 \%)$. That is, the deep survey is almost maximally complete to stellar companions. The survey is only $47.0 \%$ complete to companions at the low end of the brown dwarf mass range at $0.012 M_{\odot}$.

The additional consideration of orbital incompleteness does not significantly affect the overall incompleteness of the survey within the posited 22-1262 AU projected separation (281590 AU semimajor axis) range. Given the assumed companion mass and orbital semimajor axis distributions (Appendix A.2), the overall (geometrical + observational + orbital) completeness becomes $62.0 \%$.

We note that the consideration of the SVOC, as defined by Brown (2004), does not address all possibilities for orbital incompleteness. Other than being obscured by the coronagraph or lost in the glare of its host star, a companion on a highly eccentric orbit may fall outside the OWA, even if its semimajor axis was in the explored range. This additional factor is not taken into account here. However, judging by the small decrease $(68.2 \%-62.0 \%=6.2 \%)$ in the overall incompleteness correction induced by the consideration of the SVOC, it is unlikely that inclusion of the remaining factors affecting orbital incompleteness will decrease the overall survey completeness below $50 \%$.

\section{A.4. Effect of Variations in the Assumed Companion Semimajor Axis and Period Distributions}

The above final completeness estimate is based on the assumptions for the semimajor axis and companion mass distributions adopted in Appendix A.2. These assumptions are merely guesses, and in reality the companion orbital and mass distributions may take different forms. Indeed, in Section 9.2, we argued that the orbital period distributions of substellar and stellar mass companions are probably the same, while in Section 9.1.2, we concluded that the MFs of companions and isolated objects are different. Both of these results are at odds with the corresponding assumptions. It is conceivable that other initial guesses for the orbital and mass distributions of the companions may lead to different conclusions.

We therefore analyzed the completeness of the survey to substellar companions under a broader set of functional forms for the companion semimajor axis and mass distributions. For the semimajor axis distribution, we also considered (1) the equivalent of the log-normal orbital period distribution for Sunlike binary stars from Duquennoy \& Mayor (1991) under the assumption that the total system mass is $1 M_{\odot} ;(2)$ the extrasolar planet period $d N / d \log P \propto P^{0.26}$ distribution from Cumming et al. (2008), which converts to $d N / d a \propto a^{-0.61}$ for solar-mass primaries; and (3) a linearly flat $d N / d a \propto a^{0}$ distribution. For the $\mathrm{CMF}$ exponent $\beta$, we tested values in the -1 to 1 range.

The estimates for the completeness to substellar companions for the three different semimajor axis distributions with $\beta$ fixed at zero were $62.7 \%, 59.9 \%$, and $51.9 \%$, respectively, all within a factor of 1.20 of the one already obtained in Appendix A.3.3. In particular, we note that the assumption of either the star- 
like log-normal or the planet-like period distribution altered the completeness estimate very little (by a factor of $\leqslant 1.04$ ). If we set the CMF index $\beta$ to either -1 or 1 but held the assumed semimajor axis distribution fixed at $d N / d a \propto a^{-1}$, the completeness became $58.1 \%$ or $64.9 \%$, respectively. If we allowed both of the companion orbital and mass distributions to vary, the completeness estimates ranged from $50.2 \%$ to $66.0 \%$.

Overall, we found that the inferred frequency of wide substellar companions to young solar analogs in Section 8.1 would be affected by a factor of $\leqslant 1.24$. In the likely case that the orbital period distributions of substellar and stellar companions are the same, as in Duquennoy \& Mayor (1991), our inferred frequency would be accurate to a within a factor of 1.06 .

Such small changes to the incompleteness estimate of our survey affect the resultant CMF power-law index $\beta$ only minimally. Because the relative changes in the completenesscorrected numbers per mass bin of the CMF are much smaller than the bin to bin variations, the effect on the fitted value for $\beta$ is well within the derived $1 \sigma$ range.

\section{A.5. Summary of Incompleteness Analysis and Further Considerations}

We adopt $62 \%$ as the final estimate for the completeness to substellar companions in our deep survey. That is, given two detected brown dwarf companions with semimajor axes in the $28-1590 \mathrm{AU}$ range, on average $0.62^{-1}=1.6$ more companions with semimajor axes in the same range have been missed. This estimate is based on the combined consideration of the geometrical, observational, and orbital incompleteness factors described in Appendix A.3.

In closing, we recall that because the physical association status of a large fraction $(30.3 \%)$ of candidate companions discovered in the survey remains undecided (Section 5.2), it is possible that more bona fide substellar companions may be confirmed in this data set in the future. This is not very likely, given that the vast majority of the undecided candidates are faint, reside in relatively high-density fields, and are at wide angular separations from their candidate primaries (Figure 6), that is, they have very high probabilities of being background stars. Because of the presently unknown and likely unimportant nature of the additional candidate companions, and for the sake of preserving statistical rigor, we have assumed that none of the remaining candidates are bona fide brown dwarfs and that the derived value of $62 \%$ provides an accurate estimate of the completeness of our deep survey.

\section{REFERENCES}

Adams, J. D., Stauffer, J. R., Monet, D. G., Skrutskie, M. F., \& Beichman, C. A. 2001, AJ, 121, 2053

Ali, B., Carr, J. S., Depoy, D. L., Frogel, J. A., \& Sellgren, K. 1995, AJ, 110, 2415

Allen, P. R. 2007, ApJ, 668, 492

Allen, P. R., Koerner, D. W., Reid, I. N., \& Trilling, D. E. 2005, ApJ, 625, 385

Andersen, M., Meyer, M. R, Greissl, J., \& Aversa, A. 2008, ApJ, 683, 183

Baraffe, I., Chabrier, G., Allard, F., \& Hauschildt, P. H. 1998, A\&A, 337, 403

Baraffe, I., Chabrier, G., Barman, T. S., Allard, F., \& Hauschildt, P. H. 2003, A\&A, 402, 701

Barrado y Navascues, D., Fernandez-Figueroa, M. J., Garcia Lopez, R. J., de Castro, E., \& Cornide, M. 1997, A\&A, 326, 780

Bate, M. R., \& Bonnell, I. A. 2005, MNRAS, 356, 1201

Bate, M. R., Bonnell, I. A., \& Bromm, V. 2002, MNRAS, 332, L65
Biller, B. A., et al. 2007, ApJS, 173, 143

Bloemhof, E. E. 2003, ApJ, 582, L59

Boccaletti, A., Riaud, P., \& Rouan, D. 2002, PASP, 114, 132

Bouvier, J., Rigaut, F., \& Nadeau, D. 1997, A\&A, 323, 139

Brandner, W., et al. 2000, AJ, 120, 950

Briceño, C., Luhman, K. L., Hartmann, L., Stauffer, J. R., \& Kirkpatrick, J. D. 2002, ApJ, 580, 317

Brown, R. A. 2004, ApJ, 607, 1003

Brown, R. A. 2005, ApJ, 624, 1010

Burgasser, A. J., Kirkpatrick, J. D., \& Lowrance, P. J. 2005, AJ, 129, 2849

Burgasser, A. J., Reid, I. N., Siegler, N., Close, L., Allen, P., Lowrance, P., \& Gizis, J. 2007, in Protostars and Planets V, ed. B. Reipurth, D. Jewitt, \& K. Keil (Tuscon, AZ: Univ. Arizona Press), 427

Burrows, A., Hubbard, W. B., Lunine, J. I., \& Liebert, J. 2001, Rev. Mod. Phys., 73, 719

Burrows, A., et al. 1997, ApJ,, 491, 856

Cameron, P. B., Britton, M. C., \& Kulkarni, S. R. 2009, AJ, 137, 83

Cannon, A. J., \& Pickering, E. C. 1918, Ann. Harvard College Obs., 91

Carpenter, J. M., et al. 2008, ApJS, 179, 324

Carson, J. C., Eikenberry, S. S., Brandl, B. R., Wilson, J. C., \& Hayward, T. L. 2005, AJ, 130, 1212

Casagrande, L., Flynn, C., \& Bessell, M. 2008, MNRAS, 389, 585

Chabrier, G. 2001, ApJ, 554, 1274

Chabrier, G. 2003, PASP, 115, 763

Chabrier, G. 2005, The Initial Mass Function: from Salpeter 1955 to 2005 , in The Initial Mass Function 50 years later, eds. E. Corbelli, F. Palle, \& H. Zinnecker, Astrophysics and Space Science Library vol. 327, (Dordrecht: Springer), p. 41

Chabrier, G., Baraffe, I., Allard, F., \& Hauschildt, P. 2000, ApJ, 542, 464

Chauvin, G., Lagrange, A.-M., Dumas, C., Zuckerman, B., Mouillet, D., Song, I., Beuzit, J.-L., \& Lowrance, P. 2005a, A\&A, 438, L25

Chauvin, G., et al. 2005b, A\&A, 438, L29

Couteau, P. 1960, J. Obs., 43, 41

Cox, A. N. 2000, in Allen's Astrophysical Quantities, ed. A. N. Cox (4th ed.; New York: Springer), p. 389

Cumming, A., Butler, R. P., Marcy, G. W., Vogt, S. S., Wright, J. T., \& Fischer, D. A. 2008, PASP, 120,531

Cushing, M. C., Rayner, J. T., \& Vacca, W. D. 2005, ApJ, 623, 1115

Cutri, R. M., et al. 2003, 2MASS All Sky Catalog of Point Sources (The IRSA 2MASS All-Sky Point Source Catalog, (NASA/IPAC Infrared Science Archive http://irsa.ipac.caltech.edu/applications/Gator/)

Dahn, C. C., et al. 2002, AJ, 124, 1170

D’Antona, F., \& Mazzitelli, I. 1994, ApJS, 90, 467

de Zeeuw, P. T., Hoogerwerf, R., de Bruijne, J. H. J., Brown, A. G. A., \& Blaauw, A. 1999, AJ, 117, 354

Diolaiti, E., Bendinelli, O., Bonaccini, D., Close, L., Currie, D., \& Parmeggiani, G. 2000, A\&AS, 147, 335

Duquennoy, A., \& Mayor, M. 1990, in Duplicity of Solar-Like Stars in the Solar Neighbourhood, in New Windows to the Universe Proc. XIth European of the IAU, ed. F. Sanchez \& M. Vazqunes (Cambridge: Cambridge Univ. Press), 253

Duquennoy, A., \& Mayor, M. 1991, A\&A, 248, 485

Fabricius, C., \& Makarov, V. V. 2000, A\&A, 356, 141

Fischer, D. A., \& Marcy, G. W. 1992, ApJ, 396, 178

Fischer, D. A., Marcy, G. W., Butler, R. P., Vogt, S. S., Walp, B., \& Apps, K. 2002, PASP, 114, 529

Freeman, P. E., Kashyap, V., Rosner, R., \& Lamb, D. Q. 2002, ApJS, 138, 185

Gaidos, E. J., Henry, G. W., \& Henry, S. M. 2000, AJ, 120, 1006

Ghez, A. M., McCarthy, D. W., Patience, J. L., \& Beck, T. L. 1997, ApJ, 481, 378

Ghez, A. M., Neugebauer, G., \& Matthews, K. 1993, AJ, 106, 2005

Gizis, J. E., Kirkpatrick, J. D., Burgasser, A., Reid, I. N., Monet, D. G., Liebert, J., \& Wilson, J. C. 2001, ApJ, 551, L163

Goldin, A., \& Makarov, V. V. 2006, ApJS, 166, 341

Goldin, A., \& Makarov, V. V. 2007, ApJS, 173, 137

Golimowski, D. A., et al. 2004, AJ, 127, 3516

Gorlova, N. I., Meyer, M. R., Rieke, G. H., \& Liebert, J. 2003, ApJ, 593, 1074

Goto, M., et al. 2003, in Proc. SPIE 4839, Adaptive Optical Systems Technologies II, ed. P. L. Wizinowich \& D. Bonaccini (Bellingham, WA: SPIE), $1117-23$

Guenther, E. W., Esposito, M., Mundt, R., Covino, E., Alcalá, J. M., Cusano, F., \& Stecklum, B. 2007, A\&A, 467, 1147

Gutiérrez, C. M., García López, R. J., Rebolo, R., Martín, E. L., \& François, P. 1999, A\&AS, 137, 93

Halbwachs, J. L., Mayor, M., Udry, S., \& Arenou, F. 2003, A\&A, 397, 159 
Hale, A. 1994, AJ, 107, 306

Hartkopf, W. I., \& Mason, B. D. 2003 (Washington, DC: US Naval Observatory http://ad.usno.navy.mil/wds/orb6/orb6c.html

Hartkopf, W. I., Mason, B. D., \& McAlister, H. A. 1996, AJ, 111, 370

Hartkopf, W. I., Mason, B. D., \& Worley, C. E. 2001, AJ, 122, 3472

Hayward, T. L., Brandl, B., Pirger, B., Blacken, C., Gull, G. E., Schoenwald, J., \& Houck, J. R. 2001, PASP, 113, 105

Heckmann, O., Dieckvoss, W., \& Kox, H. 1956, Astron. Nachr., 283, 109

Hertzspring, E. 1947, Annalen Sterrewacht Leiden, 19, 1

Hillenbrand, L. A., \& Carpenter, J. M. 2000, ApJ, 540, 236

Hillenbrand, L. A., \& White, R. J. 2004, ApJ, 604, 741

Hinz, J. L., McCarthy, D. W., Jr., Simons, D. A., Henry, T. J., Kirkpatrick, J. D., \& McGuire, P. C. 2002, AJ, 123, 2027

Høg, E., et al. 2000, A\&A, 355, L27

Jayawardhana, R., \& Brandeker, A. 2001, ApJ, 561, L111

Jefferies, S. M., \& Christou, J. C. 1993, ApJ, 415, 862

Kastner, J. H., Zuckerman, B., Weintraub, D. A., \& Forveille, T. 1997, Science, 277,67

Kirkpatrick, J. D., Dahn, C. C., Monet, D. G., Reid, I. N., Gizis, J. E., Liebert, J., \& Burgasser, A. J. 2001, AJ, 121, 3235

Köhler, R., Kunkel, M., Leinert, C., \& Zinnecker, H. 2000, A\&A, 356, 541

Kohler, R., \& Leinert, C. 1998, A\&A, 331, 977

König, B., Guenther, E. W., Woitas, J., \& Hatzes, A. P. 2005, A\&A, 435, 215

Kouwenhoven, M. B. N., Brown, A. G. A, Goodwin, S. P., Portegies Zwart, S. F., \& Kaper, L. 2008, A\&A, in press (arXiv:0811.2859)

Kouwenhoven, M. B. N., Brown, A. G. A., \& Kaper, L. 2007, A\&A, 464, 581

Kouwenhoven, M. B. N., Brown, A. G. A., Zinnecker, H., Kaper, L., \& Portegies Zwart, S. F. 2005, A\&A, 430, 137

Kraft, R. P., Burrows, D. N., \& Nousek, J. A. 1991, ApJ, 374, 344

Kraus, A. L., \& Hillenbrand, L. A. 2007, ApJ, 664, 1167

Kraus, A. L., Ireland, M. J., Martinache, F., \& Lloyd, J. P. 2008, ApJ, 679, 762

Kroupa, P. 2001, MNRAS, 322, 231

Kroupa, P. 2002, Science, 295, 82

Kroupa, P., Tout, C. A., \& Gilmore, G. 1990, MNRAS, 244, 76

Kuiper, G. P. 1935a, PASP, 47, 15

Kuiper, G. P. 1935b, PASP, 47, 121

Lafrenière, D., et al. 2007, ApJ, 670, 1367

Lafrenière, D., Jayawardhana, R., Brandeker, A., Ahmic, M., \& van Kerkwijk, M. H. 2008, ApJ, 683, 844

Leggett, S. K., Allard, F., Berriman, G., Dahn, C. C., \& Hauschildt, P. H. 1996, ApJS, 104, 117

Leggett, S. K., et al. 2002, ApJ, 564, 452

Liu, M. C., Dupuy, T. J., \& Ireland, M. J. 2008, ApJ, 689, 436

Liu, M. C., Fischer, D. A., Graham, J. R., Lloyd, J. P., Marcy, G. W., \& Butler, R. P. 2002, ApJ, 571, 519

Lowrance, P. J., et al. 2005, AJ, 130, 1845

Luhman, K. L. 1999, ApJ, 525, 466

Luhman, K. L. 2004, ApJ, 617, 1216

Luhman, K. L., Briceño, C., Stauffer, J. R., Hartmann, L., Barrado, y, Navascués, D., \& Caldwell, N. 2003a, ApJ, 590, 348

Luhman, K. L., \& Jayawardhana, R. 2002, ApJ, 566, 1132

Luhman, K. L., Joergens, V., Lada, C., Muzerolle, J., Pascucci, I., \& White, R. 2007a, in Protostars and Planets V, ed. B. Reipurth, D. Jewitt, \& K. Keil (Tuscon, AZ: Univ. Arizona Press), 443

Luhman, K. L., McLeod, K. K., \& Goldenson, N. 2005, ApJ, 623, 1141

Luhman, K. L., Peterson, D. E., \& Megeath, S. T. 2004, ApJ, 617, 565

Luhman, K. L., Stauffer, J. R., Muench, A. A., Rieke, G. H., Lada, E. A., Bouvier, J., \& Lada, C. J. 2003b, ApJ, 593, 1093

Luhman, K. L., et al. 2007b, ApJ, 654, 570

Makarov, V. V., \& Kaplan, G. H. 2005, AJ, 129, 2420

Mamajek, E. E. 2004, PhD thesis, Univ. of Arizona

Mamajek, E. E., \& Hillenbrand, L. A. 2008, ApJ, 687, 1264

Mamajek, E. E., Lawson, W. A., \& Feigelson, E. D. 1999, ApJ, 516, L77

Mamajek, E. E., Meyer, M. R., \& Liebert, J. 2002, AJ, 124, 1670

Marcy, G. W., \& Butler, R. P. 2000, PASP, 112, 137

Marois, C., Lafrenière, D., Doyon, R., Macintosh, B., \& Nadeau, D. 2006, ApJ, 641,556

Marois, C., Lafrenière, D., Macintosh, B., \& Doyon, R. 2008, ApJ, 673, 647

Masciadri, E., Mundt, R., Henning, T., Alvarez, C., \& Navascués Barrado y, D. 2005, ApJ, 625, 1004

Mason, B. D., Wycoff, G. L., Hartkopf, W. I., Douglass, G. G., \& Worley, C. E. 2001, AJ, 122, 3466

Mazeh, T., Simon, M., Prato, L., Markus, B., \& Zucker, S. 2003, ApJ, 599, 1344

McCarthy, C., \& Zuckerman, B. 2004, AJ, 127, 2871

McElwain, M. W., et al. 2007, ApJ, 656, 505

Mermilliod, J.-C., Rosvick, J. M., Duquennoy, A., \& Mayor, M. 1992, A\&A, 265,513
Metchev, S. A. 2006, PhD thesis, California Institute of Technology

Metchev, S. A., \& Hillenbrand, L. A. 2004, ApJ, 617, 1330

Metchev, S. A., \& Hillenbrand, L. A. 2006, ApJ, 651, 1166

Metchev, S. A., Hillenbrand, L. A., \& White, R. J. 2003, ApJ, 582, 1102

Metchev, S. A., Kirkpatrick, J. D., Berriman, G. B., \& Looper, D. 2008, ApJ, 676, 1281

Meyer, M. R., et al. 2006, PASP, 118, 1690

Mohanty, S., Jayawardhana, R., Huélamo, N., \& Mamajek, E. 2007, ApJ, 657, 1064

Montes, D., López-Santiago, J., Gálvez, M. C., Fernández-Figueroa, M. J., De Castro, E., \& Cornide, M. 2001, MNRAS, 328, 45

Mugrauer, M., et al. 2004, A\&A, 417, 1031

Munari, U., Dallaporta, S., Siviero, A., Soubiran, C., Fiorucci, M., \& Girard, P. 2004, A\&A, 418, L31

Neuhäuser, R., \& Guenther, E. W. 2004, A\&A, 420, 647

Nordström, B., et al. 2004, A\&A, 418, 989

Oppenheimer, B. R., Dekany, R. G., Hayward, T. L., Brandl, B., Troy, M., \& Bloemhof, E. E. 2000, in Proc. SPIE 4007, Adaptive Optical Systems Technology, ed. P. L. Wizinowich (Bellingham, WA: SPIE), 899-905

Oppenheimer, B. R., Golimowski, D. A., Kulkarni, S. R., Matthews, K. Nakajima, T., Creech-Eakman, M., \& Durrance, S. T. 2001, AJ, 121, 2189

Pan, X., Shao, M., \& Kulkarni, S. R. 2004, Nature, 427, 326

Papoulis, A. 1984, Probability, Random Variables, and Stochastic Processes (New York: McGraw-Hill)

Patel, S. G., Vogt, S. S., Marcy, G. W., Johnson, J. A., Fischer, D. A., Wright, J T., \& Butler, R. P. 2007, ApJ, 665, 744

Patience, J., Ghez, A. M., Reid, I. N., \& Matthews, K. 2002, AJ, 123, 1570

Patience, J., Ghez, A. M., Reid, I. N., Weinberger, A. J., \& Matthews, K. 1998, AJ, 115, 1972

Perryman, M. A. C., et al. 1997, A\&A, 323, L49

Perryman, M. A. C., et al. 1998, A\&A, 331, 81

Potter, D., Martín, E. L., Cushing, M. C., Baudoz, P., Brandner, W., Guyon, O., \& Neuhäuser, R. 2002, ApJ, 567, L133

Pounds, K. A., et al. 1993, MNRAS, 260, 77

Pourbaix, D. 2000, A\&AS, 145, 215

Preibisch, T., Brown, A. G. A., Bridges, T., Guenther, E., \& Zinnecker, H 2002, AJ, 124, 404

Preibisch, T., \& Zinnecker, H. 1999, AJ, 117, 2381

Pye, J. P., et al. 1995, MNRAS, 274, 1165

Rainwater, L. J., \& Wu, C. S. 1947, Nucleonics, 1, 60

Reid, I. N., \& Hawley, S. L. 2005, New Light on Dark Stars Red Dwarfs, Low-Mass Stars, Brown Stars, Springer-Praxis Books in Astrophysics and Astronomy, ed. I. N. Reid \& S. L. Hawley (Chichester: Praxis Publishing Ltd), p. 169

Reid, I. N., et al. 2004, AJ, 128, 463

Scardia, M. 1979, Astron. Nachr., 300, 307

Schroeder, D. J., et al. 2000, AJ, 119, 906

Shatsky, N., \& Tokovinin, A. 2002, A\&A, 382, 92

Sivaramakrishnan, A., Koresko, C. D., Makidon, R. B., Berkefeld, T., \& Kuchner, M. J. 2001, ApJ, 552, 397

Skrutskie, M. F., et al. 2006, AJ, 131, 1163

Slesnick, C. L., Hillenbrand, L. A., \& Carpenter, J. M. 2004, ApJ, 610, 1045

Söderhjelm, S. 1999, A\&A, 341, 121

Southworth, J., Maxted, P. F. L., \& Smalley, B. 2005, A\&A, 429, 645

Sterzik, M. F., \& Durisen, R. H. 2004, RevMexA\&A, 21, 58

Stetson, P. B. 1992, in ASP Conf. Ser. 25, Astronomical Data Analysis Software and Systems I, (San Francisco, CA: ASP), 297

Strassmeier, K., Washuettl, A., Granzer, T., Scheck, M., \& Weber, M. 2000, A\&AS, 142, 275

Swenson, F. J., Faulkner, J., Rogers, F. J., \& Iglesias, C. A. 1994, ApJ, 425, 286

Tanner, A., et al. 2007, PASP, 119, 747

Thompson, D., Egami, E., \& Sawicki, M. 2001, The Keck Near Infrared AO Camera. Pre-ship testing., California Institute of Technology http://www2.keck.hawaii.edu/inst/nirc2/preship_testing.pdf

Tinney, C. G., Mould, J. R., \& Reid, I. N. 1993, AJ, 105, 1045

Tokovinin, A., Thomas, S., Sterzik, M., \& Udry, S. 2006, A\&A, 450, 681

Tokovinin, A. A., Chalabaev, A., Shatsky, N. I., \& Beuzit, J. L. 1999, A\&A, 346,481

Troy, M., et al. 2000, in Proc. SPIE 4007, Adaptive Optical Systems Technology, ed. P. L. Wizinowich (Bellingham, WA: SPIE), 31

van Albada, T. S. 1968, Bull. Astron. Inst. Neth., 20, 47

van Bueren, H. G. 1952, Bull. Astron. Inst. Neth., 11, 385

van Leeuwen, F. 1999, A\&A, 341, L71

van Leeuwen, F., Alphenaar, P., \& Brand, J. 1986, A\&AS, 65, 309

Ventura, P., Zeppieri, A., Mazzitelli, I., \& D’Antona, F. 1998, A\&A, 334,953 
Voges, W., et al. 1999, A\&A, 349, 389

Voges, W., et al. 2000, IAU Circ., 7432, 1

Vrba, F. J., et al. 2004, AJ, 127, 2948

Walter, F. M., Vrba, F. J., Mathieu, R. D., Brown, A., \& Myers, P. C. 1994, AJ, 107,692

Whipple, F. L. 1966, Smithsonian Astrophysical Observatory Star Catalog (Smithsonian Astrophysical Observatory Star Catalog, Washington: Smithsonian Institution Press)

White, R. J., Gabor, J. M., \& Hillenbrand, L. A. 2007, AJ, 133, 2524

Wichmann, R., Schmitt, J. H. M. M., \& Hubrig, S. 2003, A\&A, 399, 983
Wilson, J. C., Kirkpatrick, J. D., Gizis, J. E., Skrutskie, M. F., Monet, D. G., \& Houck, J. R. 2001, AJ, 122, 1989

Wizinowich, P., et al. 2000, PASP, 112, 315

Zacharias, N., Urban, S. E., Zacharias, M. I., Wycoff, G. L., Hall, D. M., Monet, D. G., \& Rafferty, T. J. 2004, AJ, 127, 3043

Zuckerman, B., \& Song, I. 2004, ApJ, 603, 738

Zuckerman, B., \& Song, I. 2008, A\&A, in press (arXiv:0811.0429)

Zuckerman, B., Song, I., Bessell, M. S., \& Webb, R. A. 2001, ApJ, 562, L87

Zuckerman, B., \& Webb, R. A. 2000, ApJ, 535, 959

Zwahlen, N., North, P., Debernardi, Y., Eyer, L., Galland, F., Groenewegen, M. A. T., \& Hummel, C. A. 2004, A\&A, 425, L45 